Fall 12-18-2020

\title{
Mechanisms by Which MnTE-2-PyP Suppresses Prostate Cancer Cell Growth
}

\author{
Yuxiang Zhu \\ University of Nebraska Medical Center
}

Tell us how you used this information in this short survey.

Follow this and additional works at: https://digitalcommons.unmc.edu/etd

Part of the Biochemistry Commons, Biology Commons, Cancer Biology Commons, Cell Biology Commons, Chemicals and Drugs Commons, Immunotherapy Commons, and the Molecular Biology Commons

\section{Recommended Citation}

Zhu, Yuxiang, "Mechanisms by Which MnTE-2-PyP Suppresses Prostate Cancer Cell Growth" (2020). Theses \& Dissertations. 501.

https://digitalcommons.unmc.edu/etd/501

This Dissertation is brought to you for free and open access by the Graduate Studies at DigitalCommons@UNMC. It has been accepted for inclusion in Theses \& Dissertations by an authorized administrator of DigitalCommons@UNMC.For more information, please contact digitalcommons@unmc.edu. 


\title{
MECHANISMS BY WHICH MNTE-2-PYP SUPPRESSES PROSTATE CANCER CELL GROWTH
}

\author{
by \\ Yuxiang Zhu \\ A DISSERTATION \\ Presented to the Faculty of \\ the University of Nebraska Graduate College \\ in Partial Fulfillment of the Requirements \\ for the Degree of Doctor of Philosophy \\ Biochemistry \& Molecular Biology \\ Graduate Program \\ Under the Supervision of Professor Rebecca Oberley-Deegan
University of Nebraska Medical Center
Omaha, NE \\ December, 2020 \\ Supervisory Committee: \\ Datta, Kaustubh, Ph.D. \\ Zimmerman, Matthew, Ph.D. \\ Teoh-Fitzgerald, Melissa, Ph.D.
}




\section{ACKNOWLEDGEMENTS}

I would like to express my sincere gratitude to the following people who have helped me during the last 5 years. Without them, it would have been impossible for me to complete my research.

First of all, I wanted to thank my mentor, Dr. Rebecca Oberley-Deegan, for providing me research opportunities, academic guidence, life and spiritual support throughout the entire Ph.D. program. Her patience on my training is the biggest power that encouraged and inspired me from the beginning and from now on. For every presentation, every grant application, and every paper I have ever worked on, she spent a lot of time revising them and gave me thorough advice. Without her, I might have failed. The black and white characters cannot fully express my gratitude to my mentor, but beyond the paper, my future success in academic area may work as a proof.

I would like to thank my current committee members, Dr. Melissa Teoh-Fitzgerald, Dr. Kaustubh Datta, and Dr. Matthew Zimmerman, for their knowledge and advice that supported my projects. I really appreciate the valuable opportunities of teaching experiences kindly provided by Dr.Teoh-Fitzgerald. Her knowledge on redox biology also addressed the difficulties I encountered in my project during the last years. The advice from Dr. Datta on immunology project was critical and visionary. Without him, I may not have enough time to discover the central mechanisms of our compounds. From Dr. Zimmerman, I still remember the first time we met and talked about the ROS-Glo kit during the EPR experiment, from which I learned what should be critical thinking and late on, this deeply changed my understanding of how to evaluate an assay properly. Additionally, I also wanted to thank Dr. Kate Hyde as the chairman of my comprehensive exam committee who provided valuable suggestions and guidance for my comprehensive exam. 
I have worked with Dr. Elizabeth Kosmacek and Dr. Arpita Chatterjee day by day for 5 years, which is not a short time for human beings. To me, they are like elder sisters and they treat me like brother. They have taught me so many technologies, helped me on my experiments, and solved my problems. Their professionalism and diligence on science serve as great models for me. Beyond these, they help me in daily life and especially during the covid19 hard time. As an ancient Chinese saying goes, their support is the burning fire in a snowstorm.

I also wanted to address my thanks to my previous and current graduate student lab members. I would like to thank Dr. Shashank Shrishrimal who worked with me on several important experiments and also gave me help in the social life. I would like to thank Eliezer Lichter for helping me fix the car and teaching me get around daily problems. And from Joshua McDowell, I learned a different view of science and society, and he is also kind-hearted and helpful in my research.

Specifically, I would like to thank Karen Hankins. She is the graduate student education program associate of our department and she always saved me in time when I faced administrative troubles.

Finally, I would like to thank my parents, relatives, and friends during this journey, especially my father, Jianrong Zhu, my mother, Haiping $\mathrm{Xu}$, and my uncle, Hua Tang. It was their financial and spiritual support that realized my dream studying in this country. Although the Pacific Ocean is between us, I can always gain strength from the other side. The journey has not ended yet, and the past time shall long stay in my heart. 


\section{ABSTRACT}

\section{MECHANISMS BY WHICH MNTE-2-PYP SUPPRESSES PROSTATE CANCER CELL GROWTH}

Prostate cancer patients are often treated with radiotherapy. MnTE-2-PyP, is a superoxide dismutase (SOD) mimic and a known radioprotector of normal tissues. Our recent work demonstrates that MnTE-2-PyP also inhibits prostate cancer progression with radiotherapy; however, the mechanisms remain unclear. In this thesis, we identified that MnTE-2-PyP-induced intracellular $\mathrm{H}_{2} \mathrm{O}_{2}$ levels are critical in inhibiting growth of prostate cancer cells. We found that MnTE-2-PyP induced protein oxidations in PC3 cells and one major group of oxidized protein targets were involved in energy metabolism. The oxidative phosphorylation rates were significantly enhanced in both PC3 and LNCaP cells with MnTE-2-PyP treatment, but mitochondrial membrane potential was unaffected. In addition, MnTE-2-PyP significantly increased $N A D(P)+/ N A D(P) H$ ratios in $P C 3$ and LNCaP cells in a dose-dependent manner, which was mainly due to a reduction of cellular $\mathrm{NAD}(\mathrm{P}) \mathrm{H}$ pool. Correspondingly, we observed a significant decrease of activity in glucose6-phosphate dehydrogenase (G6PD) and 6-phosphogluconate dehydrogenase (6PGD), which are major cellular NADPH producing enzymes in pentose phosphate pathway. A decrease of GSH/GSSG ratios were confirmed in MnTE-2-PyP-treated prostate cancer cells, which may result from the decreased glutathione reductase (GR) activity due to NADPH depletion. We also identified the oxidation of Ser/Thr protein phosphatase 1 beta catalytic subunit (PP1CB), and a decrease of PP1CB activity in MnTE-2-PyP-treated prostate cancer cells. One key protein, pRB, regulates cell cycle progression that is downstream target of PP1CB was hypophosphorylated in MnTE-2-PyP-treated prostate cancer cells. Significant increase of Ki67-negative populations were observed in both PC3 and LNCaP cells but overall cell cycle progression was not altered, which indicates 
interfering cell cycle progression is not the major mechanism of MnTE-2-PyP-induced cell growth inhibition. Besides protein oxidation, MnTE-2-PyP also caused nuclear abnormalities in prostate cancer cells. High $\mathrm{H}_{2} \mathrm{O}_{2}$ levels by MnTE-2-PyP treatment induced nuclear fragmentation in PC3 cells, which could be synergistically enhanced with radiotherapy both in vitro and in vivo. In LNCaP cells, disturbing $\mathrm{H}_{2} \mathrm{O}_{2}$ balance may contribute to the bi-nucleation phenomenon. The increased $\mathrm{H}_{2} \mathrm{O}_{2}$ levels, protein oxidative modifications, mitotic catastrophe, cellular energy metabolism alterations, and $\mathrm{NAD}(\mathrm{P}) \mathrm{H}$ depletion caused by MnTE-2-PyP are all likely factors contributing to prostate cancer cell growth inhibition. 


\section{LIST OF ABBREVIATIONS}

$\begin{array}{ll}\text { 2-DG } & \text { 2-deoxy-D-glucose } \\ \text { 2-NBDG } & \text { 2-(N-(7-Nitrobenz-2-oxa-1,3-diazol-4-yl)Amino)-2-Deoxyglucose } \\ \text { 6PGD } & \text { 6-phosphogluconate dehydrogenase } \\ \text { ADT } & \text { androgen-depravation therapy } \\ \text { APCs } & \text { antigen-presenting cells } \\ \text { BCA } & \text { bicinchoninic acid } \\ \text { BIAM } & \text { (N-(biotinoyl)-N'-(iodoacetyl) ethylenediamine) } \\ \text { BuOE } & \text { MnTnBuOE-2-PyP } \\ \text { CAT } & \text { catalase adenovirus } \\ \text { CMH } & \text { 1-hydroxy-3-methoxycarbonyl-2,2,5,5-tetramethyl pyrrolidine } \\ \text { hydrochloride } \\ \text { EPR }\end{array}$




\begin{tabular}{|c|c|}
\hline FBS & fetal bovine serum \\
\hline G6PD & glucose-6-phosphate dehydrogenase \\
\hline GAPDH & glyceraldehyde 3-phosphate dehydrogenase \\
\hline GPx & glutathione peroxidase \\
\hline GR & glutathione reductase \\
\hline GSH & glutathione \\
\hline HPLC & high performance liquid chromatography \\
\hline $\mathrm{HPrF}$ & human primary prostate fibroblast \\
\hline i.p. & intraperitoneal \\
\hline IAM & iodoacetamide \\
\hline $\mathrm{KGDH}$ & a-ketoglutarate dehydrogenase \\
\hline $\mathrm{IDH} 1$ & isocitrate dehydrogenase 1 \\
\hline $\mathrm{IDH} 3$ & isocitrate dehydrogenase 3 \\
\hline IG & \\
\hline $\mathrm{LDH}$ & lactate dehydrogenase \\
\hline $\mathrm{mCRPC}$ & metastatic castration-resistant prostate cancer \\
\hline $\mathrm{MDH} 2$ & malate dehydrogenase 2 \\
\hline ME1 & malic enzyme 1 \\
\hline $\mathrm{MnP}$ & Mn porphyrin \\
\hline MOI & multiplicity of infection \\
\hline NOXs & NADPH oxidases \\
\hline
\end{tabular}




\begin{tabular}{|c|c|}
\hline NNT & NAM nucleotide transhydrogenase \\
\hline NQO1 & $\mathrm{NAD}(\mathrm{P}) \mathrm{H}$ :quinone acceptor oxidoreductases 1 \\
\hline OCR & oxygen consumption rate \\
\hline OXPHOS & oxidative phosphorylation \\
\hline PC3-Luc & luciferase expressing PC3 \\
\hline PEG & polyethylene glycol \\
\hline PEG-CAT & catalase-polyethylene glycol \\
\hline PEP & phosphoenolpyruvate \\
\hline PMF & primary mouse prostate fibroblast \\
\hline PMSF & phenylmethylsulfonyl fluoride \\
\hline P01 & peroxy Orange 1 \\
\hline PP1CB & Ser/Thr protein phosphatase 1 catalytic subunit beta \\
\hline PP2A & Ser/Thr protein phosphatase $2 \mathrm{~A}$ \\
\hline PPP & Ser/Thr phosphoprotein phosphatase \\
\hline $\mathrm{pRB}$ & retinoblastoma protein \\
\hline ROI & regions of interest \\
\hline Prx & peroxiredoxin \\
\hline RT & radiotherapy \\
\hline SIRT & sirtuin \\
\hline SODs & superoxide dismutases \\
\hline $\mathrm{T} 2 \mathrm{E}$ & MnTE-2-PyP \\
\hline
\end{tabular}




$\begin{array}{ll}\text { TCA } & \text { tricarboxylic acid } \\ \text { TCEP } & \text { tris-(2-carboxyethyl)-phosphine } \\ \text { TGF- } \beta & \text { transforming growth factor beta } \\ \text { Th } & \text { helper T cell } \\ \text { Th1 } & \text { type } 1 \text { helper T cell } \\ \text { Th2 } & \text { type } 2 \text { helper T cell } \\ \text { TMRM } & \text { tetramethylrhodamine, methyl ester } \\ \text { TR } & \text { thioredoxin reductase } \\ \text { Trx } & \text { thioredoxin }\end{array}$




\section{LIST OF TABLES}

Table 1. Primary antibodies used for western blot analysis.

Table 2. Protein peptide targets with reversible redox modifications 


\section{LIST OF FIGURES}

Figure 1. Structures and chemistry of MnTE-2-PyP and MnTnBuOE-2-PyP.

Figure 2. Cellular NADP+/NADPH function.

Figure 3: Cell growth in prostate cancer cell lines treated with PBS or MnTE-2-PyP.

Figure 4: PC3 and LNCaP growth with PBS or MnTE-2-PyP with radiation treatment.

Figure 5: HPrF cells growth with PBS or MnTE-2-PyP treatment.

Figure 6: $\mathrm{H}_{2} \mathrm{O}_{2}$ measurements in prostate cancer cells with MnTE-2-PyP with radiation treatment using the ROS-Glo probe.

Figure 7: $\mathrm{H}_{2} \mathrm{O}_{2}$ measurements in prostate cancer cells with MnTE-2-PyP/MnTnBuOE-2PyP and radiation treatment using the PO1 probe.

Figure 8: Basal superoxide measurement in normal prostate cells and prostate cancer cells using the $\mathrm{CMH}$ spin probe.

Figure 9: Superoxide levels in prostate cancer cells measured with MnPs \pm radiation with DHE.

Figure 10: Comparison of catalase activity in normal prostate cells and prostate cancer cells with MnTE-2-PyP treatment.

Figure 11. MnTE-2-PyP suppressed PC3 cells growth through intracellular $\mathrm{H}_{2} \mathrm{O}_{2}$ levels increase.

Figure 12. MnTE-2-PyP suppressed LNCaP cells growth through intracellular $\mathrm{H}_{2} \mathrm{O}_{2}$ levels increase.

Figure 13: Determination of thiol oxidation in MnTE-2-PyP-treated normal prostate cells and prostate cancer cells. 
Figure 14: Identification of thiol oxidation in MnTE-2-PyP-treated PC3 cells.

Figure 15. Identification of MnTE-2-PyP-induced PP1CB oxidation and PPPs activity measurement.

Figure 16. PP1 activity with catalase overexpression and DTT treatment in PC3 cells.

Figure 17. Phosphorylation levels and expression levels of PPP-regulated proteins.

Figure 18. MnTE-2-PyP enhanced Ki67-negative population in PC3 cells but did not affect cell cycle progression.

Figure 19. Effects of MnTE-2-PyP on PC3 cell cycle progression.

Figure 20. MnTE-2-PyP did not affect Ki67-negative population and cell cycle progression in PC3 tumor cells in vivo.

Figure 21. Effects of MnTE-2-PyP on cell cycle progression in combination with radiation in LNCaP cells.

Figure 22. Nuclear abnormalities of prostate cancer cells treated with MnTE-2-PyP and radiation.

Figure 23. Effects of MnTE-2-PyP with radiation in PC3 tumors in vivo.

Figure 24. Effects of MnTE-2-PyP on cell cycle progression in combination with radiation in TrampC cells.

Figure 24. lodoTMT-switch assay in PC3 cells.

Figure 25. PKM activity was not altered by MnTE-2-PyP treatment in PC3 cells.

Figure 26. Identifying oxidized PKM2 isoforms in PC3 cells with $\mathrm{H}_{2} \mathrm{O}_{2}$ treatment.

Figure 27. MnTE-2-PyP treatment reduced complex 2 expression levels in PC3 cells. 
Figure 28. MnTE-2-PyP treatment altered PC3 cells metabolism in combination with radiation.

Figure 29. MnTE-2-PyP treatment altered LNCaP cells metabolism in combination with radiation.

Figure 30. MnTE-2-PyP and radiation affects cellular ATP levels and radiation affects mitochondrial membrane potential in prostate cancer cells.

Figure 31. MnTE-2-PyP and radiation alone or in combination enhanced glucose uptake in prostate cancer cells.

Figure 32. MnTE-2-PyP and radiation enhanced NAD+/NADH ratios in prostate cancer cells.

Figure 33. MnTE-2-PyP and radiation enhanced NADP+ levels and depleted NADPH pool in prostate cancer cells.

Figure 34. MnTE-2-PyP and MnTnBuOE-2-PyP affected NADP+/NADPH levels in a dosedependent manner.

Figure 35. MnTE-2-PyP depleted NADPH pools in PC3 and LNCaP cells as measured by HPLC and mass spectrometry analysis.

Figure 36. MnTE-2-PyP increased succinate levels in LNCaP cells but did not alter FAD/FADH ${ }_{2}$ levels in PC3 and LNCaP cells as measured by HPLC and mass spectrometry analysis.

Figure 37. Catalase transduction did not rescue NADPH ratios in MnTE-2-PyP-treated prostate cancer cells.

Figure 38. NOX enzymes were not involved in MnTE-2-PyP-induced NADPH levels decrease. 
Figure 39. MnTE-2-PyP resulted in G6PD inhibition in prostate cancer cells.

Figure 40. MnTE-2-PyP resulted in 6PGD inhibition in prostate cancer cells.

Figure 41. MnTE-2-PyP and radiation affect mitochondrial $\mathrm{H} 2 \mathrm{O} 2$ and overall ROS levels in prostate cancer cells.

Figure 42. MnTE-2-PyP and radiation affect GSH and GSSG levels in prostate cancer cells as measured by the glutathione assay kit.

Figure 43. MnTE-2-PyP and radiation affect GSH and GSSG levels in prostate cancer cells identified by HPLC and mass spectrometry analysis.

Figure 44. MnTE-2-PyP may decrease GSH/GSSG ratios in prostate cancer cells by reducing cellular NADPH levels.

Figure 45: The mechanisms by which MnTE-2-PyP suppresses prostate cancer growth.

Figure 46: Radiation increases macrophage infiltration in RM1 tumor samples.

Figure 47: MnTnBuOE-2-PyP reduces immune cell population in RM1 tumor after radiotherapy.

Figure 48: MnTnBuOE-2-PyP greatly reduces metastasis in RM1 tumor after radiotherapy.

Figure 49: MnTE-2-PyP increases macrophage infiltration in PC3 tumors. 


\section{TABLE OF CONTENTS}

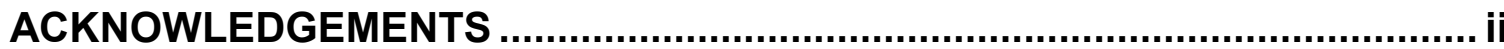

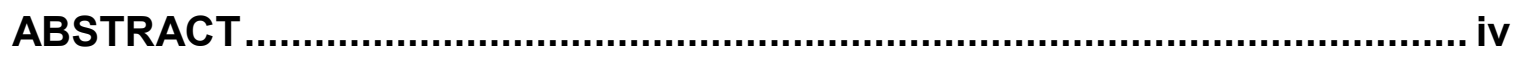

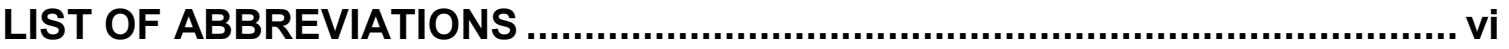

LIST OF TABLES

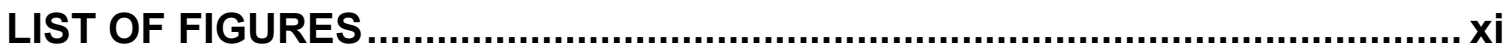

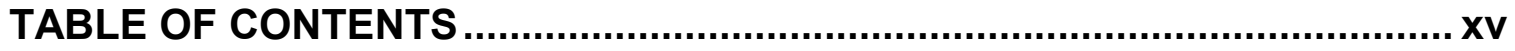

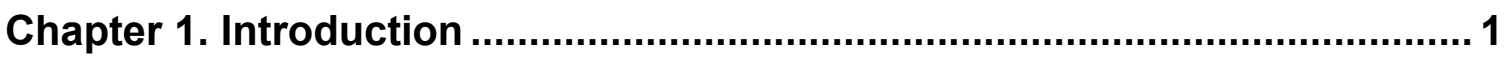

1.1. Prostate cancer and current therapies .................................................... 1

1.2. Oxidative stress and antioxidant defense system in prostate cancer ............. 2

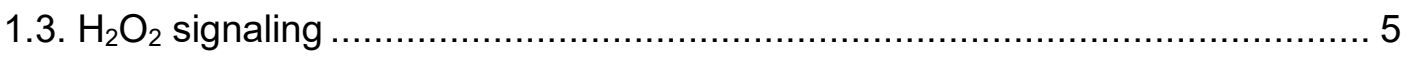

1.4. MnTE-2-PyP and MnTnBuOE-2-PyP in prostate cancer radiotherapy ........... 5

1.5. Normal prostate epithelial cell and prostate cancer cell energy metabolism ..11

1.6. $N A D(H) / N A D P(H)$ metabolism and their relation to prostate cancer .............12

1.7. Rationale, hypothesis and current questions ………............................15

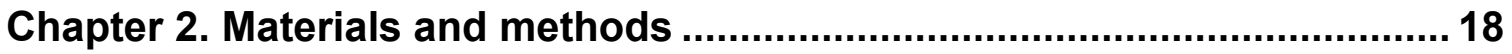

2.1. Cell lines, tissue culture and reagents ................................................18

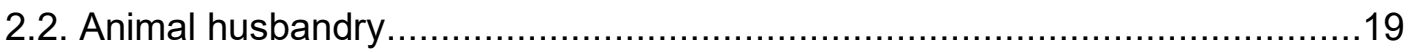

2.3. Orthotopic implantation of PC3 tumor cells .............................................19

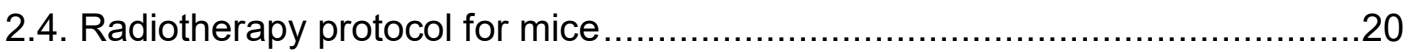


2.5. Tumor harvesting and tumor size measurement.

2.6. Cell proliferation and cell viability measurement.

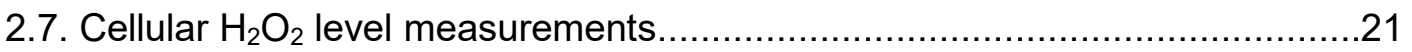

2.8. Cellular superoxide level measurements ................................................22

2.9. Altering intracellular or extracellular catalase activity ...........................23

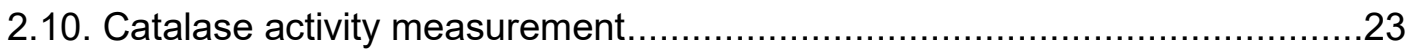

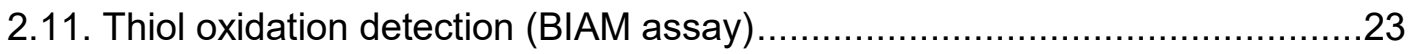

2.12. Reversible thiol modifications detection (lodoTMT-switch assay) ...............24

2.13. Ser/Thr phosphoprotein phosphatase (PPP) activity measurement ............25

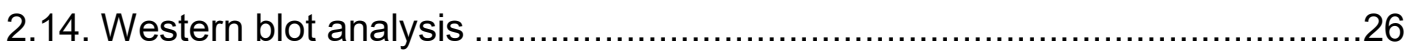

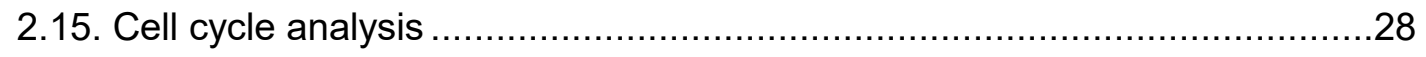

2.16. Nuclear abnormalities and DNA damage imaging ...............................28

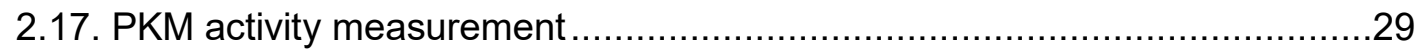

2.18. Oxygen consumption rate (OCR) and extracellular acidification rate (ECAR)

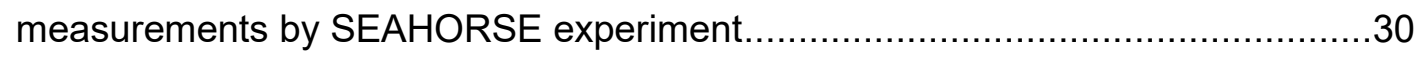

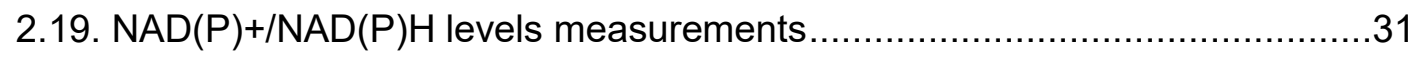

2.20. Mitochondrial overall ROS levels and $\mathrm{H}_{2} \mathrm{O}_{2}$ levels measurements ..............31

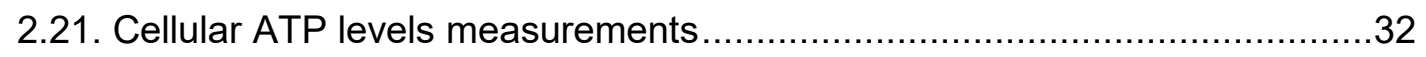

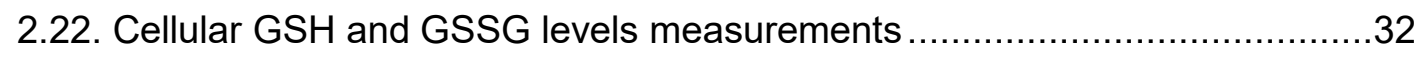

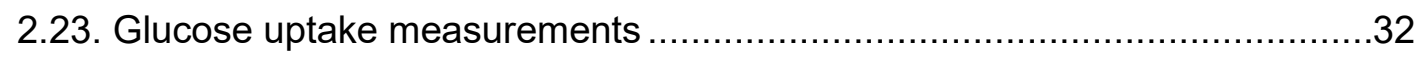

2.24. Mitochondrial membrane potential measurements ................................33 
2.25. Glucose-6-phosphate dehydrogenase (G6PD) activity measurements 33

2.26. 6-phosphogluconate dehydrogenase (6PGD) activity measurements. 34

2.27. Glutathione reductase (GR) activity measurements..... 34

2.28. Cellular metabolites measurements by high performance liquid chromatography (HPLC) and mass spectrometry analysis .35

2.29. Data analysis. 35

Chapter 3. Results 36

3.1. In vitro prostate cancer cell proliferation with MnTE-2-PyP treatment. 36

3.2. In vitro normal prostate cell proliferation with MnTE-2-PyP treatment.

3.3. Intracellular $\mathrm{H}_{2} \mathrm{O}_{2}$ measurements in prostate cancer cells with MnTE-2-PyP treatment.

3.4. Intracellular superoxide levels measurements in normal prostate cells and prostate cancer cells .50

3.5. Catalase activity in normal prostate cells and prostate cancer cells .56

3.6. Intracellular $\mathrm{H}_{2} \mathrm{O}_{2}$ levels alterations affect prostate cancer cell growth .59

3.7. Identifying protein oxidation in normal prostate cells and prostate cancer cells with MnTE-2-PyP treatment (BIAM assay) 66

3.8. MnTE-2-PyP affects PPP-regulated signaling pathways. .78

3.9. MnTE-2-PyP enhances Ki67-negative cells but does not affect overall cell cycle distribution in vivo or in vitro in PC3 cells.

3.10. MnTE-2-PyP enhances Ki67-negative cells and induces cell quiescence or cell death in LNCaP cells 
3.11. MnTE-2-PyP treatment induces nuclear abnormalities in prostate cancer cells in vitro

3.12. MnTE-2-PyP treatment induces nuclear abnormalities in prostate cancer cells in vivo. 101

3.13. MnTE-2-PyP does not induce the Ki67-negative population in TrampC cells .106

3.14. Identifying MnTE-2-PyP-induced reversible thiol modifications in PC3 cells (lodoTMT-switch assay) 109

3.15. MnTE-2-PyP does not affect PKM activity in PC3 cells. .114

3.16. The effects of MnTE-2-PyP on prostate cancer energy metabolism 119

3.17. MnTE-2-PyP and radiation treatment affect prostate cancer cell ATP levels and membrane potential. .133

3.18. The effects of MnTE-2-PyP on prostate cancer cell glucose uptake 137

3.19. The effects of MnTE-2-PyP on NAD(H) and NADP(H) levels in prostate cancer cells

3.20. Investigating potential factors/pathways that result in NADPH depletion....159

3.21. The effects of MnTE-2-PyP on mitochondrial $\mathrm{H}_{2} \mathrm{O}_{2}$ and ROS levels in prostate cancer cells

3.22. The effects of MnTE-2-PyP on GSH and GSSG levels in prostate cancer cells.

3.23. MnTE-2-PyP inhibits GR activity by depleting NADPH pool. 184 
1. The mechanisms by which MnTE-2-PyP suppresses prostate cancer growth 188 2. The combinatorial effects of MnTE-2-PyP and radiation on prostate cancer cells.

3. Future directions ..... 204

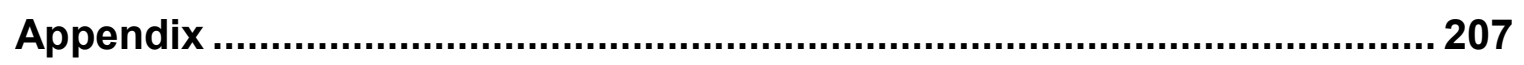

Appendix A: The application of Mn porphyrins in combination with immunotherapy and radiotherapy for treatment of prostate cancer..... 207

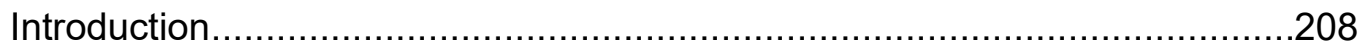

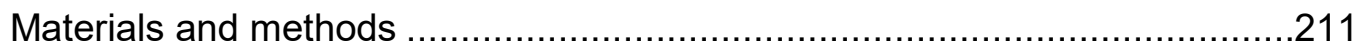

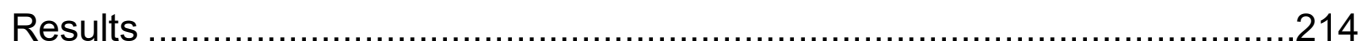

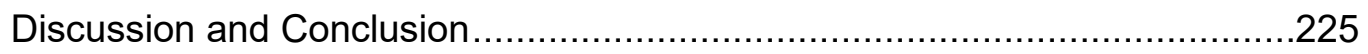

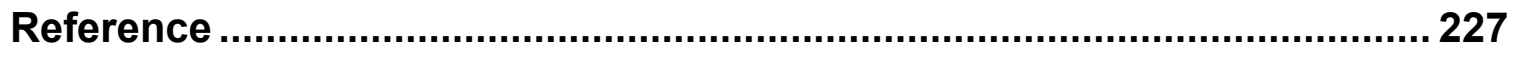




\section{Chapter 1. Introduction}

\subsection{Prostate cancer and current therapies}

Prostate cancer afflicts men worldwide. Currently in the U.S., prostate cancer is the second most common cancer type and third leading cause of cancer-associated death [1, 2]. Known as a disease of aging, cancer is positively linked to patient age. Particularly, for prostate cancer, more than $90 \%$ of the diagnosed patients are 55 years of age or older with the average age of 67 at diagnosis [3]. Though highly related to aging, the natural occurrence of prostate cancer is not well-understood. Large-scale genome-wide association studies have been performed to better understand the genetic origins of prostate cancer [3]. More than 40 genetic aberrations in prostate cancer tumors have been identified, common genes include: AR [4, 5], BRCA [6, 7], ERF [8], RB1 [5, 9], PTEN [10, 11], and TP53 [5, 9]. However, prostate cancer is highly heterogeneous. Besides genetic alterations, multiple foci can be found in one single patients-derived tumor and even within one foci, distinct genomic profiles are identified $[12,13]$. This makes the origin of prostate tumor harder to determine. Other potential prostate carcinogenesis have been investigated, such as race, ethnicity, and geography [1]. It has been shown that as compared to white men, African American men are more likely to develop prostate cancer and the average age at diagnose is younger; however, there is a decreasing trend of prostate cancer cases in Hispanic males during recent years, which showed disparities regarding the origins of country among patients [14-16]. Based on these studies, there is a significant correlation of family prostate cancer history to diagnosed cases, but large variations exist among race, ethnicity, and geography [1, 3].

The majority of diagnosed cases are local or regional prostate cancers, and the

overall 5 year survival rate of prostate cancer is very high (around 95\%) as compared to other cancer types [17]. Currently, there are many available treatment options, including 
surgery, androgen-depravation therapy (ADT), chemotherapy, radiotherapy (RT), and immunotherapy depending on the stage of prostate cancer [17]. During the past decades, ADT has become the most commonly used treatment for prostate cancer; however, even though ADT directly suppresses prostate cancer growth in the beginning, many patients eventually develop castration-resistance and the cancer becomes more aggressive regardless of testosterone levels $[18,19]$.

RT is another primary treatment option for prostate cancer, including internal radiation therapy (brachytherapy) and external beam radiation. It is estimated that over $50 \%$ of the patients will undergo RT with or without other treatments [20]. Ionizing radiation produces free radicals, which results in DNA damage and subsequently halts the fast proliferation of prostate cancer cells. Permanent cell cycle arrest or cell death will occur if DNA repair is not successful $[21,22]$. Though highly efficient, there are several issues with radiotherapy. First of all, RT is able to cure localized prostate cancer, but is not effective against highly advanced or metastatic prostate cancer, even when there is only one single metastatic site [23]. Secondly, RT also damages surrounding normal tissues, which leads to acute and chronic side effects [24]. Finally, some prostate cancer cells are able to survive RT and become radiation-resistant. These cancer cells are not only resistant to further RT, but may also become resistant to other treatments and eventually can result in patient death [25]. Methods have been developed to address the above issues, including using radioprotectors for normal tissue protection [26], use of sophisticated image guidance (IG) techniques in RT to achieve precise targeting [27], and combining radiotherapy with other treatments to achieve curable outcomes [28, 29].

\subsection{Oxidative stress and antioxidant defense system in prostate cancer}

The concept of oxidative stress was coined in 1985 [30], and since then, many studies have been performed to investigate its role in biological systems. Oxidative stress 
is defined as "an imbalance between oxidants and antioxidants in favor of the oxidants, leading to a disruption of redox signaling and control and/or molecular damage" [30, 31]. The most common oxidants are reactive oxygen species (ROS), which includes superoxide radicals $\left(\mathrm{O}_{2}{ }^{--}\right)$, hydrogen peroxide $\left(\mathrm{H}_{2} \mathrm{O}_{2}\right)$, hydroxyl radicals $(\cdot \mathrm{OH})$, peroxynitrate $\left(\mathrm{ONOO}^{-}\right)$and singlet oxygen $\left({ }^{1} \mathrm{O}_{2}\right)$ etc. [32]. Free radicals are an another group of molecules that can overlap with ROS members and induce oxidative stress, e.g. superoxide and $\bullet \mathrm{OH}$ [33]. Although oxidants like $\mathrm{H}_{2} \mathrm{O}_{2}$ and lipid hydroperoxide $(\mathrm{LOOH})$ are not free radicals, they can easily generate free radicals through interactions with other cellular components [34]. Both free radicals and oxidants, if in excess, can cause detrimental effects to cellular macromolecules, such as lipid peroxidation, protein oxidation/modification, and DNA lesions [35-38]. In normal cells, serious oxidative events can lead to cell death and even induce human disease $[39,40]$. Therefore, it is critical to maintain cellular redox balance. In contrast, many studies indicate that prostate cancer has significantly higher oxidative stress markers than normal tissue, with an inconsistent alteration in cellular antioxidant enzymes activities [41].

Antioxidant enzymes play a key role in the antioxidant defense system and many have been around since aerobic respiration began [42]. One of the oldest families is the superoxide dismutases (SODs). SODs are the only proteins that catalyze the dismutation of superoxide into $\mathrm{H}_{2} \mathrm{O}_{2}$ and $\mathrm{O}_{2}$ [43]. Almost all the living organisms have SOD proteins and there are three isoforms of SOD in mammals: Cu/ZnSOD (encoded by the sod1 gene), MnSOD (encoded by the sod2 gene), and extracellular superoxide dismutase (ECSOD, encoded by the sod3 gene) [44]. Each SOD isoform has a distinct cellular distribution and cannot cross membranes: $\mathrm{Cu} / \mathrm{Zn}$ SOD is found primarily in cytosol, while some subcellular compartments including nucleus, lysosome, peroxisome, and the mitochondrial intermembrane space also have Cu/Zn SOD [45]. ECSOD, as its name indicates, is 
secreted into the extracellular space and accounts for majority of plasma SOD [46, 47]. Mn SOD is found exclusively in the mitochondria which is a source of cellular ROS. The unique $\mathrm{Mn}$ metal center distinguishes it from other SODs by being insensitive to cyanide [48]. Since only SODs can catalyze the dismutation of superoxide to $\mathrm{H}_{2} \mathrm{O}_{2}$, these enzymes play a key role in cellular redox signaling pathways and have been implicated in disease progression. Past studies have shown significant alterations of SOD family members in different cancer types including prostate cancer $[41,49,50]$. Dr. Larry Oberley's group identified that increased MnSOD levels are crucial in the suppression of prostate tumor growth, which results in cell cycle interference and induction of senescence or apoptosis $[51,52]$. Although SODs are generally considered as antioxidant enzymes, overexpression of SODs have been shown to suppress prostate tumor growth mainly through increasing cellular $\mathrm{H}_{2} \mathrm{O}_{2}$ levels $[51,53]$.

Catalase, another central antioxidant enzyme that is present in most aerobic organisms, catalyzes the conversion of two $\mathrm{H}_{2} \mathrm{O}_{2}$ molecules into one molecule of $\mathrm{O}_{2}$ and two molecules of $\mathrm{H}_{2} \mathrm{O}$. Thus, catalase detoxifies $\mathrm{H}_{2} \mathrm{O}_{2}$ to produce harmless end products $[54,55]$. Catalase is universally expressed in different human tissues, with very high concentration in liver cells [56]. For subcellular distribution, catalase is primarily expressed in peroxisomes, but also found in the mitochondria and cytosol [57]. Although catalase is not the only enzyme that dismutates $\mathrm{H}_{2} \mathrm{O}_{2}$, the rate of dismutation of $\mathrm{H}_{2} \mathrm{O}_{2}$ by catalase is extremely high and catalase seems to be the major $\mathrm{H}_{2} \mathrm{O}_{2}$-removal enzyme in a situation where $\mathrm{H}_{2} \mathrm{O}_{2}$ levels are overwhelming $[58,59]$. Interestingly, catalase activity have been reported to decline in prostate cancer $[41,60]$.

To protect against oxidative damage, cells and tissues have a network of antioxidant enzymes to remove excess ROS. Besides SODs and catalase, the glutathione (GSH) - glutathione reductase (GR) system and thioredoxin (Trx)-thioredoxin reductase 
(TR) system can also detoxify cellular $\mathrm{H}_{2} \mathrm{O}_{2}$ and handle protein oxidation $[61,62]$. Point mutations or altered activity of these proteins have been reported in prostate cancer cells, but their roles still remain controversial during prostate cancer progression [41, 50, 63-65].

\section{3. $\mathrm{H}_{2} \mathrm{O}_{2}$ signaling}

For a long time, $\mathrm{H}_{2} \mathrm{O}_{2}$ was recognized as a ROS molecule that was responsible for cellular oxidative damage [66]. Recently, $\mathrm{H}_{2} \mathrm{O}_{2}$ 's role in normal cellular signal transduction has been demonstrated [67]. To date, 31 human cellular $\mathrm{H}_{2} \mathrm{O}_{2}$-generating enzymes have been identified [68]. The major cell compartments contributing to $\mathrm{H}_{2} \mathrm{O}_{2}$ production includes the mitochondria, the endoplasmic reticulum, and the peroxisomes [66]. Among the

common ROS, $\mathrm{H}_{2} \mathrm{O}_{2}$ is a relatively stable molecule (about 1 millisecond half-life in a biological system) with decent diffusibility and selective reactivity in the cellular environment, which makes it a perfect ROS signaling molecule $[69,70]$. Currently, the most acknowledged mechanism of $\mathrm{H}_{2} \mathrm{O}_{2}$ acting as a signaling molecule is the direct oxidation of critical thiols on redox-sensitive proteins [71]. $\mathrm{H}_{2} \mathrm{O}_{2}$ oxidizes thiols to the reversible sulfenic acid $(\mathrm{SOH})$ as a mean to regulate cellular signaling. However, in the presence of high concentrations of $\mathrm{H}_{2} \mathrm{O}_{2}$, sulfenic acid can be further oxidized to sulfinic acid $\left(\mathrm{SO}_{2} \mathrm{H}\right)$ or sulfonic acid $\left(\mathrm{SO}_{3} \mathrm{H}\right)$, which are non-reversible, and thus, considered to be oxidative damage. In certain conditions, the oxidation of signaling proteins can occur indirectly by thioredoxin- or peroxiredoxin-based redox relay reactions as well $[72,73]$.

\subsection{MnTE-2-PyP and MnTnBuOE-2-PyP in prostate cancer radiotherapy}

The unique function and important role of SODs in maintaining cellular redox balance have stimulated the design of SOD mimics. Several types of natural or synthetic SOD mimics have therapeutic effects in different diseases [74-77]. Radiation is a classic model of damage caused by oxidative stress. Enhancing SOD activity has been shown to 
protect normal tissues from radiation damage $[78,79]$. One type of the most potent SOD mimics is $\mathrm{Mn}$ porphyrin (MnP), which typically consists of a planar porphyrin ring that contains a Mn center [80]. Currently, two Mn(III) porphyrins, MnTE-2-PyP ${ }^{5+}$ (BMX-010, $\mathrm{Mn}(\mathrm{III})$ meso-tetrakis(N-ethylpyridinium-2-yl)porphyrin]) and MnTnBuOE-2-PyP ${ }^{5+}$ (BMX001, [Mn(III) meso-tetrakis(N-(2'-n-butoxyethyl)pyridinium-2-yl)porphyrin]), have been shown to provide radioprotection (Figure 1A, B) [81]. The dismutation of superoxide is a two-step process as shown in Figure 1C. According to Dr. Batinic-Haberle's group, as long as the reduction potential $\left(E_{1 / 2}\right)$ of the $M n$ center is appropriate for the exchange of electrons with superoxide, the superoxide dismutation can be completed by the Mn porphyrin compounds $[82,83]$. To achieve the optimal efficacy of dismutating superoxide, MnTE-2-PyP and MnTnBuOE-2-PyP are designed to be in the +3 oxidation state of the Mn center, which allows them to keep both redox-active ability and biological stability [84].

As compared to SOD enzymes, MnTE-2-PyP and MnTnBuOE-2-PyP have lower $\mathrm{k}_{\text {cat }}\left(\mathrm{O}_{2}{ }^{--}\right)$values $\left(7.76 \times 10^{9} \mathrm{M}^{-1} \mathrm{~S}^{-1}\right.$ and $7.73 \times 10^{9} \mathrm{M}^{-1} \mathrm{~S}^{-1}$, respectively) than native SOD enzymes $\left(8.84 \sim 9.30 \times 10^{9} \mathrm{M}^{-1} \mathrm{~S}^{-1}\right)$, but the $E_{1 / 2}$ is quite close, all ranging from $+220 \sim 300 \mathrm{mV}$ [81, 85]. In addition, MnTE-2-PyP and MnTnBuOE-2-PyP not only scavenge superoxide, but also scavenge peroxynitrite (ONOO-) and carbonate radical $\left(\mathrm{CO}_{3}{ }^{--}\right)$, which grants them more biological functions [86]. As described above, SODs have distinct cellular distribution, and Mn SOD exclusively localizes in the mitochondria. To mimic the function of MnSOD, MnTE-2-PyP and MnTnBuOE-2-PyP have the ability to accumulate in mitochondria. Studies on mouse heart tissue show that a single intraperitoneal injection of MnTE-2-PyP (10 mg/Kg) can result in $2.95 \pm 1.24 \mathrm{ng} / \mathrm{mg}$ protein accumulation of drug in mitochondria after 4 hours treatment [87]. Based on in vitro studies using S. cerevisiae and E. coli, MnTE-2-PyP also shows a higher accumulation in the mitochondria than in the cytosol, which is directly linked to lipophilicity [85, 88]. Interestingly, several studies 
based upon different MnPs show that MnPs accumulate more in tumor than in normal tissue, which is similar to what our lab has observed (data not shown) [89-91].

Based on previous studies, both MnTE-2-PyP and MnTnBuOE-2-PyP can mimic all three kinds of SODs due to their distributions in different cellular locations including mitochondria, nucleus, and cytosol etc. The brain, salivary glands, bone marrow, and liver are protected in different animal models with MnTE-2-PyP or MnTnBuOE-2-PyP treatment [92-94]. Our lab's recent work shows that MnTE-2-PyP and MnTnBuOE-2-PyP protect mouse normal tissue during radiotherapy [95-97]. We found that several proteins involved in the anti-oxidant defense system, e.g. NQO1, MnSOD, and NRF2 had higher expression in MnTE-2-PyP-treated normal prostate fibroblasts cells as compared to PBS treatment alone or with radiation. The NRF2 signaling pathway plays a critical role in MnTE-2-PyPmediated radioprotection. In addition, not only the MnSOD levels were enhanced in P3158 cells (human prostate fibroblasts) under MnTE-2-PyP treatment, MnSOD activity was also increased most likely due to sirtuin-mediated deacetylation [97]. Similar in vitro results were also obtained with MnTnBuOE-2-PyP treatment. We identified that MnTE-2-PyP inhibited the transforming growth factor beta (TGF- $\beta$ ) mediated fibroblast activation pathway by suppressing TGF- $\beta$ receptor 2 expression, which led to profibrotic markers down-regulation and inhibited senescence [96]. Other than NRF2 and TGF- $\beta$ pathways, Dr. Batinic-Haberle suggests that NF-KB inhibition is essential to the radioprotective effects of Mn porphyrins [81].

Early studies have focused on the radioprotective effects of MnPs on normal tissues, but in recent years, tumor suppressing effects of MnPs are being explored. Dr. Tome's group found that MnTE-2-PyP or MnTnBuOE-2-PyP increased dexamethasoneinduced mitochondrial ROS and caused oxidation of the mitochondrial glutathione pool, which induced apoptosis in WEHI7.2 murine thymic lymphoma cells, but not in normal 
lymphocytes [98]. Similar results were also obtained in B cell lymphoma with MnTE-2-PyP treatment [81]. In a breast tumor mouse model, a relatively low dose of MnTE-2-PyP (2 mg/kg/day) did not trigger tumor suppressive effects; however, a much higher dose of MnTE-2-PyP (15 mg/kg/day) led to significant tumor growth suppression [89]. The tumor suppression effects were further improved with radiation and/or ascorbate treatment. For prostate cancer, our lab demonstrated that MnTE-2-PyP and MnTnBuOE-2-PyP can suppress prostate cancer growth both in vitro and in vivo with or without radiation. In these cancer-related studies, MnP's tumor suppressive effects are highly associated with ROS, especially enhanced $\mathrm{H}_{2} \mathrm{O}_{2}$ levels $[90,98]$. In addition, MnTE-2-PyP and MnTnBuOE-2PyP, or other MnPs, selectively suppress tumor growth and enhance radiation effects while protecting normal cells from radiation damage $[99,100]$. We found that both MnTE2-PyP and MnTnBuOE-2-PyP enhanced lipid peroxidation in prostate cancer cells under radiation treatment but protected normal tissues from radiation-induced lipid peroxidation [95]. Although several pathways have been postulated to address the differences observed between normal and cancer cells, the mechanisms remain unknown [81], which will be the major focus of this thesis. 
Figure 1. Structures and chemistry of MnTE-2-PyP and MnTnBuOE-2-PyP. (A) Structure of MnTE-2-PyP or BMX-010 (CASRN 219818-60-7, Molecular Weight 965.13). (B) Structure of MnTnBuOE-2-PyP or BMX-001 (CASRN 1379783-91-1, Molecular Weight 1253.55). (C) The dismutation of superoxide by MnPs in a two-step process. 
A

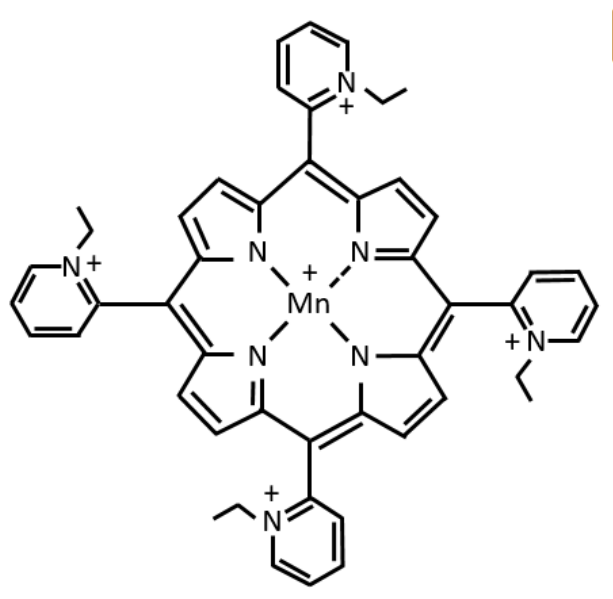

MnTE-2-PyP
B

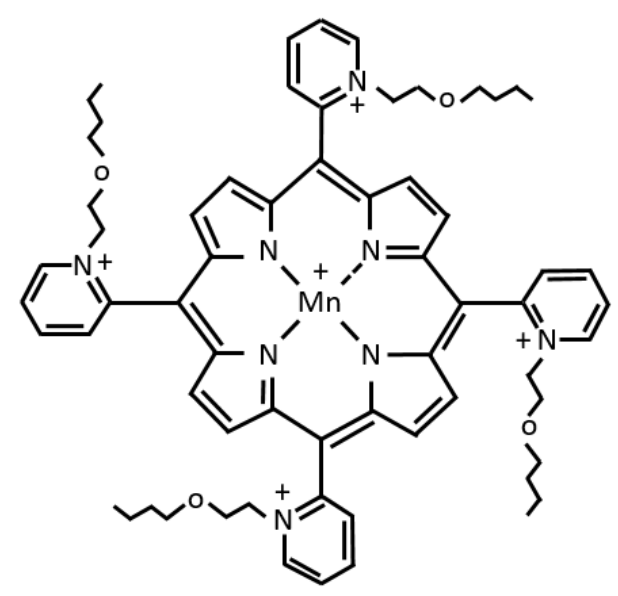

MnTnBuOE-2-PyP

C

$$
\begin{aligned}
& \mathrm{Mn}^{\mathrm{III}} \mathrm{P}^{5+}+\mathrm{O}_{2}{ }^{--} \rightleftharpoons \mathrm{Mn}^{\mathrm{II}} \mathrm{P}^{4+}+\mathrm{O}_{2} \\
& \mathrm{Mn}^{11} \mathrm{P}^{4+}+\mathrm{O}_{2}{ }^{--}+2 \mathrm{H}^{+} \rightleftharpoons \mathrm{Mn}^{\text {IIIP5+ }}+\mathrm{H}_{2} \mathrm{O}_{2}
\end{aligned}
$$




\subsection{Normal prostate epithelial cell and prostate cancer cell energy metabolism}

The prostate cell has a unique energy metabolism pattern. Normal prostate epithelial cells accumulate and secrete extremely high levels of zinc and citrate, which is about 5 1000 fold higher than cells of other tissue types [101, 102]. The extremely high intracellular zinc level inhibits the mitochondrial aconitase (m-aconitase) activity and prevents the conversion of citrate to isocitrate in tricarboxylic acid (TCA) cycle [103]. Therefore, in normal prostate epithelial cells, both TCA cycle and oxidative phosphorylation (OXPHOS) are inhibited. This process is very costly because $\sim 65 \%$ of the energy production will be lost if citrate does not enter the TCA cycle as compared to the complete oxidation of glucose. As a consequence, normal prostate epithelial cells can retain high citrate production at the cost of being in an energy-inefficient state by adapting to a unique metabolism style.

Alteration of energy metabolism is a universal feature of most cancer cell types, and normally, the Warburg effect is observed [104, 105]. Unlike other cancer cell types, prostate cancer cells undergo a complete metabolic switch from energy-inefficient glycolysis to energy efficient TCA cycle and OXPHOS $[101,102,106,107]$. The drastic decreased zinc levels in prostate cancer cells re-activate the m-aconitase and enable the proper function of the TCA cycle $[103,107]$, which provides the energy required for malignant transformation. Interestingly, there are also reports indicating that early stage

prostate tumors use a lipid-based energy source, while metastasized prostate cancer can rely on the Warburg effect, but these phenomenon are poorly elucidated [108, 109]. 


\section{6. $N A D(H) / N A D P(H)$ metabolism and their relation to prostate cancer}

$\mathrm{NAD+/NADH}$ and NADP+/NADPH are crucial redox couples in regulating cellular redox balance, energy metabolism, macromolecular metabolism, and signaling pathways. NADP phosphatase and NADH kinase are responsible for the conversion between these two couples and deficiency or imbalance of either of the two redox couples are associated with pathological disorders [110].

NAD exists in two forms: NAD+, acting as electron acceptor, and $\mathrm{NADH}$, acting as electron donor. The interconversion between NAD+ and its reduced forms NADH can occur in cellular energy metabolic pathways such as glycolysis, pentose phosphate pathway, TCA cycle, and OXPHOS [111]. Due to the central role of NAD+/NADH in energy metabolism, its distribution is tightly regulated in the mitochondria. The outer mitochondrial membrane is very porous, enabling NADH to diffuse freely into the intermembrane space; however, the inner mitochondrial membrane is impermeable to $\mathrm{NADH}$. This results in a significant difference of NAD+/NADH ratio between cytosol and mitochondria matrix. The cytosolic NAD+/NADH ratios range between 60 and 700 in most cell types, and mitochondrial $\mathrm{NAD}+\mathrm{NADH}$ ratios are strictly regulated from 7 to 10 [112]. This huge difference of NAD+/NADH ratio is mainly based upon different NADH concentrations in cytosol and mitochondrial matrix [113-116].

The major source of mitochondrial NADH is the TCA cycle. Three enzymes in the TCA cycle that can directly reduce NAD+ to NADH are: isocitrate dehydrogenase 3 (IDH3), a-ketoglutarate dehydrogenase $(\mathrm{KGDH})$, and malate dehydrogenase (MDH2). On the other hand, cytosolic NADH is mainly produced during glycolysis by glyceraldehyde 3phosphate dehydrogenase (GAPDH). In certain conditions, cytosolic NADH can also be produced by lactate dehydrogenase (LDH), which is a reversible process from lactate to pyruvate $[111,112,117]$. 
$\mathrm{NAD}(\mathrm{H})$ plays an important role in prostate cancer progression due to its metabolism pattern alteration. Reducing the NAD+ pool by overexpressing the NADase, CD38, can directly suppress prostate cancer proliferation [118]. The NAD-dependent sirtuin (SIRT) family of proteins are essential in NAD+ recycling and oxidative stress handling. One group of researchers has shown that increasing SIRT3, the mitochondrialocated NAD-dependent deacetylase, can inhibit prostate tumor progression by attenuating the Wnt/ $\beta$-catenin pathway [119]. However, cytosol-located sirtuin protein, SIRT2, has been reported to be down-regulated in castrate-resistant prostate cancer cells [120]. In addition, SIRT1 is reported to be upregulated in prostate cancer cells and contributes to oxidative stress response and DNA damage repair [121]. Another NAD+ regenerating protein, NAMPT, is upregulated in early prostate cancer progression, and knockdown of the NAMPT sensitizes prostate cancer cells to oxidative stress [122, 123]. Depleting the NAD+ pool by the NAMPT specific inhibitor, APO866, not only reduced prostate cancer cells growth, but also sensitized them to radiotherapy [124].

The NADP+/NADPH redox couple plays an important role in cellular antioxidant defense [125]. As an essential cofactor of GR and TR, NADPH re-activates both enzymes and facilitates the $\mathrm{H}_{2} \mathrm{O}_{2}$ removal through $\mathrm{GSH}$-dependent glutathione peroxidase (GPx) and Trx-dependent peroxiredoxin (Prx) (Figure 2A). Unlike the cellular NAD+/NADH ratio, the cellular NADP+/NADPH ratio is much lower, normally below 1 , and can be as low as 0.001 in certain organs, like the liver $[111,116,125-127]$. In addition, the mitochondrial NADP+/NADPH is normally more than $95 \%$ reduced under physiological conditions [128].

The major source of cellular NADPH is the pentose phosphate pathway, which is produced by glucose-6-phosphate dehydrogenase (G6PD) and 6-phosphogluconate dehydrogenase (6PGD), while the only source and determinant factor for cellular NADP+ de novo synthesis is NAD kinase (NADK) [111, 129]. For cytosolic NADPH, cytosolic 
isocitrate dehydrogenase 1 (IDH1) catalyzes a similar reaction as mitochondrial IDH3 (use NAD+ as substrate) to generate NADPH. In HEK293T cells, it was found that cytosolic malic enzyme 1 (ME1) is the major contributor to NADPH production [130]. On the other hand, mitochondrial NADPH can be produced by mitochondrial IDH (IDH2) and ME (ME3). Another significant contributor to mitochondrial NADPH is NAM nucleotide transhydrogenase (NNT), which directly converts NADH to NADPH [111]. As was observed for $\mathrm{NAD+} / \mathrm{NADH}$, the mitochondrial membrane is impermeable to $\mathrm{NADP}+/ \mathrm{NADPH}$, and the isocitrate- $\alpha-K G$ shuttle is able to exchange cytosolic/mitochondrial NADPH by transforming isocitrate to $\alpha-K G$ and NADP+ to NADPH, which is an important source of cytosolic NADPH.

Although NADPH is an important molecule to cellular antioxidant defense, excessive NADPH can also induce oxidative stress via NADPH oxidases (NOXs, Figure 2B). Studies show that the aggressiveness and metastatic ability of prostate cancer cells are associated with intracellular ROS levels, which partially results from NOXs [131, 132]. In addition, overexpression of Nox1 in prostate cancer cells enhanced cell growth and angiogenicity [133], but down-regulation of Nox5 led to growth arrest and apoptosis [132].

Besides regulating redox balance, NADP+/NADPH is also critical to cellular reductive synthesis [134]. Anabolic reactions, e.g. fatty acid synthesis, cholesterol synthesis, and steroidogenesis, all require NADPH in several key steps [134-136]. Prostate cancer cells possess distinct alterations in acyl-chain composition between benign and malignant tissue [137]. Another study also reported that aggressive cancer cells, including prostate cancer cells, robustly incorporate exogenous fatty acids for the synthesis of signaling lipids [138]. This indicates that fatty acid synthesis may play an important role in cancer cell adaption to the aggressiveness transition. In addition, NADPH also plays an indispensable role in de novo synthesis of building blocks for 
macromolecular synthesis, e.g. amino acid synthesis and one carbon metabolism [139141]. As the major cellular reducing power carrier, NADPH is extremely important in cell metabolism and maintaining the NADP+/NADPH ratio and is, therefore, necessary for cell survival and proliferation.

\subsection{Rationale, hypothesis and current questions}

How Mn porphyrins have opposite effects on tumor cells as compared to normal tissues during radiotherapy remains an unanswered question. Based on the $\mathrm{Mn}$ porphyrins superoxide dismutation reaction, we hypothesize that MnTE-2-PyP inhibits prostate cancer growth by increasing cellular $\mathrm{H}_{2} \mathrm{O}_{2}$ levels, which results in oxidative damage to cellular macromolecules including proteins, DNA, and lipids. The oxidation of key proteins that are involved in cell proliferation lead to alterations of multiple signaling pathways and contribute to the prostate cancer growth inhibition. In addition, the $\mathrm{H}_{2} \mathrm{O}_{2}$ increase directly affects mitochondria function where the superoxide dismutation reaction occurs, and this causes energy metabolism decrease, which also leads to prostate cancer growth inhibition.

To test the hypothesis, there are several key questions to answer: 1) whether MnTE-2-PyP alone is able to significantly increase $\mathrm{H}_{2} \mathrm{O}_{2}$ in different prostate cancer cell lines and induce oxidative damage; 2 ) if protein oxidation occurs, what are the central pathways contributing to the cell growth inhibition; 3) does MnTE-2-PyP or Mn porphyrins suppress prostate cancer cell proliferation without affecting normal cells; 4) does MnTE2-PyP alters energy metabolism in prostate cancer; 5) does MnTE-2-PyP inhibit prostate cancer growth in vivo by the same mechanism as in vitro? 
Figure 2. Cellular NADP+/NADPH function. (A) NADP+/NADPH function as cofactors in antioxidant defense systems. (B) NADP+/NADPH function as cofactors in prooxidant NOX enzymes. 
A

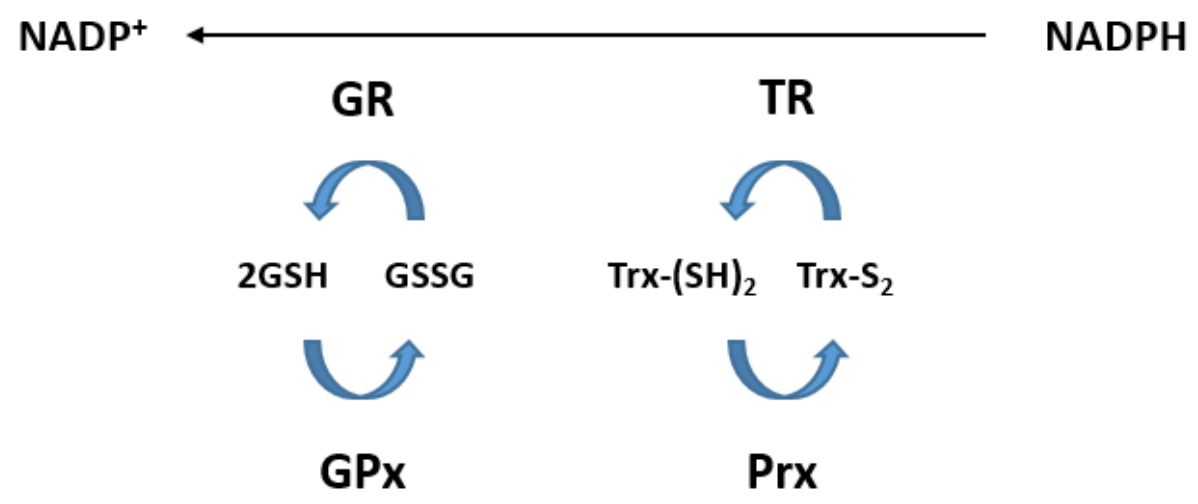

$\mathrm{H}_{2} \mathrm{O}_{2} \longrightarrow \mathrm{H}_{2} \mathrm{O}$

B

$\mathrm{NADPH}+2 \mathrm{O}_{2} \stackrel{\text { NOXs }}{\longrightarrow} \mathrm{NADP}^{+}+2 \mathrm{O}_{2} \cdot$ 


\section{Chapter 2. Materials and methods}

\subsection{Cell lines, tissue culture and reagents}

PC3 (human prostate cancer cell line), LNCaP (human prostate cancer cell line), and TrampC (mouse prostate cancer cell line) cells were purchased from American Type Culture Collection ${ }^{\circledR}$ (Manassas, VA, USA). C42B and C81 cells (human prostate cancer cell line) were gifts from Dr. Ming-Fong Lin at the University of Nebraska Medical Center. P3158 (Immortalized human prostate fibroblast cells) were obtained from Dr. Tyson McDonald at Hampton University. The RM1 (mouse prostate cancer cell line) cells were a gift from Dr. Cook Leah at the University of Nebraska Medical Center. For PC3, LNCaP, RM1, C42B, C81, and P3158 cell lines, cells were cultured in RPMI-1640 medium (Hyclone, Logan, UT, USA) with the addition of $10 \%$ fetal bovine serum (FBS) and $1 \%$ penicillin/streptomycin. For TrampC cells, cells were cultured in DMEM medium with the addition of $(+)$-dehydroisoandrosterone $(10 \mathrm{nM}), 10 \%$ insulin, 10\% fetal bovine serum and $1 \%$ penicillin/streptomycin. Normal human primary prostate fibroblast $(\mathrm{HPrF})$ cells were purchased from ScienCell Research Laboratories. HPrF cells were cultured in Fibroblast Medium (ScienCell Research Laboratories, Carlsbad, CA, USA) with the addition of $10 \%$ FBS, $1 \%$ penicillin/streptomycin, and fibroblast growth factors on poly-l-lysine coated flasks $\left(2 \mu \mathrm{g} / \mathrm{cm}^{2}\right)$. Primary mouse prostate fibroblast (PMF) cells were isolated from the prostate tissue of 6 8-week-old C57BL/6J mice. In brief, prostatic tissues were minced and digested with collagenase, then washed with $\mathrm{HBSS} \mathrm{w} / \mathrm{Ca}^{2+} \& \mathrm{Mg}^{2+}$. Digestion were terminated with $\mathrm{HBSS}$ w/o $\mathrm{Ca}^{2+} \& \mathrm{Mg}^{2+}$. PMF cells were cultured in DMEM (Hyclone, Logan, UT, USA) media with the addition of $10 \%$ fetal bovine serum, $1 \%$ penicillin/streptomycin and $1 \%$ nonessential amino acids. All cells were cultured in a 37 ${ }^{\circ} \mathrm{C}$ incubator containing $95 \%$ air and $5 \% \mathrm{CO}_{2}$. 
MnTE-2-PyP (T2E) and MnTnBuOE-2-PyP (BuOE) were gifts from Dr. James Crapo at National Jewish Health, Denver, CO, USA. PBS was used to dissolve MnTE-2PyP and MnTnBuOE-2-PyP; therefore, the same volume of PBS was added to the growth media as a control whenever MnTE-2-PyP or MnTnBuOE-2-PyP was used.

\subsection{Animal husbandry}

Male C57BI/6 mice (Charles River Laboratories, Wilmington, MA, USA and Taconic, Rensselaer, NY, USA) and athymic nude mice (Charles River Laboratories) were used for experiments. All mice were exposed to a 12 hours light/12 hours dark cycle and fed and watered ad libitum at the University of Nebraska Medical Center (UNMC, Omaha, NE, USA). All experimental protocols were reviewed and approved by the UNMC Institutional Animal Care and Use Committee (20-019-03FC).

\subsection{Orthotopic implantation of PC3 tumor cells}

The constitutive luciferase expressing PC3 cells (PC3-Luc) were purchased from Applied Biological Materials Inc. (Richmond, BC, Canada). For PC3-Luc tumor implantation, athymic nude mice were used. Mice were anesthetized by continuous flow of $2.5 \%$ isoflurane with oxygen using a mouse anesthesia machine. A 1:1 mixture of PC3Luc cells and Matrigel (Corning, Tewksbury, MA, USA) was injected into the dorsal prostatic lobe (50 $\mu \mathrm{L}$ mixture containing 2 million PC3-Luc cells). The peritonium was closed with absorbable catgut sutures (Surgical Specialties, Tijuana, Mexico) and the skin was closed with wound clips (Thomas Scientific, Swedesboro, NJ, USA). Then, 0, 6, 24, and 48 hours after surgery, buprenorphine $(0.1 \mathrm{mg} / \mathrm{kg}$, Reckitt Benckiser Healthcare (UK) Ltd., Hull, UK) was administrated intraperitoneally. The health condition of all mice was monitored daily, and 10 days after surgery, the wound clips were removed. 


\subsection{Radiotherapy protocol for mice}

The tumor size was measured every week after the 5th week of orthotopic implantation by intraperitoneal D-Luciferin injection (100 mg/kg, PerkinElmer, Waltham, MA, USA) using Xenogen IVIS Spectrum bioluminescence imaging system (PerkinElmer, MA, USA). Five weeks post-surgery, mice were divided into 2 groups: PBS or MnTE-2PyP with radiation (2 Gy per day, for five sequential days). PBS or MnTE-2-PyP ( $5 \mathrm{mg} / \mathrm{kg}$ ) was administrated intraperitoneally 24 hours before radiation and three times every week until mice were sacrificed. Tumor sizes of each mouse were compared by calculating the regions of interest $(\mathrm{ROI})$ based on luminescence intensity. Tumors were CT imaged to verify size and location, then irradiated with image-guided X-rays using the Small Animal Radiation Research Platform (Xstrahl, Suwanee, GA, USA).

\subsection{Tumor harvesting and tumor size measurement}

Animals were sacrificed 2 weeks post-radiation treatment. The width and length of the excised tumor were measured with calipers and the volume was estimated according to the formula: [(width $)^{2} \times$ length/2]. The tumor was divided into 3 parts: one part was flash frozen and stored at $-80{ }^{\circ} \mathrm{C}$ for western blot analyses; one part was fixed in $4 \%$ formalin followed by $70 \%$ ethanol, and these tissues were paraffin embedded (Tissue Science Facility, UNMC). Sections were cut and placed on slides for immunostaining. The third part of the tumor was minced in ice-cold PBS into pieces ranging from 1 to $3 \mathrm{~mm}^{3}$. The tumor pieces with PBS were centrifuged at $100 \mathrm{~g}$ for $5 \mathrm{~min}$ at room temperature. The supernatants were discarded, and the pelleted tumor pieces were incubated with Collagenase I (1mg/mL, Life Technologies, Eugene, OR, USA) and DNAse (100 Kunitz, Worthington, $\mathrm{OH}, \mathrm{USA}$ ) for 1 hour at $37^{\circ} \mathrm{C}$. The digested tumor pieces were triturated about 20 times with different sizes of plastic serologic pipets, and then tumor pieces were strained through a $70 \mu \mathrm{m}$ strainer followed by $35 \mu \mathrm{m}$ strainer (Thermo Fisher Scientific, 
Rochester, NY, USA). Single cells were washed with PBS and cell numbers were determined using a Coulter counter (Beckman Coulter, Indianapolis, IN, USA). The cell viability was determined by Trypan Blue assay. Tumor cells collected from the third part were used for Ki67 staining.

\subsection{Cell proliferation and cell viability measurement}

Cell growth was measured by daily cell counting for all tested conditions. Cell viability was assessed by Trypan Blue staining using a hemocytometer under bright field microscope.

For live/dead cell imaging, cells were treated with calcein-acetoxymethyl (calceinAM, 50 pM, Invitrogen, Carlsbad, CA, USA) and Hoechst ( $1 \mu \mathrm{g} / \mathrm{mL}$, AnaSpec, Fremont, CA, USA) for $20 \mathrm{~min}$ at $37^{\circ} \mathrm{C}$ in the dark. Cells were trypsinized and centrifuged at $500 \mathrm{~g}$ for $3 \mathrm{~min}$, and then resuspended in RPMI-1640 medium. Cell viability and nuclear morphology were observed using a LEICA DM4000 B LED fluorescent microscope (Leica, Plymouth, MN, USA). For image quantification, the average calcein-AM signal per cell was calculated.

\subsection{Cellular $\mathrm{H}_{2} \mathrm{O}_{2}$ level measurements}

To measure overall $\mathrm{H}_{2} \mathrm{O}_{2}$ levels, a ROS-Glo kit (Promega, Madison, WI, USA) was used as described previously [142]. Cells with different conditions were seeded on Nunclon $^{\mathrm{TM}} 96$ Flat White Plates (Thermo Fisher Scientific, Rochester, NY, USA). $\mathrm{H}_{2} \mathrm{O}_{2}$ levels were measured according to the manufacturer's instructions. The luminescence signal of $\mathrm{H}_{2} \mathrm{O}_{2}$ was recorded by an Infinite M200 Pro Plate Reader (Tecan, Männedorf, Switzerland).

To measure intracellular $\mathrm{H}_{2} \mathrm{O}_{2}$ levels, a Peroxy Orange 1 (PO1, Thermo Fisher Scientific, Rochester, NY, USA) probe was used as described previously [95]. Cells were 
treated with PO1 $(15 \mu \mathrm{M})$ for 1 hour in the dark at $37^{\circ} \mathrm{C}$. The fluorescence was detected by a Leica DM4000B B LED fluorescent microscope with the Ex/Em at $555 \mathrm{~nm} / 565 \mathrm{~nm}$. An average of five images were taken for each condition. The average intensity per cell was calculated based on a minimum number of 100 cells for each condition and the analysis was performed using ImageJ (v1.50i).

\subsection{Cellular superoxide level measurements}

For basal cellular superoxide level measurement, the 1-hydroxy-3methoxycarbonyl-2,2,5,5-tetramethyl pyrrolidine hydrochloride $(\mathrm{CMH})$ probe was used. Cells were seeded in $60 \mathrm{~mm}$ culture dish for different conditions. On the day of the experiment, culture media was removed and the $\mathrm{CMH}$ probe $(200 \mu \mathrm{M}$ in PBS) was added to cells for $30 \mathrm{~min}$ at $37^{\circ} \mathrm{C}$. After incubation, the majority of the $\mathrm{CMH}$ solution was removed with around $100 \mu \mathrm{l}$ solution remaining. Cells were gently scraped and a $50 \mu \mathrm{l}$ cell suspension was subjected into EPR glass capillary tube and then placed into the EPR spectrometer. The peak and trough signals were used to calculate superoxide levels and were normalized to cell number or protein concentration.

For superoxide levels measurement with different conditions, the dihydroethidium (DHE) probes were used. Cells were seeded in flasks and on the day of the experiment, cells were trypsinized and enumerated. Cells were stained with DHE $(5 \mu \mathrm{M})$ for $20 \mathrm{~min}$ at $37^{\circ} \mathrm{C}$ in the dark and then subjected to flow cytometric analysis using a LSRII Green 532 Flow Cytometer (BD Biosciences, San Jose, CA, USA). In order to measure superoxide specifically, 405/570 nm excitation/emission was used. Data was analyzed using FACSDiVa analysis software (v8.0.2, BD Biosciences, San Jose, CA, USA). 


\subsection{Altering intracellular or extracellular catalase activity}

The catalase adenovirus transduction was performed as described previously [142]; cells were serum-starved in RPMI- 1640 media supplemented with $2 \%$ fetal bovine serum for 6 hours with corresponding multiplicity of infection (MOI) of Empty vector (ViraQuest Inc., North Liberty, IA, USA) or Ad5CMVCAT catalase adenovirus (produced by the University of lowa Viral Vector Core, lowa city, IA, USA). Cells were then placed in $10 \%$ fetal bovine serum media for an additional 48 hours.

\subsection{Catalase activity measurement}

Catalase-polyethylene glycol (PEG-CAT, Sigma-Aldrich, Darmstadt, Germany) was used to scavenge extracellular $\mathrm{H}_{2} \mathrm{O}_{2}$. For controls, the calculated weight of polyethylene glycol (PEG, Sigma-Aldrich, Darmstadt, Germany) that corresponds to PEGCAT was used. Different doses from 1 to $1000 \mathrm{U} / \mathrm{mL}$ were tested in cell culture with 1 or 24 hours pre-treatment.

For catalase activity gels, cells were lysed and centrifuged at $4{ }^{\circ} \mathrm{C}$ for $7 \mathrm{~min}$ at $12,000 \mathrm{~g}$. Supernatants were collected and loaded onto 10\% Mini-PROTEAN TGX precast gels (Bio-Rad, Hercules, CA, USA). The gel was run at $100 \mathrm{~V}$ for 2 hours on ice and then rinsed with distilled $\mathrm{H}_{2} \mathrm{O}$ three times for 10 min each. The gel was incubated in $0.003 \%$ $\mathrm{H}_{2} \mathrm{O}_{2}$ for 10 min and rinsed twice with distilled $\mathrm{H}_{2} \mathrm{O}$ quickly. The staining solution (2\% ferric chloride and $2 \%$ potassium ferricyanide in distilled water) was poured onto the gel immediately after rinsing, and achromatic bands were indicative of catalase activity. Gel images were inverted, and densitometry of the bands were performed using ImageJ.

\subsection{Thiol oxidation detection (BIAM assay)}

The BIAM (N-(biotinoyl)-N'-(iodoacetyl) ethylenediamine) thiol oxidation detection assay was performed as described previously [142]. In brief, cell lysates were incubated 
with a reduced thiol-specific probe, BIAM (Life Technologies, Eugene, OR, USA) for 30 min in the dark at room temperature. Then, Pierce ${ }^{\text {TM }}$ Streptavidin Agarose Resin (Thermo Fisher Scientific, Rockford, IL, USA) was added to each sample for 1 hour at room temperature. The protein-resin complexes were washed 4 times with binding buffer (PBS $+0.1 \%$ SDS) and then heated at $75{ }^{\circ} \mathrm{C}$ for $10 \mathrm{~min}$. The supernatant was loaded in a Bolt ${ }^{\mathrm{TM}} 4 \%-12 \%$ Bis-Tris Plus gel (Invitrogen, Hercules, CA, USA) and stained by GelCode ${ }^{\circledR}$ Blue Stain Reagent (Thermo Scientific, Rockford, IL, USA).

To perform mass spectrometry analysis, protein-rich regions were cut from the gels of PBS or MnTE-2-PyP $(30 \mu \mathrm{M})$ treatment at the same molecular weight region. An overnight in-gel trypsin digestion was performed followed by peptide clean-up using $\mu \mathrm{C} 18$ ZipTip (Millipore, Burlington, MA, USA). Each sample was resuspended in $0.1 \%$ formic acid and injected through Eksigent cHiPLC column $(75 \mu \mathrm{m} \times 15 \mathrm{~cm}$ ChromXP C18-CL 3 um 120 Å, Eksigent Technologies, Dublin, CA, USA) onto 6600 TripleTOF (AB Sciex, Framingham, MA, USA) by typical gradient $2 \% \sim 60 \%$ acetonitrile in $60 \mathrm{~min}$. The database search parameters were the following: database, uniprotswissprot; taxonomy, human; search method, thorough. The search results were filtered by comparing proteins identity of bands at same molecular weight in both conditions.

\subsection{Reversible thiol modifications detection (lodoTMT-switch assay)}

For reversible thiol modification detection, the lodo-TMT switch assay was performed [143]. In brief, cells were pre-treated with $1 \mathrm{mM}$ Tris-(2-carboxyethyl)phosphine (TCEP, 1mM, Thermo Fisher Scientific, Rockford, IL) or iodoacetamide (IAM, $50 \mathrm{mM}$, Thermo Fisher Scientific, Rochester, NY). Cell lysates were desalted by Zeba spin desalting columns (Pierce, Rockford, IL) and incubated with $1 \mathrm{mM} \mathrm{TCEP}$ at $55^{\circ} \mathrm{C}$ for 30 minutes in the dark. Cell lysates were cleaned again by Zeba columns. The liquid portion was collected and incubated with lodoTMT at $37^{\circ} \mathrm{C}$ in the dark for 1 hour. Proteins were 
precipitated by a solution containing $500 \mu \mathrm{l}$ methanol, $100 \mu \mathrm{l}$ chloroform, and $300 \mu \mathrm{l} \mathrm{H}_{2} \mathrm{O}$ centrifuged at $13,500 \mathrm{~g}$ for 10 minutes. Proteins were digested in trypsin activated by ammonium bicarbonate overnight. The next day, digested proteins were dried by the vacuum concentrator and purified by a Pierce C18 spin column (Pierce, Rockford, IL). To enrich target proteins, proteins were incubate with anti-TMT resin (Thermo Fisher Scientific, Rockford, IL) overnight at $4{ }^{\circ} \mathrm{C}$. Protein peptides were eluted by TMT elution buffer (Thermo Fisher Scientific, Rockford, IL), and lyophilized by the vacuum concentrator. Samples were re-suspended in $25 \mu \mathrm{l}$ of $5 \%$ acetonitrile/ $0.1 \%$ formic acid. For mass spectrometry analysis, $1 \sim 5 \mu \mathrm{l}$ solution was used each time.

The percentage of protein thiol modification was calculated based on the abundance of lodoTMT-labeled peptides and total peptides. Two types of labeling were performed for two independent experiments as described above: 126 131 lodoTMT labeling and 126 lodoTMT/Non-labeled TMT labeling. The 126 131 lodoTMT labeling provided accurate results of the relative percentage of reversible thiol modifications among PBS and MnTE-2-PyP-treated samples; the 126 lodoTMT/Non-labeled TMT labeling provided less accurate but a larger target pool. Peptides that contained the same type of modifications from both experiments were identified based on the criteria of $>75 \%$ consistency, and then statistical significance of these modifications were calculated by Perseus using single-sample T-test (v1.6.5.0, Max Planck Institute for Biochemistry, Planegg, Germany).

\subsection{Ser/Thr phosphoprotein phosphatase (PPP) activity measurement}

Cells were treated with PBS or MnTE-2-PyP $(30 \mu \mathrm{M})$ for 48 hours. Cells were scraped in $0.5 \mathrm{~mL}$ protein phosphatase lysis buffer $(20 \mathrm{mM}$ imidazole- $\mathrm{HCl}, 2 \mathrm{mM}$ EDTA, $2 \mathrm{mM}$ EGTA, $1 \mathrm{mM}$ benzamidine, $1 \mathrm{mM}$ PMSF, and protease inhibitor, $\mathrm{pH}=7.4$ ). Cells were then sonicated for 3 cycles ( 5 seconds on/cycle) at $40 \%$ amplitude by the Model 120 
Sonic Dismembrator (Thermo Fisher Scientific, Rockford, IL, USA). Cell lysates were spun at $12,000 \mathrm{~g}$ for $7 \mathrm{~min}$ and the supernatant was isolated. The protein concentration was normalized to $1 \mathrm{mg} / \mathrm{mL}$. For PP1 activity measurement, the Ser/Thr protein phosphatase 2A (PP2A) activity was inhibited by $2 \mathrm{nM}$ okadaic acid (Abcam, Cambridge, MA, USA) for 30 min. This concentration efficiently inhibits PP2A activity but not PP1 [144]. The PP1 and total PPP activity was quantified by Ser/Thr protein phosphatase Assay Kit 1 (Millipore Sigma, Billerica, MA, USA). The hydrolysis of phospho-Thr peptide was detected by Malachite green solution and measured by the Infinite M200 Pro Plate Reader at $620 \mathrm{~nm}$.

\subsection{Western blot analysis}

Cells were homogenized and protein concentrations were measured by the Bradford method. Lysed proteins of each sample were separated by electrophoresis on a Bolt ${ }^{\mathrm{TM}} 4 \%-12 \%$ Bis-Tris Plus gel and transferred onto nitrocellulose membranes using an iBlot Transfer Stack (Invitrogen, Carlsbad, CA, USA). After blocking with 5\% non-reduced fat milk or $5 \%$ BSA in TBST for 1 hour, the membranes were incubated overnight at $4{ }^{\circ} \mathrm{C}$ with the following primary antibody (for all western blot analysis, more details are included in Table 1). On the next day, the membrane was washed three times with TBST for 10 min each time, and then the membrane was used incubated with goat anti-rabbit $\lg G(H+L)$ cross-adsorbed secondary antibody, $\operatorname{HRP}(1: 10,000)$ or goat anti-mouse lgG $(\mathrm{H}+\mathrm{L})$ crossadsorbed secondary antibody, $\operatorname{HRP}(1: 10,000)$ (Invitrogen, Carlsbad, CA, USA) at room temperature for 1 hour. The membrane was washed three times with TBST for 10 min each time and visualized by using Pierce ${ }^{\mathrm{TM}}$ ECL Western Blotting Substrate (Thermo Fisher Scientific, Rockford, IL, USA). Each band was quantified via ImageJ software, and the value was normalized to total protein as a loading control by Ponceau (Sigma-Aldrich, Darmstadt, Germany). 
Table 1. Primary antibodies used for western blot analysis

\begin{tabular}{c|c}
\hline Primary antibody(dilution,host) & Primary antibody manufacture \\
\hline PP1CB (1:500, rabbit) & Cell Signaling Technology, Danvers, MA, USA \\
\hline cyclin D1 (1:10,000, rabbit) & Abcam, Cambridge, MA, USA \\
\hline phospho-cyclin D1 Thr 286 (1:1000, rabbit) & Cell Signaling Technology, Danvers, MA, USA \\
\hline pRB ( $1: 1000$, rabbit) & Cell Signaling Technology, Danvers, MA, USA \\
\hline phospho-pRB Ser780 (1:1000, rabbit) & Cell Signaling Technology, Danvers, MA, USA \\
\hline p16 (1:5000, rabbit) & Abcam, Cambridge, MA, USA \\
\hline p21 (1:5000, rabbit) & Abcam, Cambridge, MA, USA \\
\hline PKM2 (1:1000, rabbit) & Abcam, Cambridge, MA, USA \\
\hline Total OXPHOS Rodent WB Antibody Cocktail & Cell Signaling Technology, Danvers, MA, USA \\
\hline
\end{tabular}




\subsection{Cell cycle analysis}

On the day of analysis, cells were pelleted by centrifuging at $500 \mathrm{~g}$ at $4{ }^{\circ} \mathrm{C}$ and then washed twice with PBS. For 4',6-diamidino-2-phenylindole (DAPI)/Ki67 staining, cells were resuspended in $100 \mu \mathrm{l}$ PBS, and $10 \mu \mathrm{l} \mathrm{Ki67-FITC} \mathrm{(Abcam,} \mathrm{Cambridge,} \mathrm{MA,} \mathrm{USA)}$ antibody was added for every 1 million cells. After 30 min incubation at room temperature in the dark, cells were washed with PBS then DAPI $(1 \mu \mathrm{g} / \mathrm{mL}$, Sigma-Aldrich, Darmstadt, Germany) was added. Cells were then incubated at room temperature for $15 \mathrm{~min}$. In order to quantify the staining, $355 / 450 \mathrm{~nm}$ excitation/emission was used for DAPI, $488 / 530 \mathrm{~nm}$ excitation/emission was used for Ki67-FITC. The flow cytometry analysis was performed on a BD LSRII Flow Cytometer. The Ki67-negative population threshold was determined based on a DAPI-only staining control. Data were analyzed using FACSDiVa analysis software.

Similarly, the RNA levels were determined using pyronin $(4 \mu \mathrm{g} / \mathrm{mL}$, Acros Organics, Geel, Belgium) and Hoechst (10 $\mu \mathrm{g} / \mathrm{mL}$, BD Biosciences, San Jose, CA, USA) staining. Cells were treated with a mixture of both stains for $30 \mathrm{~min}$ in the dark at room temperature, and then underwent flow cytometry analysis. To quantify staining, the 355/450 nm excitation/emission was used for Hoechest, while 488/582 nm excitation/emission was used for pyronin.

\subsection{Nuclear abnormalities and DNA damage imaging}

Cells were seeded in media containing PBS or MnTE-2-PyP $(30 \mu \mathrm{M})$ for 24 hours and then irradiated with 2 or 10 Gy of radiation. In some conditions, catalase transduction was applied alone or in combination with other treatments. After 72 hours, cells were trypsinized and resuspended in $200 \mu \mathrm{FBS}$. Cells were spun onto slides at $800 \mathrm{~g}$ for $3 \mathrm{~min}$ using a SHANDON Cytospin 3 Cytocentrifuge (Shandon, Woburn, MA, USA). Slides with 
cells were dried for $15 \mathrm{~min}$ at room temperature and then fixed in $4 \%$ paraformaldehyde for another $15 \mathrm{~min}$. Slides were stored in a preservative $(0.02 \%$ NaAzide in PBS $)$ at $4{ }^{\circ} \mathrm{C}$. PC3 tumor cells isolated from mice were processed similarly after cytospin.

To visualize nuclear abnormalities, slides were washed twice with PBS, 10 min each. Fixed cells were mounted in $25 \mu \mathrm{L}$ ProLong Gold antifade reagent with DAPI (Life Technologies, Eugene, OR, USA) for $30 \mathrm{~min}$ and then imaged using the LEICA DM4000 B LED fluorescent microscope.

The $\mathrm{yH} 2 \mathrm{AX}$ antibody was used as a marker for DNA double strand breaks in PC3 tumor sections. Formalin-fixed paraffin-embedded tissue samples were deparaffinized and heat-induced antigen retrieval was performed. Then tumor samples were permeabilized $(0.1 \%$ Triton $X-100$ in $0.1 \%$ sodium citrate) and blocked with $2 \%$ BSA. Samples were incubated with the anti-gamma H2A.X (phospho S139) antibody (1:2000, Abcam, Cambridge, MA, USA) overnight at $4{ }^{\circ} \mathrm{C}$ and then with goat anti-rabbit $\lg \mathrm{G}(\mathrm{H}+\mathrm{L})$ Superclonal $^{\mathrm{TM}}$ Secondary Antibody, Alexa Fluor 488 for 1 hour at room temperature (1:500, Thermo Fisher Scientific, Rochester, NY, USA).

\subsection{PKM activity measurement}

To measure overall total pyruvate kinase activity levels, the Pyruvate Kinase Activity Assay kit (Sigma-Aldrich, Darmstadt, Germany) was used. On the day of the experiment, cells trypsinized and normalized to same cell numbers. For each condition, 100,000 cells were collected and centrifuged under $15,000 \mathrm{~g}$ for $10 \mathrm{~min}$. To prepare the sample solution, 5,000 cells were used for each condition and $45 \mu$ Pyruvate Kinase Assay Buffer. The $50 \mu \mathrm{l}$ reaction mixture was made according to the company protocol and added to each condition. The $50 \mu \mathrm{l}$ sample solution and $50 \mu \mathrm{l}$ reaction mixture was mixed and the initial absorbance was recorded 2 3 minutes after mixing at $570 \mathrm{~nm}$, and successive readings were recorded every $5 \mathrm{~min}$. Measurements were continuously taken 
until the value of the most active samples are greater than the value of the highest standard. Then the average pyruvate kinase activity was calculated based on the standard curve.

\subsection{Oxygen consumption rate (OCR) and extracellular acidification rate (ECAR) measurements by SEAHORSE experiment}

Mitochondrial OCR was measured using a XF96 Analyzer (Seahorse Biosciences, Santa Clara, CA, USA). Cells were seeded in media containing PBS or $30 \mu \mathrm{M}$ MnTE-2PyP. After 24 hours, some groups of cells were exposed to 2 Gy radiation. After another 48 hours, one day before experiment, cells were re-seeded at a density of 25,000 cells/ well in a 96-well Seahorse plate and incubated at $37^{\circ} \mathrm{C}$ with $5 \% \mathrm{CO}_{2}$ overnight. On the day of experiment, the cells were washed with Mitostress assay medium (free of serum and sodium bicarbonate), and the medium was replaced with $180 \mu \mathrm{l}$ of assay medium. Plates were incubated in a non- $\mathrm{CO}_{2}$ incubator for 1 hour prior to analysis, Oligomycin, FCCP, and Rotenone/Antimycin were injected through the ports A, B, and C, respectively, and mitochondrial OCR was measured. The data were normalized to total cell protein content as measured by Bradford assay.

For ECAR, the same XF96 Analyzer was used. Cells were seeded in media containing PBS or 30 MM MnTE-2-PyP. After 24 hours, some groups of cells were exposed to $2 \mathrm{~Gy}$ radiation. After another 48 hours, one day before experiment, cells were re-seeded at a density of 25,000 cells/ well in a 96 -well Seahorse plate and incubated at $37^{\circ} \mathrm{C}$ with $5 \% \mathrm{CO}_{2}$ overnight. On the day of experiment, the cells were washed with Glycostress assay medium (mediafree of glucose and pyruvate; supplemented with $2 \mathrm{mM}$ GlutaMAX), and the medium was replaced with $180 \mu \mathrm{l}$ of assay medium. Plates were incubated in a non- $\mathrm{CO}_{2}$ incubator for 1 hour prior to analysis. Glucose, Oligomycin, and 2-deoxy-Dglucose (2-DG) were injected through the ports $A, B$, and C, respectively, and ECAR was 
measured. The data were normalized to total cell protein content as measured by Bradford assay.

\subsection{9. $N A D(P)+/ N A D(P) H$ levels measurements}

On the day of experiment, cells were trypsinized and 100,000 cells were used for the assay. Cells were lysed by $100 \mu$ lysis buffer $(50 \mu$ l of PBS $+50 \mu$ l of $0.2 \mathrm{~N} \mathrm{NaOH}$ solution with $1 \%$ dodecyltrimethylammonium bromide). Then each sample was divided equally into two $50 \mu$ parts. For the $N A D(P)+$ group, $25 \mu$ of $0.4 \mathrm{~N} \mathrm{HCl}$ was added in each condition and samples were heated at at $60{ }^{\circ} \mathrm{C}$ for $15 \mathrm{~min}$; for $\mathrm{NAD}(\mathrm{P}) \mathrm{H}$ group, samples were only heated at at $60^{\circ} \mathrm{C}$ for $15 \mathrm{~min}$. All samples were then cooled at room temperature for $10 \mathrm{~min}$. Then, $25 \mu$ of $0.5 \mathrm{M}$ Trizma base was added to $\mathrm{NAD}(\mathrm{P})+$ group and $50 \mu \mathrm{l}$ of $\mathrm{HCl} /$ Trizma solution was added to $\mathrm{NAD}(\mathrm{P}) \mathrm{H}$ group. The $\mathrm{NAD}(\mathrm{P})+/ \mathrm{NAD}(\mathrm{P}) \mathrm{H}-\mathrm{Glo}^{\mathrm{TM}}$ Detection Reagent (Promega, Madison, WI, USA) was prepared according to the company protocol. Each sample was then mixed with equal volume of detection reagent $(100 \mu \mathrm{l})$ and the luminescence was recorded using a Infinite M200 Pro Plate Reader.

\subsection{Mitochondrial overall ROS levels and $\mathrm{H}_{2} \mathrm{O}_{2}$ levels measurements}

On the day of experiment, cells were trypsinized and $1 \times 10^{6}$ cells were used for each condition. For mitochondrial ROS detection, the MitoSOX probe (Thermo Fisher Scientific, Rochester, NY, USA) was used. Cells were resuspended in $\mathrm{HBSS}\left(\mathrm{w} / \mathrm{Ca}^{2+}\right.$ and $\left.\mathrm{Mg}^{2+}\right)+5 \mu \mathrm{M}$ Mitosox and incubated at $37^{\circ} \mathrm{C}$ for $10 \mathrm{~min}$ in the dark. Cells were then washed three times and prepared for FACS. The Ex/Em was 510/580 nm. The LSRII Green 532 Flow Cytometer was used to measure fluorescent intensity and data was analyzed using FACSDiVa analysis software.

For mitochondrial $\mathrm{H}_{2} \mathrm{O}_{2}$ detection, the MitoPY-1 probe was used (Tocris, Minneapolis, MN, USA). Cells were resuspended in HBSS $\left(\mathrm{w} / \mathrm{Ca}^{2+}\right.$ and $\left.\mathrm{Mg}^{2+}\right)+5 \mu \mathrm{M}$ 
MitoPY-1 and incubated at $37^{\circ} \mathrm{C}$ incubator for $40 \mathrm{~min}$ in the dark. Cells were then washed twice and prepared for FACS. The Ex/Em was 488/530 nm. The LSRII Green 532 Flow Cytometer was used to measure fluorescent intensity and data was analyzed using FACSDiVa analysis software.

\subsection{Cellular ATP levels measurements}

Cells were treated with different conditions. On the day of experiment, cells were trypsinized and 200,000 cells were used for each condition. The method used for extracting cellular ATP has previously been described by Yang et al.[145]. In brief, $1 \mathrm{ml}$ of boiling water was added to each sample and samples were heated in the boiling water for $3 \mathrm{~min}$. The cell suspension was then centrifuged at $12,000 \mathrm{~g}$ for $7 \mathrm{~min}$ at $4^{\circ} \mathrm{C}$, and $20 \mu \mathrm{l}$ of the supernatant was mixed with $180 \mu$ detection solution provided by the ATP Determination Kit (Molecular Probes, Eugene, OR, USA). The luminescence that indicates cellular ATP from each sample was recorded by an Infinite M200 Pro Plate Reader.

\subsection{Cellular GSH and GSSG levels measurements}

To measure overall GSH and GSSG levels, the GSH/GSSG-Glo kit (Promega, Madison, WI, USA) was used. Cells with different conditions were seeded in Nunclon ${ }^{\mathrm{TM}}$ 96 Flat White Plates overnight. The next day GSH or GSSG levels were measured according to the manufacturer's instructions. The luminescence signal of GSH or GSSG was recorded by an Infinite M200 Pro Plate Reader as described above.

\subsection{Glucose uptake measurements}

On the day of experiment, cells were trypsinized and 600,000 cells were used for each condition. For glucose uptake measurement, the 2-( $\mathrm{N}-(7-\mathrm{Nitrobenz-2-oxa-1,3-diazol-}$ 4-yl)Amino)-2-Deoxyglucose (2-NBDG, Thermo Fisher Scientific, Rochester, NY, USA) probe was used. Cells were resuspended in RPMI1640 complete media + 2 $\mu$ M 2-NBDG 
and incubated at $37{ }^{\circ} \mathrm{C}$ for $10 \mathrm{~min}$ in the dark. Cells were then washed once with media and prepared for FACS using the Ex/Em of 467/542 nm. The LSRII Green 532 Flow Cytometer was used to measure fluorescent intensity and data was analyzed using FACSDiVa analysis software v8.0.2 as described above.

\subsection{Mitochondrial membrane potential measurements}

On the day of experiment, cells were trypsinized and 600,000 cells were used for

each condition. For mitochondrial membrane potential measurement, the tetramethylrhodamine, methyl ester (TMRM, Thermo Fisher Scientific, Rochester, NY, USA) probe was used. Cells were resuspended in RPMI-1640 complete media $+20 \mathrm{nM}$ TMRM and incubated at $37^{\circ} \mathrm{C}$ for $10 \mathrm{~min}$ in the dark. Cells were then washed once with media and prepared for FACS. The Ex/Em was 560/580 nm. The LSRII Green 532 Flow Cytometer was used to measure fluorescent intensity and data was analyzed using FACSDiVa analysis software v8.0.2 as described above.

\subsection{Glucose-6-phosphate dehydrogenase (G6PD) activity measurements}

The G6PD Activity Assay (Cell Signaling Technology, Danvers, MA, USA) was performed according to the company protocol. In brief, on the day of experiment, cells were washed with ice cold PBS and scraped with $500 \mu \mathrm{l} 1 \mathrm{xCell}$ lysis buffer with $1 \mathrm{mM}$ phenylmethylsulfonyl fluoride (PMSF). Cells were then sonicated for 5 cycles ( 5 seconds on/cycle) at $40 \%$ amplitude by the Model 120 Sonic Dismembrator and spun at $14,000 \mathrm{~g}$ for $10 \mathrm{~min}$ at $4{ }^{\circ} \mathrm{C}$. The protein concentration was determined by bicinchoninic acid (BCA) reagent (Thermo Fisher Scientific, Rochester, NY, USA) and normalized to $0.5 \mathrm{mg} / \mathrm{ml}$ each sample. For each condition, $15 \mu$ g proteins (30 $\mu$ lysates) were mixed with $70 \mu \mathrm{l}$ detection buffer. The reaction mixture was incubated in $37{ }^{\circ} \mathrm{C}$ for $10 \mathrm{~min}$ and the 
fluorescence was determined the Infinite M200 Pro Plate Reader with Ex/Em at $540 \mathrm{~nm} / 590 \mathrm{~nm}$.

\subsection{6-phosphogluconate dehydrogenase (6PGD) activity measurements}

The 6PGD Activity Assay (Abcam, Cambridge, MA, USA) were performed according to the company protocol. In brief, on the day of the experiment, $1 \times 10^{6}$ cells were collected in $100 \mu \mathrm{l}$ ice-cold 6PGD Assay Buffer. Cells were incubated on ice for $10 \mathrm{~min}$ and spun at $10,000 \mathrm{~g}$ for $20 \mathrm{~min}$ at $4^{\circ} \mathrm{C}$. The supernatant was transferred to a new tube. For each condition, $20 \mu \mathrm{l}(200,000$ cells $)$ was used and mixed with $30 \mu \mathrm{l}$ 6PGD Assay Buffer and $50 \mu$ l Detection Solution provided by the assay kit. The reaction mixture was incubated in $37^{\circ} \mathrm{C}$ for $45 \mathrm{~min}$ and the absorbance was recorded every 2 min by the Infinite M200 Pro Plate Reader at 460nm.

\subsection{Glutathione reductase (GR) activity measurements}

The Glutathione Reductase Activity Assay (Abcam, Cambridge, MA, USA) were performed according to the company protocol. In brief, on the day of the experiment, $1 \times 10^{6}$ cells were collected in $100 \mu \mathrm{l}$ ice-cold GR Assay Buffer. Cells were spun at $15,000 \mathrm{~g}$ for 10 $\min$ at $4{ }^{\circ} \mathrm{C}$. The supernatant was transferred to a new tube. To completely remove original $\mathrm{GSH}$, cells were treated with $10 \mu \mathrm{l}$ of $3 \% \mathrm{H}_{2} \mathrm{O}_{2}$, and incubated at room temperature for 5 min. To remove excess $\mathrm{H}_{2} \mathrm{O}_{2}$, cells were treated with $10 \mu \mathrm{l}$ catalase, and incubated at room temperature for $5 \mathrm{~min}$. For each condition, $50 \mu \mathrm{l}(500,000$ cells $)$ was used and mixed with $50 \mu \mathrm{l}$ of Detection Solution provided by the assay kit. The reaction mixture was incubated in room temperature for $20 \mathrm{~min}$ and the absorbance was recorded every 2 min by the Infinite M200 Pro Plate Reader at 405nm. 


\subsection{Cellular metabolites measurements by high performance liquid chromatography (HPLC) and mass spectrometry analysis}

Cellular metabolomics analyses have been described before [146]. In brief, cells were seeded on $75 \mathrm{~mm}^{2}$ dishes and treated in accordance with the treatment groups. Two hours before the collection of metabolites, the culture medium was replaced with fresh medium. Polar metabolites were extracted using $80 \%$ methanol and then analyzed with LC-MS/MS using the selected reaction monitoring method with positive/negative ion polarity switching on a Xevo TQ-S mass spectrometer (Waters, Milford, MA, USA). Peak areas were integrated using Skyline open-source software and were normalized to the respective DNA concentrations. In this thesis, the NAD+, NADH, NADP+, NADPH, FAD, $\mathrm{FADH}_{2}, \mathrm{GSH}, \mathrm{GSSH}$, and succinate levels were measured.

\subsection{Data analysis}

GraphPad Prism 8.3.1 was used for all the statistical analyses. Mean and standard deviation values from three independent experiments were used for statistical analysis for all the experiments performed. Unless otherwise described, significant differences between the groups were determined by a one-way ANOVA test followed by a post hoc Tukey's test for multiple comparisons or a student's t-test. 


\section{Chapter 3. Results}

\subsection{In vitro prostate cancer cell proliferation with MnTE-2-PyP treatment}

Our lab has previously shown that MnTE-2-PyP treatment alone can enhance PC3 tumor-implanted mice survival rate and further increase radiotherapy efficacy [95]. To determine whether MnTE-2-PyP itself has universal anti-tumor effects in different prostate cancer cell lines, we treated PC3 (an aggressive androgen-independent human prostate cancer cell line), LNCaP (an androgen-dependent human prostate cancer cell line), TrampC cells (an aggressive mouse prostate cancer cell line) with MnTE-2-PyP \pm radiation (Figure 3). We found that MnTE-2-PyP alone significantly decreased prostate cancer cell growth by $20 \sim 40 \%$ in all prostate cancer cell lines after 96 hours of treatment. Consistent with previous in vivo data, radiation with MnTE-2-PyP further reduced cell growth as compared to single treatment at the 96 hour time point (Figure 4). 
Figure 3: Cell growth in prostate cancer cell lines treated with PBS or MnTE-2-PyP. Human and mouse prostate cancer cells were treated with PBS or $30 \mu \mathrm{M}$ MnTE-2-PyP and cell numbers were enumerated every day for 7 consecutive days. Data represent mean \pm SD from at least three independent experiments. ${ }^{*} p<0.05$ compared to PBS treatment. 

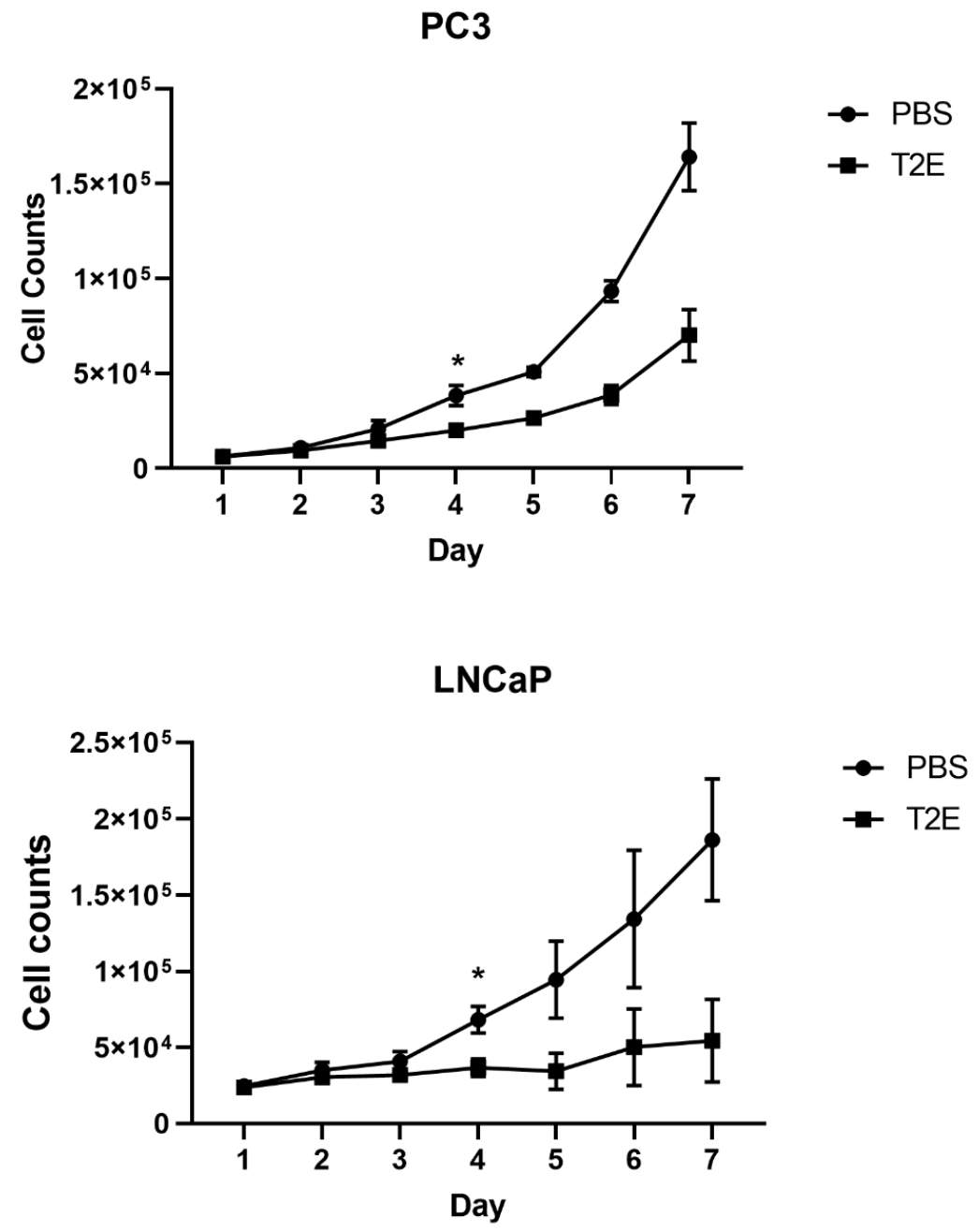

TrampC

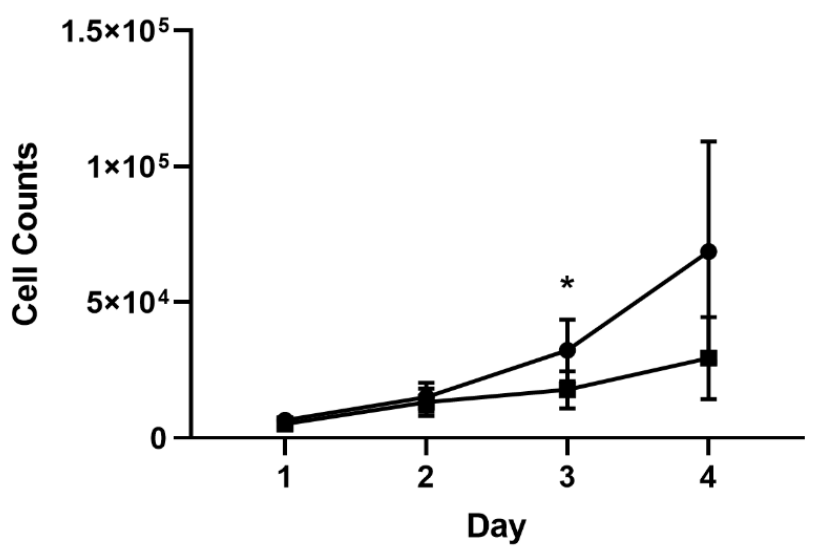

$\rightarrow$ PBS

$\rightarrow \mathrm{T} 2 \mathrm{E}$ 
Figure 4: PC3 and LNCaP growth with PBS or MnTE-2-PyP with radiation treatment. Both PC3 and LNCaP cells were treated with PBS or $30 \mu \mathrm{M}$ MnTE-2-PyP \pm 2 Gy radiation, cell numbers were counted after 96 hours treatment. Data represent mean \pm SD from at least three independent experiments. ${ }^{*} p<0.05$ compared to PBS treatment. \#p $<0.05$ compared to MnTE-2-PyP treatment. 

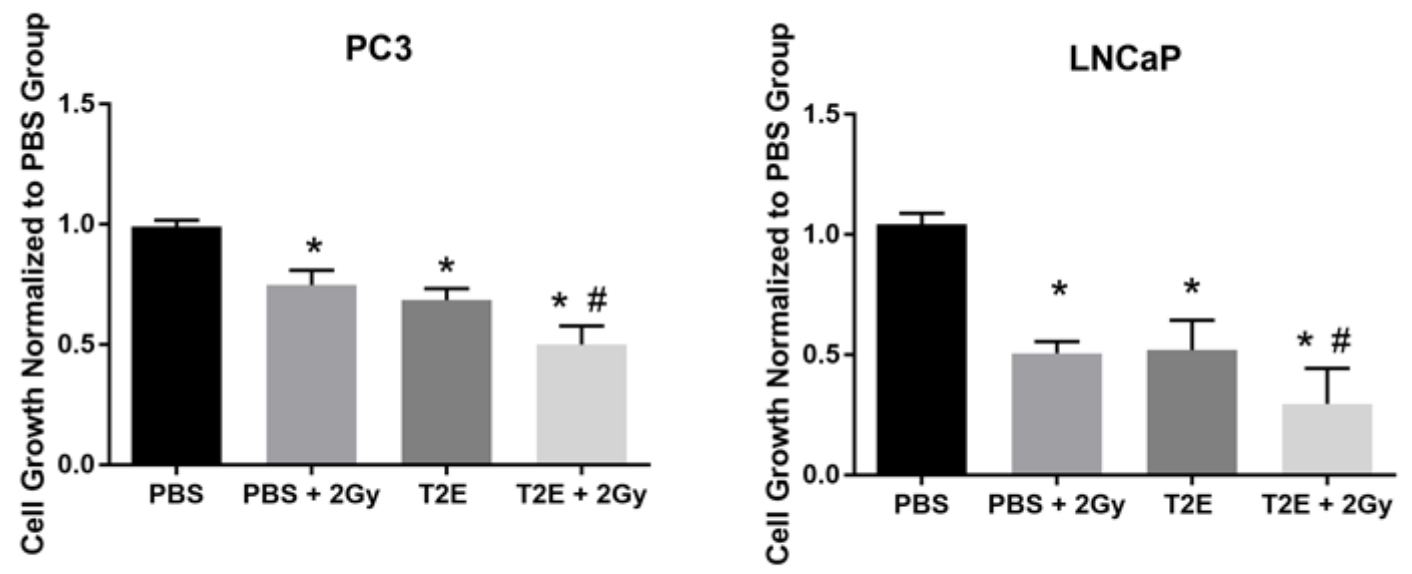


\subsection{In vitro normal prostate cell proliferation with MnTE-2-PyP treatment}

To determine whether MnTE-2-PyP has effects on normal prostate cells, we treated the human prostate fibroblasts cells, HPrF, with MnTE-2-PyP. The cell growth was compared between PBS and MnTE-2-PyP treatment after 96 hours. Interestingly, although MnTE-2-PyP did not significantly reduce HPrF cell proliferation (Figure 5), there is a trend of suppression $(p=0.074)$. 
Figure 5: HPrF cells growth with PBS or MnTE-2-PyP treatment. HPrF cells were treated with PBS or $30 \mu \mathrm{M}$ MnTE-2-PyP, cell numbers were counted after 96 hours of treatment. Data represent mean \pm SD from at least three independent experiments. 


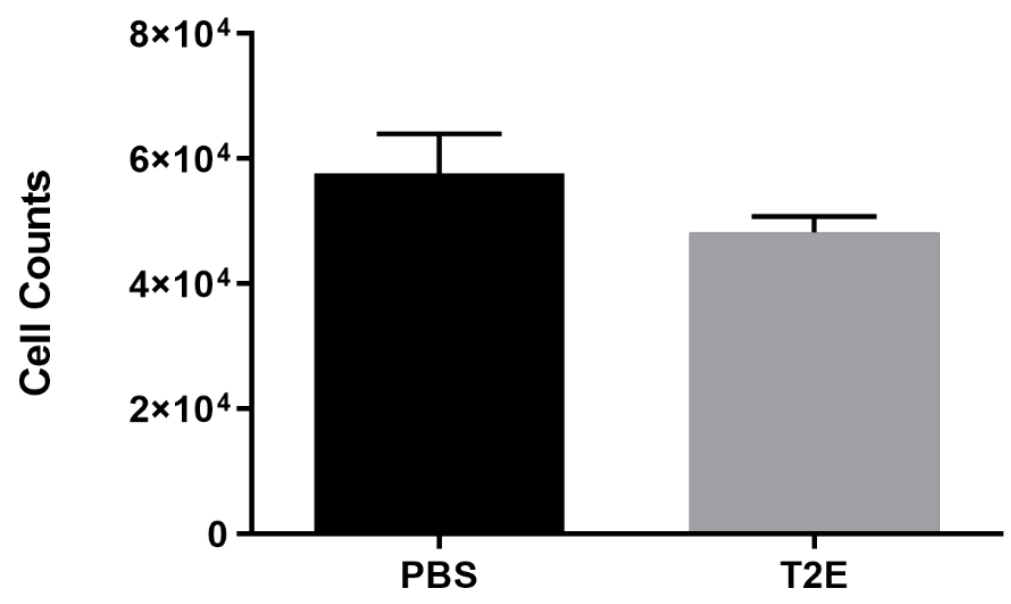




\subsection{Intracellular $\mathrm{H}_{2} \mathrm{O}_{2}$ measurements in prostate cancer cells with MnTE-2- PyP treatment}

The SOD mimetic, MnTE-2-PyP, scavenges superoxide into $\mathrm{H}_{2} \mathrm{O}_{2}$. To determine whether the $\mathrm{H}_{2} \mathrm{O}_{2}$ increase is associated with MnTE-2-PyP-induced cell growth inhibition, we first measured intracellular $\mathrm{H}_{2} \mathrm{O}_{2}$ levels in multiple prostate cancer cell lines with MnTE2-PyP and/or radiation treatment. Two different assays/probes, ROS-Glo and Peroxy orange 1 (PO1), were used. Based on the ROS-Glo assay, we found that in all prostate cancer cell lines, MnTE-2-PyP treatment increased intracellular $\mathrm{H}_{2} \mathrm{O}_{2}$ by $50 \sim 100 \%$ as compared to PBS treatment; however, $\mathrm{H}_{2} \mathrm{O}_{2}$ levels were not further enhanced by MnTE2-PyP with radiation. In addition, there is a major difference in basal $\mathrm{H}_{2} \mathrm{O}_{2}$ levels among these prostate cancer cells: LNCaP cells had the lowest detectable $\mathrm{H}_{2} \mathrm{O}_{2}$ levels among all of prostate cancer cell lines (Figure 6). We also used the PO1 probe to validate MnTE-2PyP's effects in PC3 and LNCaP cells, and MnTnBuOE-2-PyP (another SOD mimic) was

also used. Consistent with what we observed in the ROS-Glo assay, MnTE-2-PyP and MnTnBuOE-2-PyP significantly increased the $\mathrm{H}_{2} \mathrm{O}_{2}$ levels in PC3 cells, without further increase when radiation was combined; however, we did not detect any increase in LNCaP cells. This may be due to the low basal $\mathrm{H}_{2} \mathrm{O}_{2}$ levels in $\mathrm{LNCaP}$ cells that is below the sensitivity of the PO1 probe (Figure 7). 
Figure 6: $\mathrm{H}_{2} \mathrm{O}_{2}$ measurements in prostate cancer cells with MnTE-2-PyP with radiation treatment using the ROS-Glo probe. Intracellular $\mathrm{H}_{2} \mathrm{O}_{2}$ levels were measured in multiple prostate cancer cell lines with PBS or MnTE-2-PyP \pm 2 Gy radiation after 24 hours treatment. Data represent mean \pm SD from at least three independent experiments. ${ }^{*} p<0.05$ compared to PBS treatment in each cell line. 


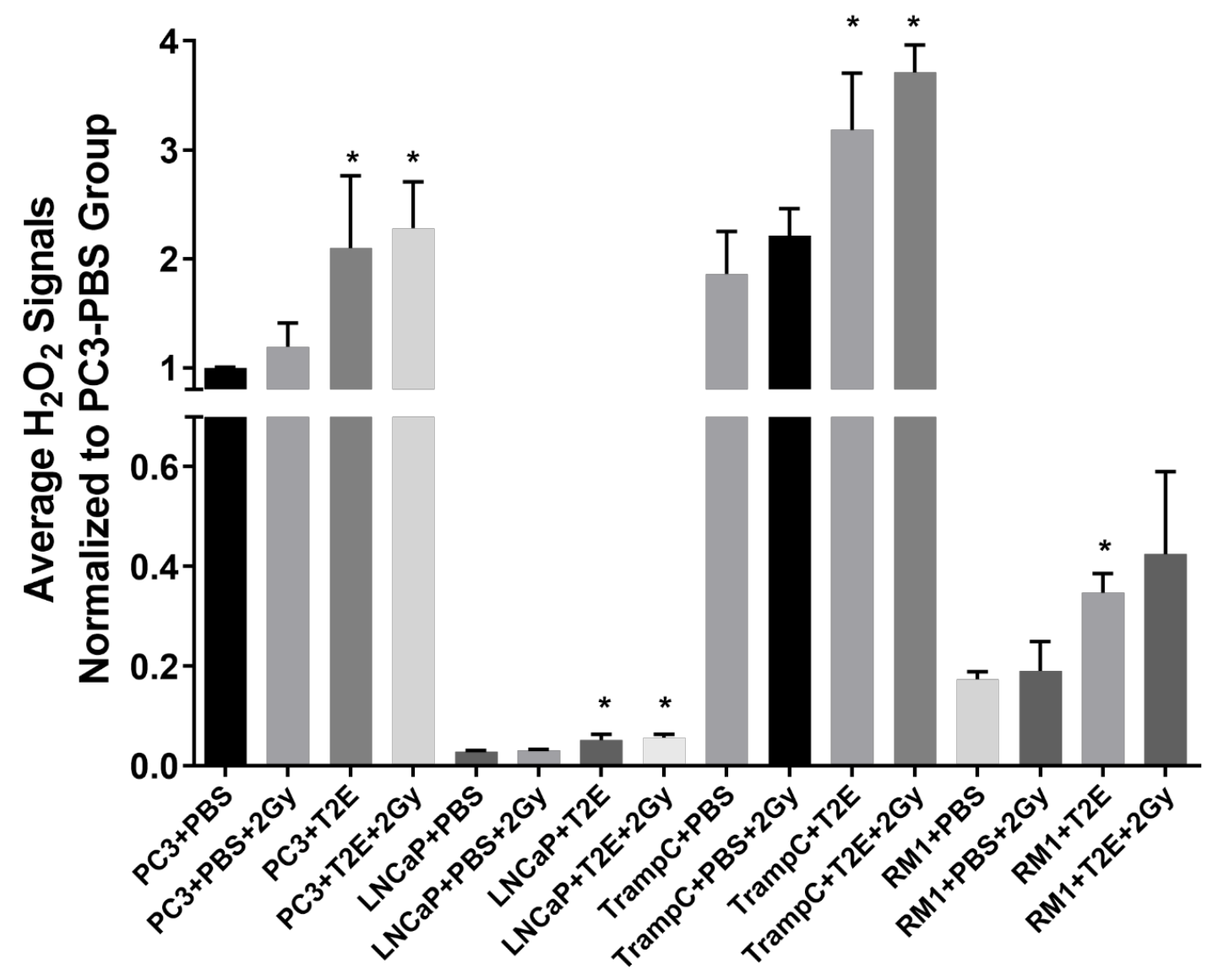


Figure 7: $\mathrm{H}_{2} \mathrm{O}_{2}$ measurements in prostate cancer cells with MnTE-2-PyP/MnTnBuOE2-PyP and radiation treatment using the P01 probe. (A) Representative images of LNCaP (left panel) and PC3 (right panel) cells treated with either PBS, MnTE-2-PyP (30 $\mu \mathrm{M})$, or MnTnBuOE-2-PyP $(0.5 \mu \mathrm{M}) \pm$ radiation (2 Gy) (B) Quantification of the PO1 staining in LNCaP and PC3 cells treated with PBS, MnTE-2-PyP, or MnTnBuOE-2-PyP \pm radiation $(2 \mathrm{~Gy})$. Data represent mean $\pm \mathrm{SD}$ from at least three independent experiments. ${ }^{*} p<0.05$ compared to PBS treatment. 
A

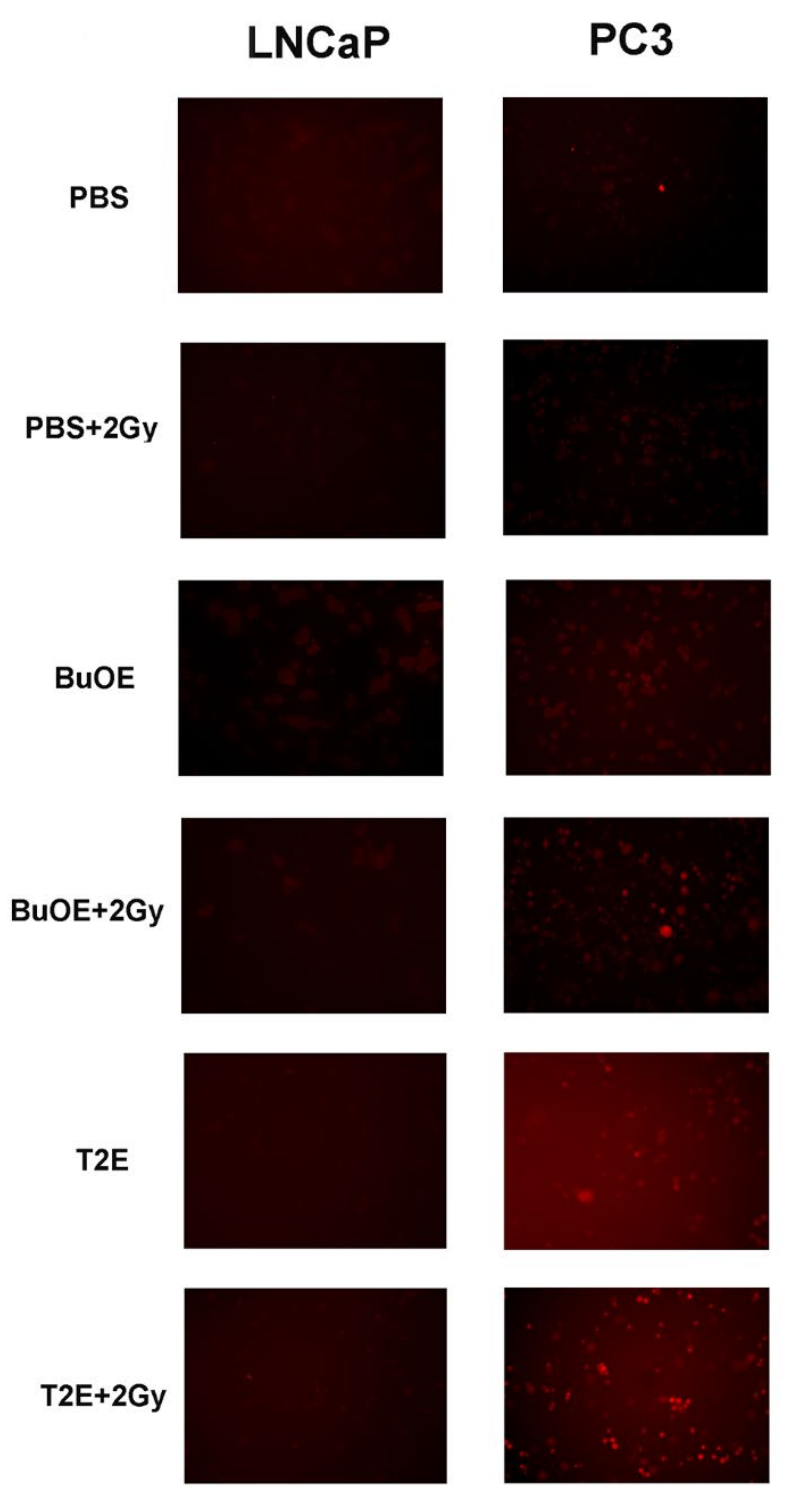


B
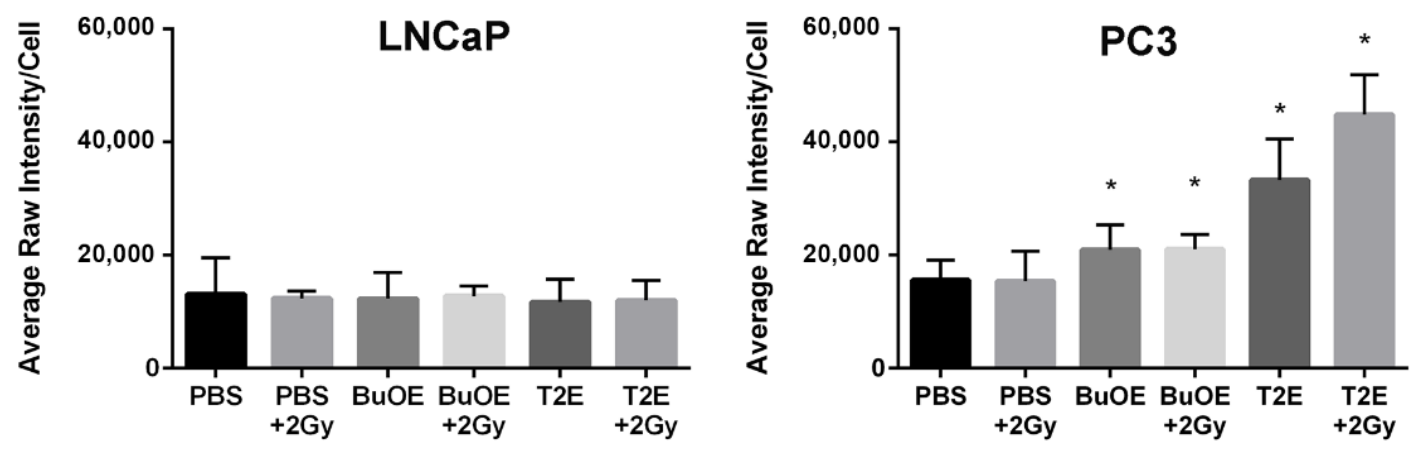


\subsection{Intracellular superoxide levels measurement in normal prostate cells and prostate cancer cells}

To identify whether superoxide levels play a role in MnTE-2-PyP-induced cancer cell growth inhibition, we first measured the basal levels of superoxide in both normal prostate cells and prostate cancer cells. The $\mathrm{CMH}$ spin probe can react with several radicals including the superoxide, peroxyl radical, peroxynitrite and nitrogen dioxide and produce the $\mathrm{CMH}$ radical that is detectable with electron paramagnetic resonance (EPR), however the $\mathrm{CMH}$ spin probe does not react with nitric oxide and $\mathrm{H}_{2} \mathrm{O}_{2}$ [147]. For total $\mathrm{CMH}$ radical signal, we did not find any difference among PC3, $\mathrm{LNCaP}$, and P3158 cells, and signals from these samples were not significantly different from the $\mathrm{CMH}$ alone control (Figure 8A). For CMH radical signal per cell, we did observe higher levels in P3158 cells as compared to prostate cancer cells (Figure 8B), yet this could be due to the fact that all test cells had similar background $\mathrm{CMH}$ radical signals while $\mathrm{P} 3158$ samples had fewer cell numbers calculated.

We also used MnTE-2-PyP with the CMH spin probe, but MnTE-2-PyP alone generated extremely high signals $\left(\mathrm{CMH}\right.$ control signal $=1.1 \times 10^{5}, \mathrm{CMH}+\mathrm{T} 2 \mathrm{E}$ signal $=$ $\left.8.6 \times 10^{6}\right)$, therefore, the $\mathrm{CMH}$ spin probe is not applicable to detect superoxide levels changes in MnTE-2-PyP-treated cells.

DHE is commonly used to detect overall ROS at $480 \mathrm{~nm}$ excitation wavelength. The $405 \mathrm{~nm}$ excitation wavelength, however, provides high specificity to $2 \mathrm{OH}-\mathrm{E}$, which is the end product of DHE after reacting with superoxide [148]. We found that radiation significantly enhanced superoxide signals in both PC3 and LNCaP cells, but MnPs treatment did not alter the levels. In addition, PC3 cells had higher superoxide-positive cells as compared to LNCaP cells at basal condition, but radiation enhanced superoxide levels in LNCaP cells more than PC3 cells (Figure 9). These data indicate that PC3 cells 
may be more oxidatively stressed than LNCaP cells. Based on our lab's previous results, where MnPs scavenged superoxide in normal prostate fibroblasts [95], it seems that MnPs did not reduce superoxide levels during radiotherapy, which could be a potential mechanism of MnPs distinct effects in normal prostate cells vs. prostate cancer cells [96]. 
Figure 8: Basal superoxide measurement in normal prostate cells and prostate cancer cells using the $\mathrm{CMH}$ spin probe. (A) Total $\mathrm{CMH}$ radical signal in tested cell lines. Signals were normalized to $\mathrm{CMH}$ alone control sample. (B) $\mathrm{CMH}$ radical signal per cell. Signals were normalized to PC3 cells and compared among three cell lines. Data represent mean \pm SD from at least three independent experiments. ${ }^{*} p<0.05$ compared to PC3 cells. 
A

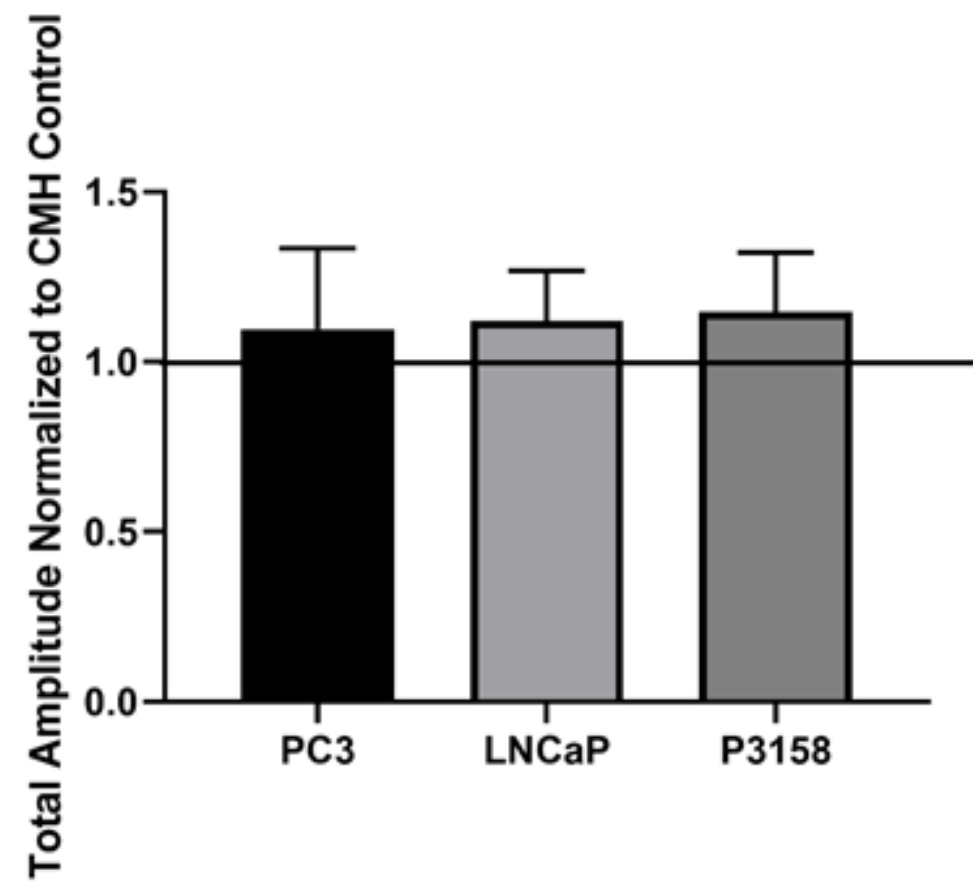

B

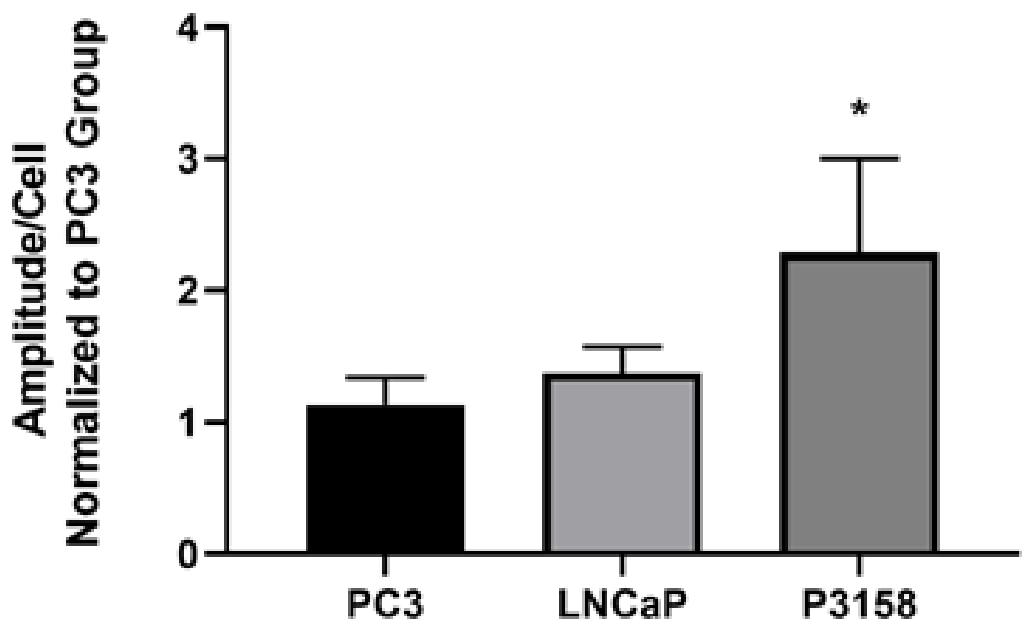


Figure 9: Superoxide levels in prostate cancer cells with MnPs \pm radiation measured with DHE. PC3 or LNCaP cells were treated with PBS, MnTE-2-PyP (30 $\mu \mathrm{M})$, or MnTnBuOE-2-PyP $(0.5 \mu \mathrm{M})$. In some cases, cells were irradiated (2 Gy) in the presence or absence of the respective MnPs. Cells were stained with DHE and subjected to flow cytometry analysis. The superoxide-specific wavelength (excitation $405 \mathrm{~nm} / \mathrm{emission} 570$ $\mathrm{nm}$ ) for the DHE probe was used. Data represent mean \pm SD from at least three independent experiments. ${ }^{*} p<0.05$ compared to PBS group. 

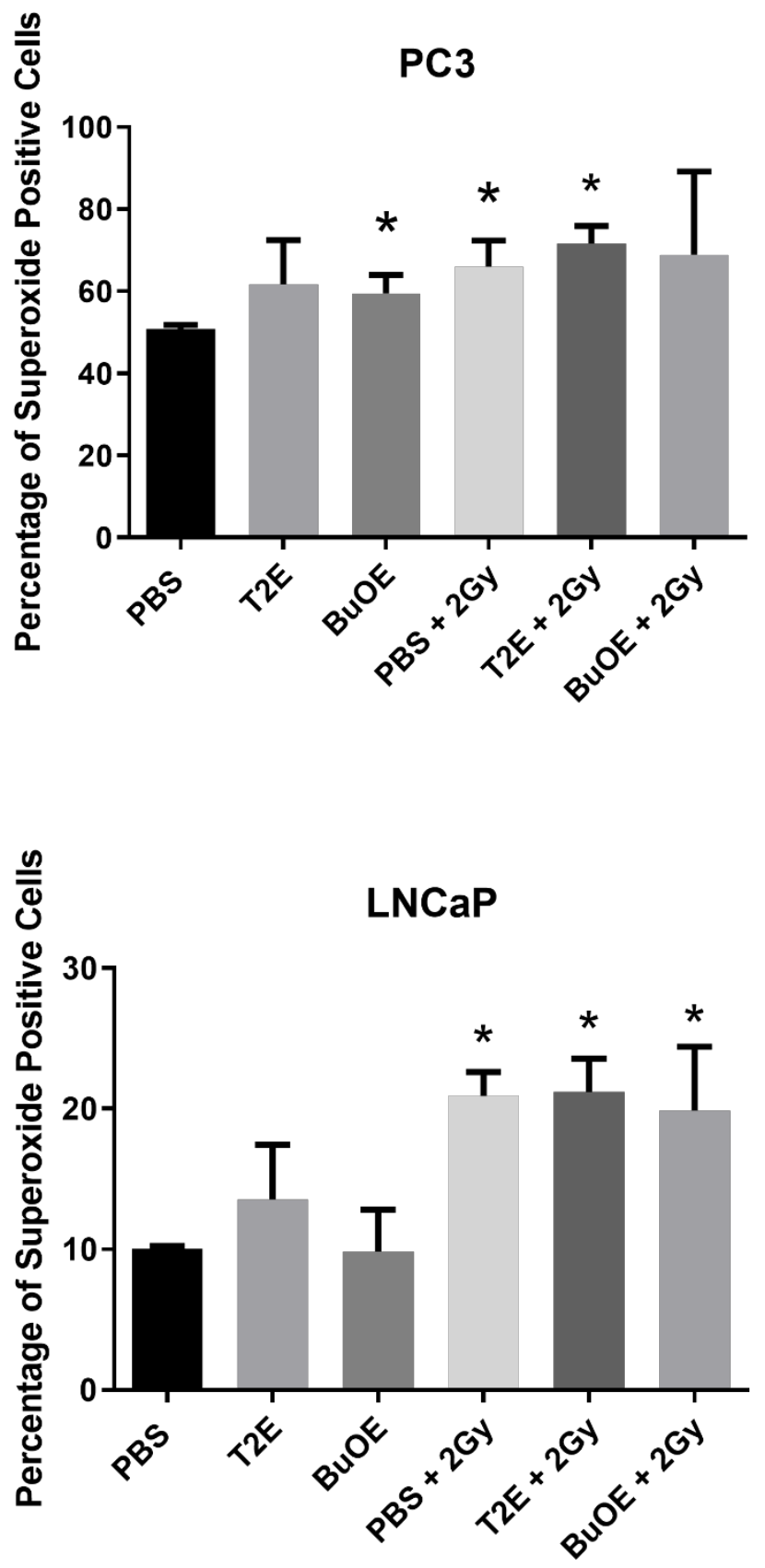


\subsection{Catalase activity in normal prostate cells and prostate cancer cells}

Catalase is one of the most important enzymes to scavenge $\mathrm{H}_{2} \mathrm{O}_{2}$, especially when cells face overwhelming $\mathrm{H}_{2} \mathrm{O}_{2}$ levels increase $[58,59]$. To determine whether catalase activity contributes to the different effects of MnTE-2-PyP in normal prostate cells and prostate cancer cells, we measured the catalase activity in multiple prostate cell lines with or without MnTE-2-PyP treatment. We found that LNCaP cells (LNCaP variants C81,C42B were also tested, data not shown) had significantly higher catalase activity than all other tested cells lines, and unexpectedly, the PMF cells had the lowest catalase activity. In addition, 48 hours of MnTE-2-PyP treatment did not alter the catalase activity in any tested cell line (Figure 10). These data indicate that the opposite response during radiotherapy of normal prostate cells and prostate cancer cells to MnTE-2-PyP is not caused by changes in catalase activity; however, the difference of catalase activity between PC3 and LNCaP cells may explain the different basal levels of $\mathrm{H}_{2} \mathrm{O}_{2}$. 
Figure 10: Comparison of catalase activity in normal prostate cells and prostate cancer cells with MnTE-2-PyP treatment. PC3, LNCaP, TrmapC, and PMF cells were treated with PBS or MnTE-2-PyP $(30 \mu \mathrm{M})$ for 48 hours and the catalase activity gels were run, for every single experiment, a PC3 cell sample was included as an internal control. (A) A representative image of catalase activity in all tested cells with PBS or MnTE-2-PyP treatment. (B) Densitometry analysis of catalase activity gels, catalase activity from each condition of every cell line was normalized to PBS-PC3. All data represent mean \pm SD from at least three independent experiments. ${ }^{*} p<0.05$ compared to PBS-PC3 group. 

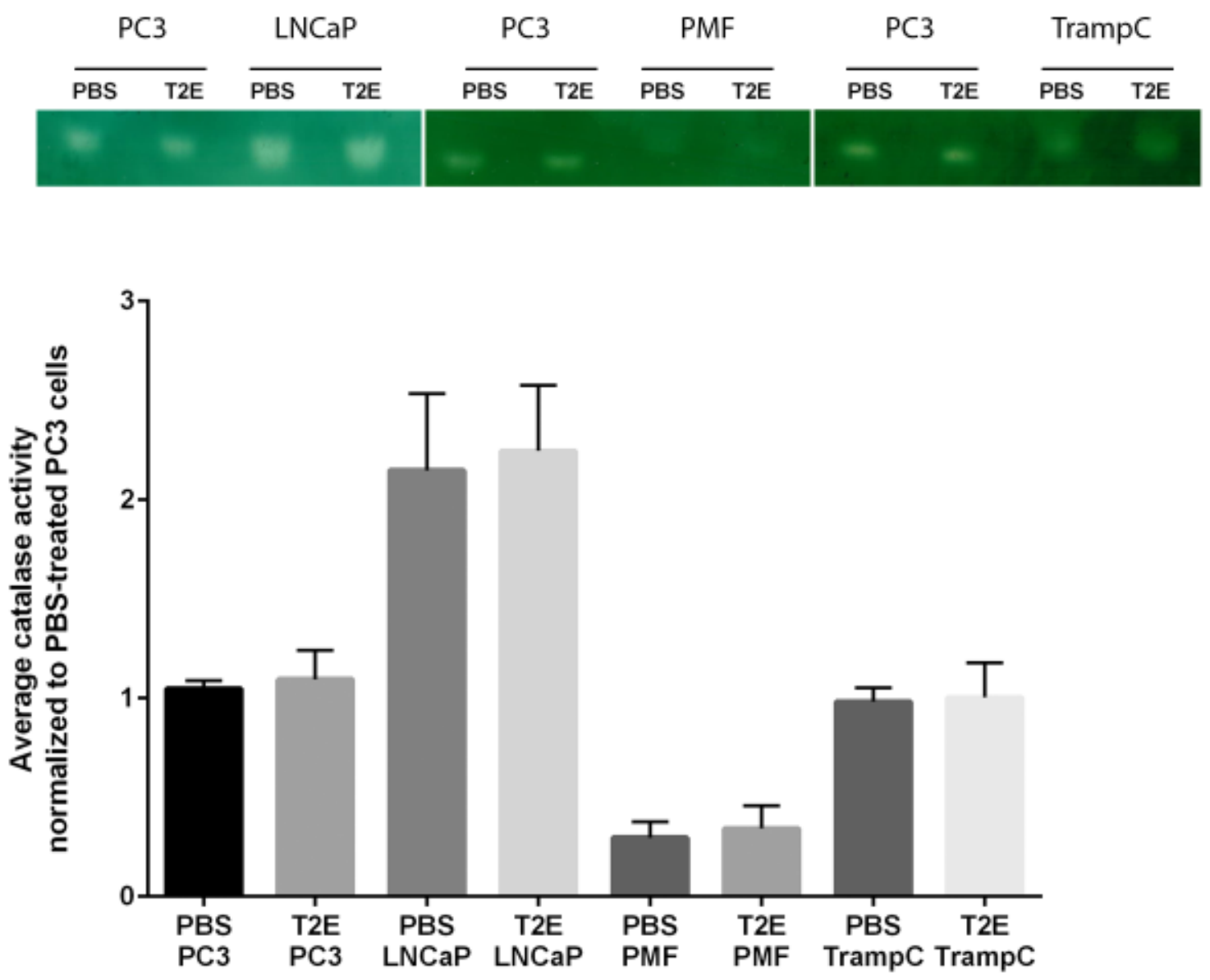


\subsection{Intracellular $\mathrm{H}_{2} \mathrm{O}_{2}$ levels alterations affect prostate cancer cell growth}

$\mathrm{H}_{2} \mathrm{O}_{2}$ can inhibit cell proliferation through redox signaling and oxidative damage. To determine whether the MnTE-2-PyP-induced $\mathrm{H}_{2} \mathrm{O}_{2}$ increase contributes to prostate cancer cell growth arrest, catalase adenovirus transduction and PEG-catalase were used in MnTE-2-PyP-treated PC3 cells. PC3 cells with a $50 \mathrm{MOI}$ transduction of catalase adenovirus produced a significant increase in cellular catalase activity as compared to control cells (Figure 11A-C); however, catalase transduction alone (10 or $50 \mathrm{MOI})$ caused around $20 \%$ growth inhibition in PC3 cells (Figure 11D). PC3 cells treated with MnTE-2PyP and catalase transduction (50 MOI) had similar $\mathrm{H}_{2} \mathrm{O}_{2}$ levels as compared to PBS control (Figure 11E). Correspondingly, catalase transduction rescued the growth arrest caused by MnTE-2-PyP treatment and these cells had a higher rate of proliferation as compared to control or empty vector-treated cells (Figure 11F, $p=0.0006 \& 0.028$ respectively). Previous studies have shown that both intracellular and extracellular $\mathrm{H}_{2} \mathrm{O}_{2}$ levels can affect cell growth [149-151]. To identify the source responsible for the arrested PC3 cell growth, we compared the proliferation rates of PEG-catalase-treated cells with PBS or MnTE-2-PyP treatment. We found that neither 1 hour (data not shown) nor 24 hours pre-treatment of $1000 \mathrm{U} / \mathrm{ml}$ PEG-catalase could enhance cellular catalase activity (Figure 11G, H). As a positive control, pure PEG-catalase compound was loaded onto the gel to ensure its $\mathrm{H}_{2} \mathrm{O}_{2}$-scavenging activity. The PEG-catalase was unable to rescue MnTE2-PyP-treated PC3 cells from growth inhibition (Figure 11I). These results indicate that the intracellular $\mathrm{H}_{2} \mathrm{O}_{2}$ balance is critical for $\mathrm{PC} 3$ cell growth. Too much $\mathrm{H}_{2} \mathrm{O}_{2}$, as was produced by MnTE-2-PyP treatment, resulted in reduced cell proliferation; too little $\mathrm{H}_{2} \mathrm{O}_{2}$, achieved through catalase transduction, also led to reduced proliferation.

Similar experiments were performed in LNCaP cells to determine the effects of $\mathrm{H}_{2} \mathrm{O}_{2}$ balance on cancer growth. As compared to PC3 cells, LNCaP cells were more 
sensitive to catalase adenovirus transduction, both 1 and $10 \mathrm{MOI}$ catalase adenovirus transduction significantly enhanced intracellular catalase activity (Figure 12A, B). Similarly, both 1 and $10 \mathrm{MOI}$ catalase adenovirus transduction alone could significantly induce LNCaP cell growth arrest, and $10 \mathrm{MOI}$ transduction greatly suppressed LNCaP cell proliferation (73.4\% decrease, Figure 12C, $p<0.0001)$. Correspondingly, $10 \mathrm{MOI}$ catalase transduction further decreased the $\mathrm{H}_{2} \mathrm{O}_{2}$ levels in both PBS and MnTE-2-PyP-treated cells to a lower extent than PBS control (Figure 12D), and MnTE-2-PyP could no longer inhibit cell proliferation (Figure 12E, right panel). Although $1 \mathrm{MOI}$ enhanced catalase activity, this level of transduction did not significantly decrease $\mathrm{H}_{2} \mathrm{O}_{2}$ levels (Figure 12D, $p=0.080$ ) and could not rescue the MnTE-2-PyP-induced growth arrest (Figure 12E right panel, $p<$ 0.0001). Therefore, both PC3 and LNCaP cells are sensitive to $\mathrm{H}_{2} \mathrm{O}_{2}$ level changes, either increase or decrease of $\mathrm{H}_{2} \mathrm{O}_{2}$ levels could alter cell proliferation; however, these two prostate cancer cell lines show different sensitivity to $\mathrm{H}_{2} \mathrm{O}_{2}$ levels. These data indicate that one of the main mechanisms by which MnTE-2-PyP inhibits prostate cancer growth is through the production of $\mathrm{H}_{2} \mathrm{O}_{2}$. 
Figure 11. MnTE-2-PyP suppressed PC3 cells growth through intracellular $\mathrm{H}_{2} \mathrm{O}_{2}$ levels increase. (A) A representative image of catalase activity in PC3 cells with different MOI catalase adenovirus (CAT)/empty vector (Empty) transduction. (B)\&(C) Densitometry analysis of the in gel activity assay shown in (A). (D) Relative PC3 cell counts with adenovirus transduction after 96 hours. (E) Measurement of cellular $\mathrm{H}_{2} \mathrm{O}_{2}$ levels and (F) relative PC3 cell counts with $50 \mathrm{MOI}$ catalase adenovirus/empty vector transduced after 96 hours treatment of PBS or MnTE-2-PyP. (G) Cellular catalase activity of PC3 cells treated with PEG-catalase (1000 U/ml) or PEG (corresponding amount of $1000 \mathrm{U} / \mathrm{ml}$ PEGcatalase) for 24 hours. (H) Densitometry analysis of (G). (I) Relative cell counts of PC3 cells after 96 hours treatment of PBS or MnTE-2-PyP with PEG or PEG-catalase. All data represent mean \pm SD from at least three independent experiments. ${ }^{*} p<0.05$ compared to PBS treatment. 
A

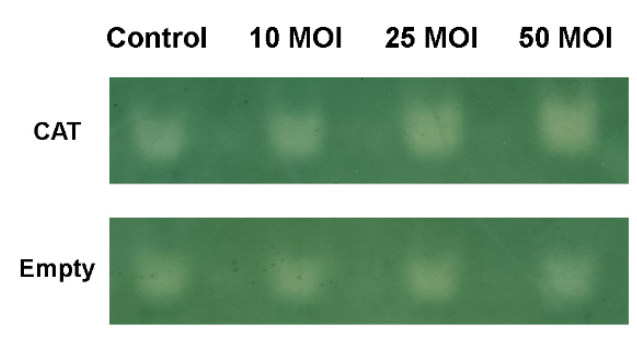

C

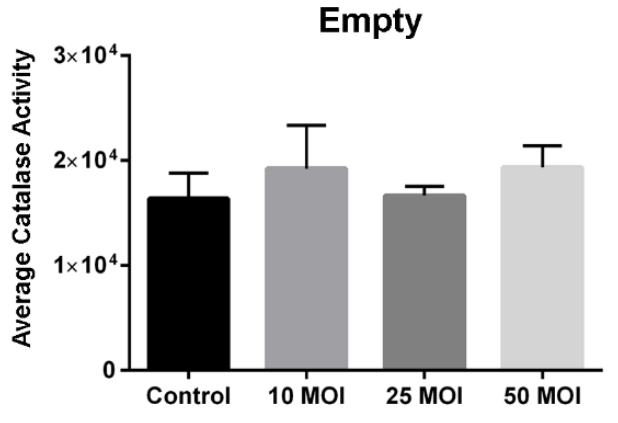

E

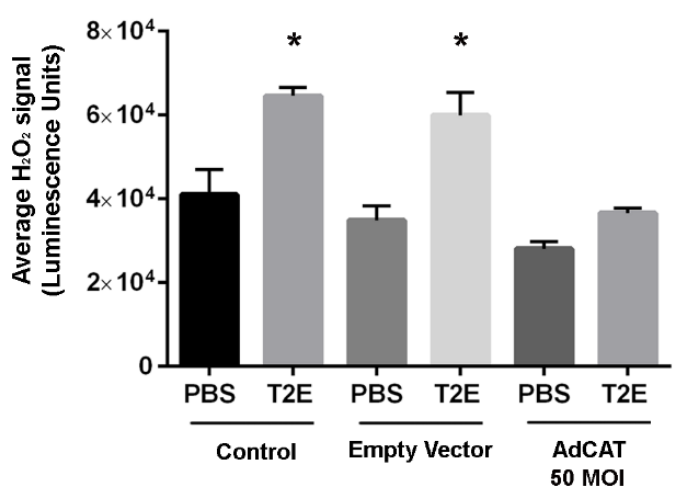

B

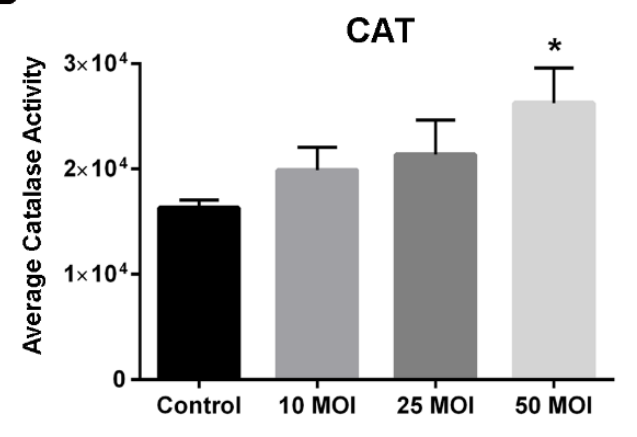

D

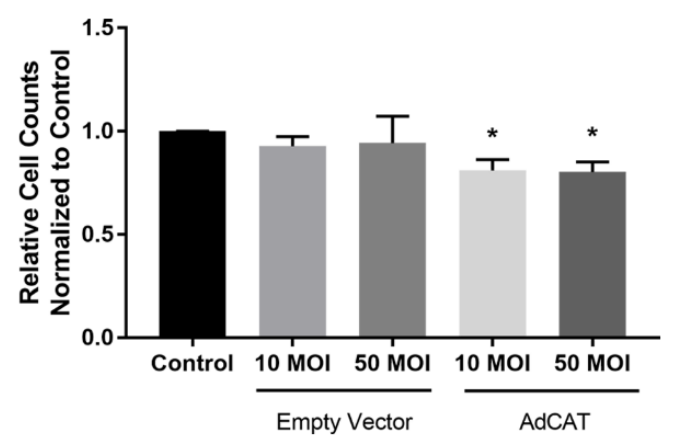

F

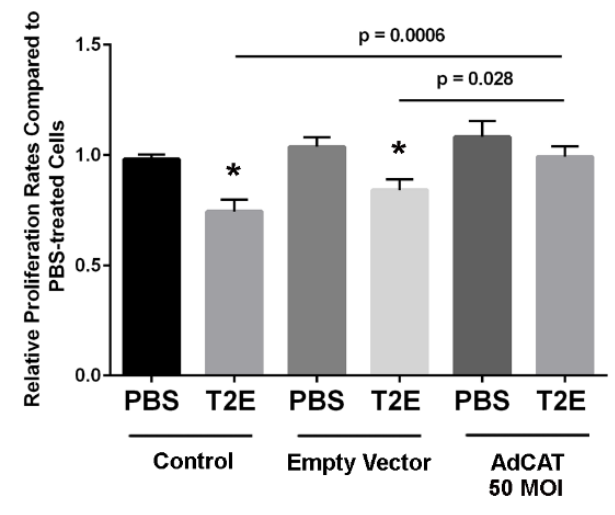




\section{G}

Negative $1000 \mathrm{U} / \mathrm{ml} 1000 \mathrm{U} / \mathrm{ml}$ Positive

Control PEG PEG-CAT Control
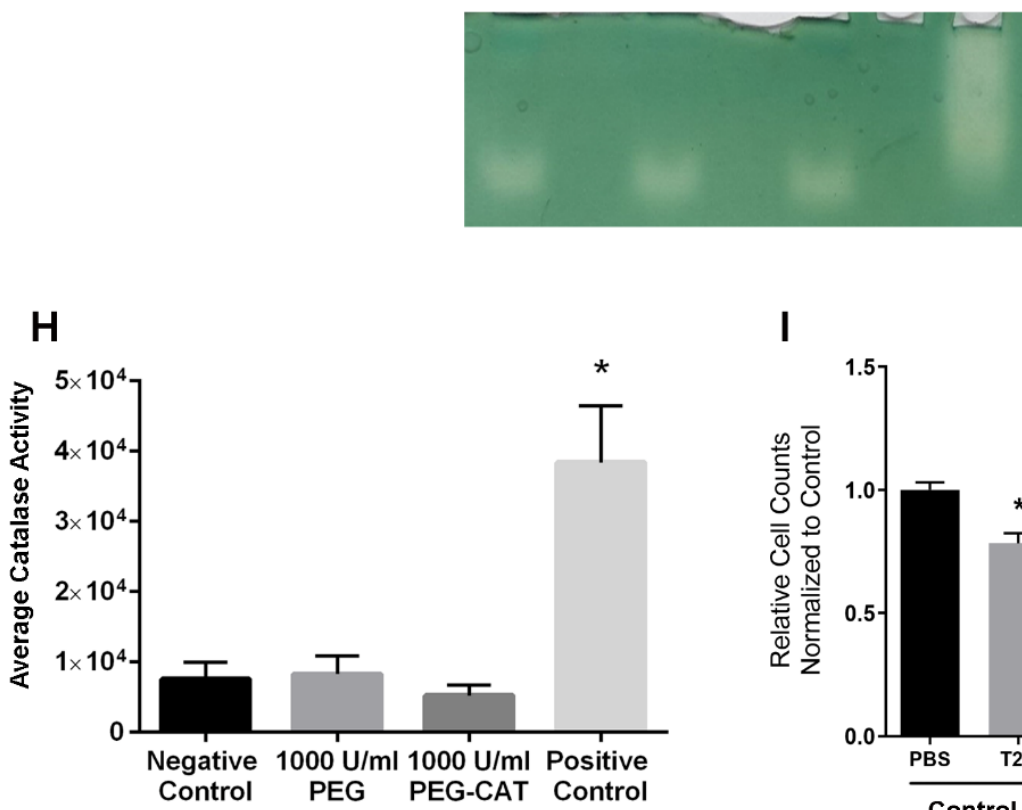

I

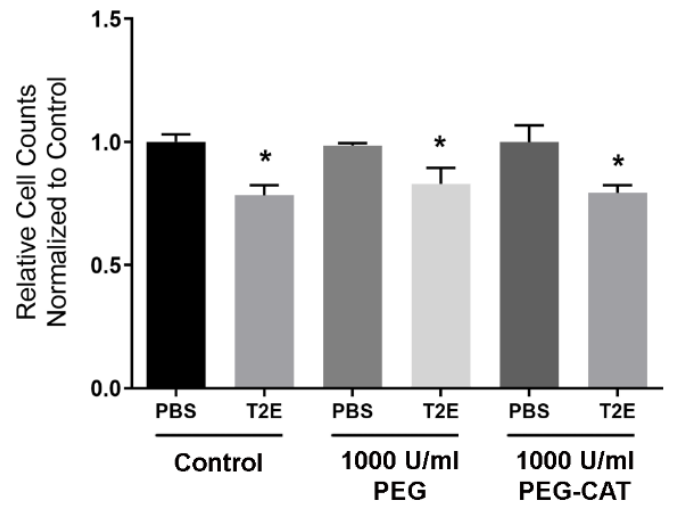


Figure 12. MnTE-2-PyP suppressed LNCaP cells growth through intracellular $\mathrm{H}_{2} \mathrm{O}_{2}$ levels increase. (A) A representative image of catalase activity in LNCaP cells with different MOI catalase adenovirus/empty vector transduction. (B) Densitometry analysis of the in gel activity assay shown in (A). (C) LNCaP cell growth with adenovirus transduction after 96 hours. (D) Measurement of cellular $\mathrm{H}_{2} \mathrm{O}_{2}$ levels and (E) cell growth of LNCaP cells with $50 \mathrm{MOI}$ catalase adenovirus/empty vector transduced after 96 hours treatment of PBS or MnTE-2-PyP. All data represent mean \pm SD from at least three independent experiments. ${ }^{*} p<0.05$ compared to PBS treatment. 


\section{A}

$$
\begin{array}{llll}
\text { Control } & \begin{array}{l}
\text { Empty } \\
\text { Vector }
\end{array} \text { MdCAT } & 10 \mathrm{MOI} \\
\text { AdCAT }
\end{array}
$$

B

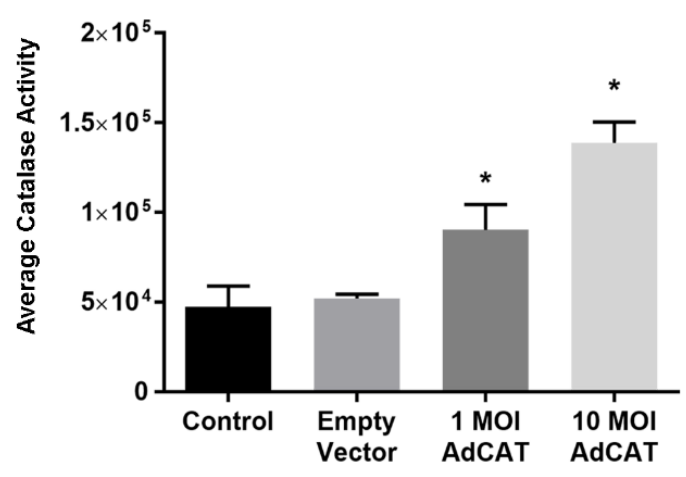

D

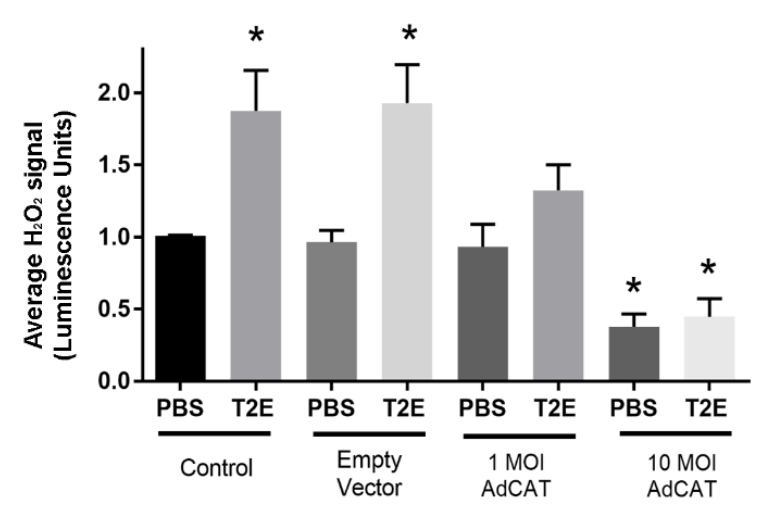

C

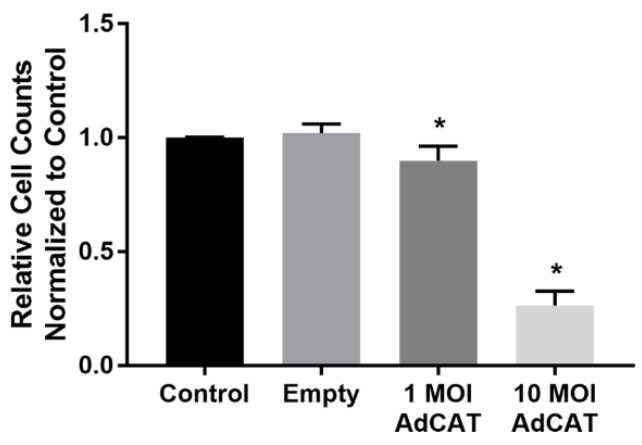

E

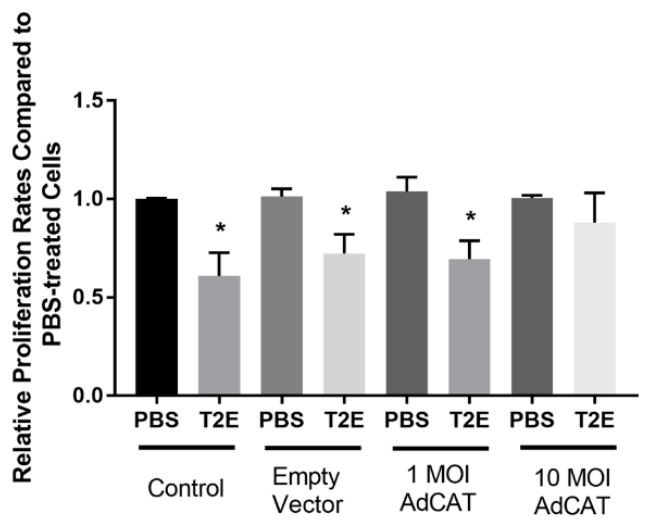




\subsection{Identifying protein oxidation in normal prostate cells and prostate cancer cells with MnTE-2-PyP treatment (BIAM assay)}

To determine whether MnTE-2-PyP induced $\mathrm{H}_{2} \mathrm{O}_{2}$ increase may lead to protein oxidation, we used the BIAM probe in multiple prostate cells lines that were treated with MnTE-2-PyP to identify the thiol oxidation states of proteins from whole cell lysates: PC3 is androgen-independent human prostate cancer cell line; LNCaP is androgen-dependent human prostate cancer cell line, and both C42B and C81 are derived from LNCaP cells; $\mathrm{HPrF}$ is a primary human prostate fibroblast cell; PMF is a primary mouse prostate fibroblast cell isolated from C57BL/6 mouse prostate.

We found that in all 6 cell/cell lines tested, only PC3 cells had a significant decrease in enriched reduced proteins, indicating MnTE-2-PyP may cause protein oxidation in PC3 cells (Figure 13A). There is a trend of oxidation by MnTE-2-PyP in LNCaP, C42B, C81, and HPrF, but the difference is not significant (Figure 13B-E). In addition, no further oxidation was detected in MnTE-2-PyP-treated PMF cells (Figure 13F).

To identify what proteins were oxidized in MnTE-2-PyP-treated PC3 cells, we performed mass spectrometry analysis on a commassie gel. Consistently, MnTE-2-PyP treatment led to a significant decrease of protein-BIAM binding in PC3 cells, indicating a decrease of proteins with reduced thiols (Figure 14). Multiple bands in PBS and MnTE-2PyP-treated samples with obvious differences were cut out of the gel and sent for mass spectrometry analysis. We found that Ser/Thr protein phosphatase 1 catalytic subunit (PP1CB, the only consistent target, $n=3$ ) was detected in PBS-treated cells but not in MnTE-2-PyP-treated cells indicating that PP1CB may be oxidized or its expression level was downregulated with MnTE-2-PyP treatment. PP1CB is one of the three most important catalytic subunits of PP1, and contains several thiol groups and one catalytic center that are highly sensitive to ROS. Oxidation of thiol groups or the $\mathrm{Mn}-\mathrm{Mn} / \mathrm{Mn}-\mathrm{Fe}$ 
metal center can result in activity loss $[152,153]$. We measured the PP1CB protein levels in PC3 and LNCaP cells. There was no difference of PP1CB levels between PC3 and LNCaP cells (Figure 15D), and the addition of MnTE-2-PyP did not alter PP1CB expression levels (Figure 15B, C). These data suggest that PP1CB was oxidized under MnTE-2-PyP treatment.

PP1 and PP2A contribute more than $90 \%$ of all cellular PPP activity and they are involved in various signaling pathways [154]. PP1 and PP2A can directly or indirectly control retinoblastoma protein $(\mathrm{pRB})$ phosphorylation, which is a key regulator of cell growth [154-157]. Under oxidative stress, PP2A is able to induce pRB dephosphorylation; while a loss of PP1 activity can dephosphorylate pRB through the PP1-cyclin D1-pRb pathway [158-160]. To determine whether MnTE-2-PyP treatment altered protein phosphatase activity, we measured total PPP activity and PP1 activity by using a PP2Aspecific inhibitor, okadaic acid. PP2A is often lost or inactivated in cancer through induction of expression of endogenous inhibitors of PP2A, such as CIP2A [161, 162]. We found that PP1 activity was significantly decreased in both PC3 and LNCaP cells with MnTE-2-PyP treatment (Figure 15E, G), but overall PPP activity did not change in the presence of MnTE-2-PyP (Figure 15F, H), which indicates that PP2A activity may increase to compensate for PP1 activity loss.

We also investigated whether increased $\mathrm{H}_{2} \mathrm{O}_{2}$ or thiol oxidation contributes to PP1 activity loss. Unexpectedly, neither $50 \mathrm{MOI}$ transduction of catalase adenovirus nor 1 hour treatment of 2 mM DTT rescued PP1 activity in MnTE-2-PyP-treated PC3 cells, indicating PP1 activity loss by MnTE-2-PyP treatment may not be due to $\mathrm{H}_{2} \mathrm{O}_{2}$ increase or thiol oxidation, the oxidation of metal center by MnTE-2-PyP could be an alternative explanation, thus, the reason for PP1 activity loss remians unknown (Figure 16). 
Figure 13: Determination of thiol oxidation in MnTE-2-PyP-treated normal prostate cells and prostate cancer cells. Proteins with reduced thiols from whole cell lysates were treated with or without MnTE-2-PyP $(30 \mu \mathrm{M})$ for 48 hours and enriched by BIAM probes and streptavidin agarose beads. As a control, the lysates were treated with the reducing agent DTT or the oxidizing agent diamide (DIA). (A) Left panel: a representative image of enriched reduced proteins in PC3 cells, right panel: densitometry analysis. (B) Left panel: a representative image of enriched reduced proteins in $\mathrm{LNCaP}$ cells, right panel: densitometry analysis. (C) Left panel: a representative image of enriched reduced proteins in C81 cells, right panel: densitometry analysis. (D) Left panel: a representative image of enriched reduced proteins in C42B cells, right panel: densitometry analysis. (E) Left panel: a representative image of enriched reduced proteins in HPrF cells, right panel: densitometry analysis. $(F)$ Left panel: a representative image of enriched reduced proteins in PMF cells, right panel: densitometry analysis. All data represent mean \pm SD from at least three independent experiments. ${ }^{*} p<0.05$ compared to PBS treatment. 
A

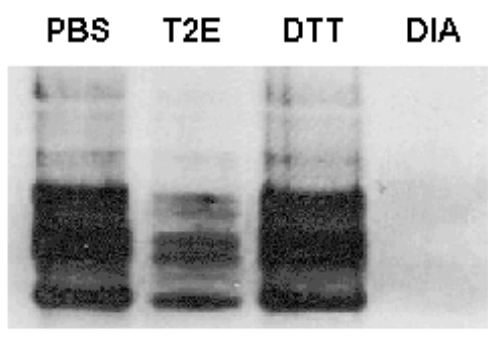

B

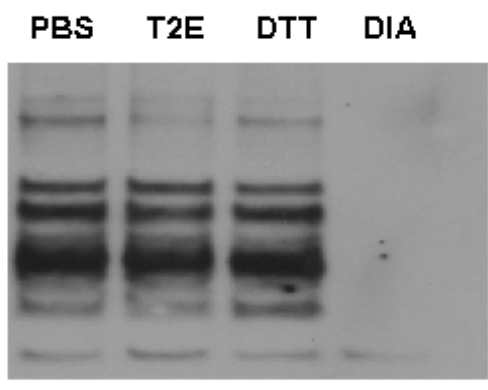

C

PBS T2E DTT DIA

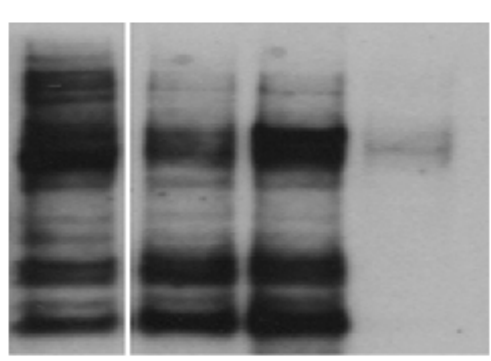

PC3

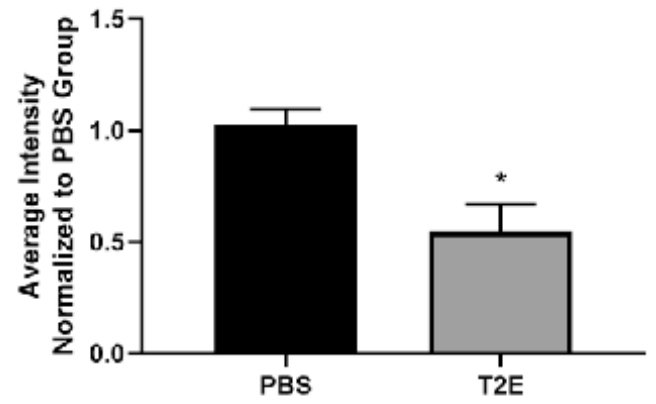

LNCaP

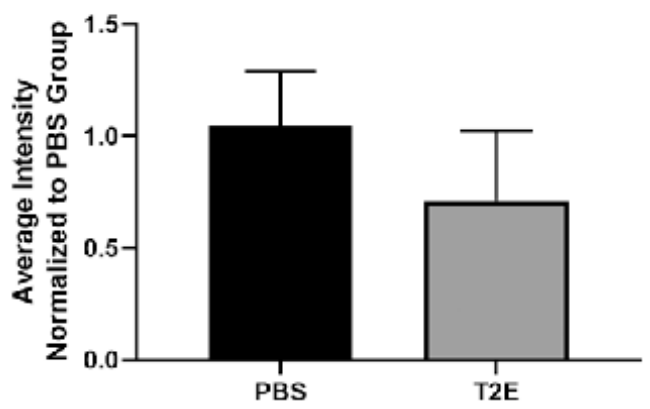

C81

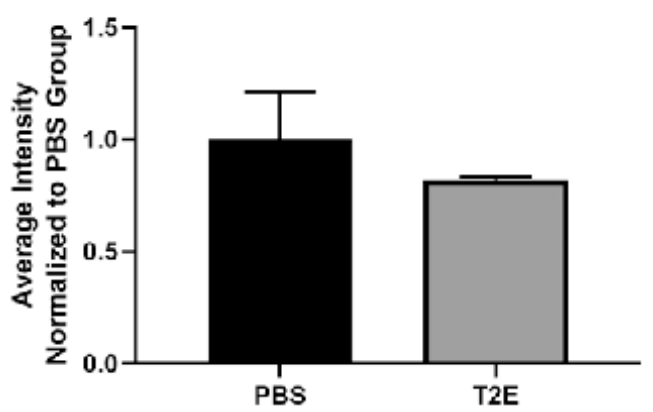


D

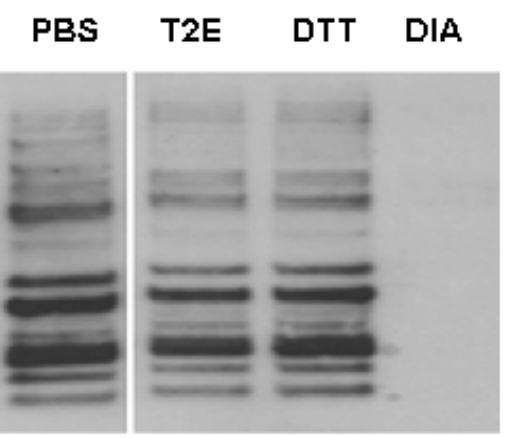

E

PBS T2E DTT DIA

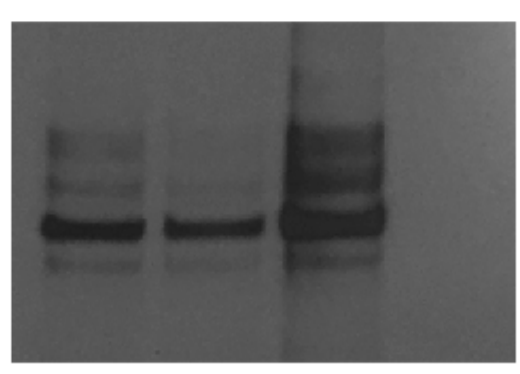

F

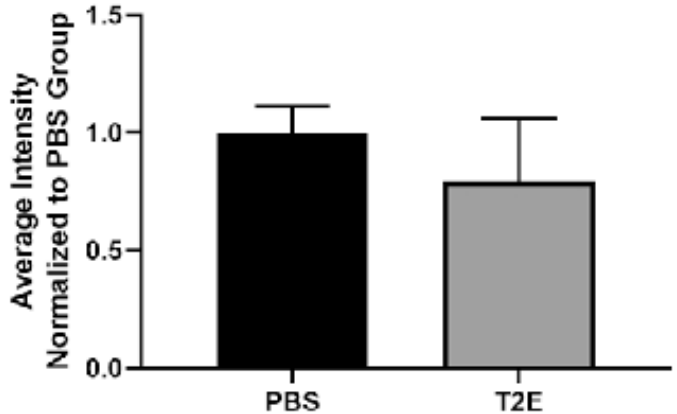

HPrF

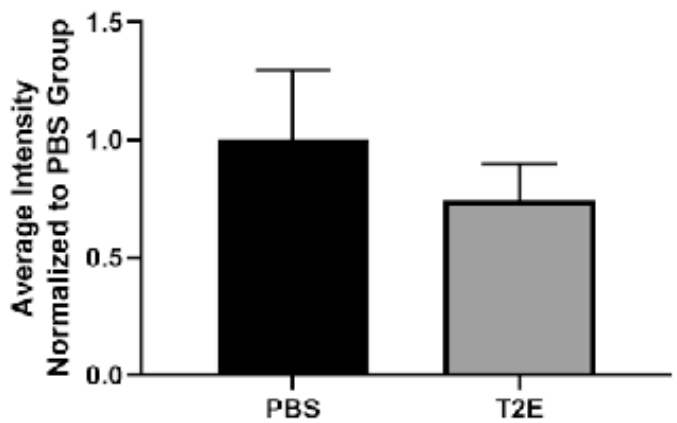

PMF

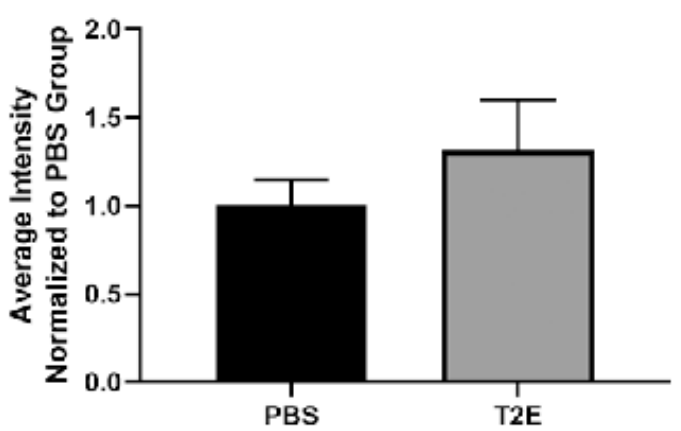


Figure 14: Identification of thiol oxidation in MnTE-2-PyP-treated PC3 cells. A representative image of Coomassie stained gels of non-oxidized proteins in PC3 cells. Proteins with reduced thiols were isolated by BIAM probe and selected regions of Coomassie gels (indicated by red boxes), where significant difference was observed, were cut and sent for mass spectrometry analyses. 


\section{PBS T2E}

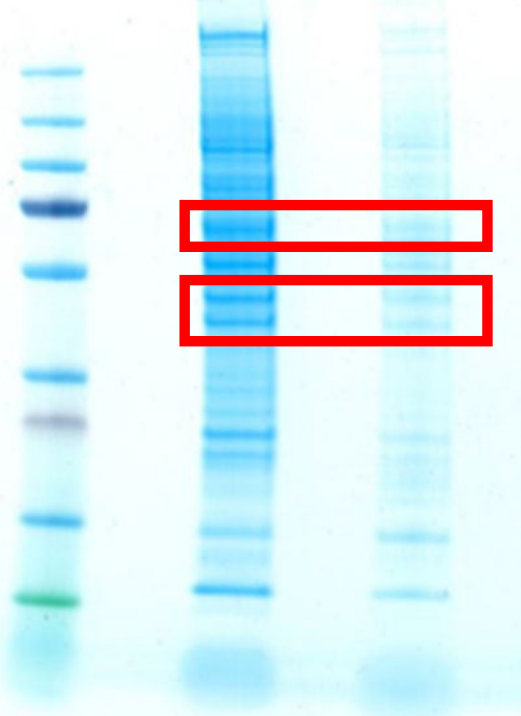


Figure 15. Identification of MnTE-2-PyP-induced PP1CB oxidation and PPPs activity measurement. (A) A representative image of a PP1CB western blot with Ponceau staining in PC3 cells and corresponding densitometry analysis. (B) A representative image of a PP1CB western blot with Ponceau staining in LNCaP cells and corresponding densitometry analysis. (C) A representative image of a PP1CB western blot with Ponceau staining in PC3 and LNCaP cells and corresponding densitometry analysis. (D) PP1 activity measurement in PC3 cells with PBS or MnTE-2-PyP treatment. (E) Total PPP activity measurement in PC3 cells with PBS or MnTE-2-PyP treatment. (F) PP1 activity measurement in LNCaP cells with PBS or MnTE-2-PyP treatment. (G) Total PPP activity measurement in LNCaP cells with PBS or MnTE-2-PyP treatment. All data represent mean \pm SD from at least three independent experiments. ${ }^{*} p<0.05$ compared to PBS treatment. 
A
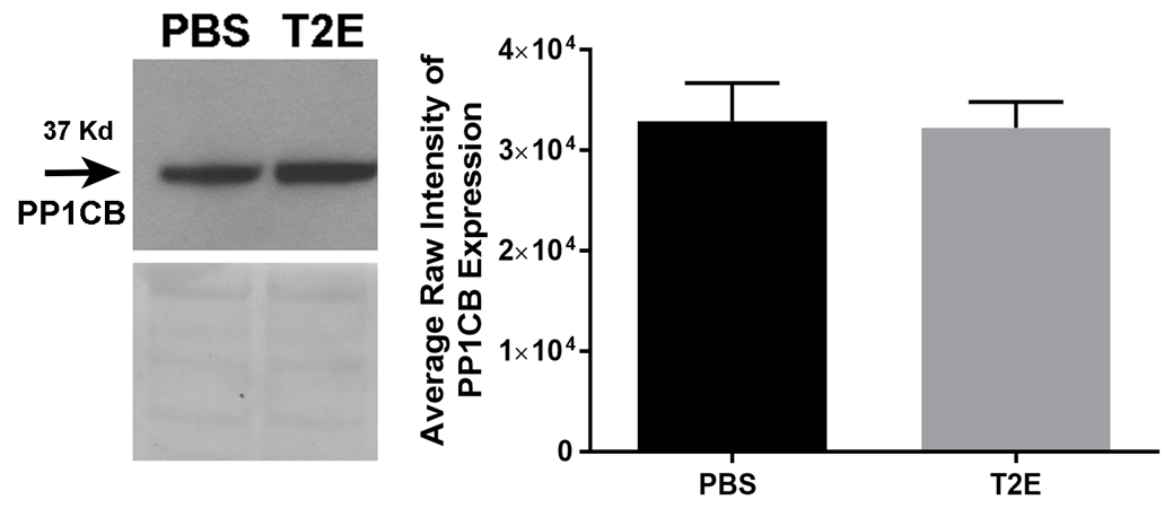

B
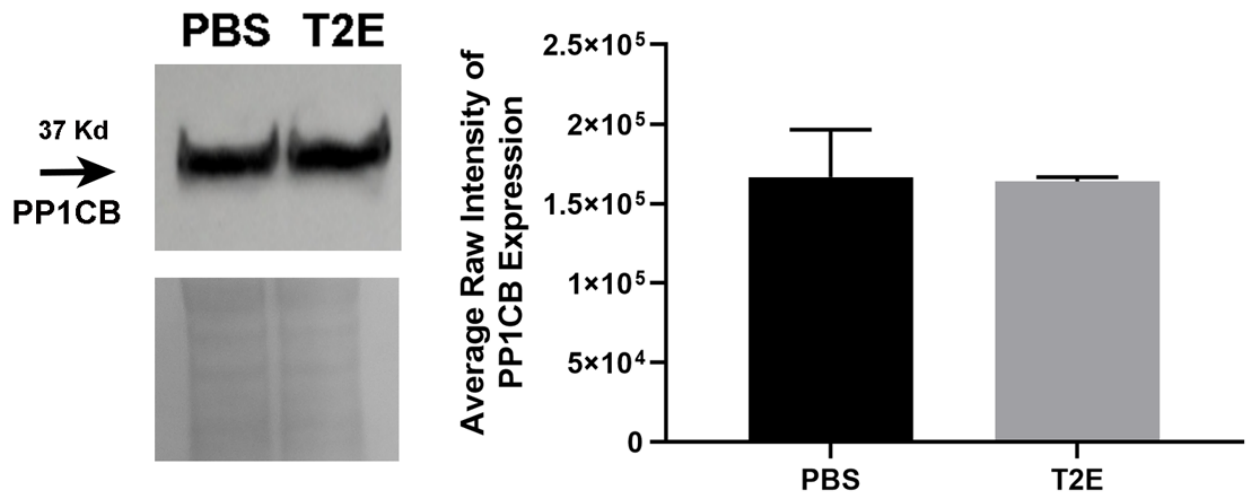

C
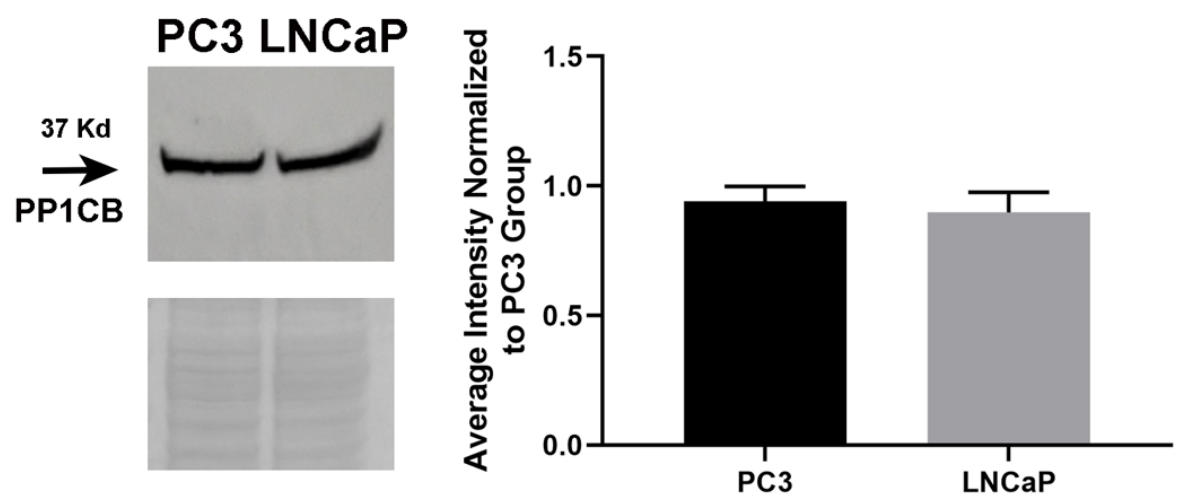
D

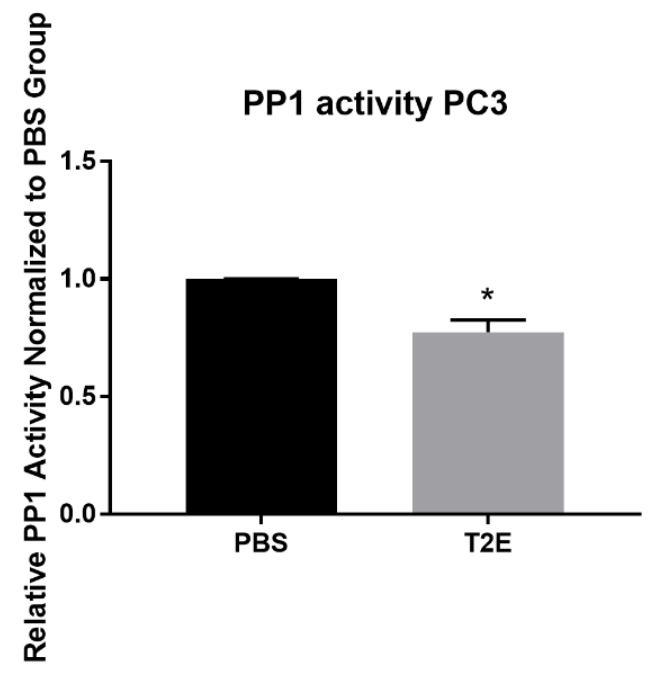

F

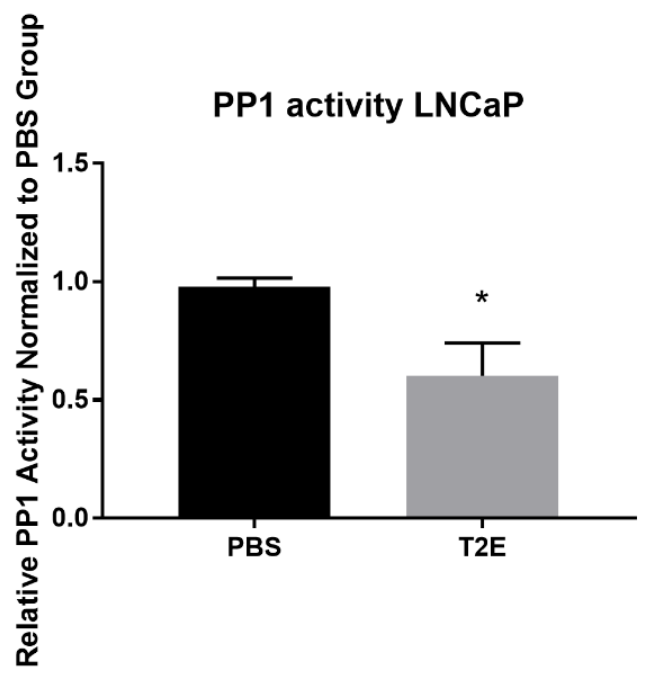

E

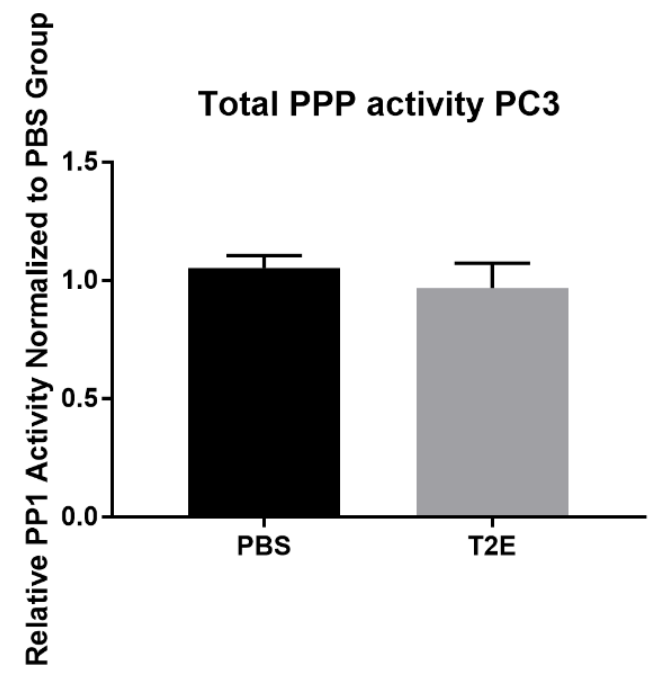

G

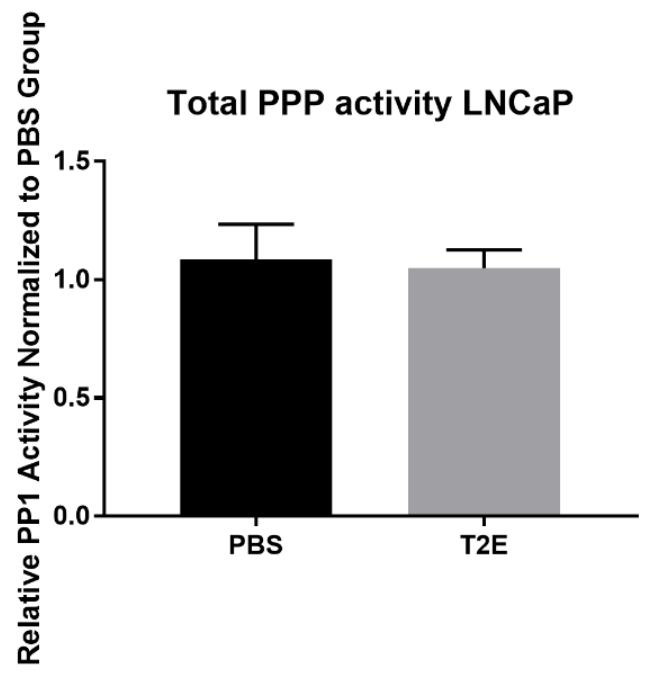


Figure 16. PP1 activity with catalase overexpression and DTT treatment in PC3 cells. PP1 activity measurement in PC3 cells with PBS or MnTE-2-PyP treatment, 50 MOI empty vector/catalase adenovirus and 1 hour treatment of $2 \mathrm{mM}$ DTT was applied to some conditions to reduce $\mathrm{H}_{2} \mathrm{O}_{2}$-mediated thiol oxidation. All data represent mean $\pm \mathrm{SD}$ from at least three independent experiments. ${ }^{*} p<0.05$ compared to PBS treatment. 


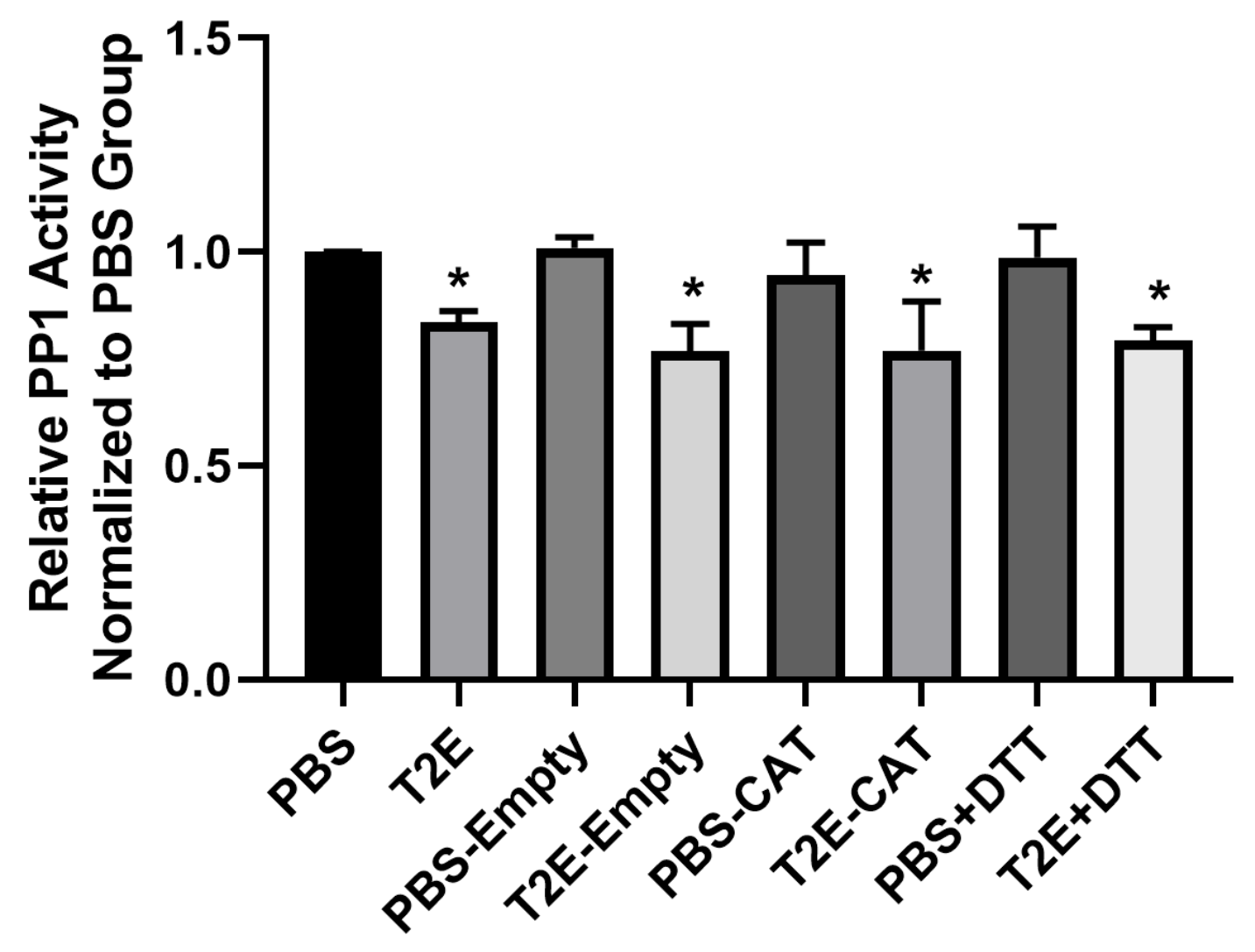




\subsection{MnTE-2-PyP affects PPP-regulated signaling pathways}

We measured several downstream targets regulated by PP1 and PP2A. MnTE-2PyP treatment enhanced cyclin D1 phosphorylation levels by $47 \%$ in PC3 cells as compared to PBS treatment (Figure 17A). Hyperphosphorylation (Thr 286) of cyclin D1 has been shown to suppress nuclear-cytosolic transportation and alter its stability [163, 164]. pRB, which is the target of cyclin D1 and suppresses excessive cell growth by inhibiting DNA replication, was hypophosphorylated in MnTE-2-PyP-treated PC3 cells (Figure 17B); however, in LNCaP cells, both cyclin D1 and pRB were hypophosphorylated (Figure 17C, D). This indicates that PP2A or other phosphatases may directly dephosphorylate pRB in LNCaP cells. We also compared pRB levels in both cell lines but no significant difference was observed (Figure 17E).

Several other proteins that potentially contribute to cyclin D1 hyperphosphorylation in PC3 cells were investigated. The expression levels of two cyclin-dependent kinase inhibitors, p16 and p21, were measured in both PBS- and MnTE-2-PyP-treated cells, but no substantial difference in the levels of protein expression were observed (Figure 17G-I) [165-167]. Therefore, PP1 appears to be the major regulator of cyclin D1 phosphorylation states in MnTE-2-PyP-treated PC3 cells. 
Figure 17. Phosphorylation levels and expression levels of PPP-regulated proteins. (A) Representative images of cyclin D1 and phospho-cyclin D1 (Thr 286) western blots and Ponceau staining in PC3 cells with densitometry of phosphorylation levels of cyclin D1. (B) Representative images of pRB and phospho-pRB (Ser-780) western blots and Ponceau staining in PC3 cells with densitometry of phosphorylation levels of pRB. (C) Representative images of cyclin D1 and phospho-cyclin D1 (Thr 286) western blots and Ponceau staining in LNCaP cells with densitometry of phosphorylation levels of cyclin D1. (D) Representative images of $\mathrm{pRB}$ and phospho-pRB (Ser-780) western blots and Ponceau staining in LNCaP cells with densitometry of phosphorylation levels of pRB. (E) Representative images of $\mathrm{pRB}$ western blots and Ponceau staining in both PC3 and LNCaP cells with densitometry of phosphorylation levels of pRB. (F)\&(G) Representative images of western blots for p16 and p21 in PC3 cells respectively with densitometry analysis of p16 and p21. $(H) \&(I)$ Representative images of western blots for p16 and p21 in LNCaP cells respectively with densitometry analysis of $p 16$ and $p 21$. All data represent mean \pm SD from at least three independent experiments. 
A
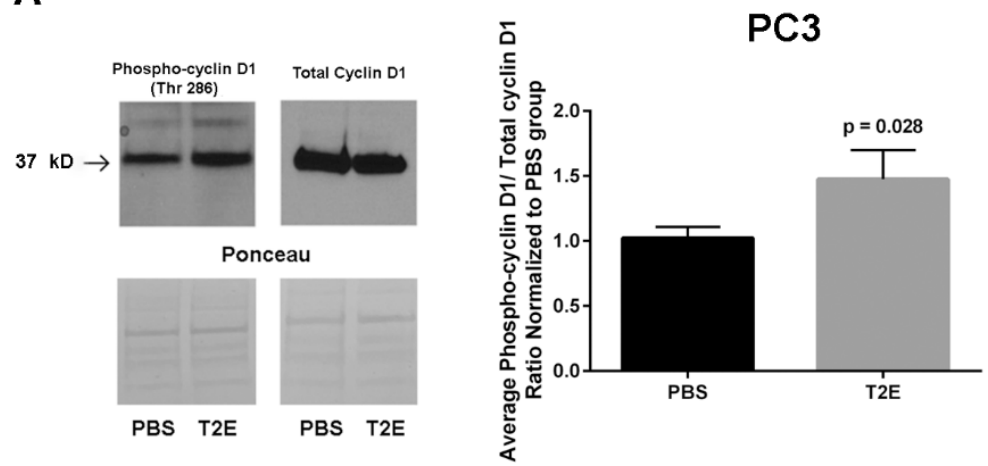

B
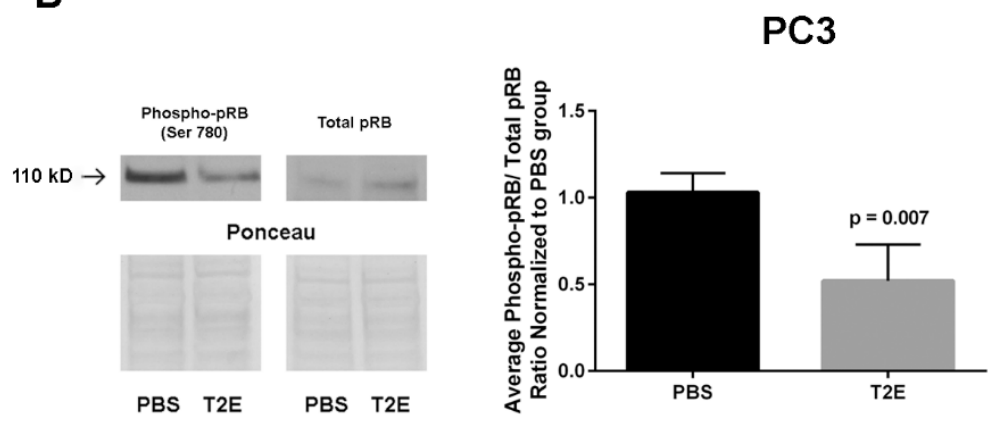

C
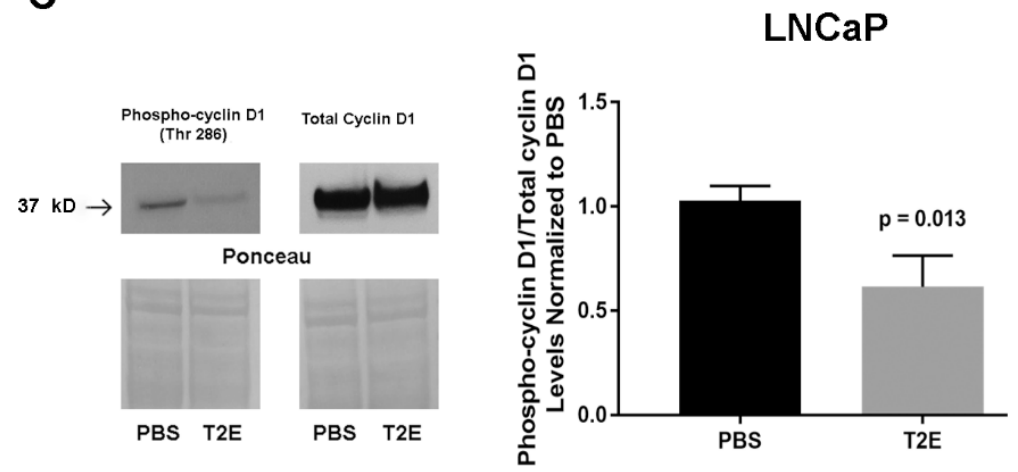

D

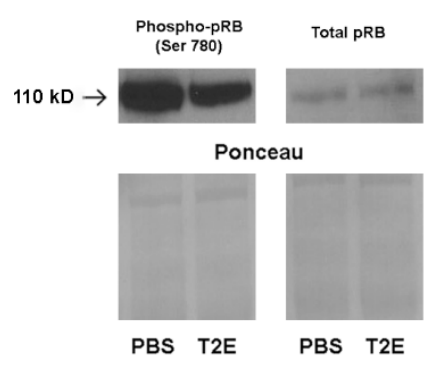

\section{LNCaP}

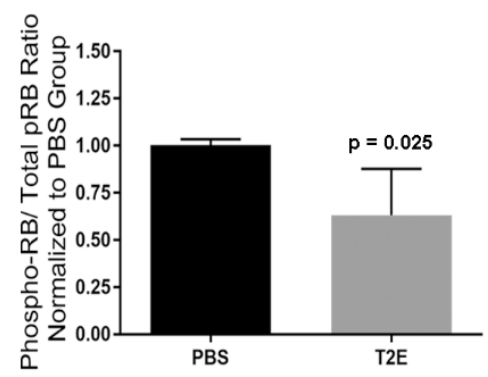



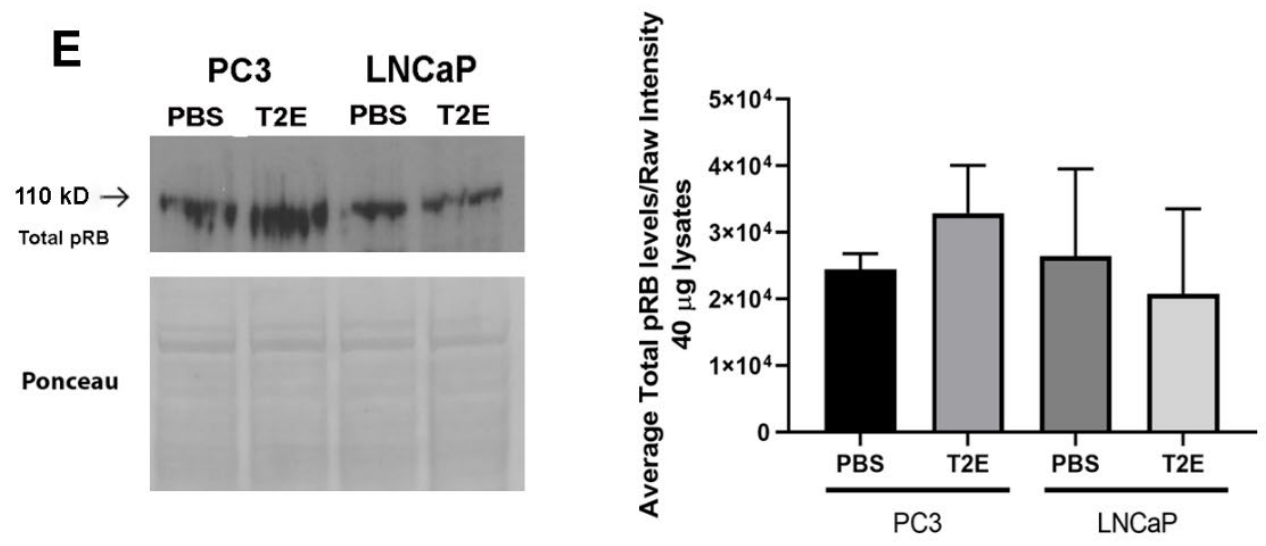

F
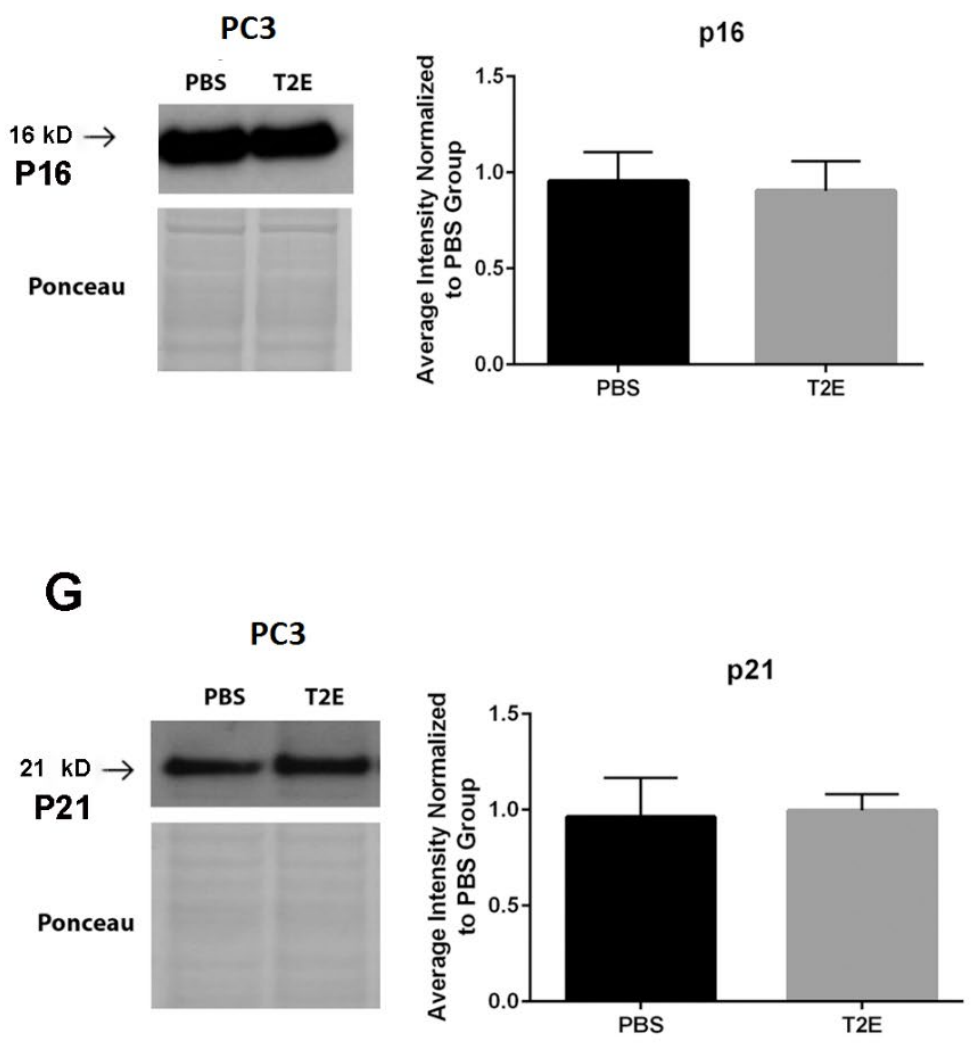
H
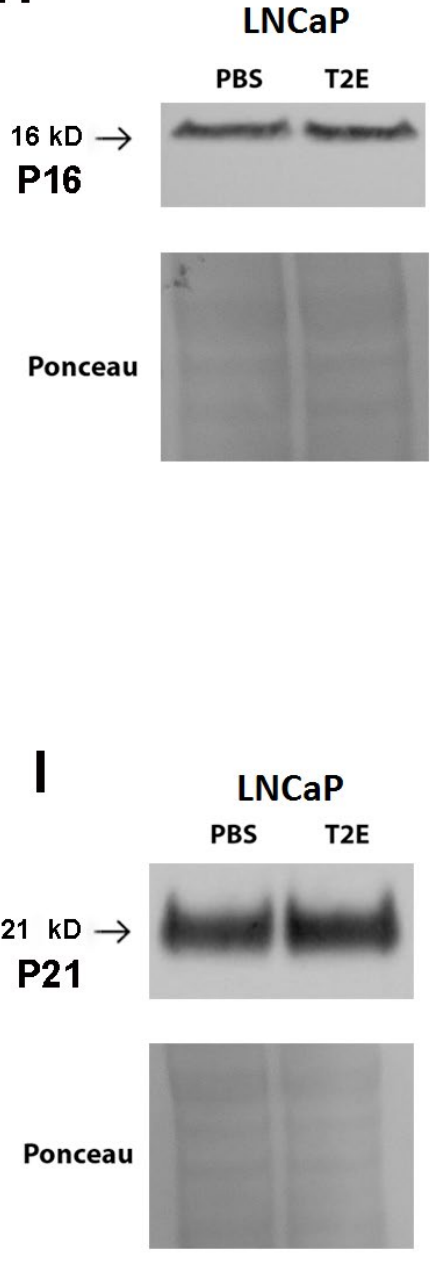

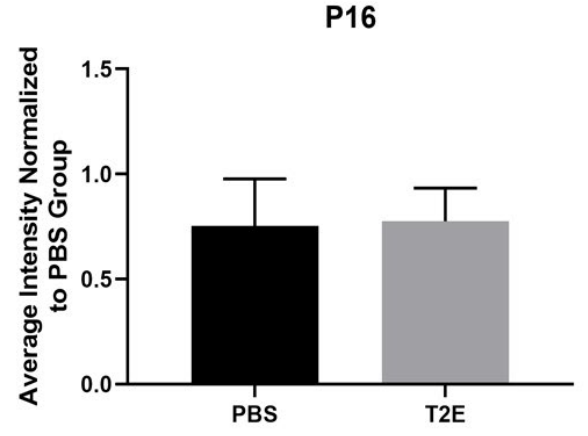

P21

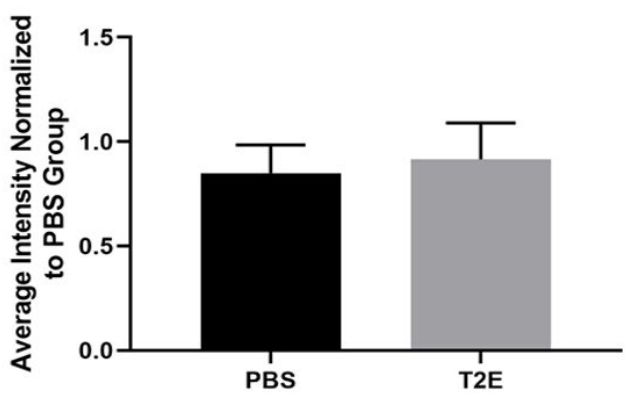




\subsection{MnTE-2-PyP enhances Ki67-negative cells but does not affect overall cell cycle distribution in vivo or in vitro in PC3 cells}

We wanted to determine how MnTE-2-PyP enhanced radiation-induced cell growth inhibition. Given the alterations of pRB phosphorylation states in MnTE-2-PyPtreated PC3 and LNCaP cells, the proliferation marker, Ki67, and DNA dye, DAPI, were used to determine whether prostate cancer cells were arrested in a particular cell cycle phase.

In PC3 cells, we found that MnTE-2-PyP enhanced a Ki67-negative population in $\mathrm{G}_{1} / \mathrm{G}_{0}(\sim 100 \%$ increase) and S phase ( $200 \%$ increase) as compared to PBS treatment; however, these increases no longer existed when radiation was applied (Figure 18B, C). In addition, neither MnTE-2-PyP nor radiation exposure affected cell cycle distribution (Figure 18D). Loss of Ki67 normally indicates cell quiescence, and RNA levels are greatly reduced in quiescent cells [168]. Therefore, we measured the RNA levels in these different conditions to determine whether MnTE-2-PyP treatment led to cell quiescence. We found that MnTE-2-PyP treatment caused a decrease of low-RNA population, which means MnTE-2-PyP did not suppress PC3 cells growth through cell quiescence (Figure 18E).

To further confirm above findings, we evaluated the cell cycle progression in PC3 cells by serum starvation to synchronize the cells at $G_{1} / G_{0}$ phase. The cell cycle was monitored for multiple time points in the first 48 hours and we did not observe any significant difference in cell cycle phase distribution between PBS and MnTE-2-PyP treatment (Figure 19).

We also measured the in vivo PC3 tumor cells that were treated with PBS or MnTE-

$2-\mathrm{PyP} \pm 10 \mathrm{~Gy}$, and no difference in cell cycle distribution was observed between the two conditions (Figure 20). Based on these results, we believe that cell quiescence or cell 
cycle progression disturbance is not the major contributor to MnTE-2-PyP-induced cell growth inhibition in PC3 cells. 
Figure 18. MnTE-2-PyP enhanced Ki67-negative population in PC3 cells but did not affect cell cycle progression. (A) PC3 cells were seeded in media containing PBS or 30 $\mu \mathrm{M}$ MnTE-2-PyP. After 24 hours, some groups of cells were exposed to 2 Gy radiation. After another 72 hours, cell numbers were enumerated. (B) Representative images of DAPI and Ki67 staining after 96 hours of MnTE-2-PyP treatment for PC3 cells. DAPI-only staining was used to gate the Ki67-negative population. (C) Ki67-negative population measurements in PC3 cells treated with MnTE-2-PyP or radiation or the combination of MnTE-2-PyP and radiation. (D) Cell cycle analyses for PC3 cells treated with MnTE-2PyP or radiation or the combination of the two. (E) Low-RNA population identified by pyronin and Hoechst staining. All data represent mean \pm SD from at least three independent experiments. ${ }^{*} p<0.05$ compared to PBS treatment; $\# p<0.05$ compared to PBS + 2Gy treatment. 


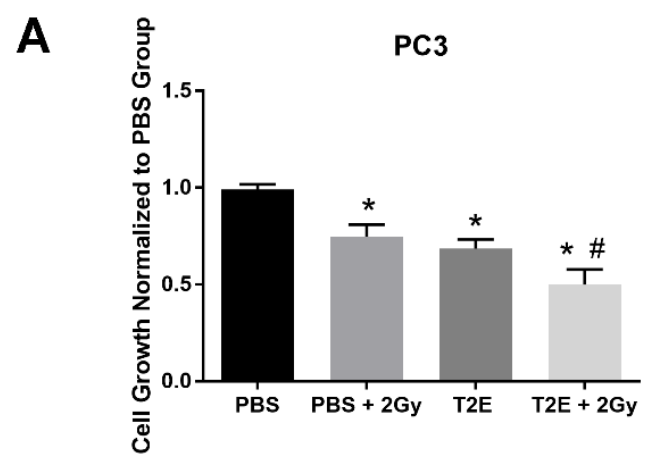

B
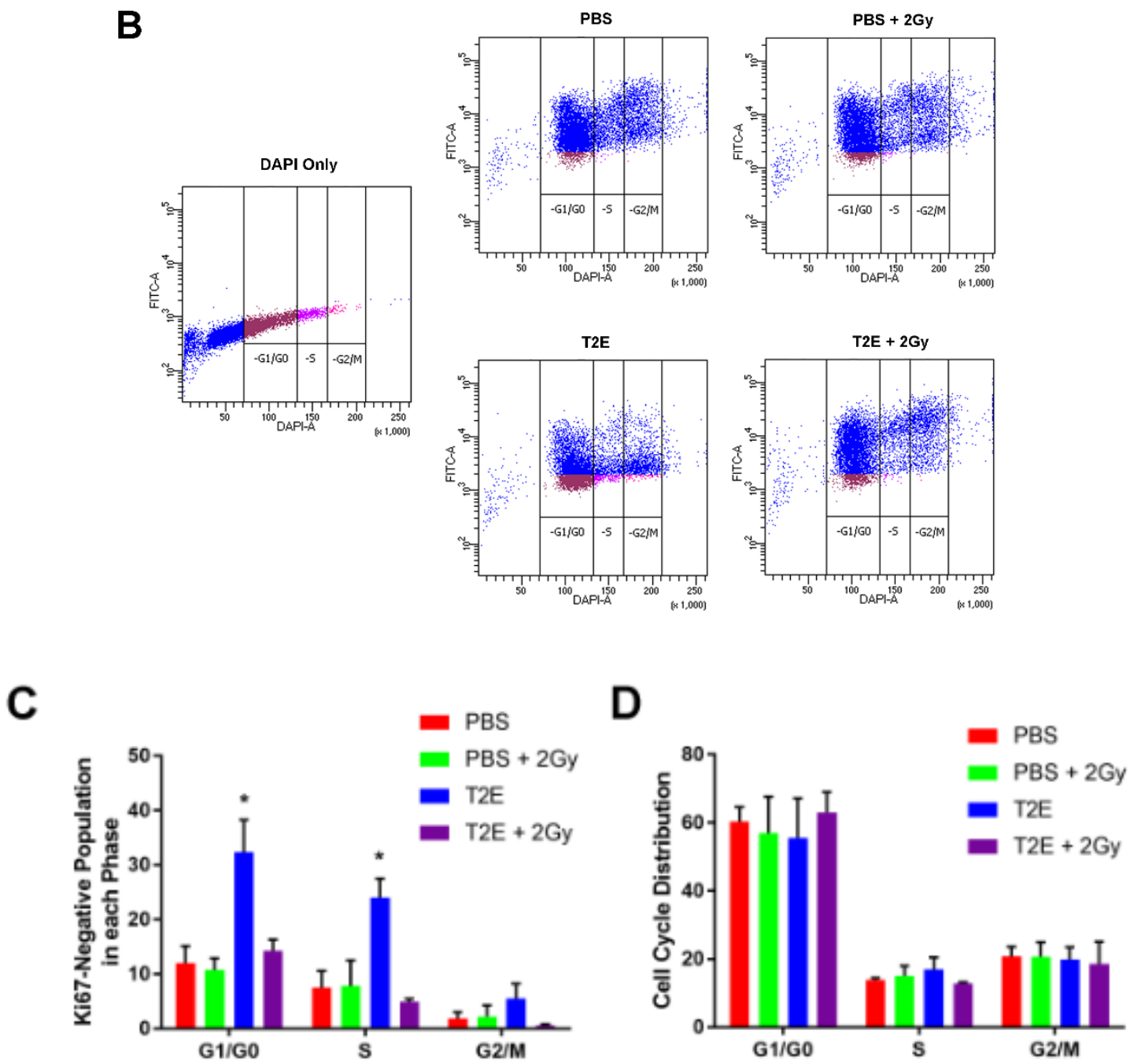


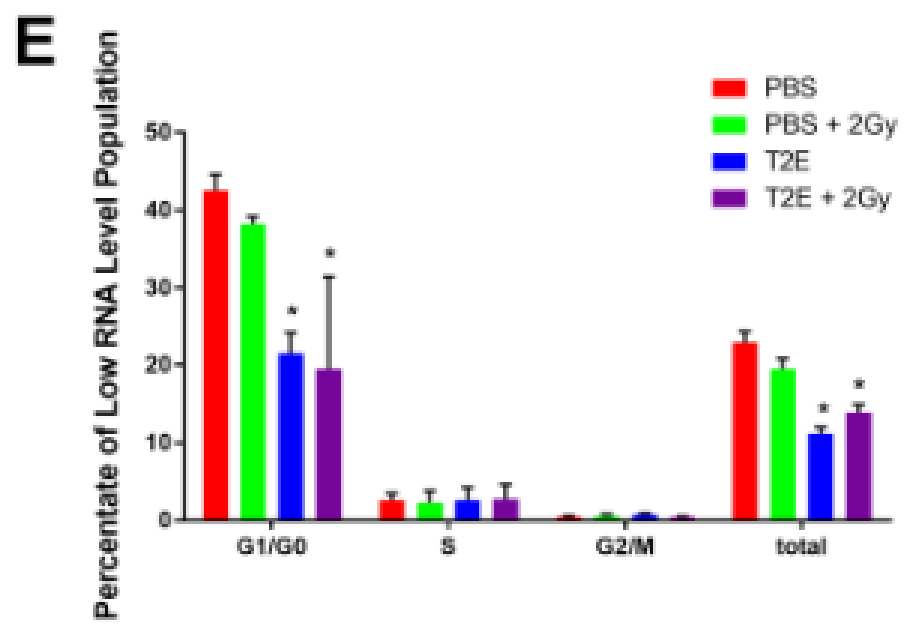


Figure 19. Effects of MnTE-2-PyP on PC3 cell cycle progression. PC3 cells were serum-starved for 48 hours and treated with PBS or MnTE-2-PyP (30 $\mu \mathrm{M})$. Cells were harvested at $0,12,16,20,24$, and 48 hours and stained for DAPI and Ki67. Left column: cell cycle distribution for PC3 cells; right column: Ki67-negative population for PC3 cells. 
Cell Cycle Distribution
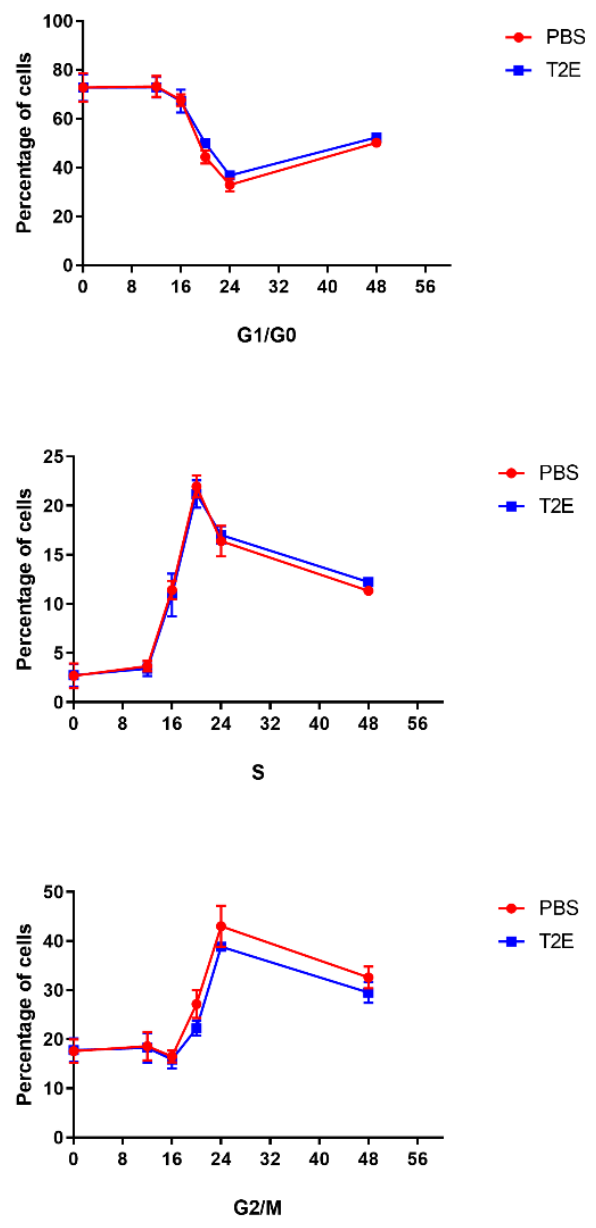

$\rightarrow$ PBS

- T2E

$\rightarrow$ PBS

- T2E
Ki67-negative population
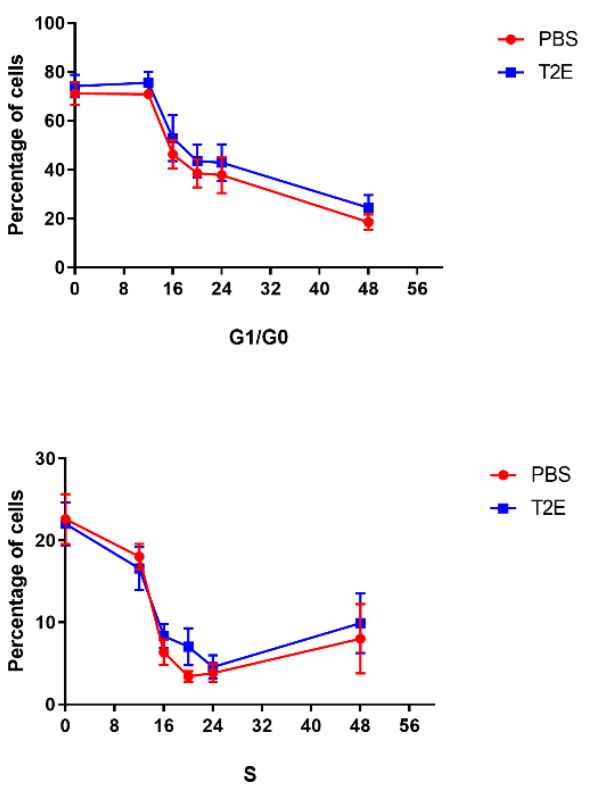

$\rightarrow$ PBS

- T2E

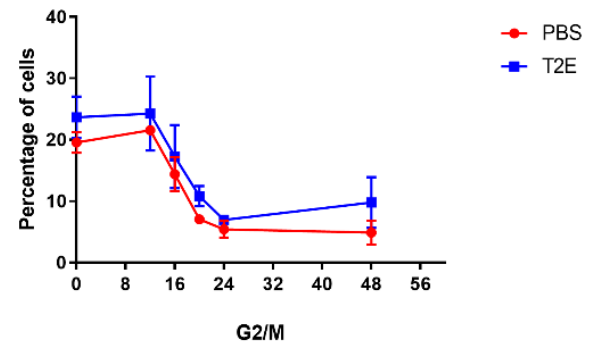


Figure 20. MnTE-2-PyP did not affect Ki67-negative population and cell cycle progression in PC3 tumor cells in vivo. PC3 tumors were isolated from tumor-bearing animals treated with PBS or MnTE-2-PyP (5 mg/kg, three times per week) + radiation (2 Gy per day, for five sequential days) treatment. A single-cell suspension was prepared as described in Materials and methods 2.5. In brief, tumor sections were minced and digested with Collagenase I and DNAse. Tumor pieces were strained through a $70 \mu \mathrm{m}$ strainer followed by $35 \mu \mathrm{m}$ strainer. Cell numbers were determined using a Coulter and cell viability was determined by Trypan Blue assay. Then Ki67 staining was performed and Ki67negative population was determined with FACS analysis. 

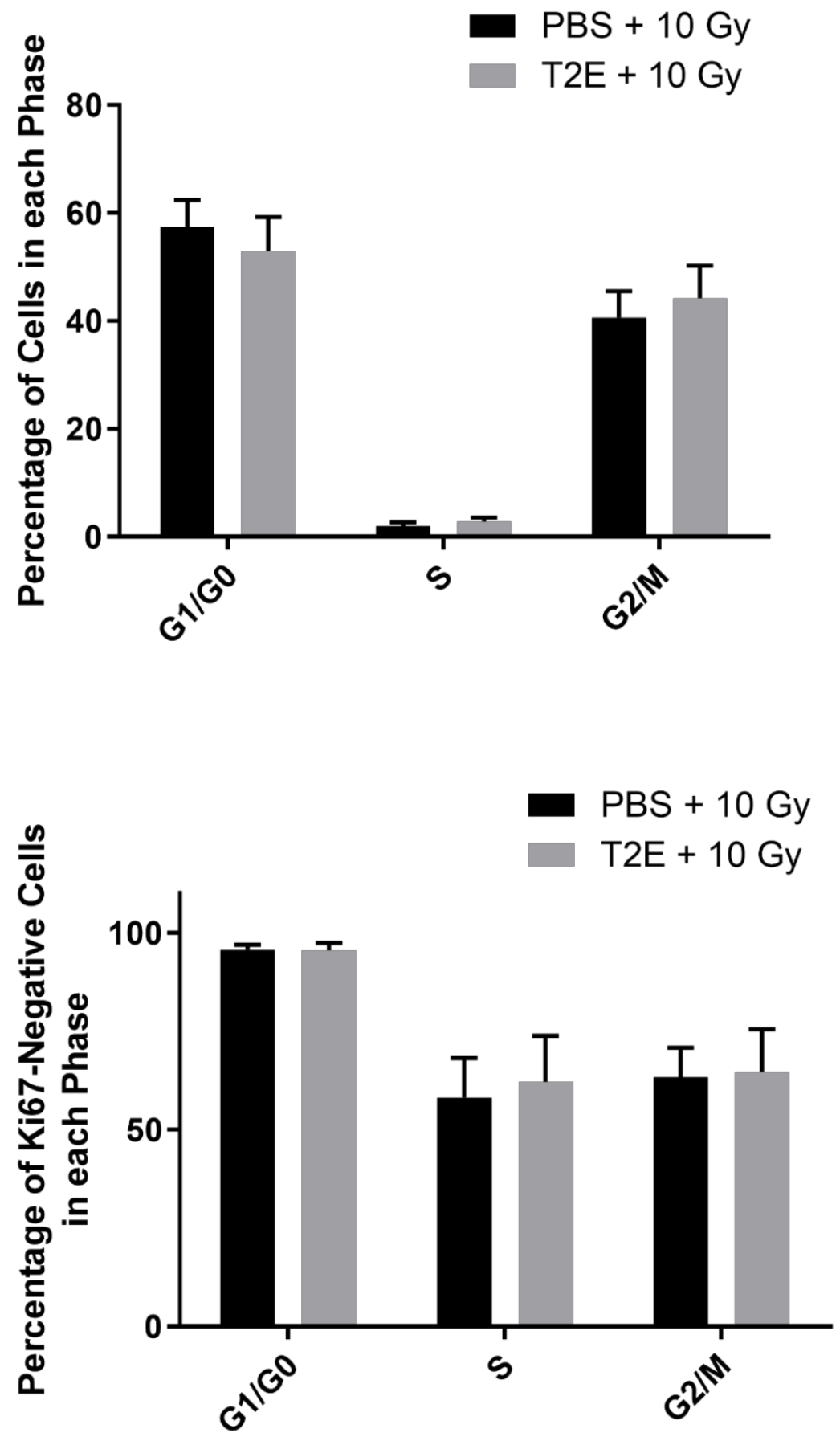


\subsection{MnTE-2-PyP enhances Ki67-negative cells and induces cell quiescence or cell death in LNCaP cells}

Correspondingly, the Ki67 levels were measured in LNCaP cells. As compared to PBS control, MnTE-2-PyP or radiation increased a Ki67-negative population in LNCaP cells at $G_{1} / G_{0}$ and $G_{2} / M$ phase, which achieved a maximum effect when the two treatments were combined (Figure 21B, C); however, the cell cycle distribution was still not altered (Figure 21D). In addition, we observed a significant increase of a sub- $\mathrm{G}_{1}$ population and a low RNA population in LNCaP cells with MnTE-2-PyP or radiation treatment (Figure 21E, F), and more than $95 \%$ of the low RNA population were included in the sub- $\mathrm{G}_{1}$ population. Therefore, the low RNA population in the sub- $\mathrm{G}_{1}$ population were either quiescent or dying. The above data indicate that the growth inhibition effects by MnTE-2-PyP in combination with radiation treatment are due to different mechanisms in PC3 and LNCaP cells. 
Figure 21. Effects of MnTE-2-PyP on cell cycle progression in combination with radiation in LNCaP cells. (A) LNCaP cells were seeded in media containing PBS or 30 $\mu \mathrm{M}$ MnTE-2-PyP. After 24 hours, some groups of cells were exposed to 2 Gy radiation. After another 72 hours, cell numbers were determined. (B) Representative images of DAPI and Ki67 staining after 96 hours of MnTE-2-PyP treatment for LNCaP cells. DAPI-only staining was used to gate the Ki67-negative population. (C) Ki67-negative population measurements in LNCaP cells treated with MnTE-2-PyP or radiation or the combination of the two. (D) Cell cycle analyses for LNCaP cells treated with MnTE-2-PyP or radiation or the combination of the two. (E) Measurement of the sub- $\mathrm{G}_{1}$ population in $\mathrm{LNCaP}$ cells (F) Total Low-RNA population identified in LNCaP cells by pyronin and Hoechst staining. All data represent mean \pm SD from at least three independent experiments. ${ }^{*} p<0.05$ compared to PBS treatment; \#p<0.05 compared to PBS + 2Gy treatment. 
A

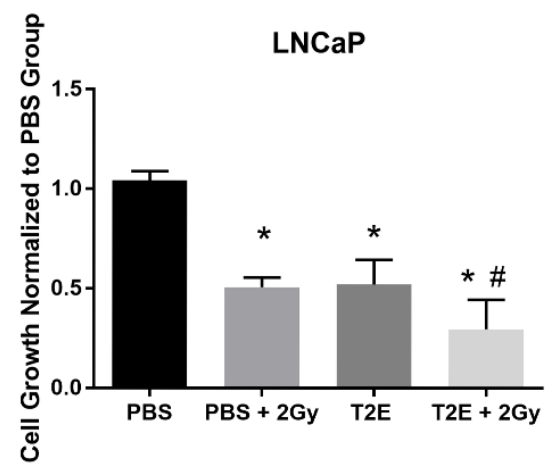

B
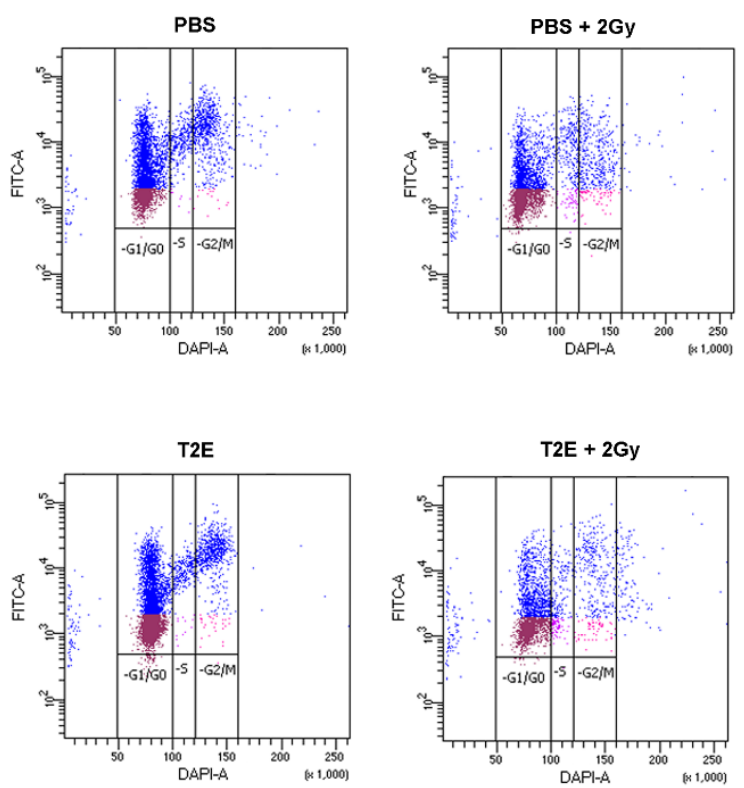

C

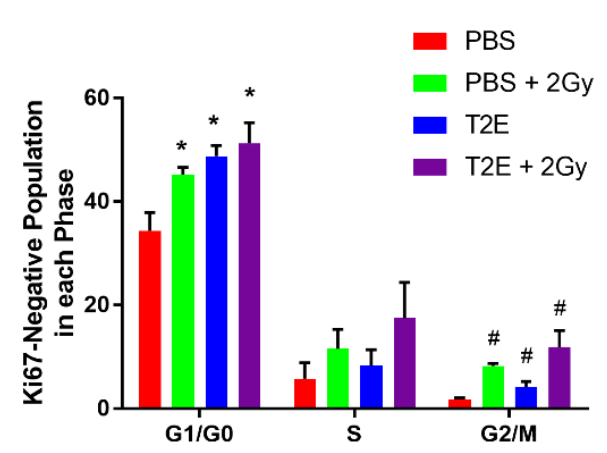

D

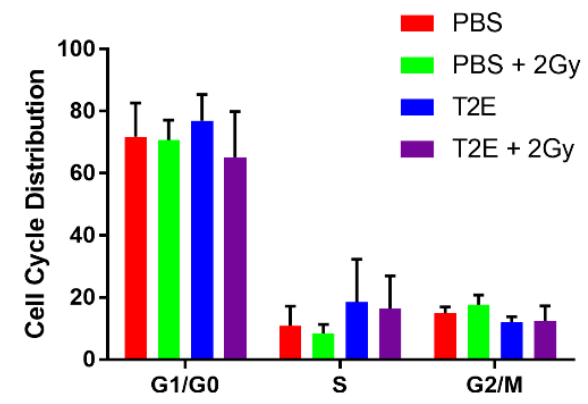


E

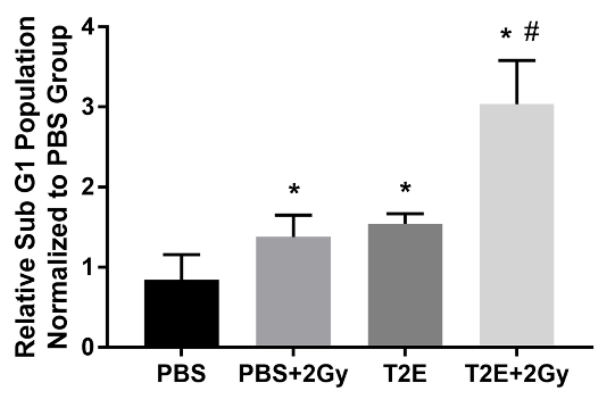

F

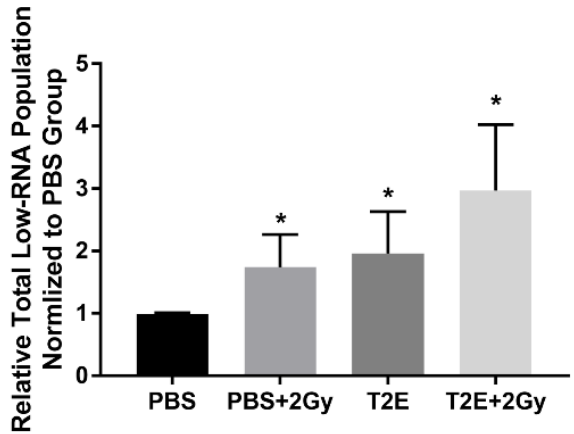




\subsection{MnTE-2-PyP treatment induces nuclear abnormalities in prostate cancer cells in vitro}

One of the leading causes of growth inhibition by radiation is DNA damage. Therefore, we next investigated whether MnTE-2-PyP caused more radiation-induced DNA damage in vitro (Figure 22A). We found that MnTE-2-PyP or radiation or the combination of the two significantly enhanced the percentage of PC3 cells with fragmented nuclei as compared to control group (Figure 22B left panel); however, in LNCaP cells, MnTE-2-PyP alone led to bi-nucleation, and radiation alone induced nuclear fragmentation. The combination of both treatments did not further show significant increase in either type of nuclear abnormality (Figure 22B right panel).

In order to determine whether nuclear fragmentation in PC3 cells and bi-nucleation in $\mathrm{LNCaP}$ cells resulted from enhanced $\mathrm{H}_{2} \mathrm{O}_{2}$ levels, both cell lines were transduced with catalase adenovirus. We found that in PC3 cells, the nuclear fragmentation caused by MnTE-2-PyP treatment could be rescued by catalase adenovirus transduction (Figure 22C, left panel). But in LNCaP cells, catalase transduction itself caused a similar level of bi-nucleation and did not rescue the effects caused by MnTE-2-PyP (Figure 22C, right panel). Therefore, we cannot determine whether $\mathrm{H}_{2} \mathrm{O}_{2}$ produced by MnTE-2-PyP directly caused bi-nucleation in LNCaP cells. However, these data indicate that $\mathrm{H}_{2} \mathrm{O}_{2}$ balance is involved in PC3 and LNCaP cell nuclear abnormalities.

To investigate whether MnTE-2-PyP potentially induces tumor cell death through induction of DNA damage, we stained PC3 cells treated with PBS/MnTE-2-PyP and radiation by using Calcein-AM. Calcein-AM is a non-fluorescent probe that permeates into cells where it is cleaved by intracellular esterases to produce stable fluorescent signals in living cells. Since 2 Gy radiation did not yield a sufficient number of cells with nuclear abnormalities and cell death for statistical analysis, we increased the radiation dose to 10 
Gy, which greatly enhanced nuclear fragmentation (60 70\%) and the cell death population with or without MnTE-2-PyP treatment (Figure 22D left panel). The Calcein-AM staining was reduced in MnTE-2-PyP-treated samples \pm radiation as compared to PBS-treated samples, specifically, for PC3 cells with nuclear fragmentation. MnTE-2-PyP significantly enhanced the low-Calcein-AM population as compared to control. This indicates that MnTE-2-PyP in combination with radiation can increase nuclear fragmentation, which leads to cell death or severe membrane damage (Figure 22D right panel). 
Figure 22. Nuclear abnormalities of prostate cancer cells treated with MnTE-2-PyP and radiation. (A) An example of bi-nucleated (green arrow), multi-nucleated (yellow arrow), and fragmented nucleus (red arrow) in PC3 cells. Cells were seeded in PBS or $30 \mu \mathrm{M}$ MnTE-2-PyP. After 24 hours, some groups was exposed to 2 Gy or 10 Gy radiation, and after 72 hours, cells were fixed onto slides by cytospin. (B) Quantification of nuclear abnormalities in PC3 and LNCaP cells in each condition. (C) Quantification of nuclear abnormalities in catalase-transduced PC3 and LNCaP cells with MnTE-2-PyP combined with radiation treatment. (D) Calcein-AM staining for PC3 cells with MnTE-2-PyP and radiation treatment. Percentage of dead cells in each condition after 96 hours treatment of PBS or $30 \mu \mathrm{M}$ MnTE-2-PyP \pm 10 Gy radiation. (E) Calcein-AM signal distribution in irradiated PC3 cells with nuclear fragmentation. All data represent mean \pm SD from at least three independent experiments. ${ }^{*} p<0.05$ compared to PBS treatment; $\# p<0.05$ compared to PBS + 10 Gy treatment. 
A

Phase Contrast

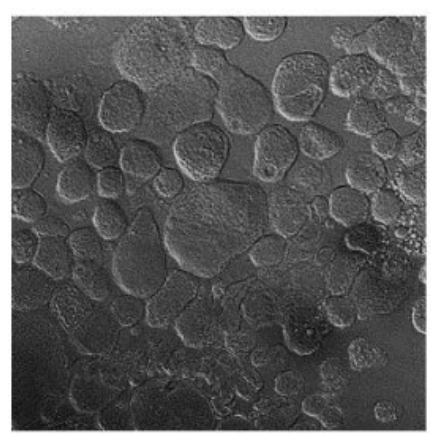

DAPI

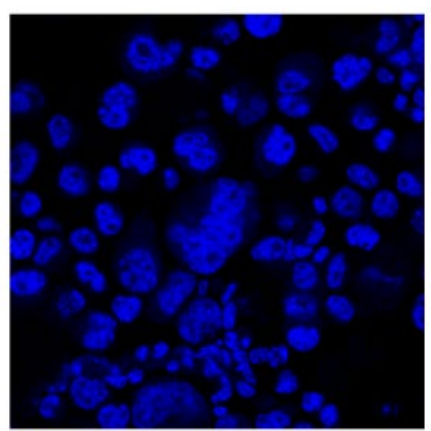

Merge

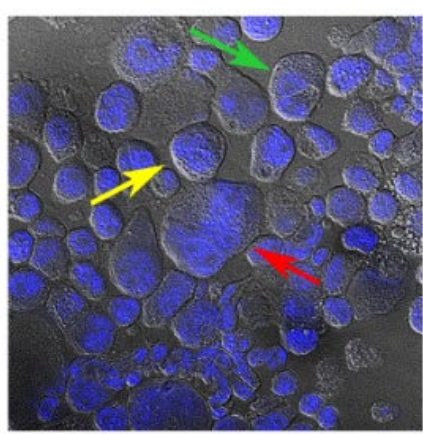

B
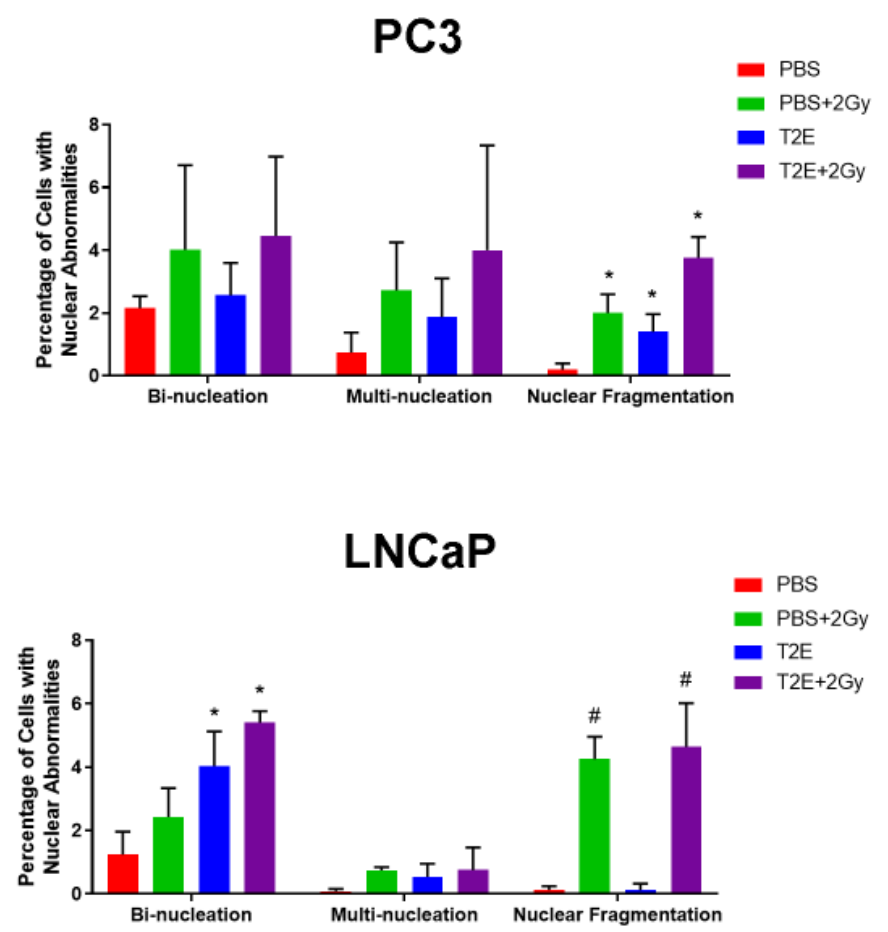
C
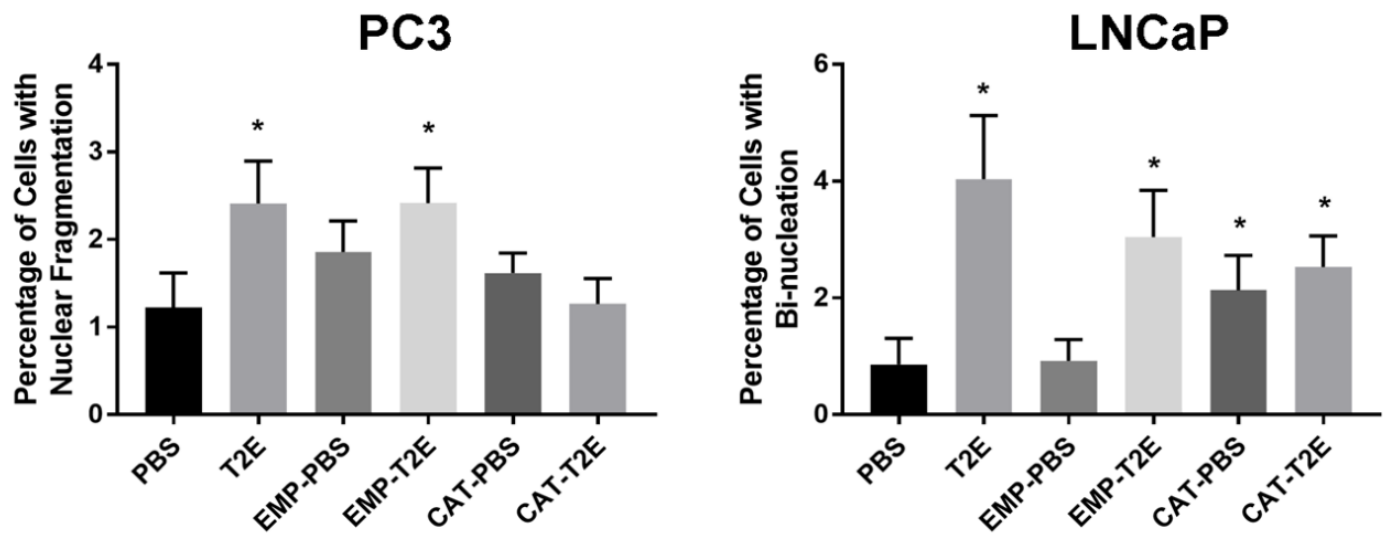

D
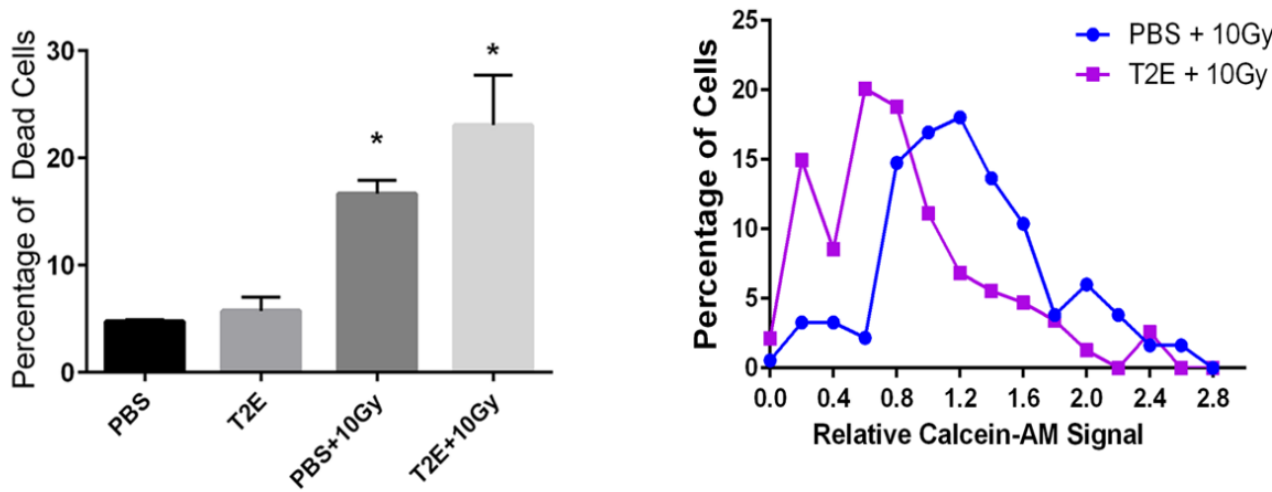


\subsection{MnTE-2-PyP treatment induces nuclear abnormalities in prostate cancer cells in vivo}

To investigate the effects of MnTE-2-PyP with radiotherapy in vivo, the PC3 tumor cells were injected orthotopically into the prostates of athymic nude mice. MnTE-2-PyP combined with radiation ( $2 \mathrm{~Gy} \times 5$ days) significantly decreased the tumor size and weight as compared to PBS and radiation treatment (Figure 23A). We measured the phosphorylation levels of cyclin D1 and pRB in PC3 tumor, but there was no significant difference between radiation alone and MnTE-2-PyP combined with radiation groups (Figure 23B). We also investigated the DNA damage and nuclear abnormalities in the PC3 tumor. There was a significant increase in $\mathrm{yH} 2 \mathrm{AX}$ staining in MnTE-2-PyP and radiation treatment as compared to radiation alone (Figure 23C, D), indicating there is more DNA damage in PC3 tumor cells treated with MnTE-2-PyP. Correspondingly, both multinucleation and nuclear fragmentation increased, with a decrease in bi-nucleation, in the MnTE-2-PyP and radiation group as compared to the radiation alone group. These above results indicate that in an in vivo PC3 tumor model, MnTE-2-PyP with radiation treatment causes more DNA damage and nuclear abnormalities, which could be partially responsible for tumor growth arrest, rather than pRB activation. 
Figure 23. Effects of MnTE-2-PyP with radiation in PC3 tumors in vivo. (A) Left panel: In vivo PC3 tumor size; right panel: In vivo PC3 tumor weight after PBS or MnTE-2-PyP + 5 days $\times 2$ Gy/day treatment. (B) Left panel: phosphorylation level of cyclin D1 in PC3 tumor samples; right panel: phosphorylation level of pRB in PC3 tumor samples. (C) Representative images of $\mathrm{yH} 2 \mathrm{AX}$ positive cells in each condition. Each arrow indicates a single cell with $\mathrm{yH} 2 \mathrm{AX}$ positive staining, part of the positive cells were shown. (D) Quantification of $\mathrm{yH} 2 \mathrm{AX}$-positive cells in PC3 tumor sections. Each quantified image had a similar cell density. (E) Representative images of nuclear abnormalities in PC3 tumor samples with PBS or MnTE-2-PyP + 10 Gy radiation treatment. (F) Quantification of $(E)$, around 1000 cells isolated from each animal's tumor were included in the analysis. For animal experiments, $\mathrm{n}=10$ for both PBS +10 Gy and MnTE-2-PyP +10 Gy. ${ }^{*} p<0.05$ compared to PBS + 10 Gy treatment. 
A
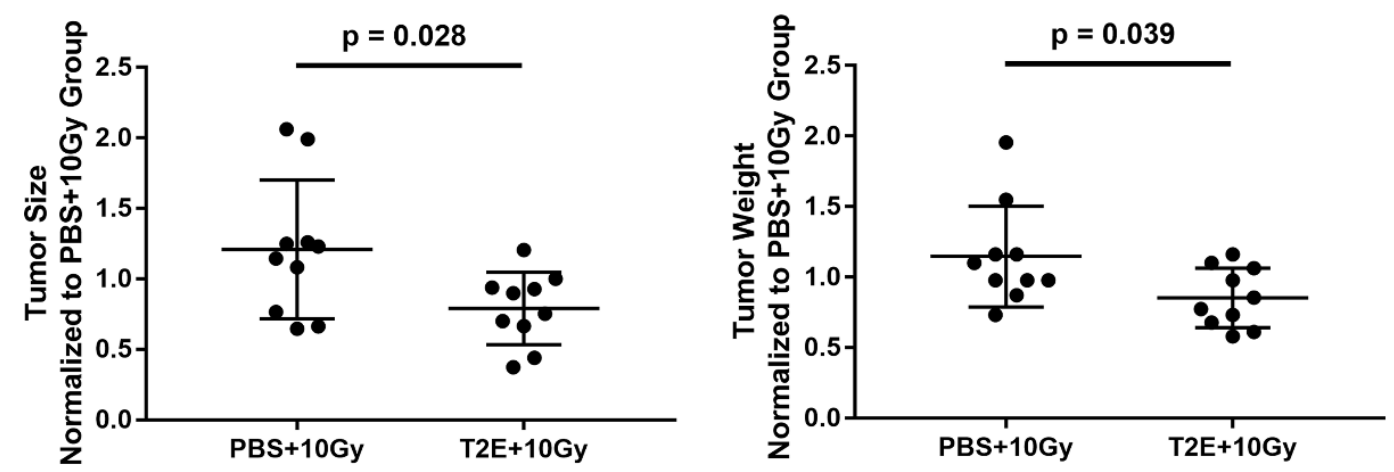

B
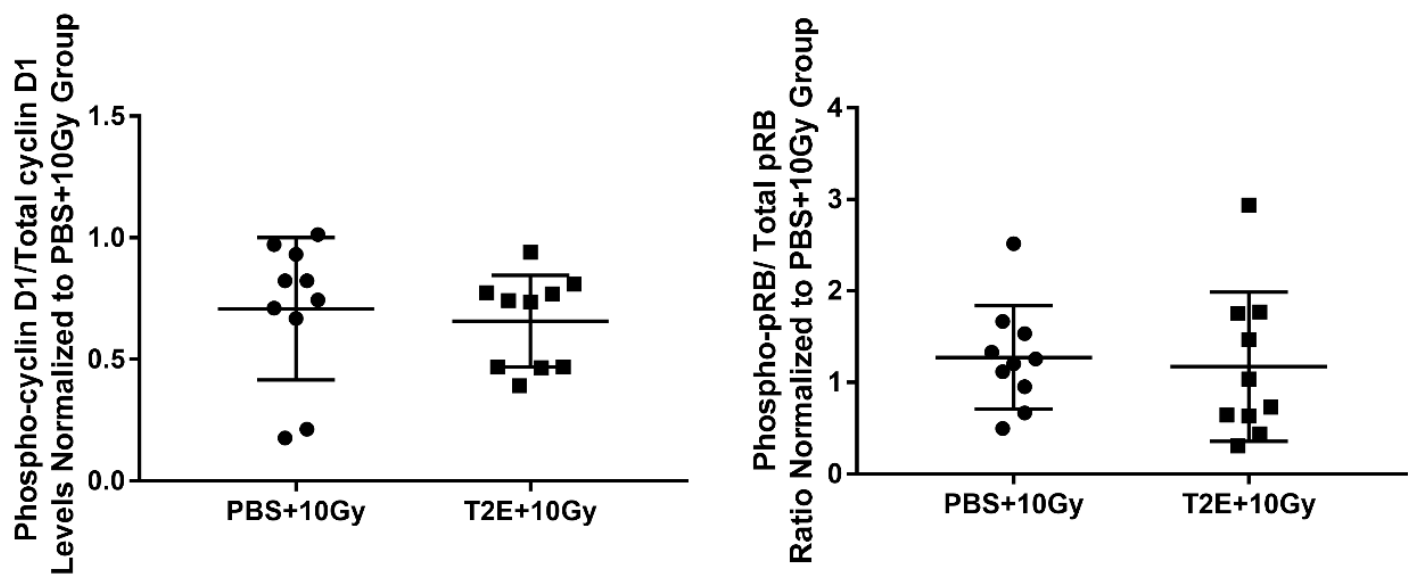

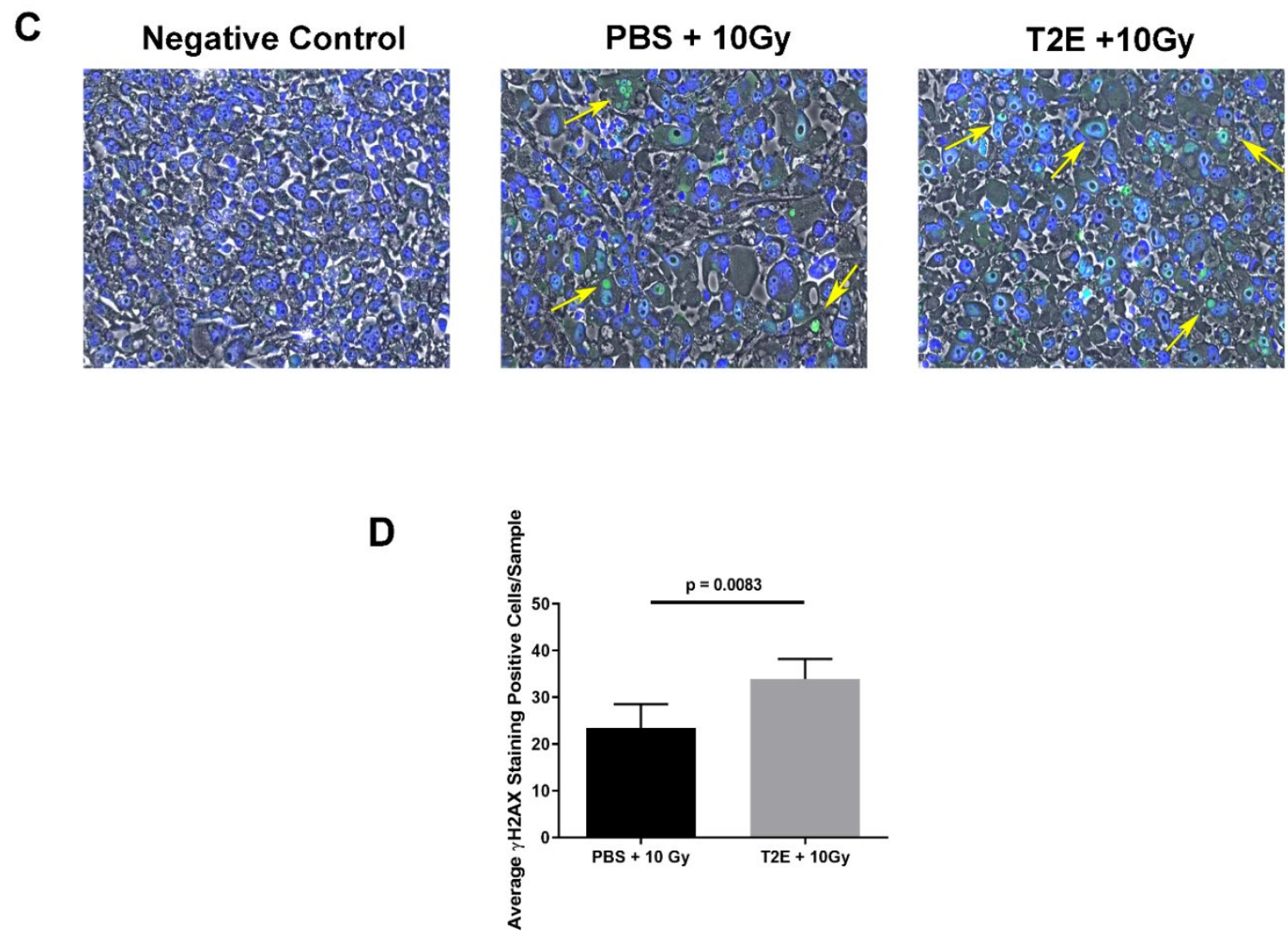
E

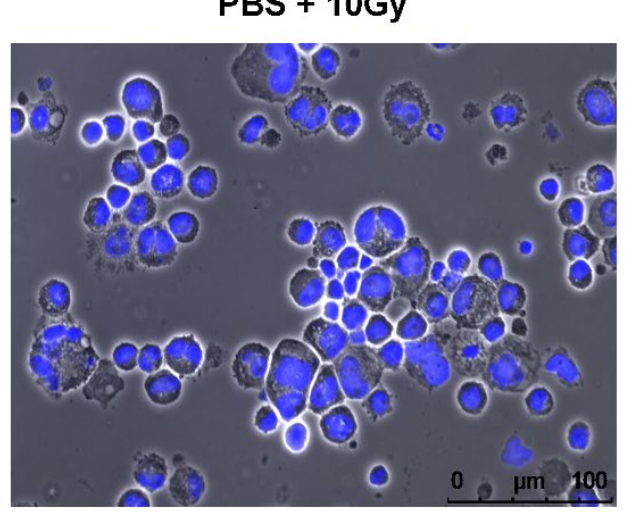

T2E +10Gy

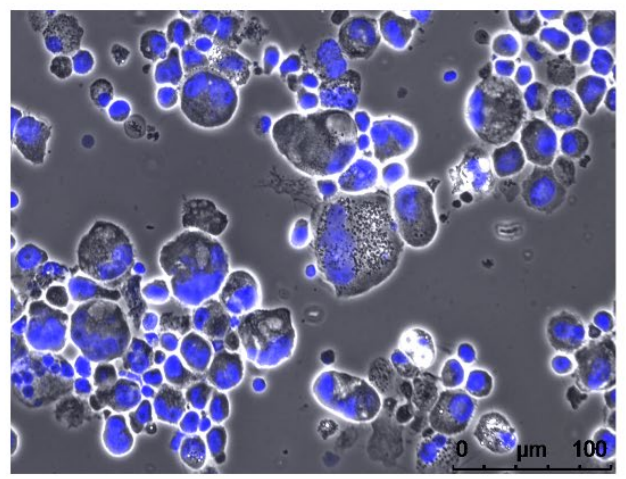

F

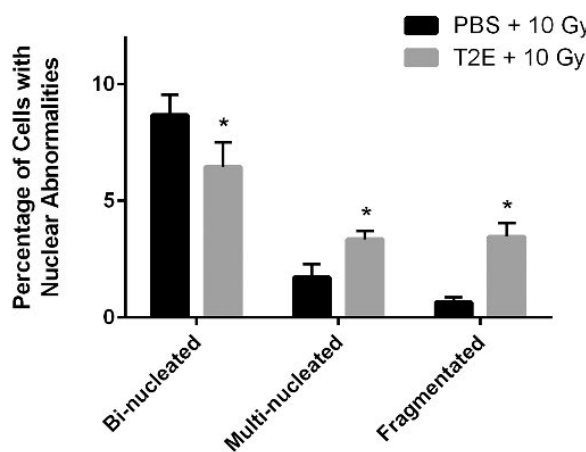




\subsection{MnTE-2-PyP does not induce the Ki67-negative population in TrampC cells}

TrampC is an aggressive mouse prostate cancer cell line. We have shown that TrampC cells growth was strongly inhibited by MnTE-2-PyP by day 3, which is around 24 hours earlier than PC3 and LNCaP cells. To determine whether MnTE-2-PyP also increase Ki67-negative population in TrampC cells, we performed Ki67/DAPI staining in TrampC cells. We found that neither MnTE-2-PyP nor radiation affected Ki67-negative population in TrampC cells (Figure 24). 
Figure 24. Effects of MnTE-2-PyP on cell cycle progression in combination with radiation in TrampC cells. TrampC cells were seeded in media containing PBS or $30 \mu \mathrm{M}$ MnTE-2-PyP. After 24 hours, some groups of cells were exposed to 2 Gy radiation. After another 72 hours, cell numbers were determined. Cells were stained with Ki67 and the Ki67-negative population measurements in TrampC cells treated with MnTE-2-PyP or radiation or the combination of the two were analyzed and shown. All data represent mean \pm SD from at least three independent experiments. 


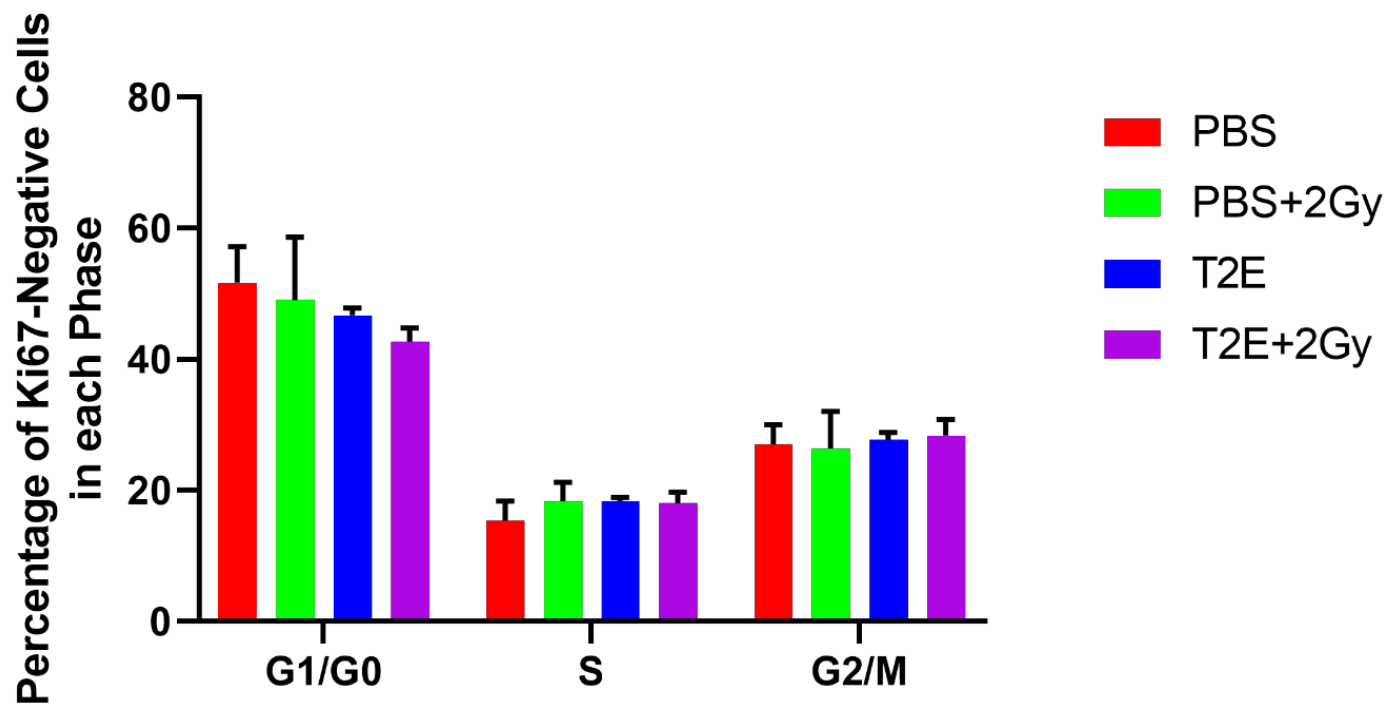




\subsection{Identifying MnTE-2-PyP-induced reversible thiol modifications in PC3 cells (lodoTMT-switch assay)}

We have shown MnTE-2-PyP led to protein oxidation in PC3 cells using the BIAM assay (Figure 13); however, the sensitivity and accuracy of the BIAM assay set a limit to mass spectrometry analysis and we only identified one target, PP1CB, among three replicates. To better identify protein targets that are affected by MnTE-2-PyP treatment in PC3 cells, we used the newly invented lodoTMT probe and performed an lodoTMT-switch assay (Figure 24A). The accuracy of results was further enhanced by two sets of independent experiments (Figure 24B). Consistently, we found that MnTE-2-PyP led to thiol oxidation in PC3 cells, and specifically, mitochondrial and protein synthesis-related proteins were significantly affected (Table 2). These data confirmed that MnTE-2-PyPinduced $\mathrm{H}_{2} \mathrm{O}_{2}$ levels increase are contributing to protein oxidation in PC3 cells; however, it is worth mentioning that not all modified thiols can result in protein functional or structural alterations, and the identified targets in lodoTMT-switch assay are not directly associated with MnTE-2-PyP-induced cell growth inhibition. In addition, the thiol modification may not be limited to $\mathrm{H}_{2} \mathrm{O}_{2}$ oxidation, glutathionylation can also occur [169]. Therefore, results in Table 2 indicate that mitochondrial function and protein synthesis can be two major areas that are affected by MnTE-2-PyP treatment, but each target still needs to be evaluated for activity through functional analysis. 
Figure 24. lodoTMT-switch assay in PC3 cells. (A) PC3 cells were treated with PBS or MnTE-2-PyP for 48 hours and protein lysates were processed for lodoTMT probe enrichment. Two types of labeling were performed for two independent experiments: 126 131 lodoTMT labeling and 126 lodoTMT/Non-labeled TMT labeling. The 126 131 lodoTMT labeling provided relatively more accurate results of the percentage of reversible thiol modifications between PBS and MnTE-2-PyP-treated samples; the 126 lodoTMT/Non-labeled TMT labeling provided less accurate but larger group of results. Enriched proteins were sent for mass spectrometry analysis. (B) Peptides that contained the same type of modifications from both experiments (9 replicates from experiment 1 and 6 replicates from experiment 2) were overlapped based on the criteria of $>75 \%$ consistency, and then statistical significance of these modifications were calculated by Perseus. 
A

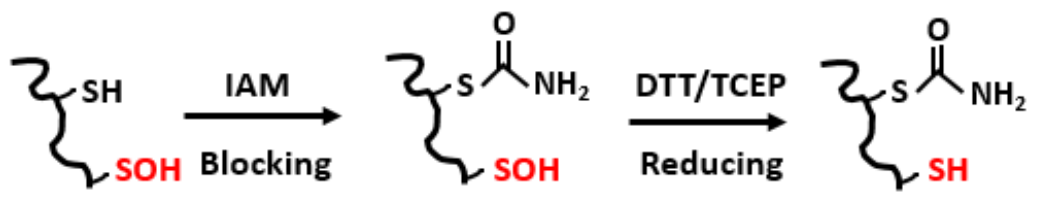
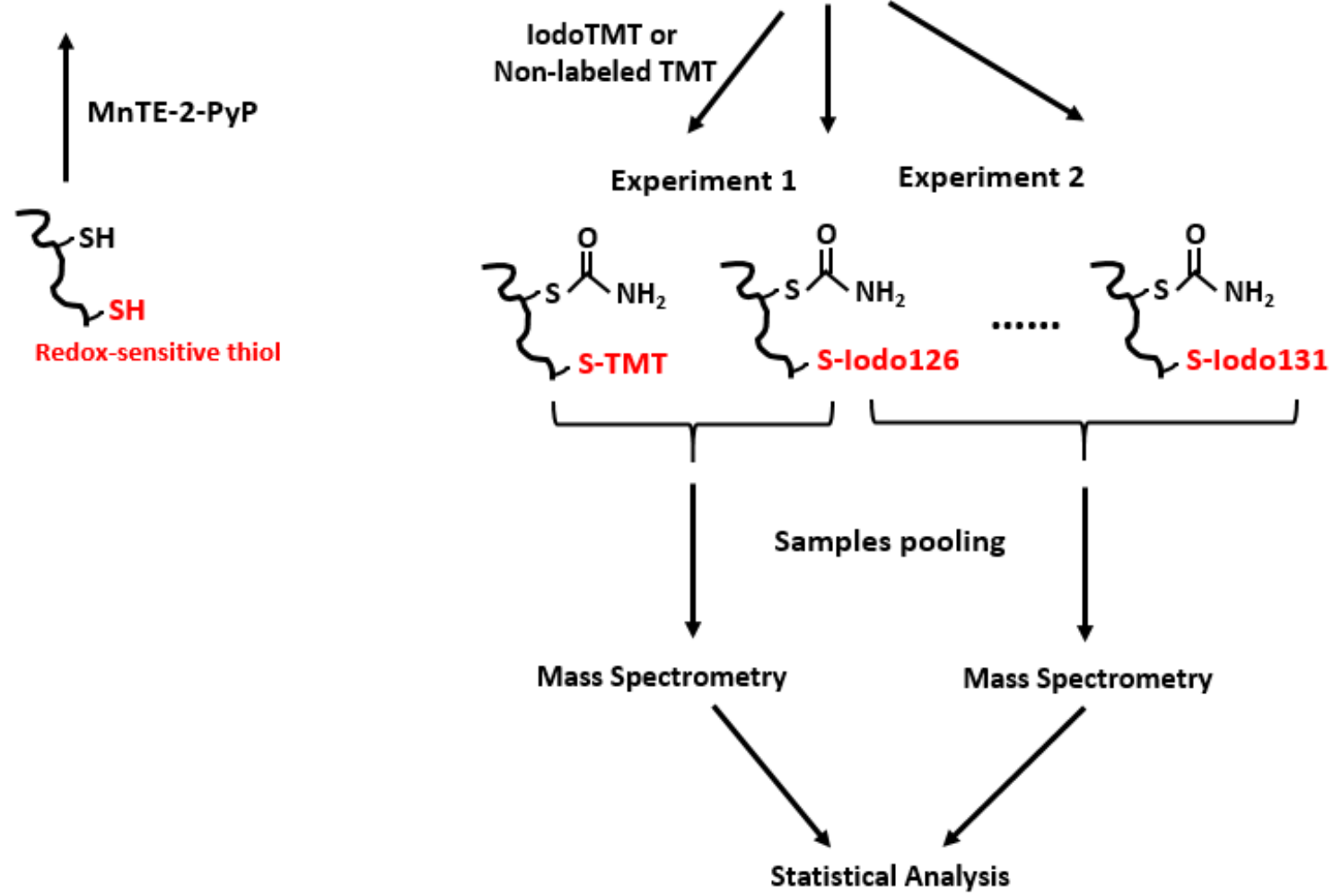
B

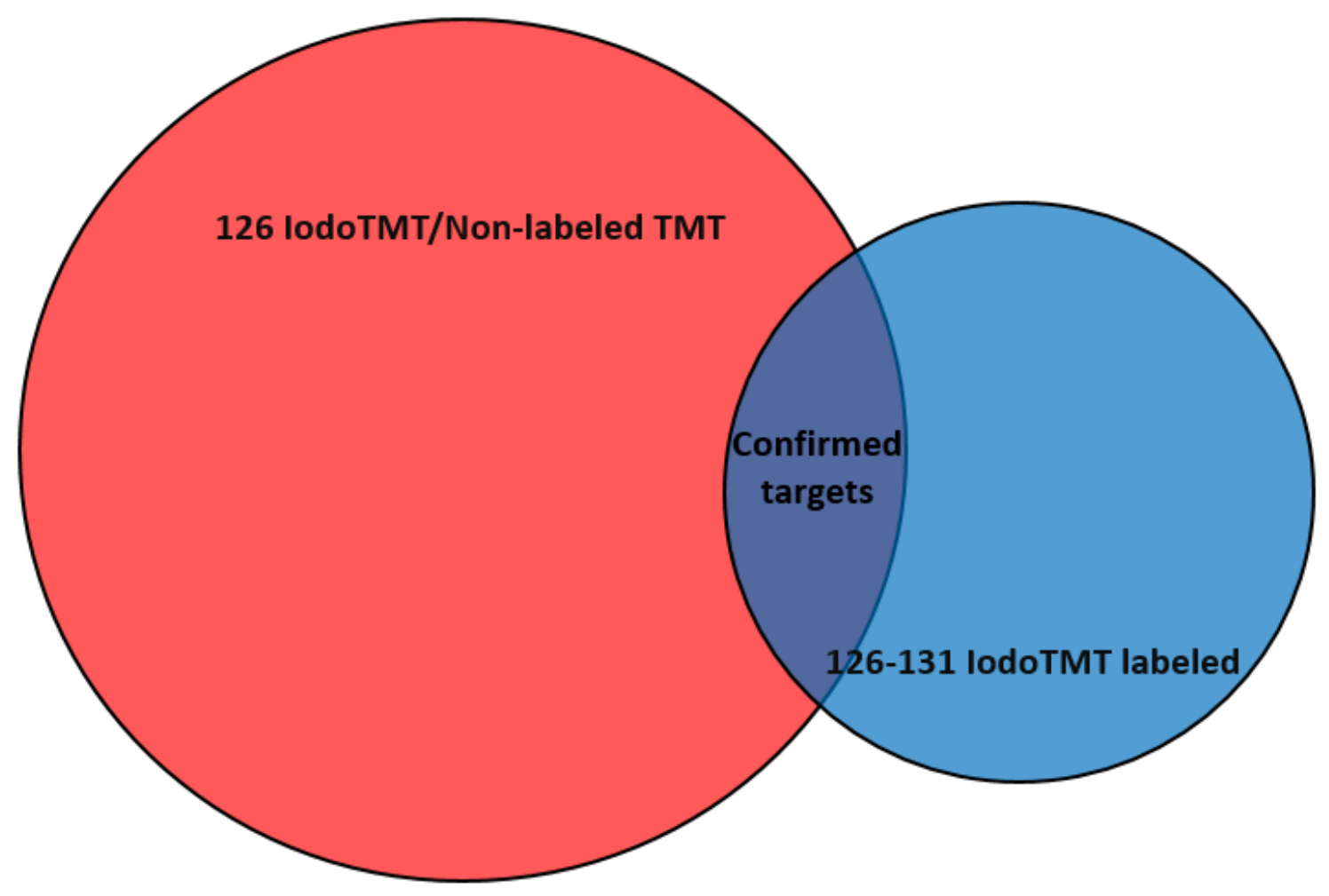


Table 2: Protein peptide targets with reversible redox modifications

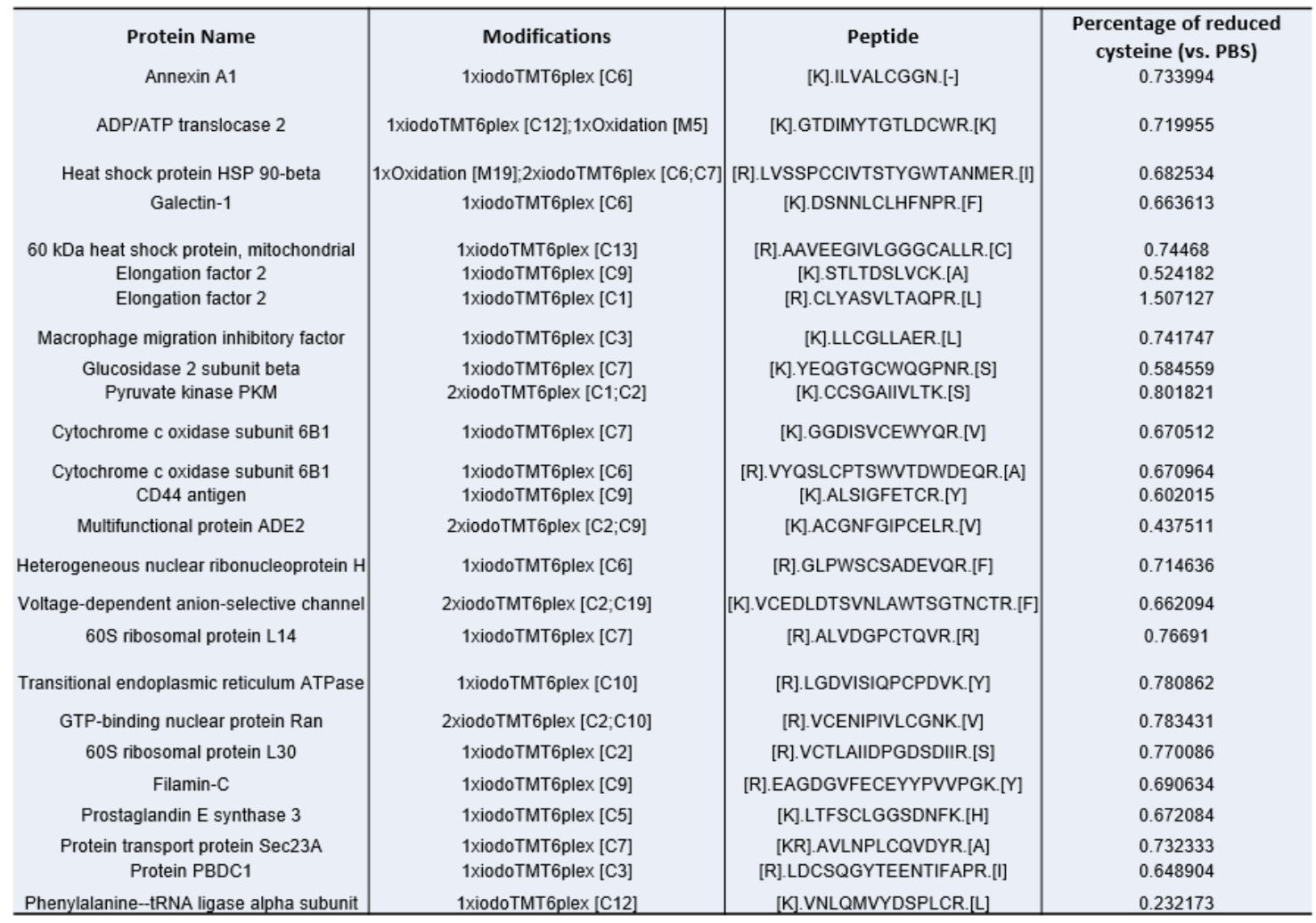




\subsection{MnTE-2-PyP does not affect PKM activity in PC3 cells}

Pyruvate kinase catalyzes the last step of glycolysis that converts phosphoenolpyruvate (PEP) to pyruvate and generates one molecule of ATP [170]. Based on the lodoTMT-switch assay results (Table 2), we found pyruvate kinase 2 (PKM) showed reversible thiol modifications under MnTE-2-PyP treatment. PKM is the only one of the two pyruvate kinase isoforms that is sensitive to oxidation [170]. Since glycolysis plays an important role in cancer cell metabolism, we first investigated whether total pyruvate kinase activity was affected in PC3 cells with MnTE-2-PyP or radiation treatment. We found that neither 96 hours MnTE-2-PyP treatment nor 2 Gy radiation affected total pyruvate kinase activity (Figure 25). To specifically determine the activity of PKM under MnTE-2-PyP treatment, we treated PC3 cells with increasing doses of $\mathrm{H}_{2} \mathrm{O}_{2}$ to locate the oxidized forms of PKM [171]; however, one hour treatment of up to $10 \mathrm{mM} \mathrm{H}_{2} \mathrm{O}_{2}$ did not lead to significant PKM oxidation (Figure 26). Although this could be due to an issue of limited sensitivity for non-reducing SDS-PAGE gel, in combination with the total pyruvate kinase activity assay results, we believe that the PKM modification detected by lodoTMTswitch assay did not directly contribute to PKM2 activity alteration. 
Figure 25. PKM activity was not altered by MnTE-2-PyP treatment in PC3 cells. PC3 cells were seeded in media containing PBS or $30 \mu \mathrm{M}$ MnTE-2-PyP. After 24 hours, some groups of cells were exposed to 2 Gy radiation. After another 72 hours, cellular PKM activity were determined. All data represent mean \pm SD from at least three independent experiments. 


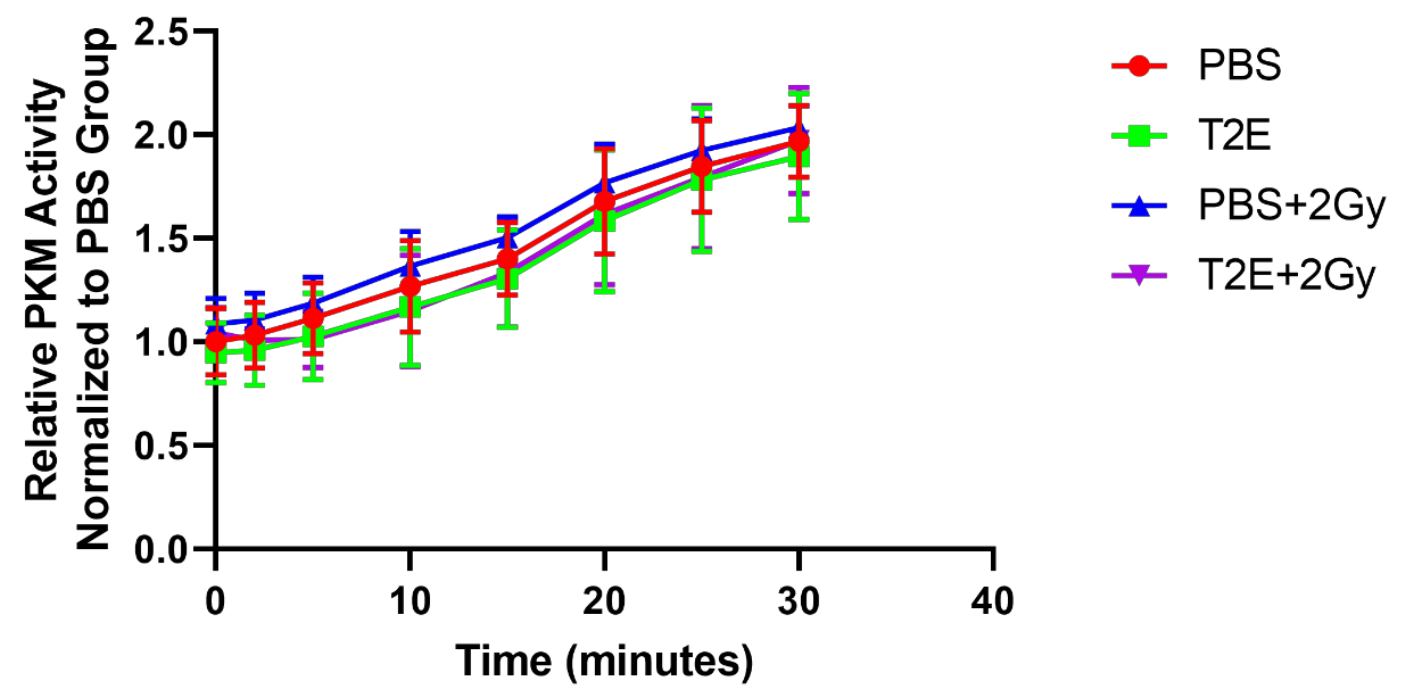

30 Minutes

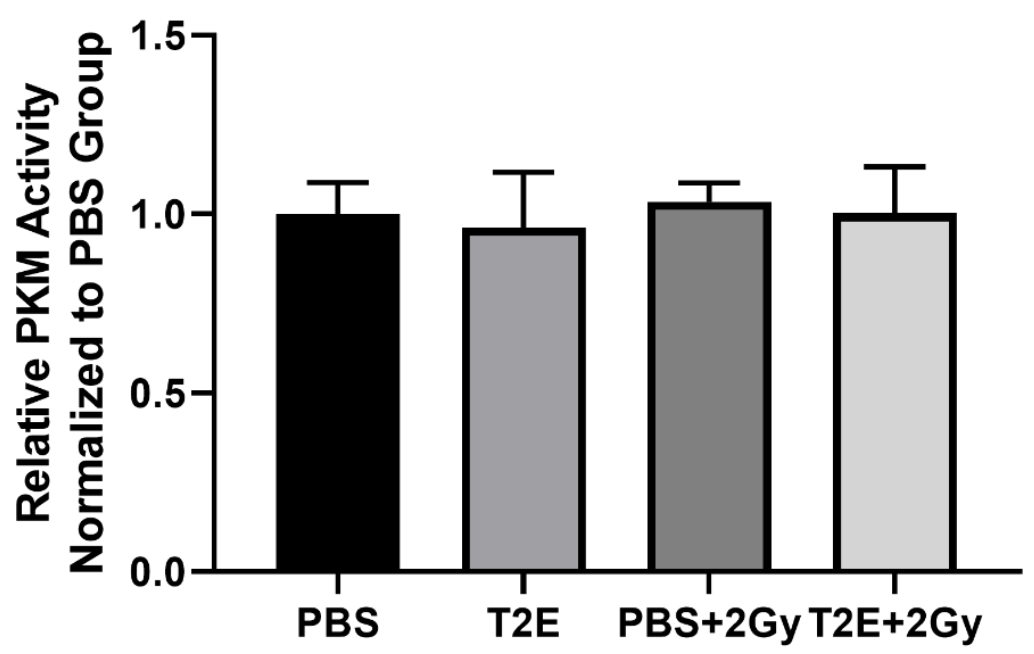


Figure 26. Identifying oxidized PKM2 isoforms in PC3 cells with $\mathrm{H}_{2} \mathrm{O}_{2}$ treatment. PC3 cells were treated with $0,0.1$, 1 or $10 \mathrm{mM} \mathrm{H}_{2} \mathrm{O}_{2}$ for 1 hour and a non-reducing SDS-PAGE gel was run to detect oxidized isoforms of PKM2. This figure represents two technical replicates. 


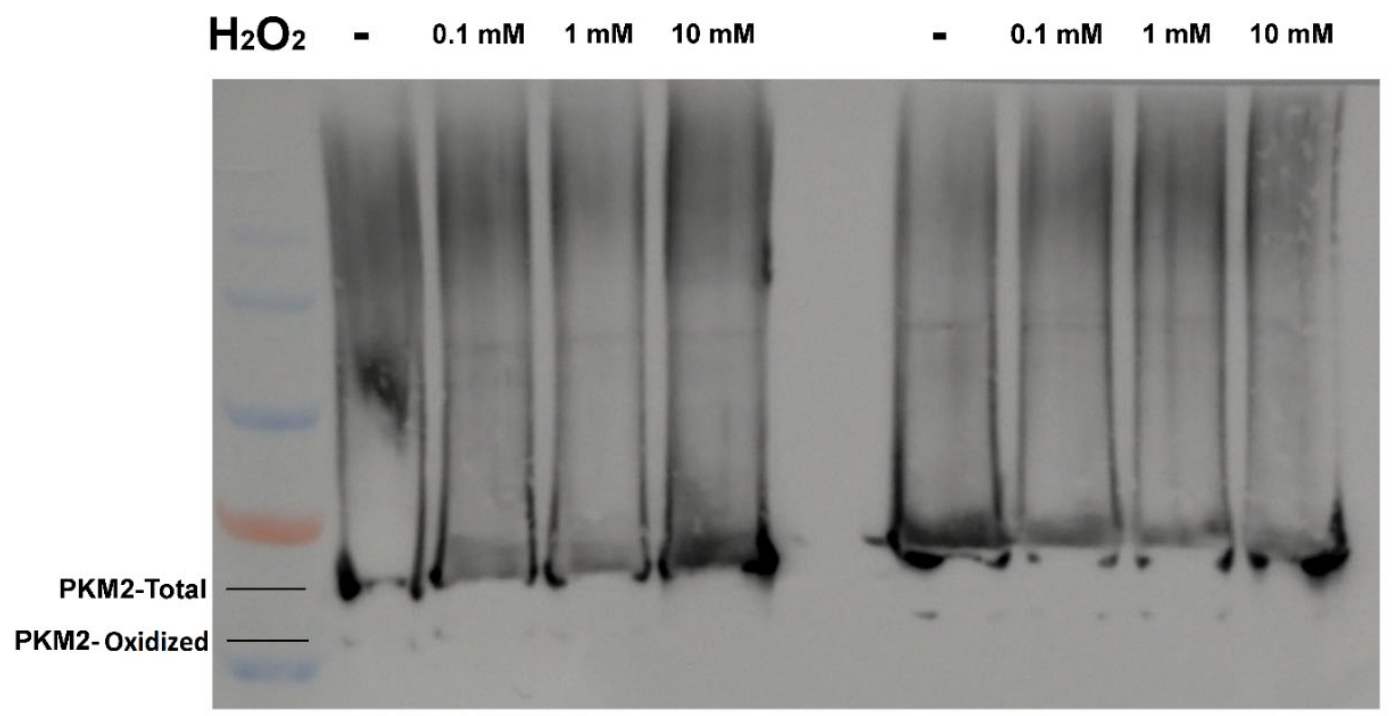




\subsection{The effects of MnTE-2-PyP on prostate cancer energy metabolism}

Based on the lodoTMT-switch assay results (Table 2), we found that many proteins involved in energy metabolism displayed reversible modifications, including ADP/ATP translocase 2, pyruvate kinase PKM, cytochrome c oxidase subunit 6B1, CD44 antigen, and the voltage-dependent anion-selective channel. Some of them are located in the mitochondria, which is the central organelle for oxidative phosphorylation. We measured the expression levels of 5 complexes that make up the electron transport chain (ETC) in PC3 cells with PBS or MnTE-2-PyP treatment. We found that complex 2 (succinate dehydrogenase) levels were significantly reduced by MnTE-2-PyP after 96 hours treatment (Figure 27). Although there was a trend of increasing levels of complex 1 (NADH ubiquinone oxireductase) and complex $3(\mathrm{CoQH} 2-$ cytochrome c reductase) by MnTE-2PyP treatment, the increase was not statistically significant.

To further determine whether MnTE-2-PyP altered energy metabolism in prostate cancer cells, we measured both oxidative phosphorylation (OXPHOS) and glycolysis (ECAR) in PC3 and LNCaP cells using the Seahorse instrument. We found that MnTE-2PyP and radiation triggered different responses in these two cells lines: in PC3 cells, either MnTE-2-PyP or radiation increased both OXPHOS and glycolysis, and the combination of the two treatments achieved maximal enhancement (Figure 28); however, in LNCaP cells, MnTE-2-PyP or radiation alone could still enhance OXPHOS, but the combination of the two treatments reduced the OXPHOS back to PBS level. In addition, MnTE-2-PyP alone is able to mildly suppress glycolysis in $\mathrm{LNCaP}$ cells as compared to other treatments (Figure 29). These results indicate that the OXPHOS alterations could be a response due to MnTE-2-PyP-induced stimulus, and glycolysis changes may not play as an important role in growth inhibition effects of MnTE-2-PyP. 
Figure 27. MnTE-2-PyP treatment reduced complex 2 expression levels in PC3 cells. PC3 cells were treated with PBS or MnTE-2-PyP for 48 or 96 hours, and all 5 complexes of ETC were detected by western blots. (A) A representative western blot of 5 ETC complexes (left panel) and Ponceau staining (right panel) in PC3 cells with PBS or MnTE2-PyP treatment. (B) Densitometry of expression levels of each of the 5 complexes. All data represent mean $\pm S D$ from at least three independent experiments. ${ }^{*} p<0.05$ compared to PBS treatment. 
A
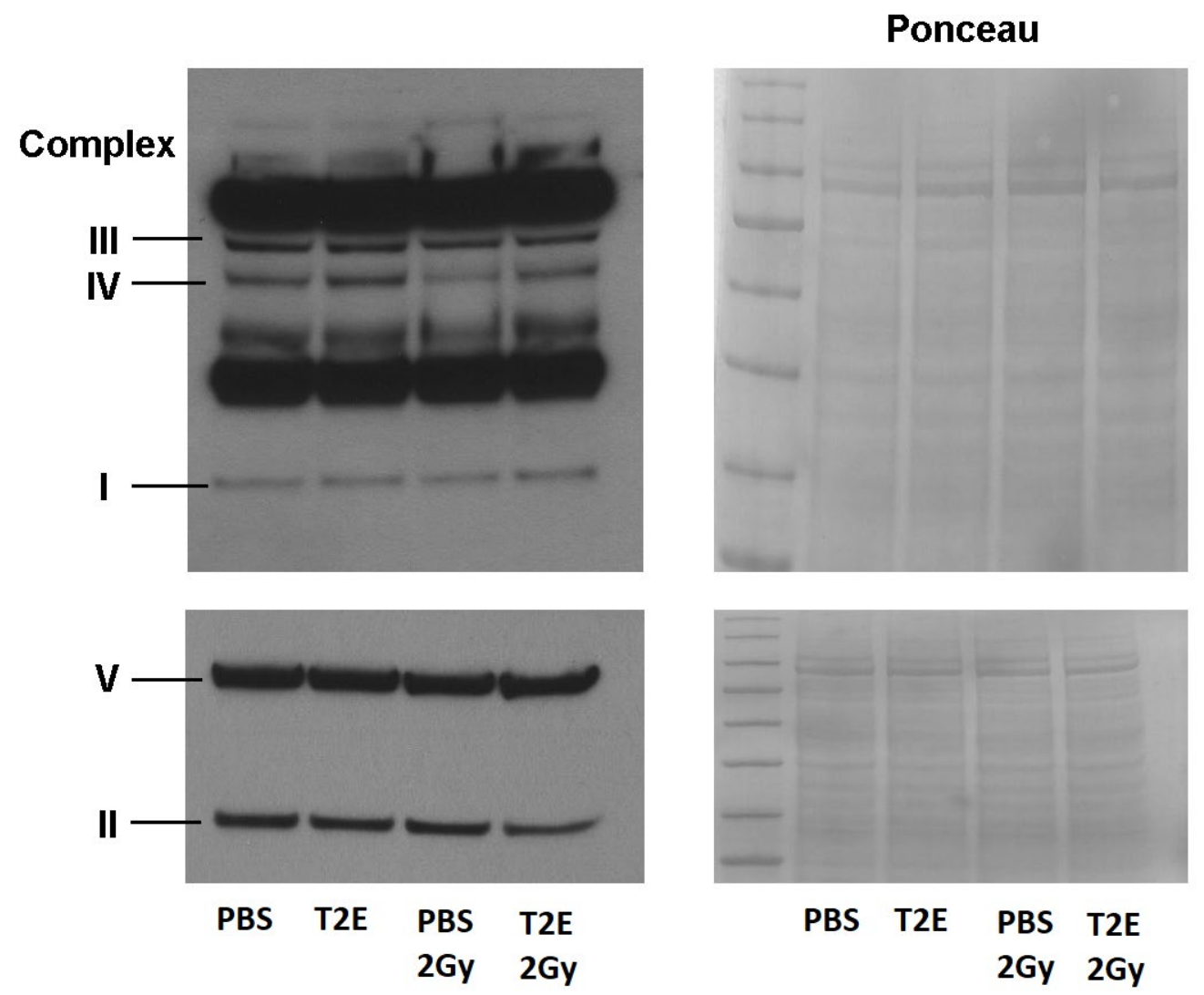
B
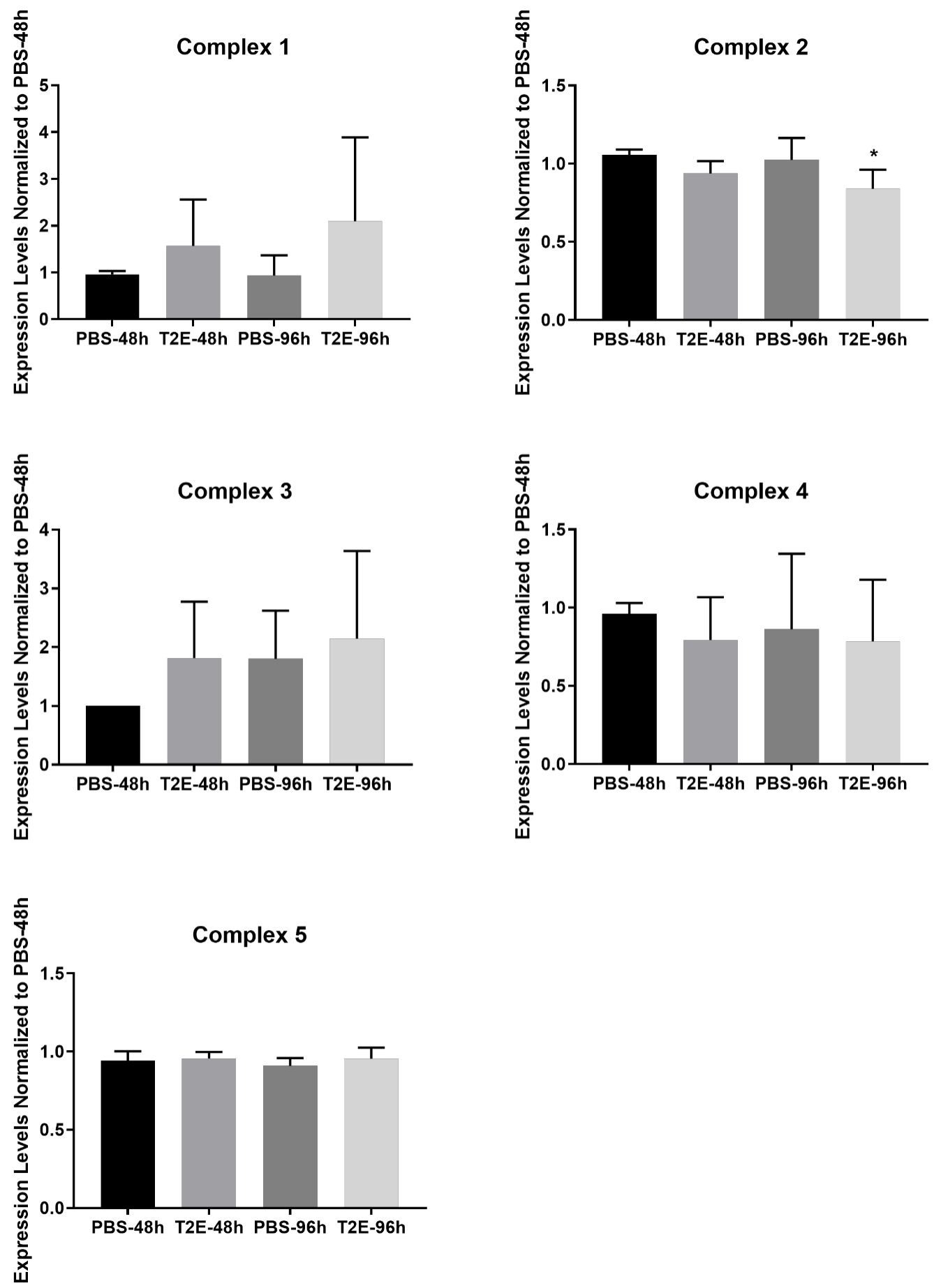
Figure 28. MnTE-2-PyP treatment altered PC3 cells metabolism in combination with radiation. Cells were seeded in media containing PBS or $30 \mu \mathrm{M}$ MnTE-2-PyP. After 24 hours, some groups of cells were exposed to 2 Gy radiation. After another 48 hours, one day before experiment, cells were re-seeded at a density of 25,000 cells/ well in a $96-$ well Seahorse plate and incubated at $37^{\circ} \mathrm{C}$ with $5 \% \quad \mathrm{CO}_{2}$ overnight. (A) OCR real-time measurements with the addition of oligomycin, FCCP, and rotenone/antimycin. (B) Calculation of non-mitochondrial respiration, basal respiration, maximal respiration, ATP production, proton leak, and spare respiratory capacity based on OCR curves. The data were normalized to total cell protein content as measured by Bradford assay. (C) ECAR real-time measurements with addition of glucose, oligomycin, and 2-deoxy-D-glucose. (D) Calculation of glycolysis, glycolytic capacity, glycolytic reserve, and non-glycolytic acidification based on ECAR curves. The data were normalized to total cell protein content as measured by Bradford assay. ${ }^{*} p<0.05$ compared to PBS treatment. 
A

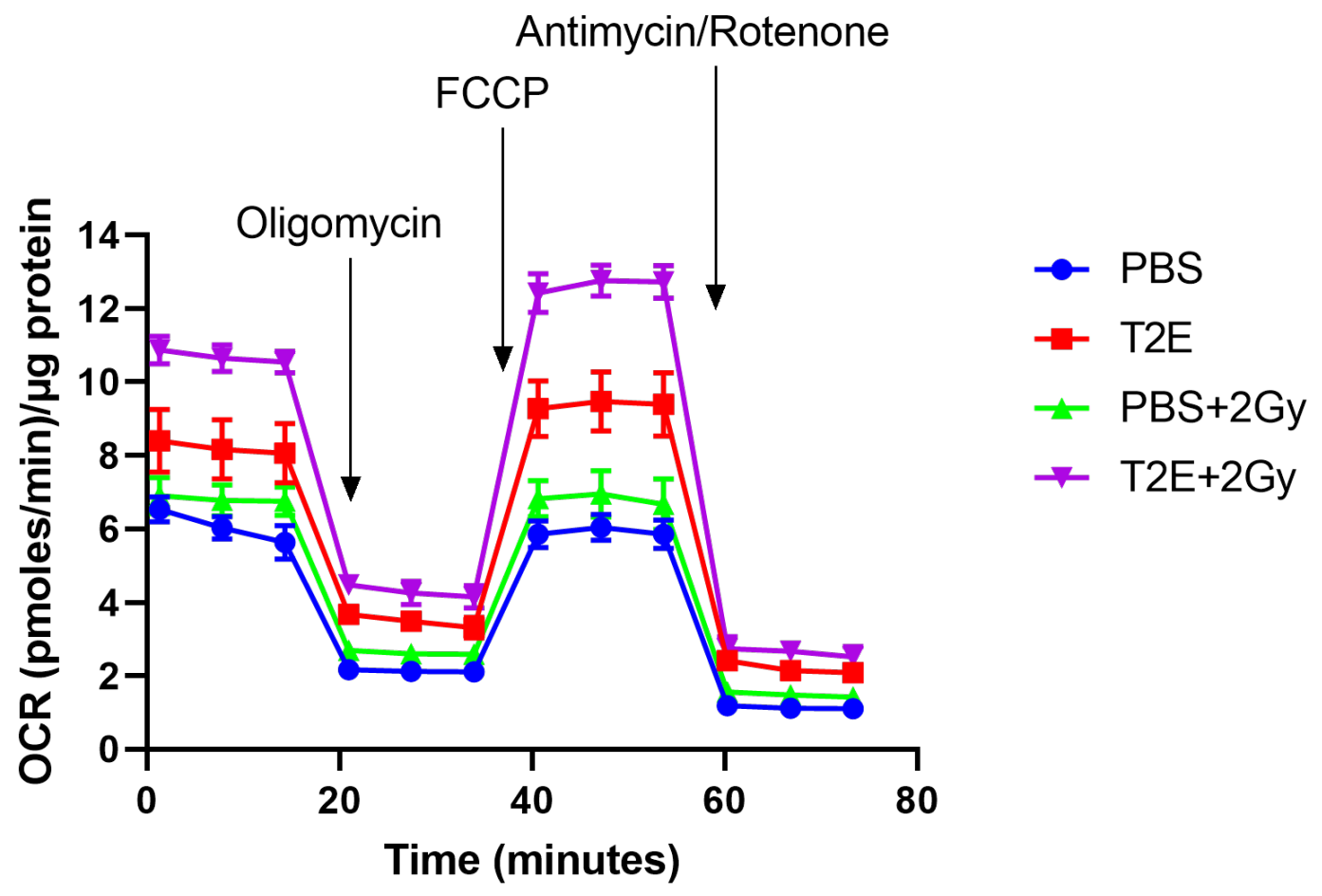


B
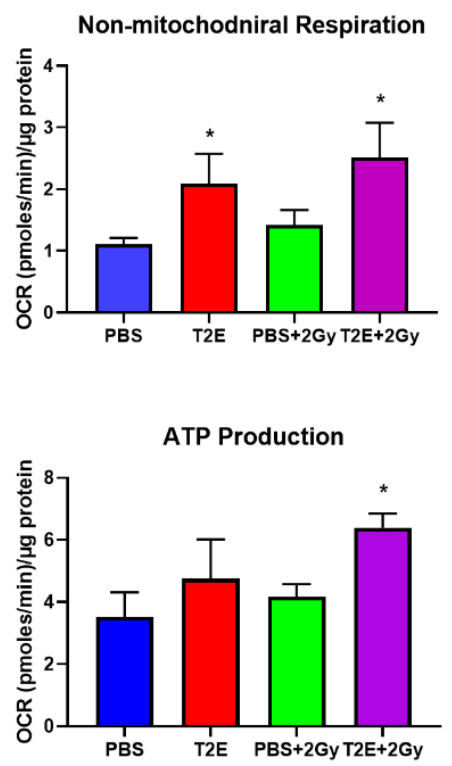
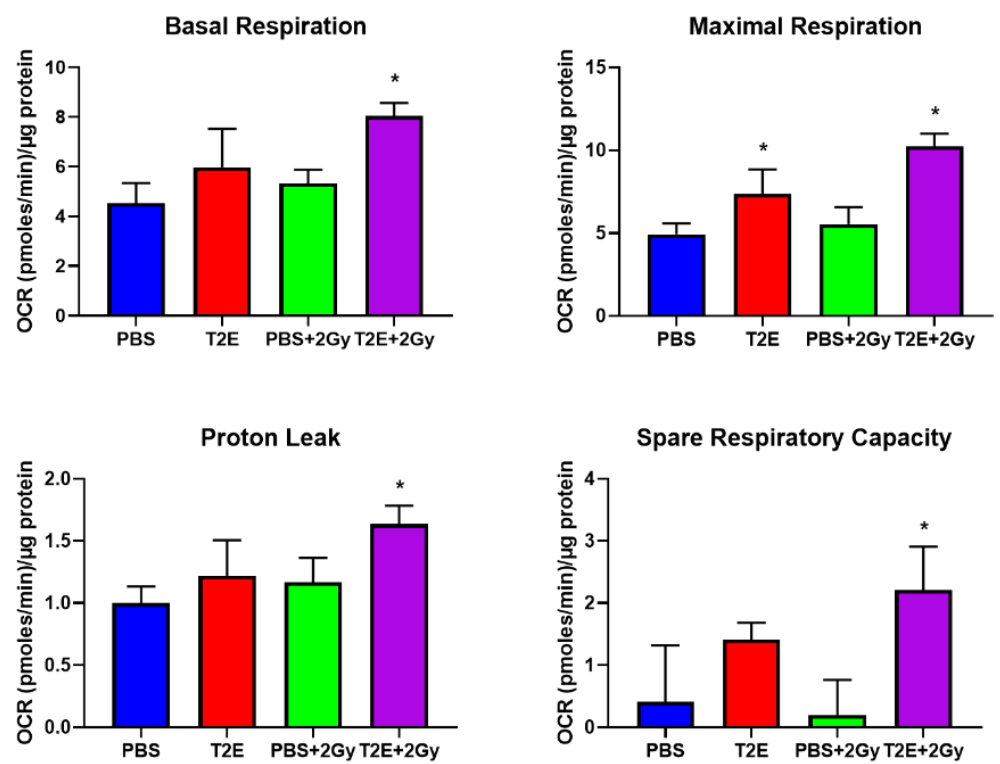
C

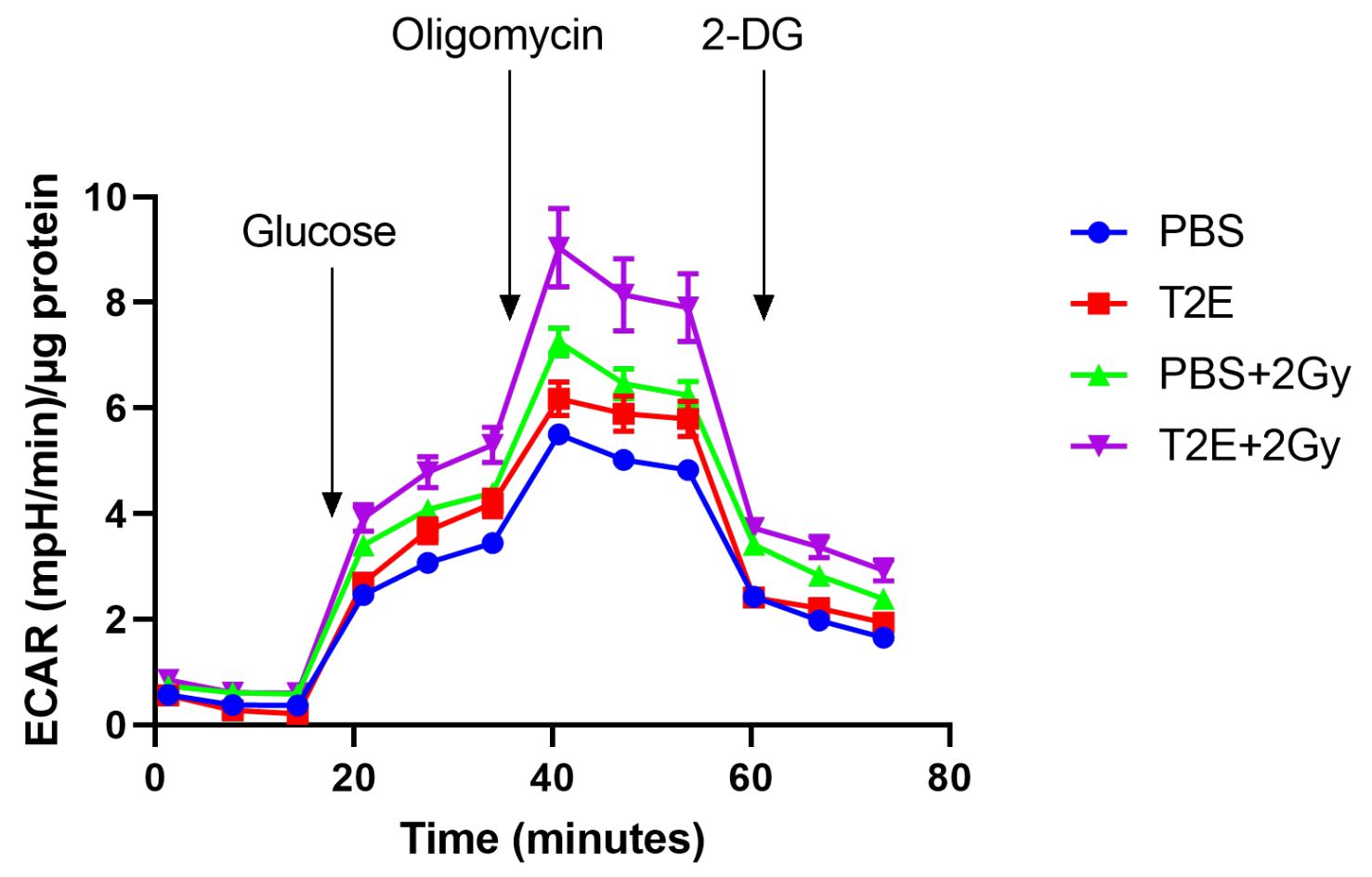


D
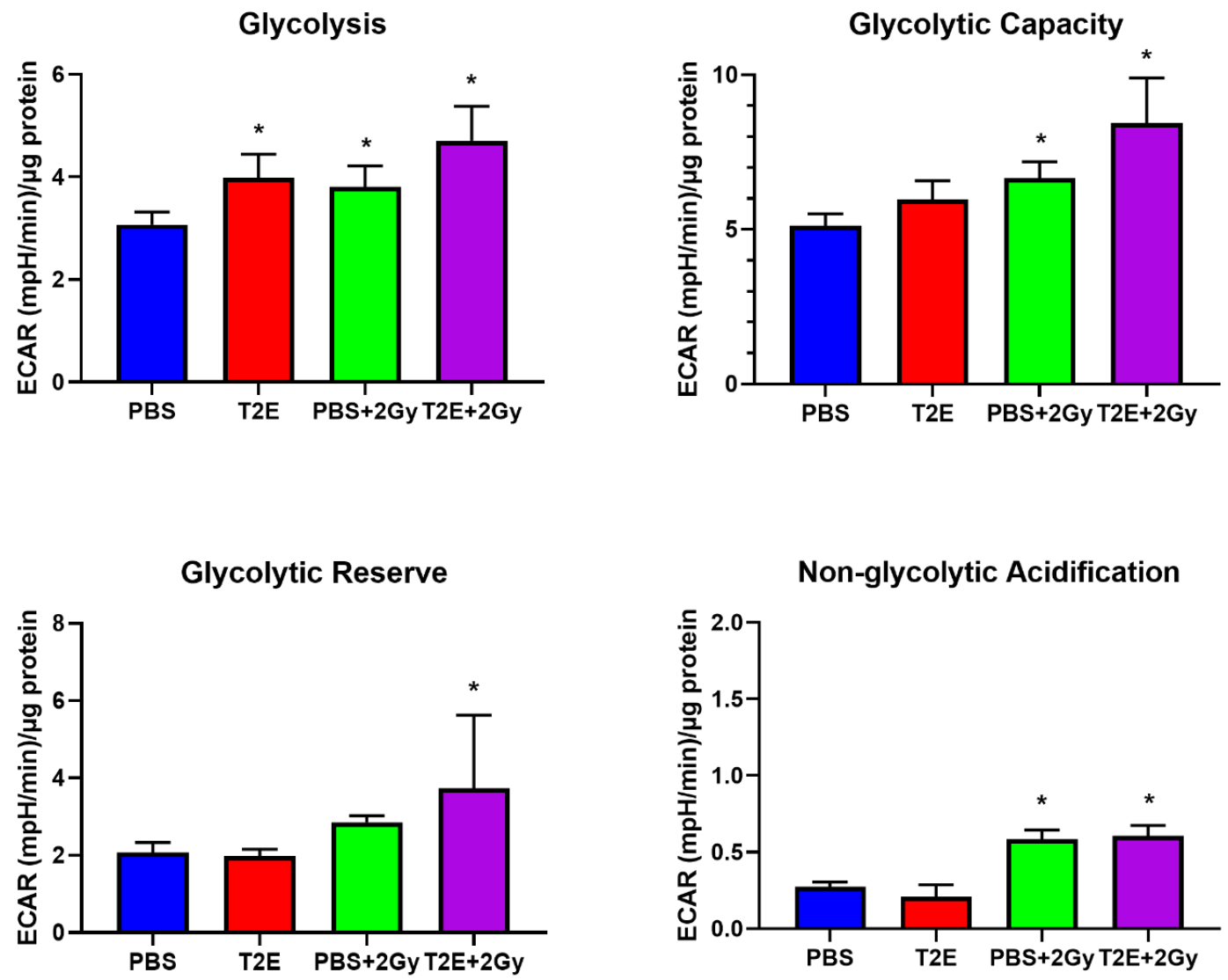
Figure 29. MnTE-2-PyP treatment altered LNCaP cells metabolism in combination with radiation. Cells were seeded in media containing PBS or $30 \mu \mathrm{M}$ MnTE-2-PyP. After 24 hours, some groups of cells were exposed to 2 Gy radiation. After another 48 hours, one day before experiment, cells were re-seeded at a density of 25,000 cells/ well in a $96-$ well Seahorse plate and incubated at $37^{\circ} \mathrm{C}$ with $5 \% \mathrm{CO}_{2}$ overnight. (A) OCR real-time measurements with addition of oligomycin, FCCP, and rotenone/antimycin. (B) Calculation of non-mitochondrial respiration, basal respiration, maximal respiration, ATP production, proton leak, and spare respiratory capacity based on OCR curves. The data were normalized to total cell protein content as measured by Bradford assay. (C) ECAR real-time measurements with addition of glucose, oligomycin, and 2-deoxy-D-glucose. (D) Calculation of glycolysis, glycolytic capacity, glycolytic reserve, and non-glycolytic acidification based on ECAR curves. The data were normalized to total cell protein content as measured by Bradford assay. ${ }^{*} p<0.05$ compared to PBS treatment. 
A

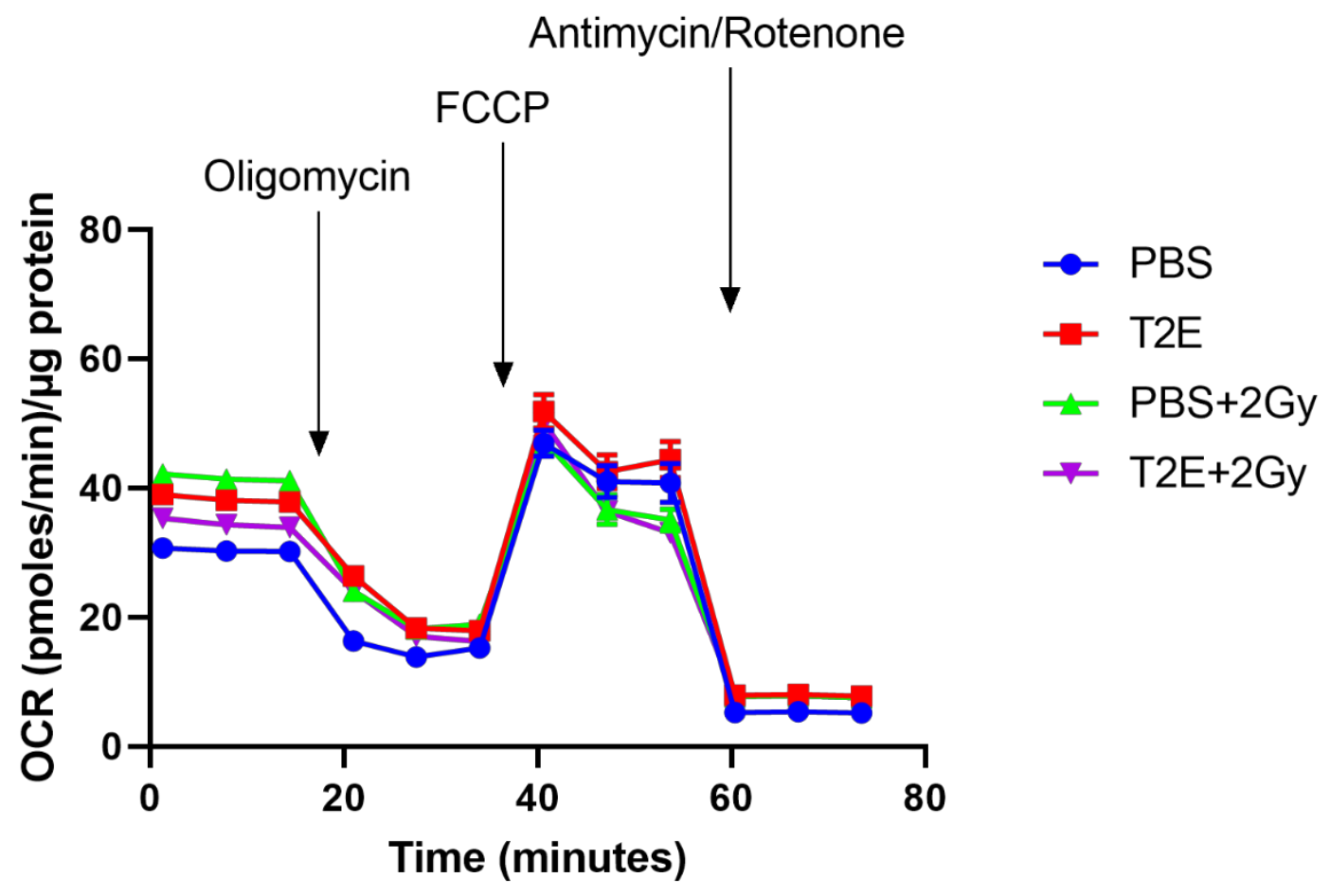


B
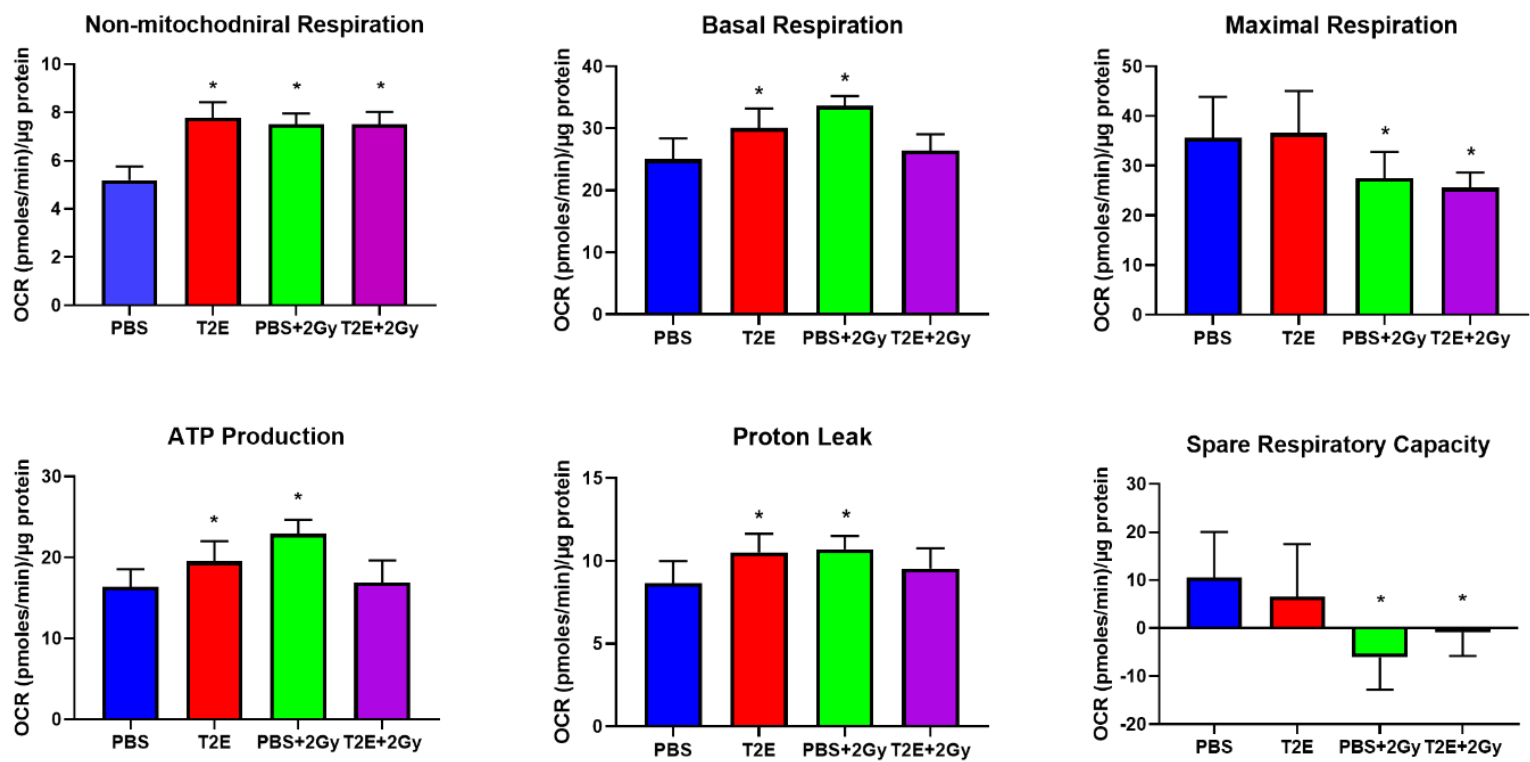
C

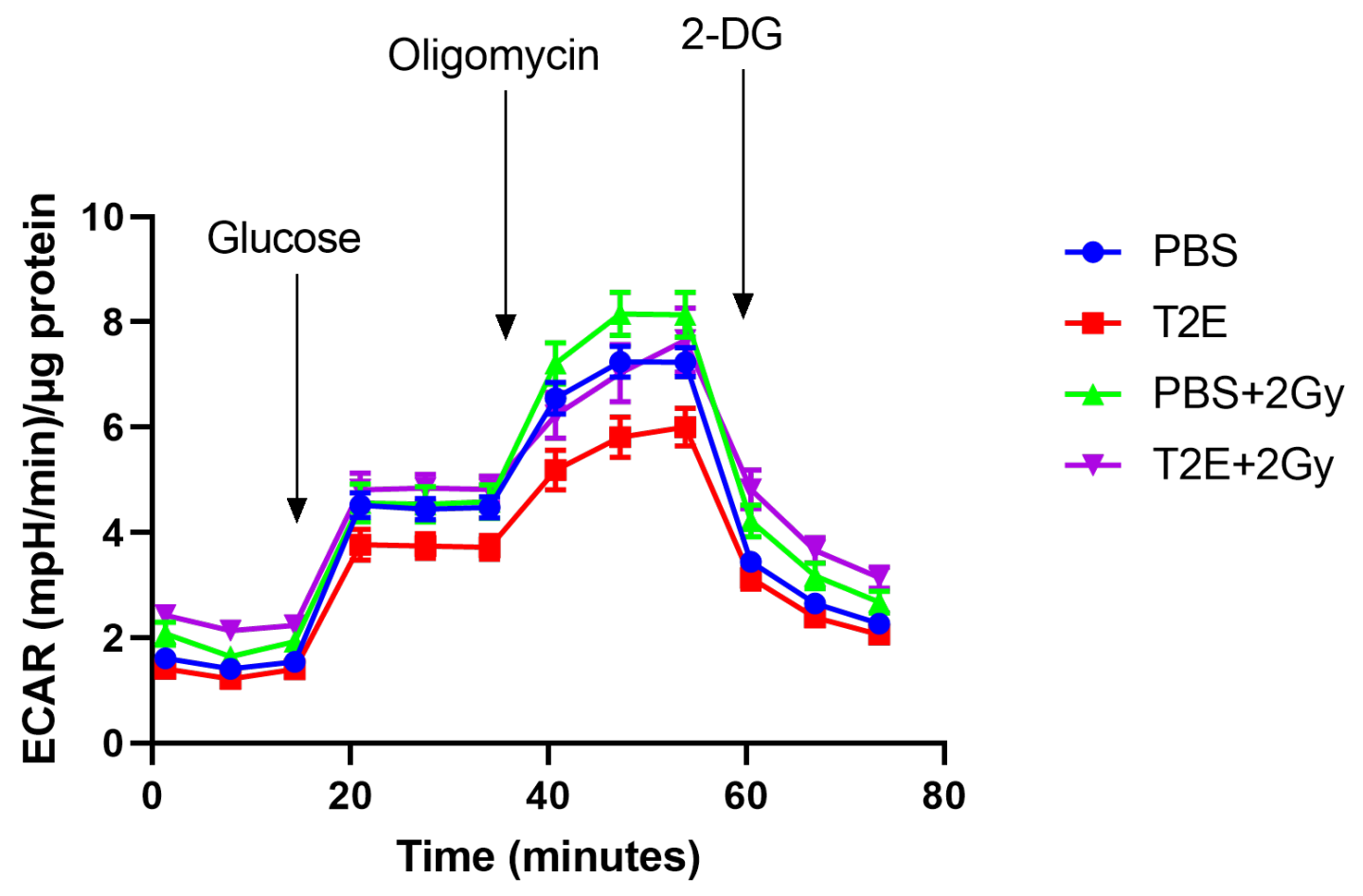


D
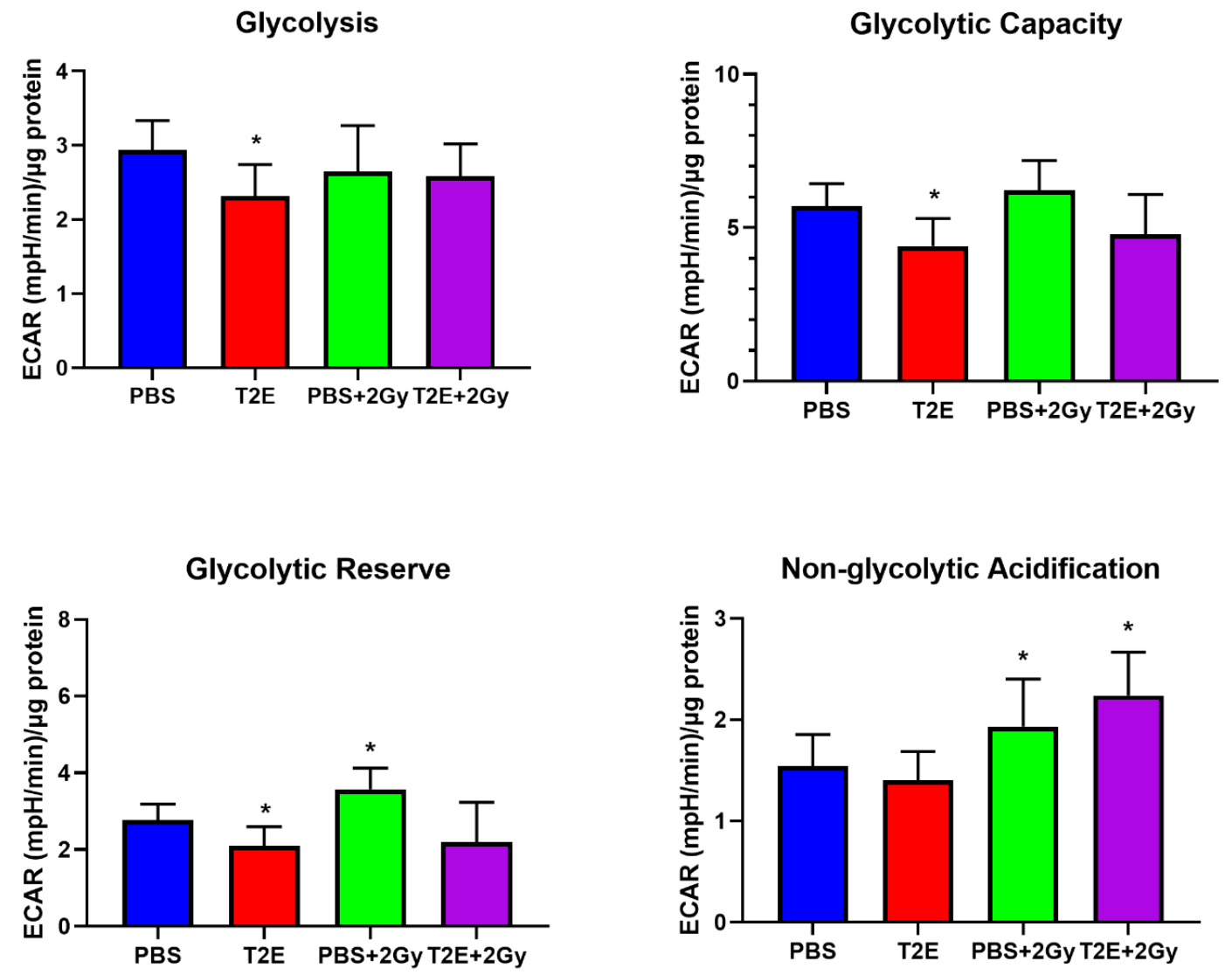


\subsection{MnTE-2-PyP and radiation treatment affect prostate cancer cell ATP levels and membrane potential}

Since OXPHOS, and glycolysis were affected by MnTE-2-PyP treatments, we measured actual cellular ATP levels to determine whether prostate cancer cells are still able to maintain the ATP balance (Figure 30A). We found that MnTE-2-PyP significantly decreased cellular ATP levels ( $\sim 35 \%$ decrease) as compared to other treatments in PC3 cells, but in LNCaP cells, MnTE-2-PyP had no effects on ATP levels and radiation significantly increased cellular ATP levels ( $35 \%$ increase).

To determine whether the mitochondrial membrane potential was affected by MnTE-2-PyP or radiation in prostate cancer cells, we measured the membrane potential in both PC3 and LNCaP cells using a TMRM probe. We found that only radiation slightly increased the membrane potential in LNCaP cells, which indicate there was no significant damage that may inhibit mitochondria function (Figure 30B). 
Figure 30. MnTE-2-PyP and radiation affects cellular ATP levels and radiation affects mitochondrial membrane potential in prostate cancer cells. PC3 and LNCaP cells were seeded in media containing PBS or $30 \mu \mathrm{M}$ MnTE-2-PyP. After 24 hours, some groups of cells were exposed to 2 Gy radiation. After another 72 hours, (A) Cellular ATP levels were measured by an ATP assay kit. (B) Mitochondrial membrane potential was measured by TMRM probes via flow cytometry analysis. All data represent mean \pm SD from at least three independent experiments. ${ }^{*} p<0.05$ compared to PBS treatment. 
A

PC3

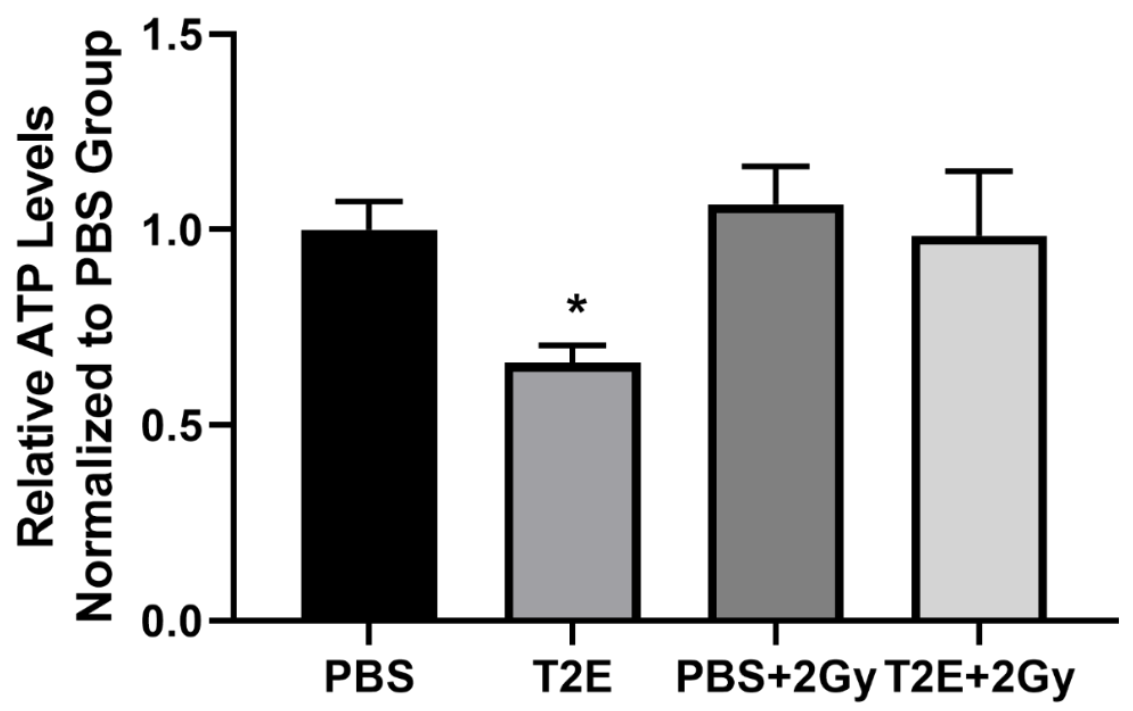

LNCaP

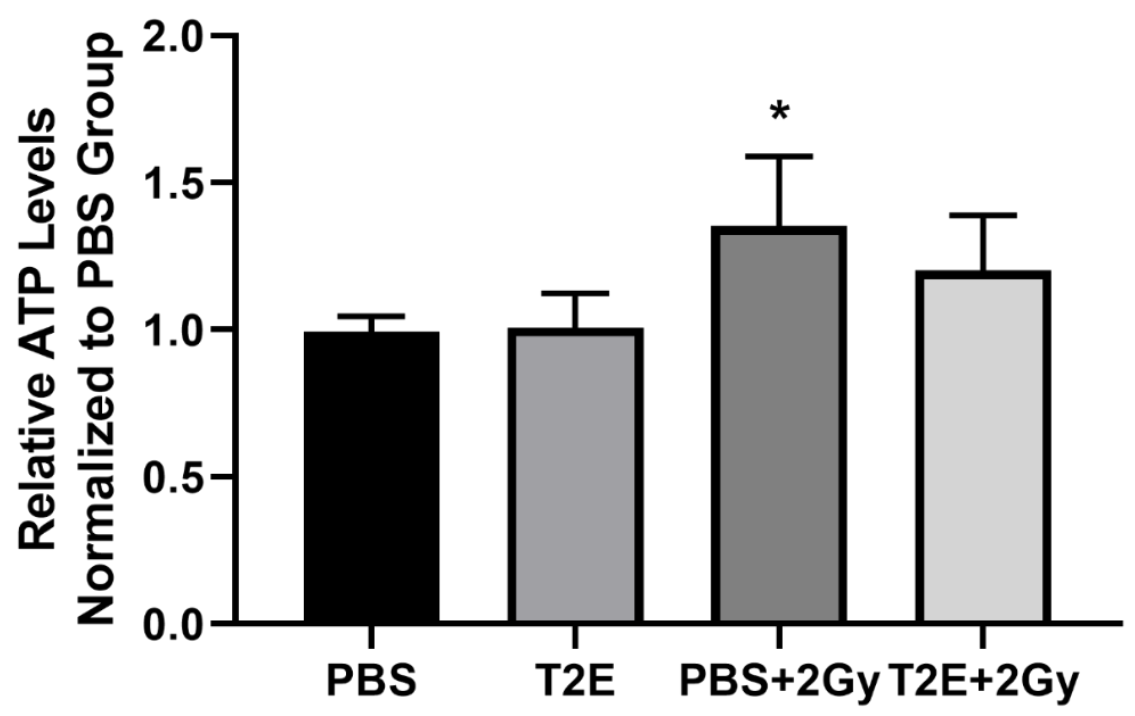


B

\section{PC3}

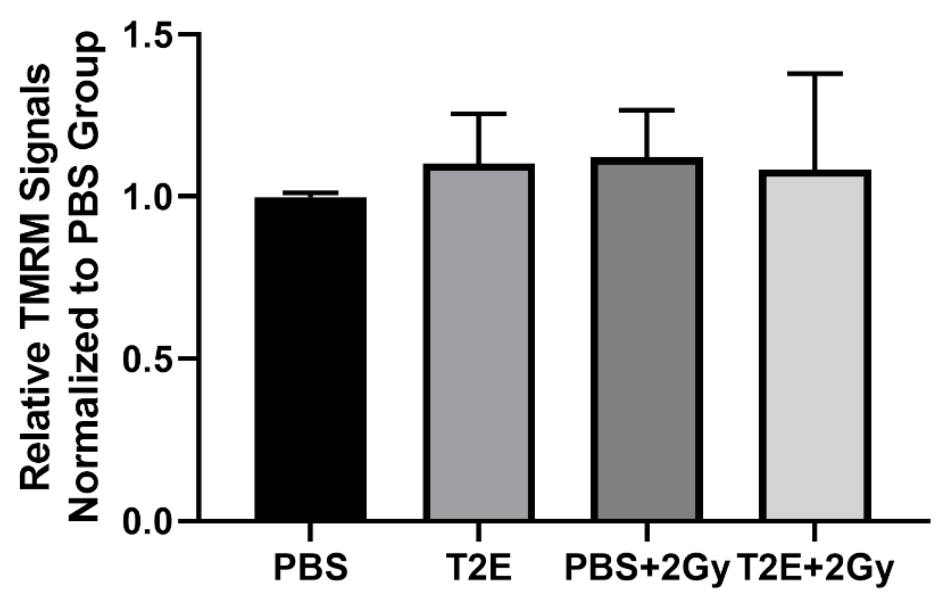

LNCaP

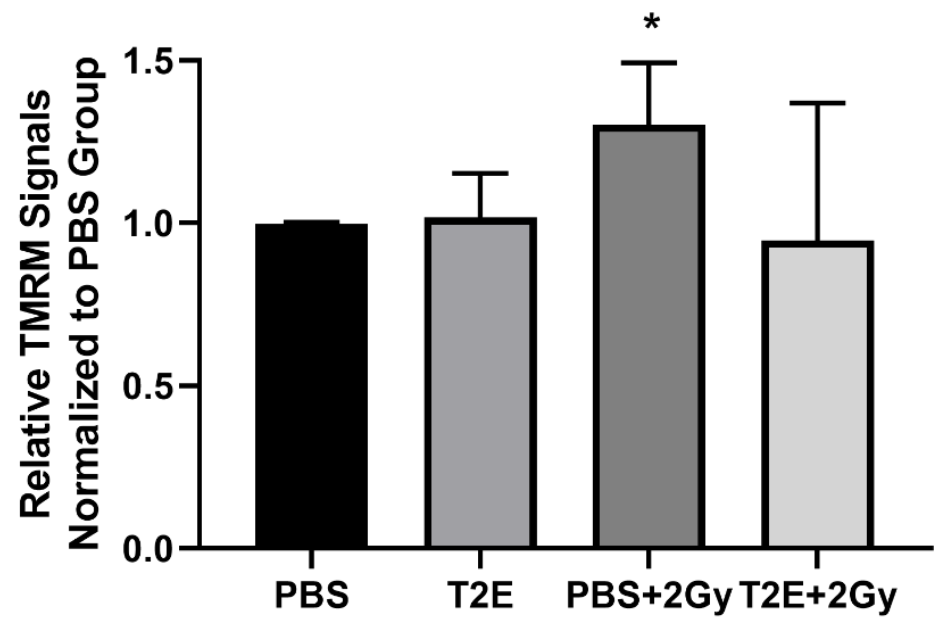




\subsection{The effects of MnTE-2-PyP on prostate cancer cell glucose uptake}

Based on the Seahorse results, we found that MnTE-2-PyP had a significant impact on prostate cancer cell energy metabolism. In addition, MnTE-2-PyP and radiation had different combinatorial effects on PC3 and LNCaP cells. In order to determine the cause of such alterations in energy metabolism, we measured the glucose uptake in PC3 and LNCaP cells by using a fluorescent analogue, 2-NBDG.

The 96 hours treatments period showed that MnTE-2-PyP or radiation or the combination could lead to an increase of glucose uptake (Figure 31A). We also investigated whether MnTE-2-PyP has an acute influence by measuring glucose uptake after 6 hours treatment. Although there is an trend of increased glucose uptake in both cell lines, only MnTE-2-PyP-treated PC3 cells showed a significant difference as compared to PBS-treated one (Figure 31B). These results indicate that MnTE-2-PyP directly induces glucose uptake in prostate cancer cells. 
Figure 31. MnTE-2-PyP and radiation alone or in combination enhanced glucose uptake in prostate cancer cells. (A) PC3 and LNCaP cells were seeded in media containing PBS or $30 \mu \mathrm{M}$ MnTE-2-PyP. After 24 hours, some groups of cells were exposed to 2 Gy radiation. After another 72 hours, 600,000 cells were resuspended in $1.2 \mathrm{ml}$ of media containing $2 \mu \mathrm{M}$ 2-NBDG for 30 min at $37{ }^{\circ} \mathrm{C}$. Cells were washed with PBS and resuspended in media for FACS analysis. (B) PC3 and LNCaP cells were treated with PBS or $30 \mu \mathrm{M}$ MnTE-2-PyP for 6 hours. Then 600,000 cells were resuspended in $1.2 \mathrm{ml}$ of media containing $2 \mu \mathrm{M}$ 2-NBDG for $30 \mathrm{~min}$ at $37^{\circ} \mathrm{C}$. Cells were washed with PBS and resuspended in media for FACS analysis. All data represent mean \pm SD from at least three independent experiments. ${ }^{*} p<0.05$ compared to PBS treatment. $\# p<0.05$ compared to PBS + 2Gy treatment. 
A

\section{6 hours}
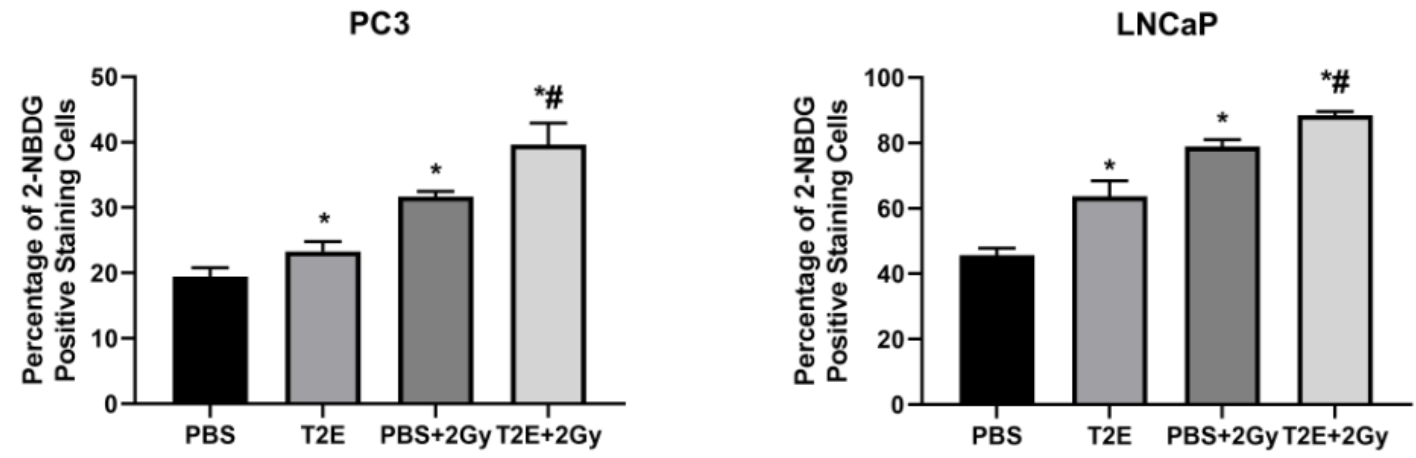

B

\section{6 hours}
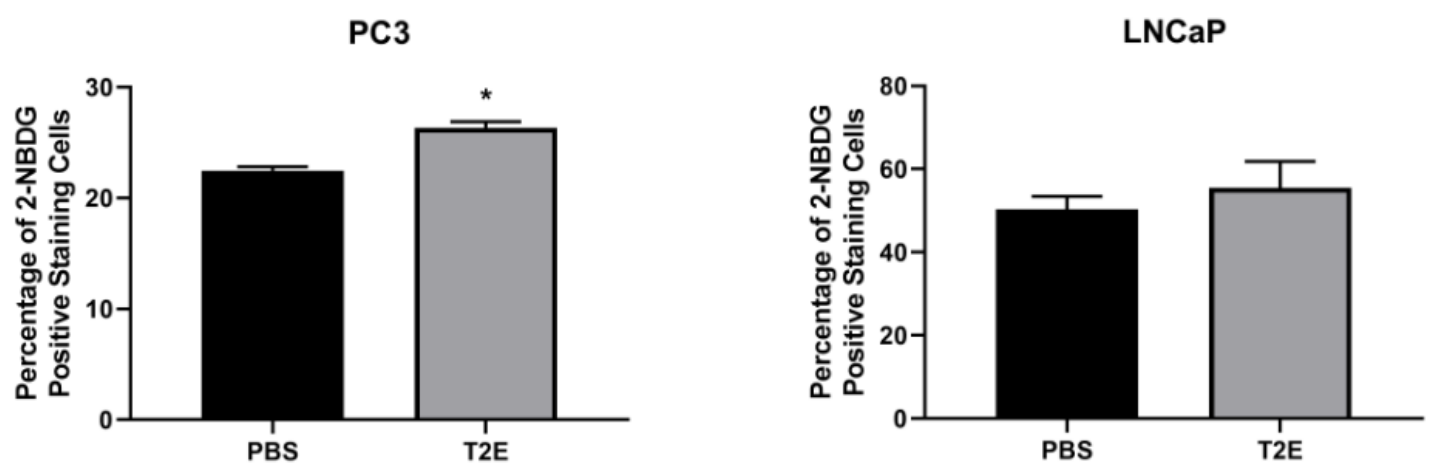


\subsection{The effects of MnTE-2-PyP on NAD(H) and NADP(H) levels in prostate cancer cells}

$\mathrm{NADH}$ is the central molecule involved in OXPHOS. Based on the altered metabolism patterns in prostate cancer cells that was identified by the Seahorse analysis, we further investigated whether MnTE-2-PyP and radiation have an impact on the NAD $(H)$ pool, which can potentially contribute to OXPHOS rate alterations. We found that MnTE2-PyP alone, or combined with radiation significantly increased the NAD+/NADH ratios in both PC3 and LNCaP cells (Figure 32A). Decreased NADH levels were identified in these two cell lines, as the major contributor to the increased NAD+/NADH ratios (Figure 32). Although NAD+/NADH ratios were not altered in PC3 cells treated with radiation, $\mathrm{LNCaP}$

cells displayed higher NAD+ and NADH levels after radiation treatment, but the $\mathrm{NAD+} / \mathrm{NADH}$ ratio remained the same as compared to PBS treatment (Figure 32C). These data indicate that MnTE-2-PyP had similar effects on the NAD+ and NADH pools in both PC3 and LNCaP cells, but LNCaP cells showed an additional response to radiation.

The NADP+/NADPH redox couple plays an important role in cellular antioxidant defense [125]. We are interested in cellular NADPH pools of MnTE-2-PyP-treated prostate cancer cells after observing significant decrease of NADH pools. Similar to the increased $\mathrm{NAD+/NADH}$ ratios in MnTE-2-PyP-treated cells, we found that MnTE-2-PyP greatly enhanced NADP+/NADPH ratios in both prostate cancer cell lines, $\sim 40$ fold increase in LNCaP cells (Figure 33). We also measured the NADP+ and NADPH levels in cells and identified that increased NADP+ and decreased NADPH levels together result in the huge change in NADP+/NADPH ratios. In addition, LNCaP cells showed a response to radiation treatment, where an increase in NADP+ levels was observed after 2 Gy of radiation (Figure 33C). Interestingly, we confirmed a dose-dependent effect of both MnTE-2-PyP and MnTnBuOE-2-PyP on the NADPH pool in PC3 cells (Figure 34). These data indicate 
that Mn porphyrins treatment deplete the cellular NADPH pool and may affect antioxidantdefense system.

To further validate the $N A D(P)+/ N A D(P) H$ levels results acquired from company assay kits, we also performed HPLC on prostate cancer cells with MnTE-2-PyP treatment to measure $\mathrm{NAD}(\mathrm{P})+/ \mathrm{NAD}(\mathrm{P}) \mathrm{H}$ levels. Consistently, we found that MnTE-2-PyP treatment depleted NADPH pools in both PC3 ( 62\% decrease) and LNCaP ( 42\% decrease) cells and significantly increased the NADP+/NADPH ratios, but MnTE-2-PyP also reduced NADP+ levels in PC3 cells ( $25 \%$ decrease), which was opposite to what we observed from the commercial assay kit results (Figure 35). In addition, MnTE-2-PyP significantly enhanced the NAD+/NADH ratios in PC3 cells, but in LNCaP cells, the increase was not significantly different, which could be due to large variation. We are interested whether $\mathrm{FAD} / \mathrm{FADH} \mathrm{H}_{2}$ and succinate levels were altered in prostate cancer cells with MnTE-2-PyP, because these reductants contributes to the function of complex II of ETC. As for PC3 cells, there is no difference of $\mathrm{FAD} / \mathrm{FADH}_{2}$ levels between PBS and MnTE-2-PyP treatment (Figure 36A), but the succinate levels were significantly increased by MnTE-2PyP treatment ( $\sim 90 \%$ increase, Figure $36 \mathrm{C})$; for LNCaP cells, although it seems that there is a trend of decease of FADH 2 levels in MnTE-2-PyP-treated LNCaP cells, the difference was not significant (Figure 36B). In addition, the succinate level was not altered by MnTE2-PyP in LNCaP cells (Figure 36C).

Based on both assay kits and HPLC results, we confirmed that MnTE-2-PyP depleted NADPH pools in both PC3 and LNCaP cells, and we believed that MnTE-2-PyP also reduced NADH levels in both cell lines, though large variation exists in LNCaP samples. MnTE-2-PyP also affected other cellular reducing metabolites levels, but the effects are not consistent between PC3 and LNCaP cells. 
Figure 32. MnTE-2-PyP and radiation enhanced NAD+/NADH ratios in prostate cancer cells. PC3 and LNCaP cells were seeded in media containing PBS or $30 \mu \mathrm{M}$ MnTE-2-PyP. After 24 hours, some groups of cells were exposed to 2 Gy radiation. After another 72 hours, cellular NADH and NAD+ levels were determine by the NAD+/NADHGlo assay. (A) NAD+/NADH ratios in PC3 and LNCaP cells with different treatments. (B) NAD+ and NADH levels in PC3 cells. (C) NAD+ and NADH levels in LNCaP cells. All data represent mean $\pm S D$ from at least three independent experiments. ${ }^{*} p<0.05$ compared to PBS treatment. 
A

PC3

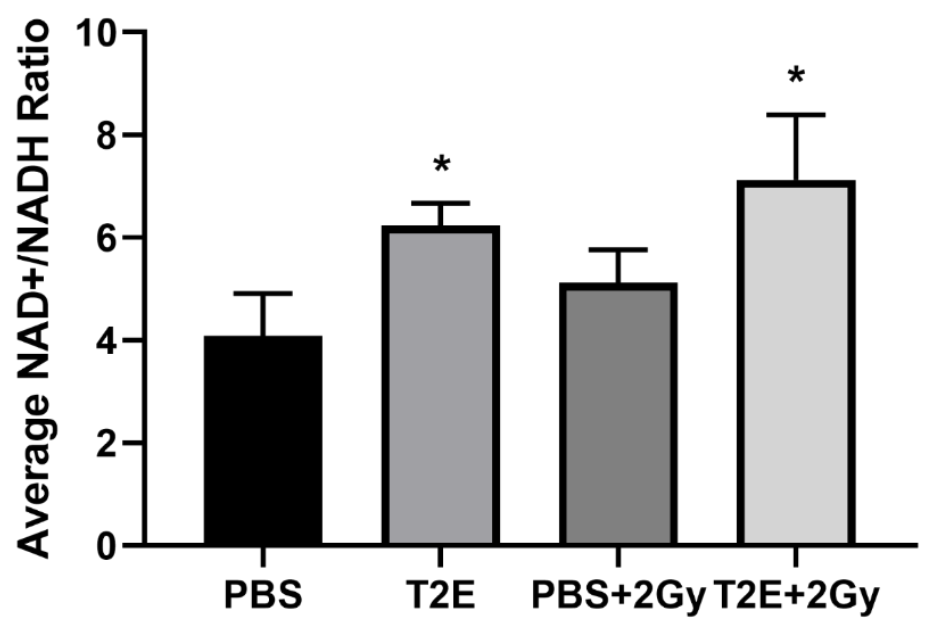

LNCaP

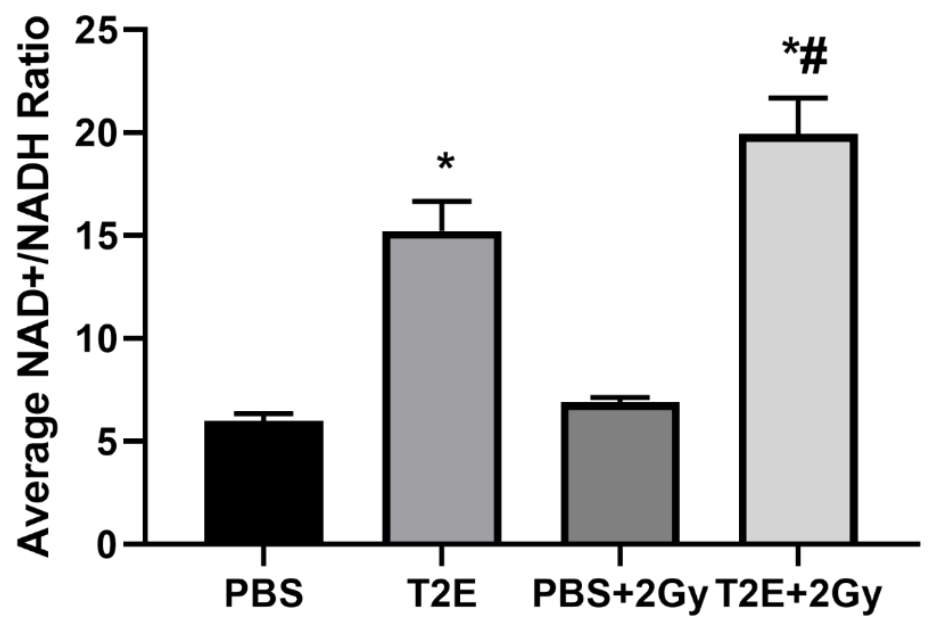


B

PC3-NAD+ levels

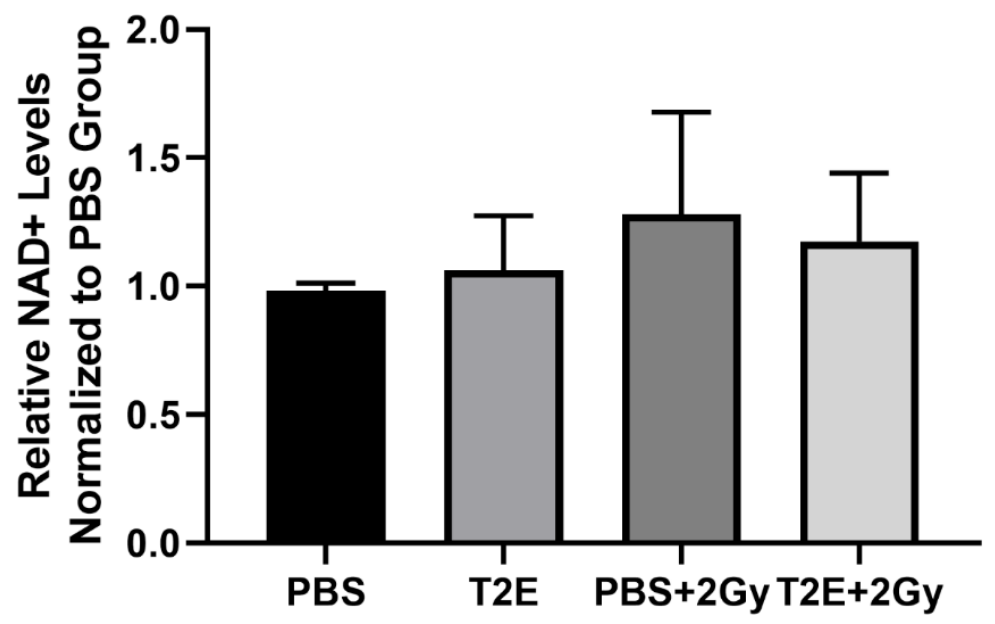

PC3-NADH levels

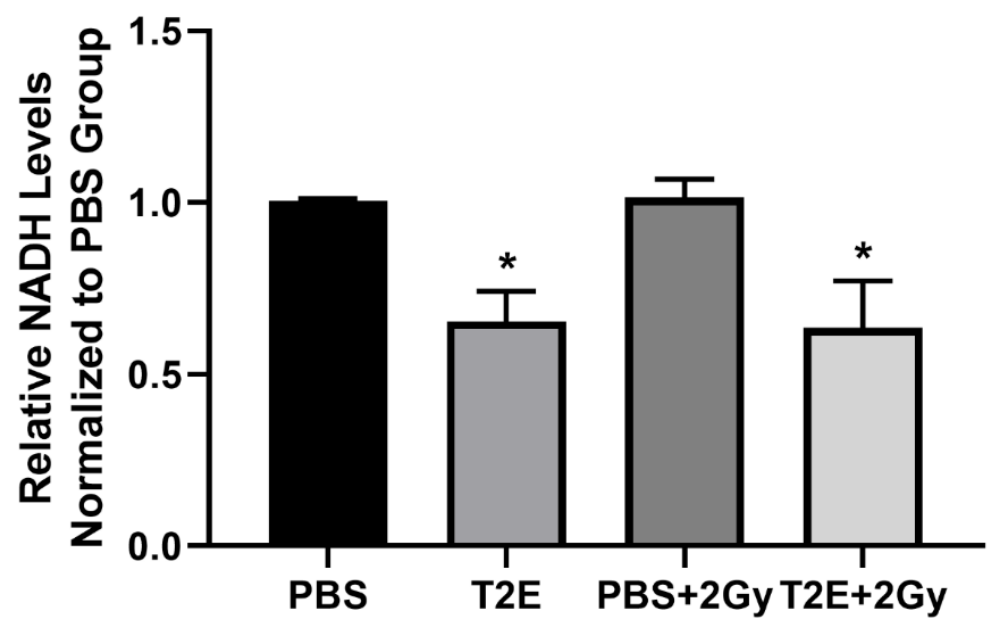


C

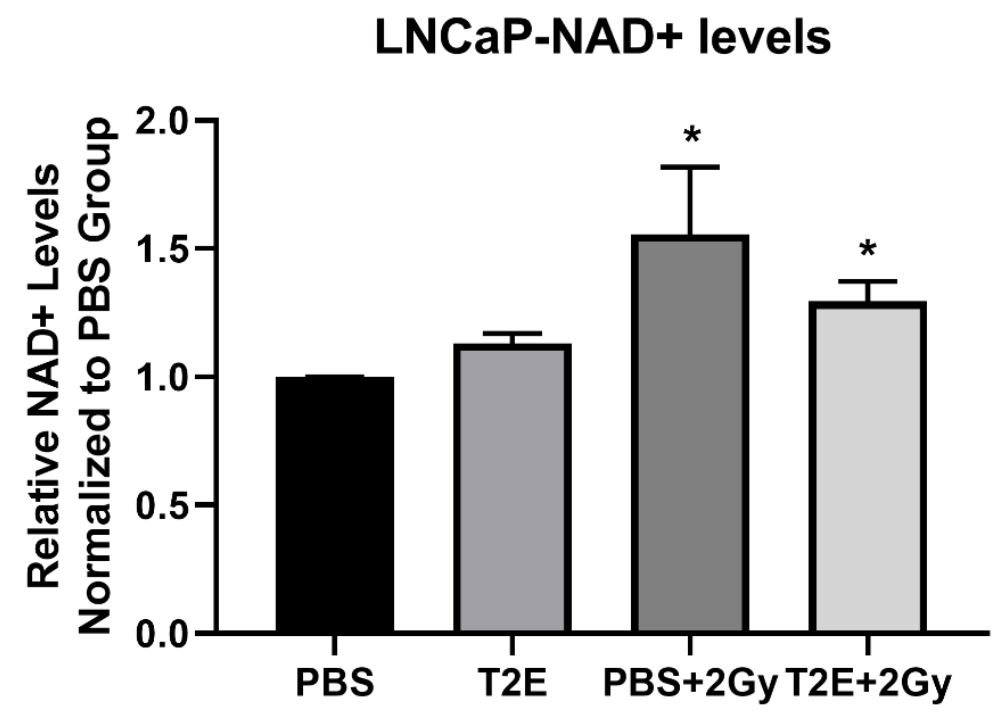

LNCaP-NADH levels

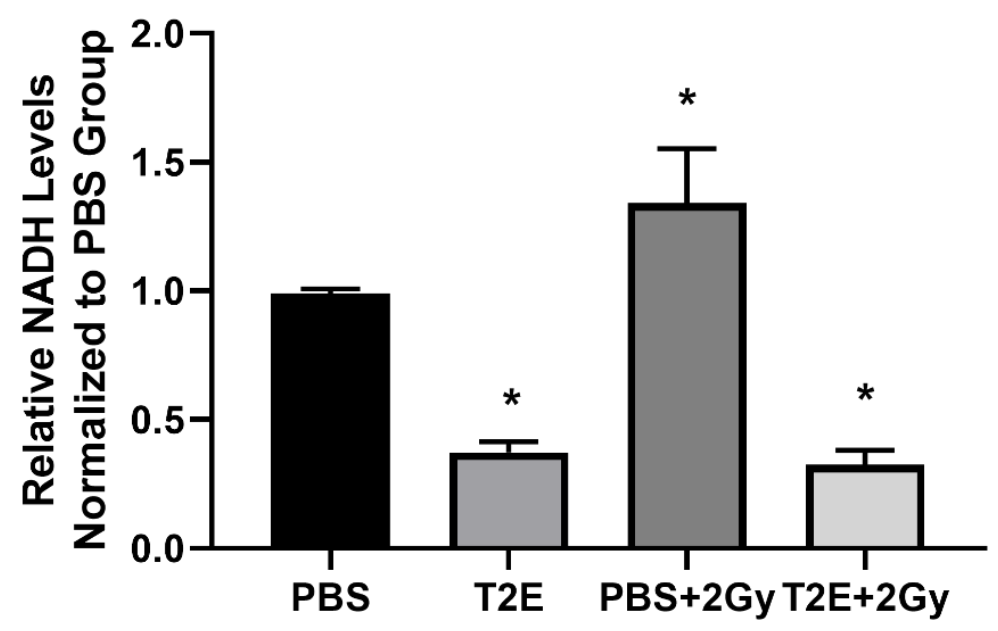


Figure 33. MnTE-2-PyP and radiation enhanced NADP+ levels and depleted NADPH pool in prostate cancer cells. PC3 and LNCaP cells were seeded in media containing PBS or $30 \mu \mathrm{M}$ MnTE-2-PyP. After 24 hours, some groups of cells were exposed to 2 Gy radiation. After another 72 hours, cellular NADPH and NADP+ levels were determine by the NADP+/NADPH-Glo assay. (A) NADP+/NADPH ratios in PC3 and LNCaP. (B) NADP+ and NADPH levels in PC3 cells. (C) NADP+ and NADPH levels in LNCaP cells with different treatments. All data represent mean $\pm S D$ from at least three independent experiments. ${ }^{*} p<0.05$ compared to PBS treatment. 
A
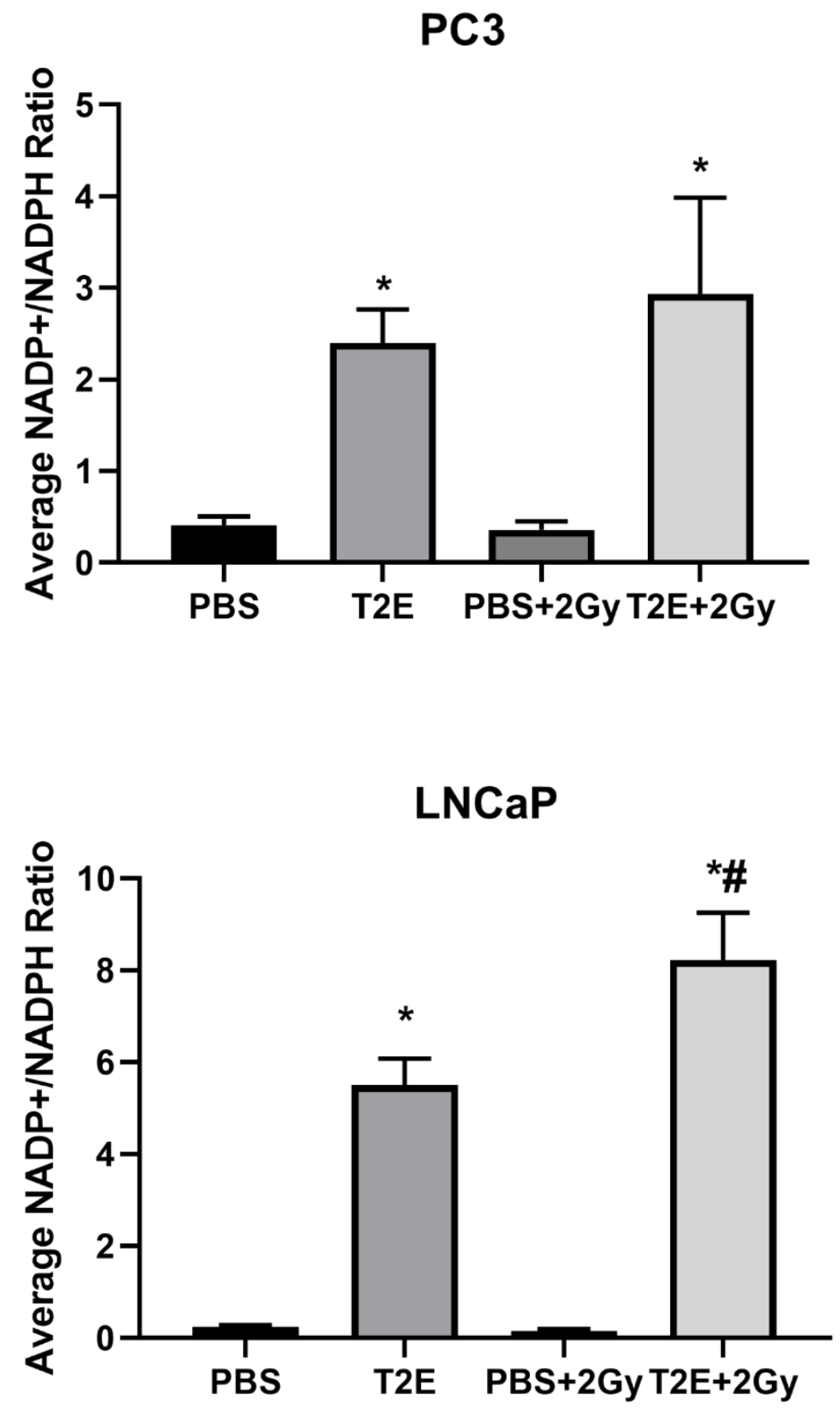
B

PC3-NADP+ levels

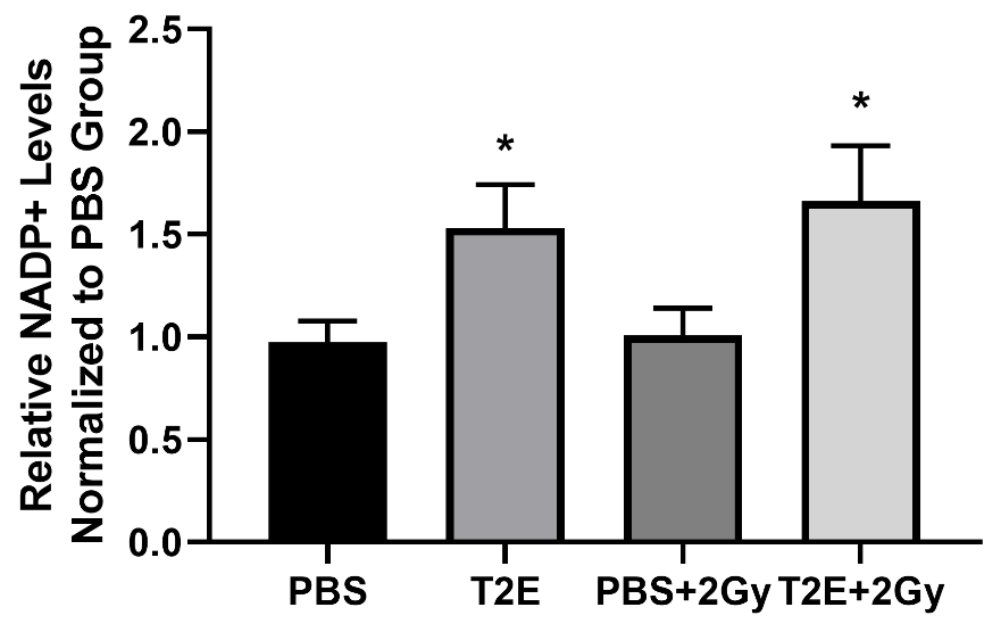

PC3-NADPH levels

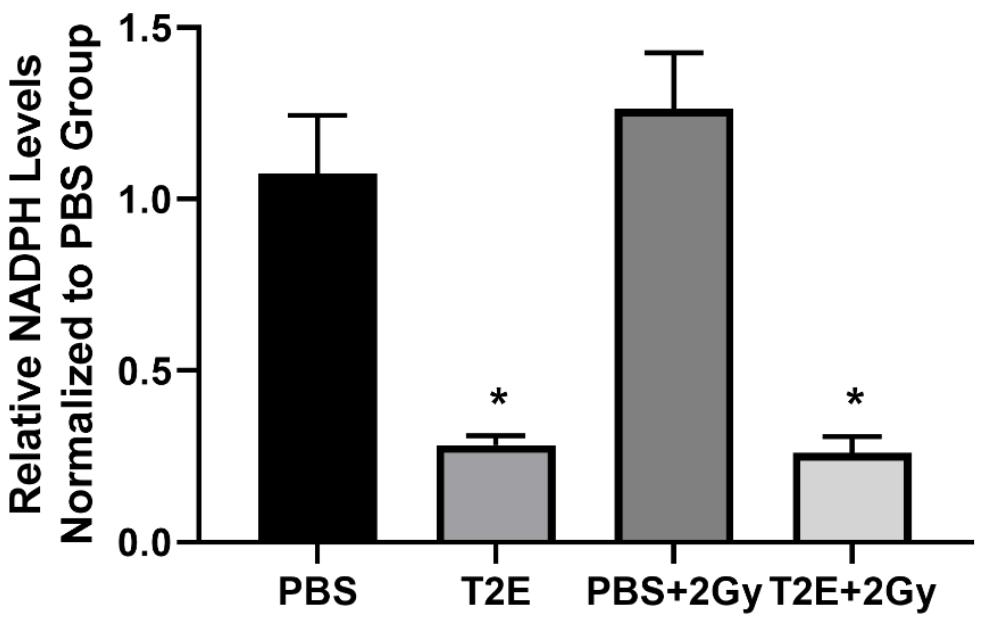


C

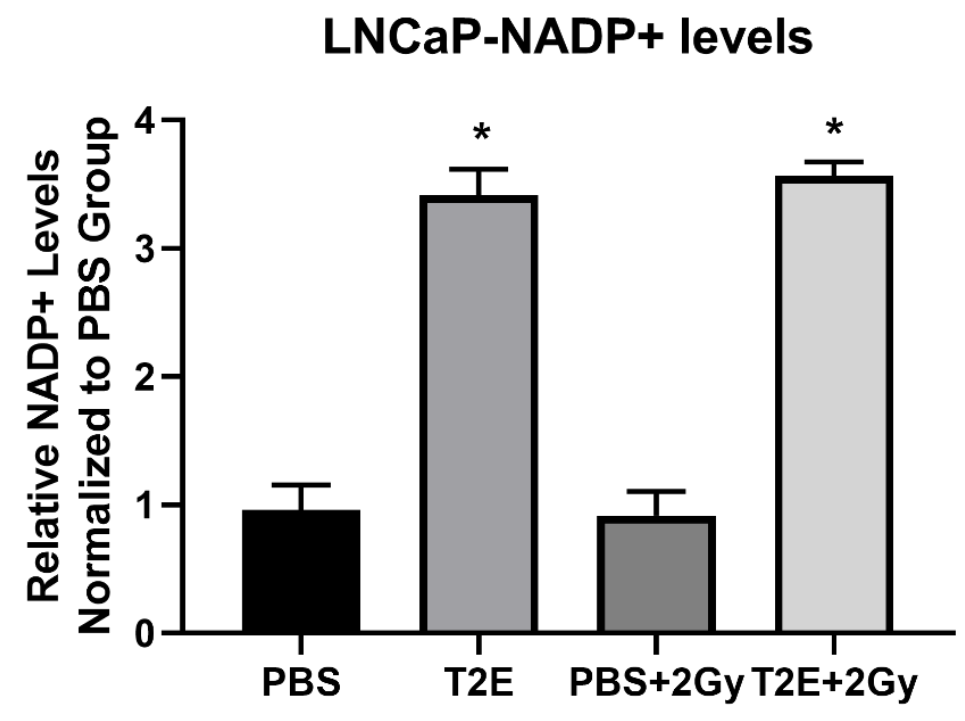

LNCaP-NADPH levels

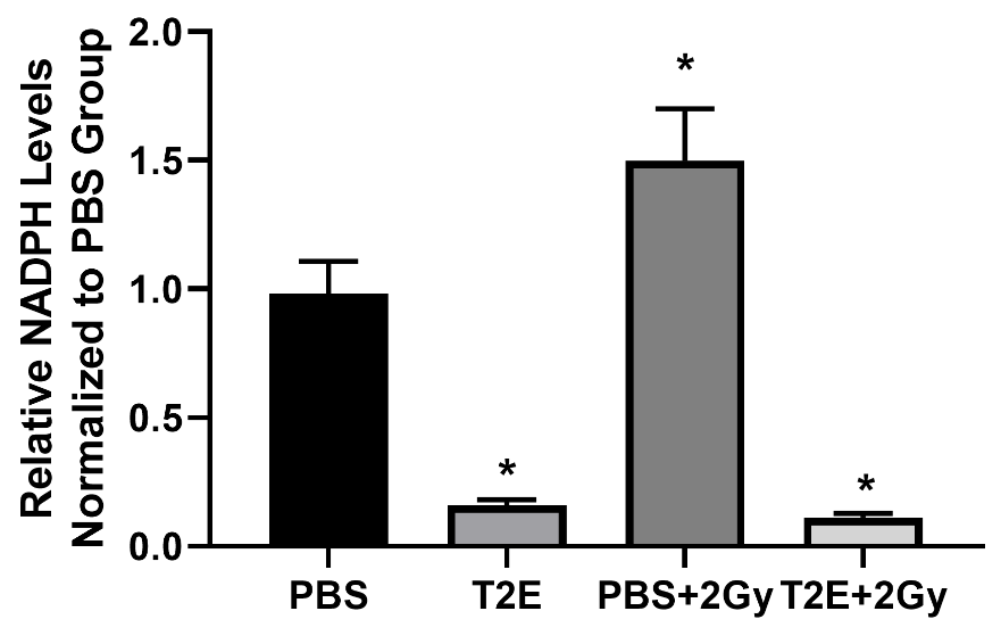


Figure 34. MnTE-2-PyP and MnTnBuOE-2-PyP affected NADP+/NADPH levels in a dose-dependent manner. PC3 cells were seeded in media containing PBS or 1, 5, 10 $\mu \mathrm{M}$ MnTE-2-PyP or MnTnBuOE-2-PyP. After 24 hours, some groups of cells were exposed to 2 Gy radiation. After another 72 hours, cellular NADPH and NADP+ levels were determine by the NADP+/NADPH-Glo assay. All data represent mean \pm SD from at least three independent experiments. ${ }^{*} p<0.05$ compared to PBS treatment. 


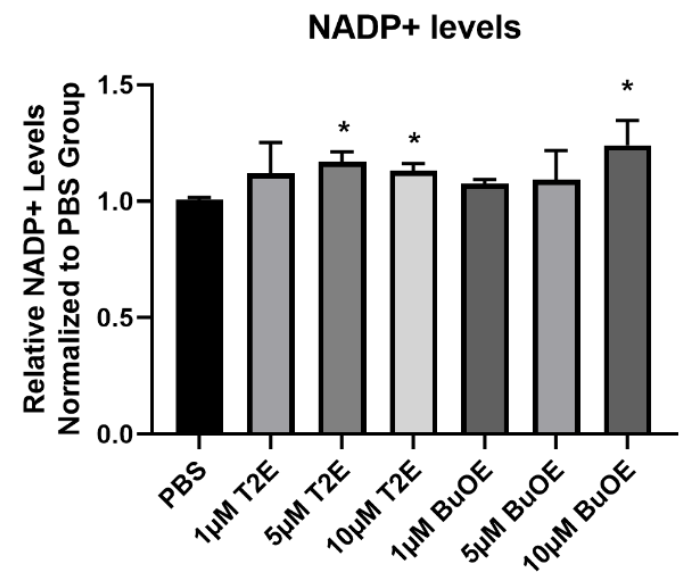

NADPH levels

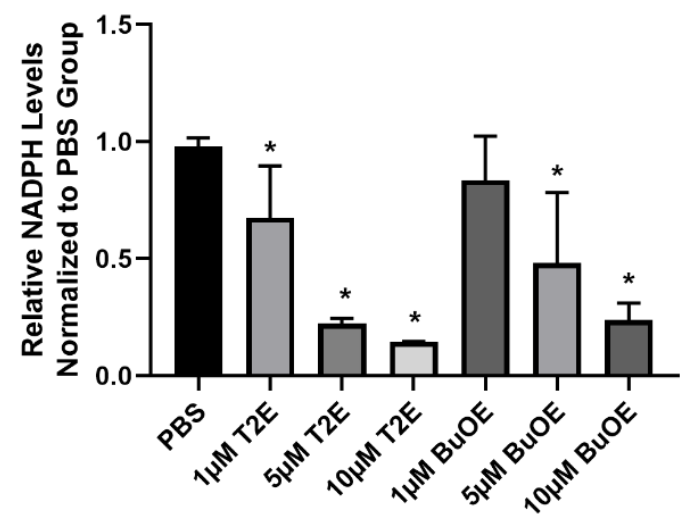

NADPH/NADP+ ratio

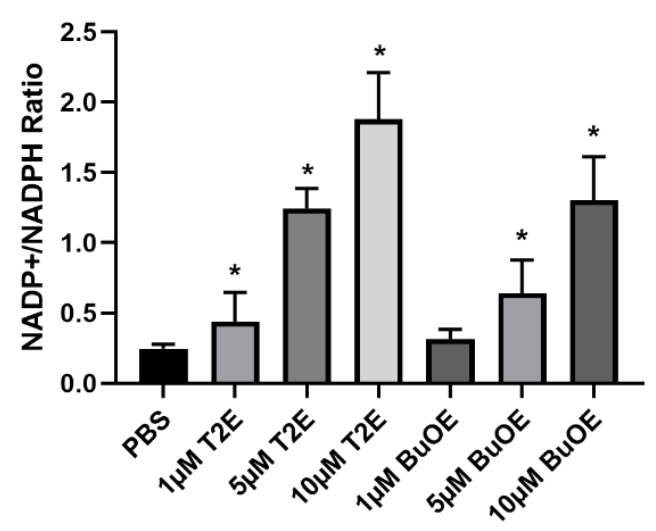


Figure 35. MnTE-2-PyP depleted NADPH pools in PC3 and LNCaP cells as measured by HPLC and mass spectrometry analysis. PC3 and LNCaP cells were seeded in media containing PBS or $30 \mu \mathrm{M}$ MnTE-2-PyP. After 24 hours, some groups of cells were exposed to 2 Gy radiation. After another 72 hours, cellular $N A D(P) H$ and $N A D(P)+$ levels were determine by the HPLC and mass spectrometry analysis. $(A) N A D(P)+/ N A D(P) H$ levels and ratios in PC3 cells. (B) NAD(P)+/NAD(P)H levels and ratios in LNCaP cells. All data represent mean \pm SD from at least four independent experiments. ${ }^{*} p<0.05$ compared to PBS treatment. 
A
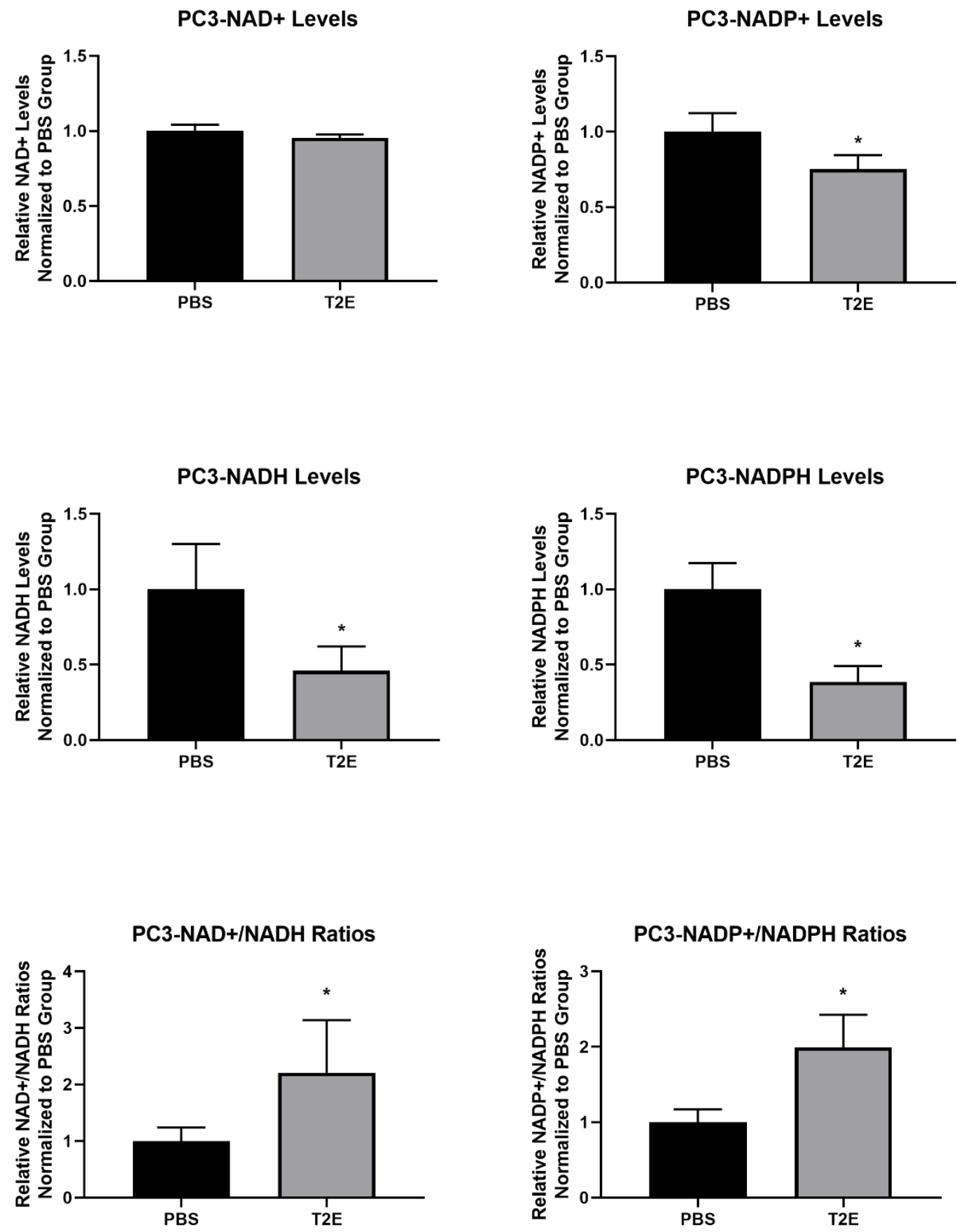
B

LNCaP-NAD+ Levels

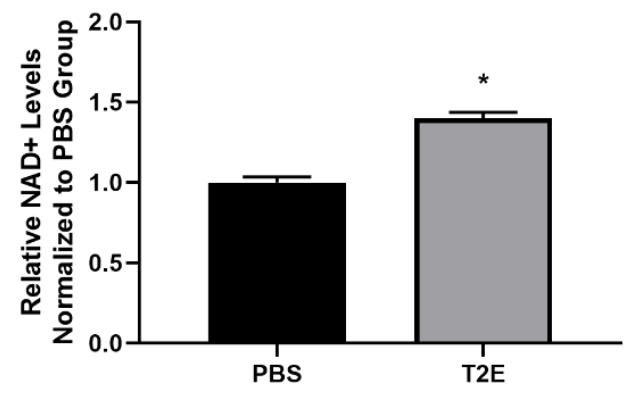

LNCaP-NADH Levels

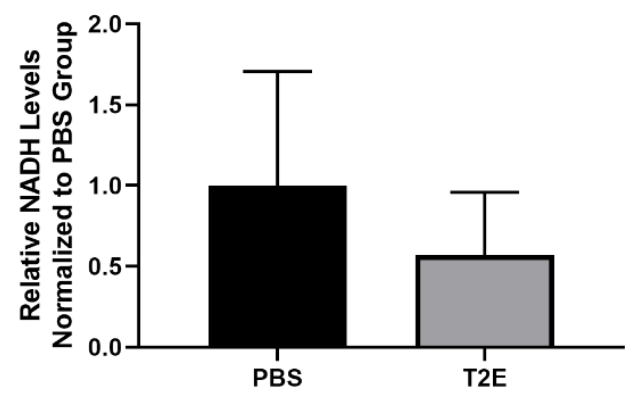

LNCaP-NAD+/NADH Ratios

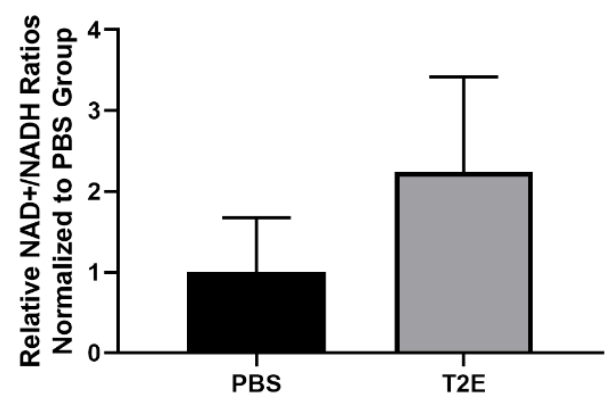

LNCaP-NADP+ Levels

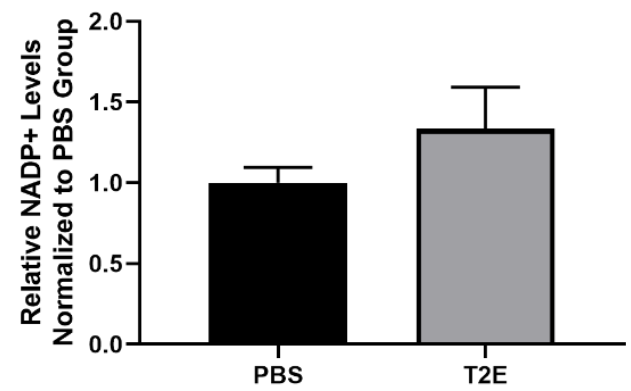

LNCaP-NADPH Levels

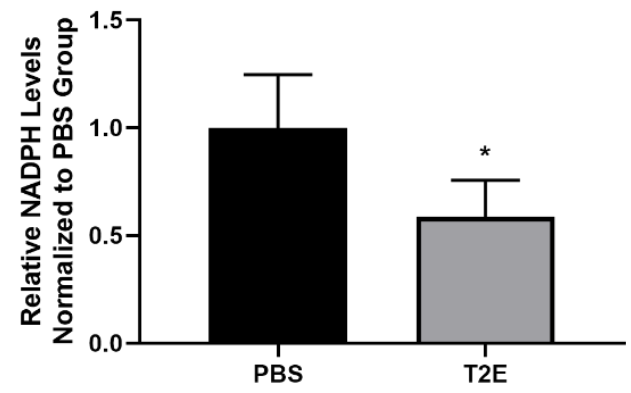

LNCaP-NADP+/NADPH Ratios

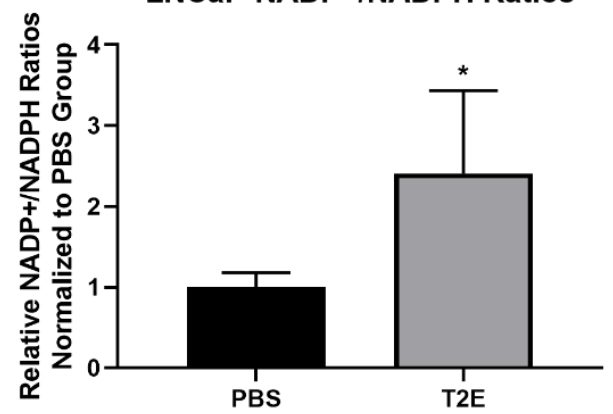


Figure 36. MnTE-2-PyP increased succinate levels in LNCaP cells but did not alter FAD/FADH ${ }_{2}$ levels in PC3 and LNCaP cells as measured by HPLC and mass spectrometry analysis. PC3 and LNCaP cells were seeded in media containing PBS or $30 \mu \mathrm{M}$ MnTE-2-PyP. After 24 hours, some groups of cells were exposed to 2 Gy radiation. After another 72 hours, cellular FAD/FADH 2 and succinate levels were determine by the HPLC and mass spectrometry analysis. (A) FAD/FADH 2 levels and ratios in PC3 cells. (B) FAD/FADH $H_{2}$ levels and ratios in LNCaP cells. (C) Succinate levels in PC3 and LNCaP cells. All data represent mean \pm SD from at least four independent experiments. ${ }^{*} p<0.05$ compared to PBS treatment. 
A
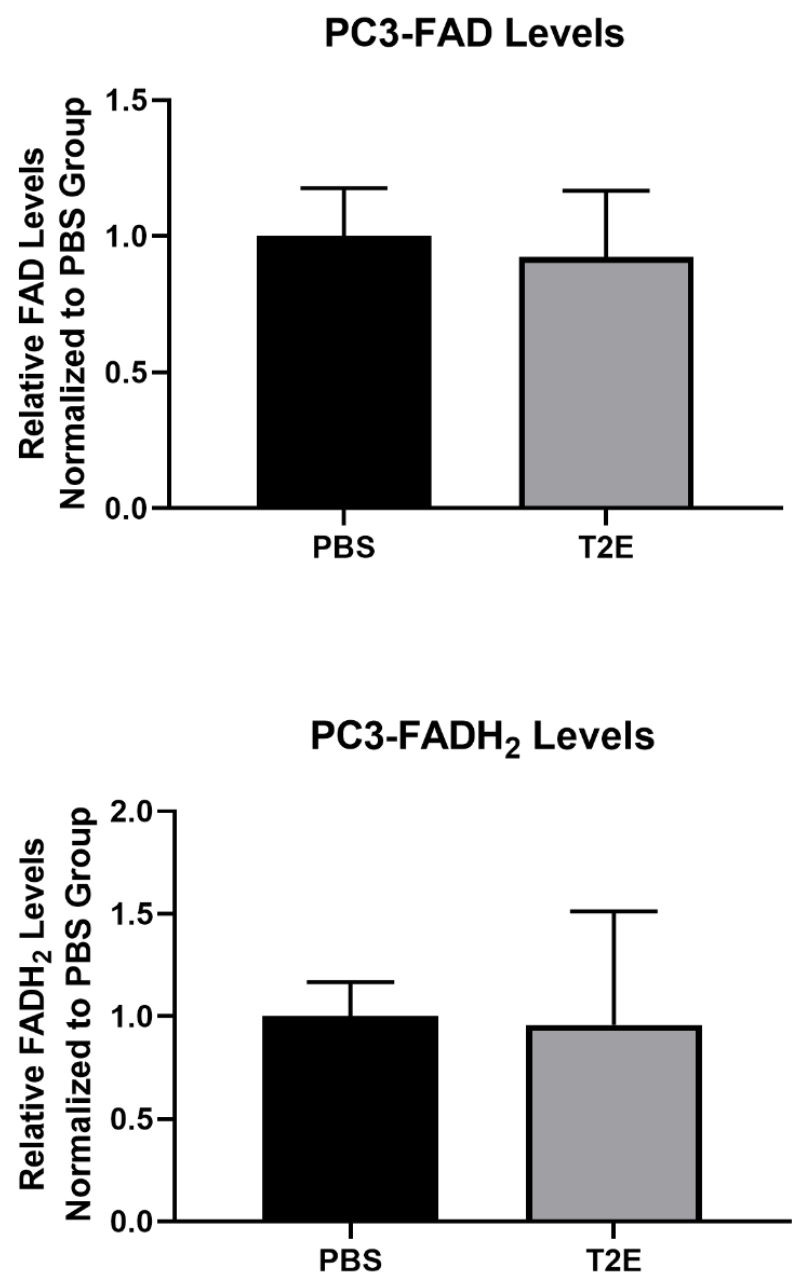

PC3-FAD/FADH ${ }_{2}$ Ratios

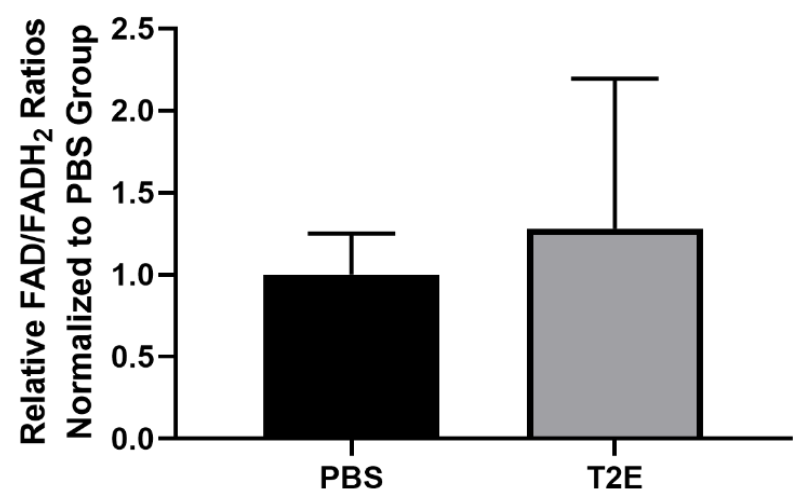


B

\section{LNCaP-FAD Levels}

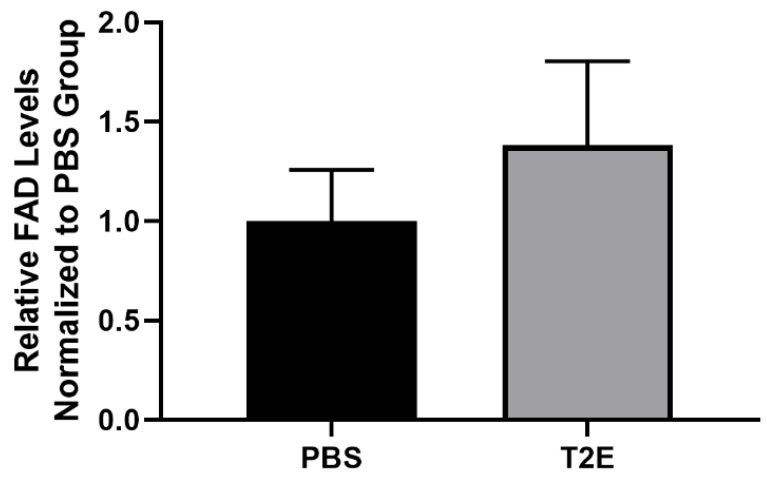

LNCaP-FADH ${ }_{2}$ Levels

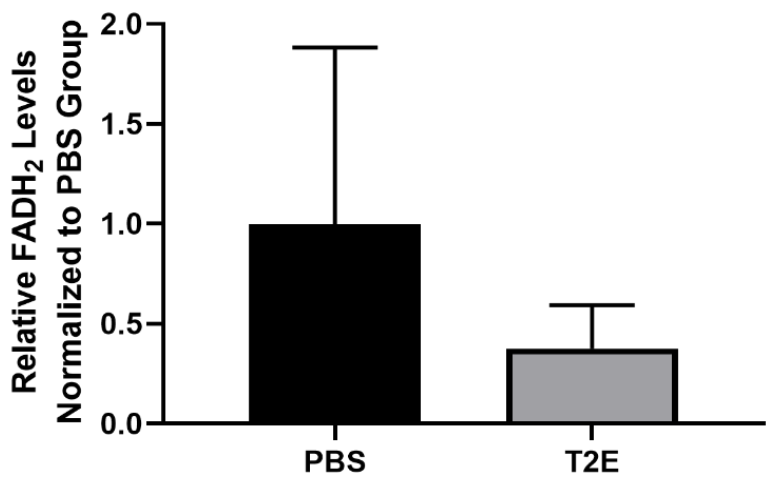

LNCaP-FAD/FADH ${ }_{2}$ Ratios

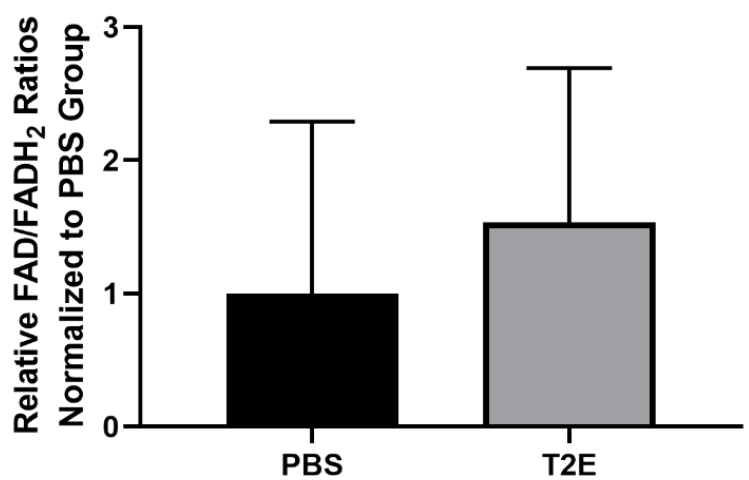


C

PC3-Succinate Levels

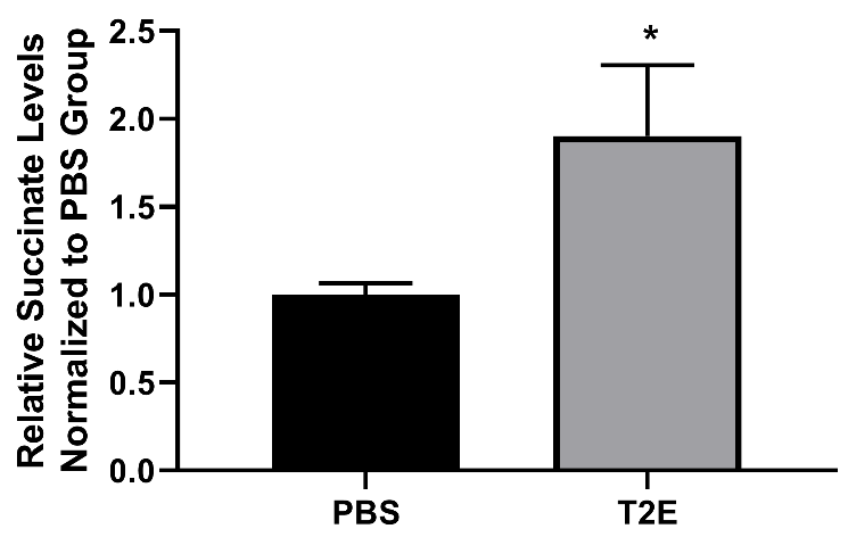

LNCaP-Succinate Levels

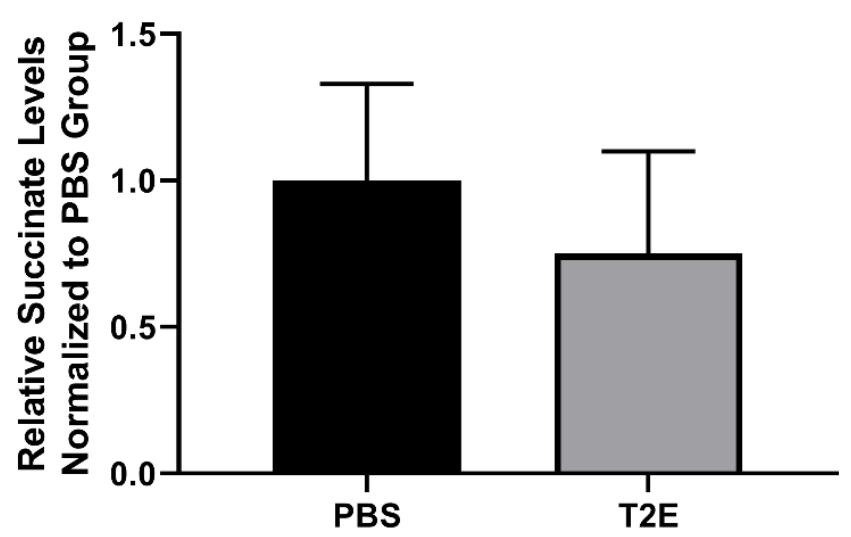




\subsection{Investigating potential factors/pathways that result in NADPH depletion}

The MnTE-2-PyP-induced alterations of NADP+/NADPH ratios can result from different reasons. As a central molecule involved in the redox defense system, the increase of ROS $\left(\mathrm{H}_{2} \mathrm{O}_{2}\right)$ levels may lead to NADPH level decreases. On the other hand, MnTE-2-PyP leads to cysteine modifications on proteins targets that are parts of the energy metabolism system and it is possible that enzymes generating or regulating NADPH are affected by MnTE-2-PyP, e.g. the glucose-6-phosphate dehydrogenase (G6PD) and 6-phosphogluconate dehydrogenase (6PGD) in the pentose phosphate pathway.

To determine whether the $\mathrm{H}_{2} \mathrm{O}_{2}$ level increase by MnTE-2-PyP is contributing to NADPH depletion, we transduced PC3 and LNCaP cells with catalase adenovirus, which scavenges intracellular $\mathrm{H}_{2} \mathrm{O}_{2}$ (Figure 37 ). Although catalase transduction significantly increased NADPH levels in PC3 cells, it did not rescue NADPH loss in the presence of MnTE-2-PyP in PC3 or LNCaP cells.

We also investigated whether NOX enzymes are responsible for the decreased NADPH levels because NOXs consume NADPH and produce $\mathrm{H}_{2} \mathrm{O}_{2}$ and superoxide, both of which could lead to decreased NADPH levels [172]. However, we found that blocking NOXs activity with the general NOX inhibitor, diphenyleneiodonium (DPI), did not alter the NADPH levels (Figure 38), which indicates that NOXs are not involved in the MnTE-2PyP-induced NADPH levels decrease.

The major cytosolic source of NADPH is the pentose phosphate pathway. The first and rate-limiting enzyme of pentose phosphate pathway is glucose-6-phosphate dehydrogenase $[111,129]$. Since $\mathrm{H}_{2} \mathrm{O}_{2}$ or $\mathrm{NOX}$ were not responsible for NADPH 
depletion, we further measured the G6PD activity in MnTE-2-PyP-treated prostate cancer cells. Interestingly, MnTE-2-PyP suppressed the G6PD activity in both PC3 and LNCaP cells by $40 \sim 50 \%$. In addition, radiation did not show inhibitory effects on G6PD (Figure 39). We next measured the 6PGD activity in MnTE-2-PyP-treated prostate cancer cells (Figure 40), and we observed similar results as compared to G6PD assay. MnTE-2-PyP significantly suppressed the production of NADPH by 6PGD in both PC3 ( $80 \%$ decrease) and LNCaP ( $50 \%$ decrease) cells. The above results indicate that the inhibition of pentose phosphate pathway could be one major reason for cellular NADPH depletion in prostate cancer cells. 
Figure 37. Catalase transduction did not rescue NADPH ratios in MnTE-2-PyP. treated prostate cancer cells. PC3 and LNCaP cells were transduced with 50 or $10 \mathrm{MOI}$ catalase adenovirus, respectively. After 72 hours, cells were treated with PBS or $30 \mu \mathrm{M}$ MnTE-2-PyP for 6 hours, and the cellular NADPH and NADP+ levels were determine by the NADP+/NADPH-Glo assay. (A) NADP+/NADPH ratios, NADP+ and NADPH levels in PC3 cells with different treatments. (B) NADP+/NADPH ratios, NADP+ and NADPH levels in LNCaP cells with different treatments. All data represent mean \pm SD from at least three independent experiments. ${ }^{*} p<0.05$ compared to PBS treatment. 
A
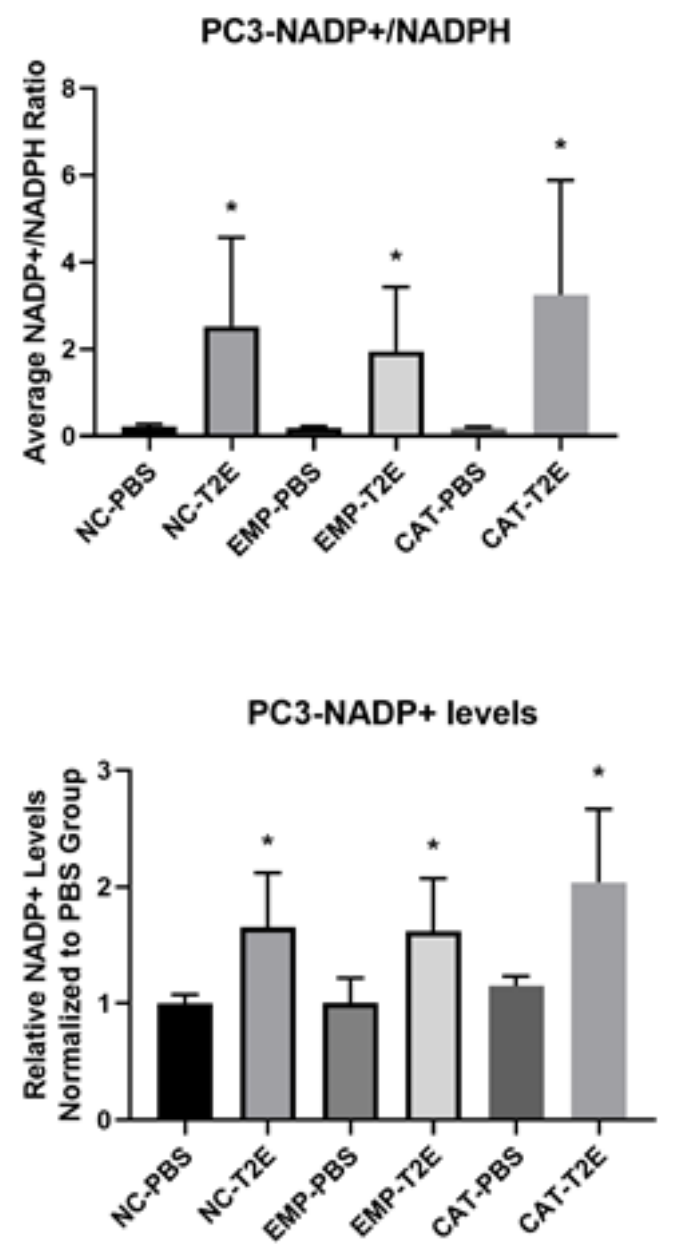

PC3-NADPH levels

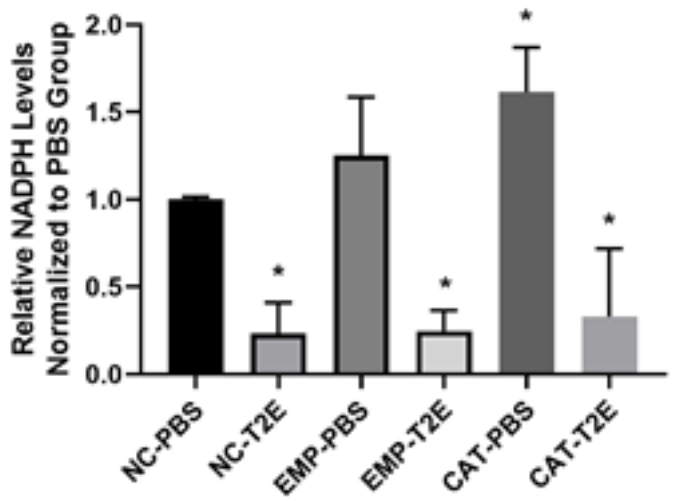


B

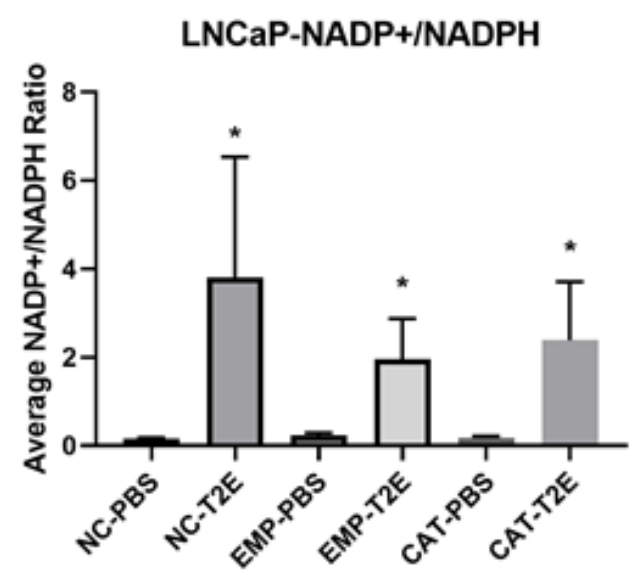

LNCaP-NADP+ levels

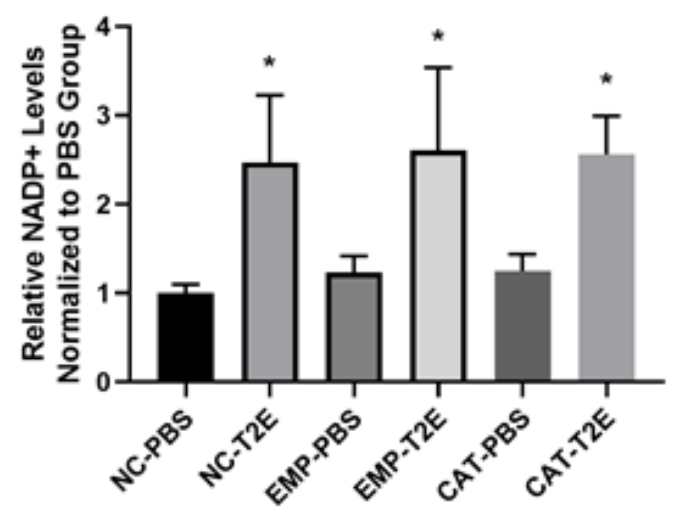

LNCaP-NADPH

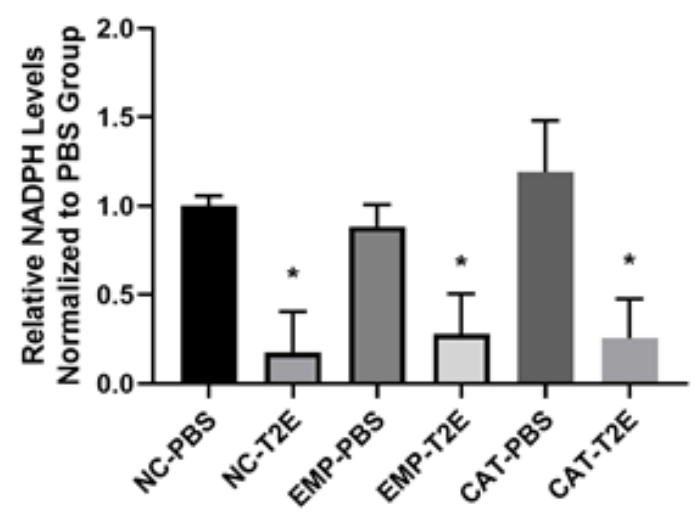


Figure 38. NOX enzymes were not involved in MnTE-2-PyP-induced NADPH levels decrease. PC3 and LNCaP cells were treated with PBS or $30 \mu \mathrm{M}$ MnTE-2-PyP with or without $2 \mu \mathrm{M}$ DPI for 6 hours, and the cellular NADPH and NADP+ levels were determine by the NADP+/NADPH-Glo assay. (A) NADP+/NADPH ratios, NADP+ and NADPH levels in PC3 cells with different treatments. (B) NADP+/NADPH ratios, NADP+ and NADPH levels in LNCaP cells with different treatments. All data represent mean \pm SD from at least three independent experiments. ${ }^{*} p<0.05$ compared to PBS treatment. 
A
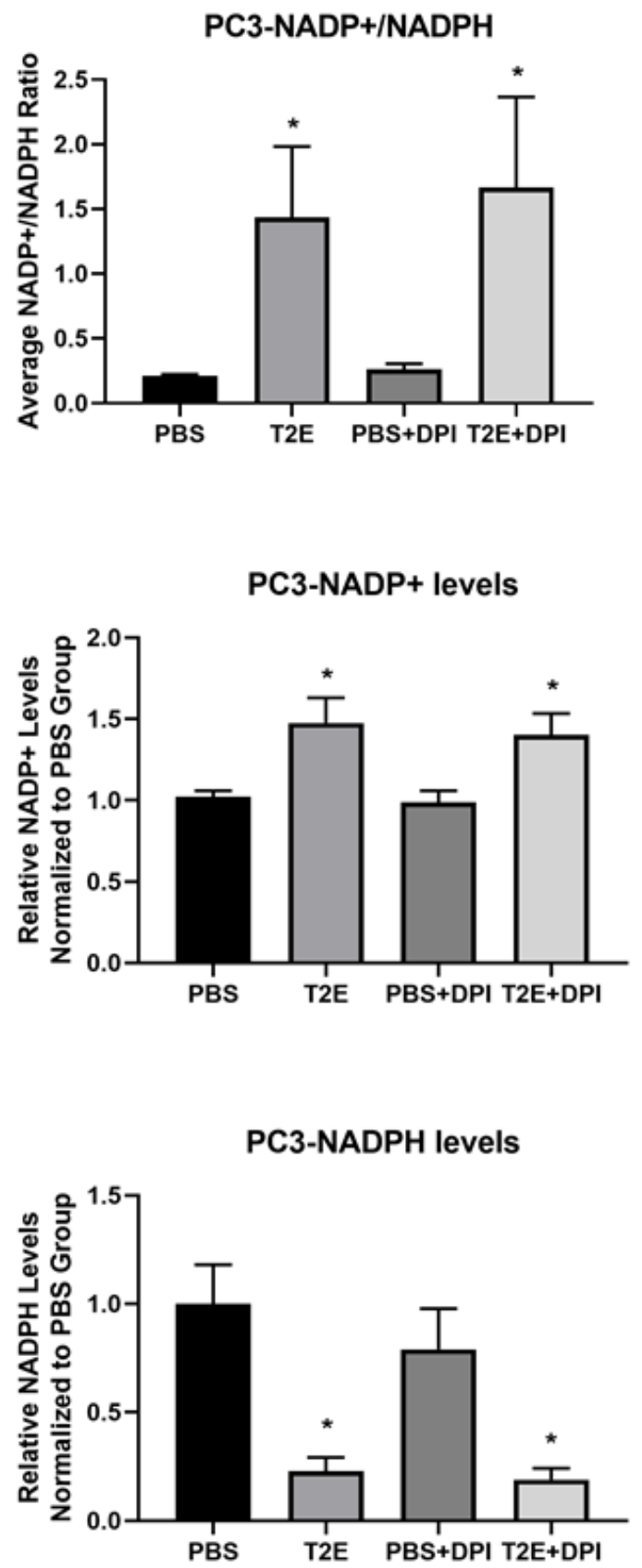
B

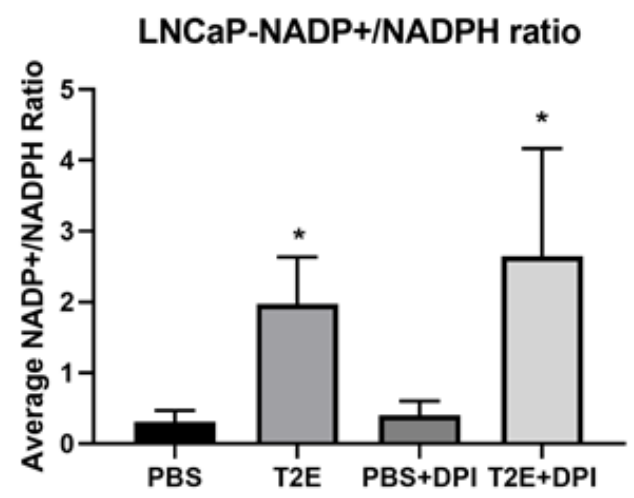

LNCaP-NADP+ levels

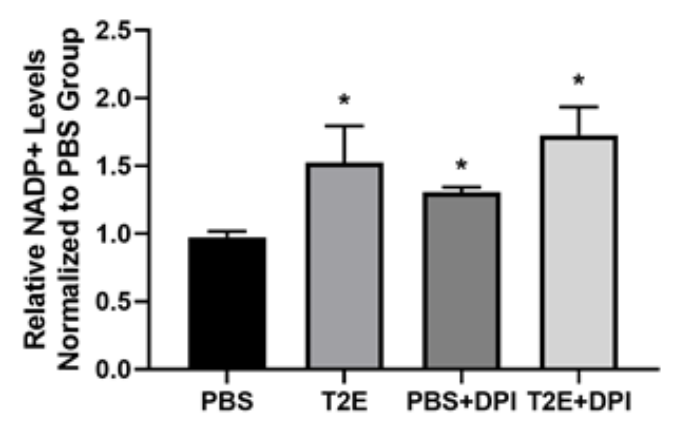

LNCaP-NADPH levels

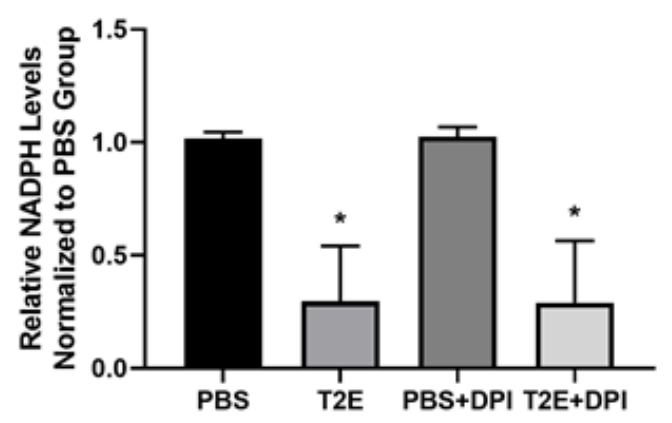


Figure 39. MnTE-2-PyP resulted in G6PD inhibition in prostate cancer cells. PC3 and LNCaP cells were seeded in media containing PBS or $30 \mu \mathrm{M}$ MnTE-2-PyP. After 24 hours, some groups of cells were exposed to 2 Gy radiation. After another 72 hours, G6PD activity was measured by the G6PD activity assay. All data represent mean \pm SD from at least three independent experiments. ${ }^{*} p<0.05$ compared to PBS treatment. 
PC3

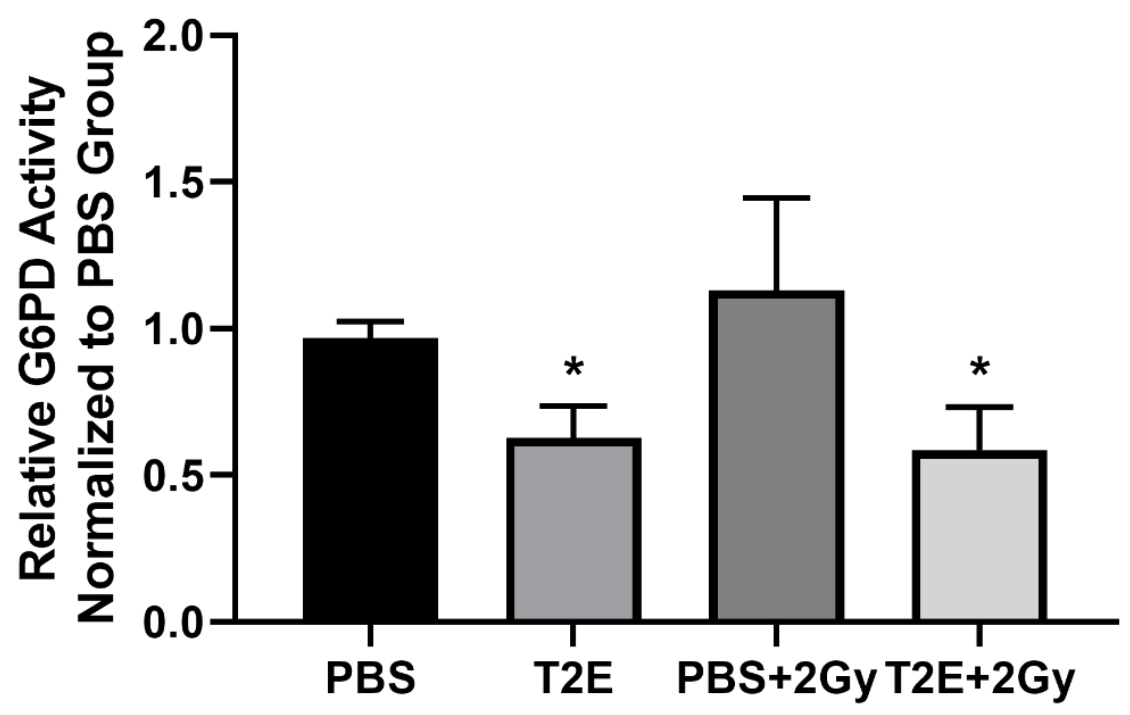

LNCaP

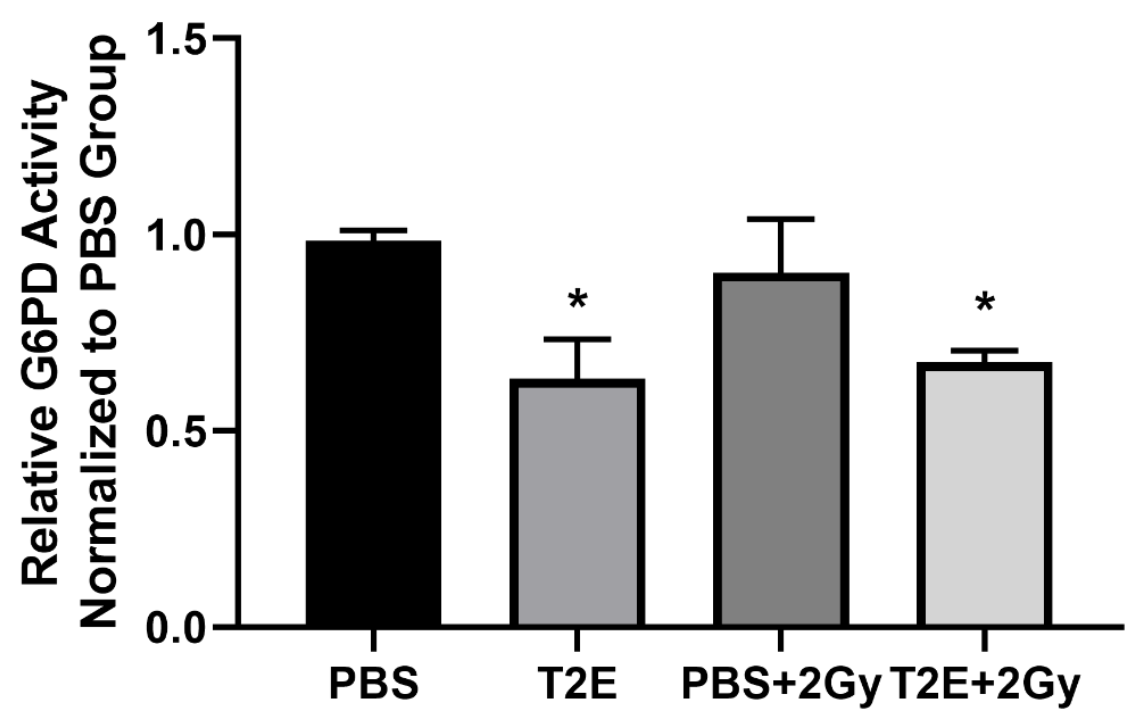


Figure 40. MnTE-2-PyP resulted in 6PGD inhibition in prostate cancer cells. PC3 and LNCaP cells were seeded in media containing PBS or $30 \mu \mathrm{M}$ MnTE-2-PyP. After 24 hours, some groups of cells were exposed to 2 Gy radiation. After another 72 hours, 6PGD activity was measured by the 6PGD activity assay. All data represent mean \pm SD from at least three independent experiments. ${ }^{*} p<0.05$ compared to PBS treatment of PC3 cells; $\# p<0.05$ compared to PBS treatment of LNCaP cells. 


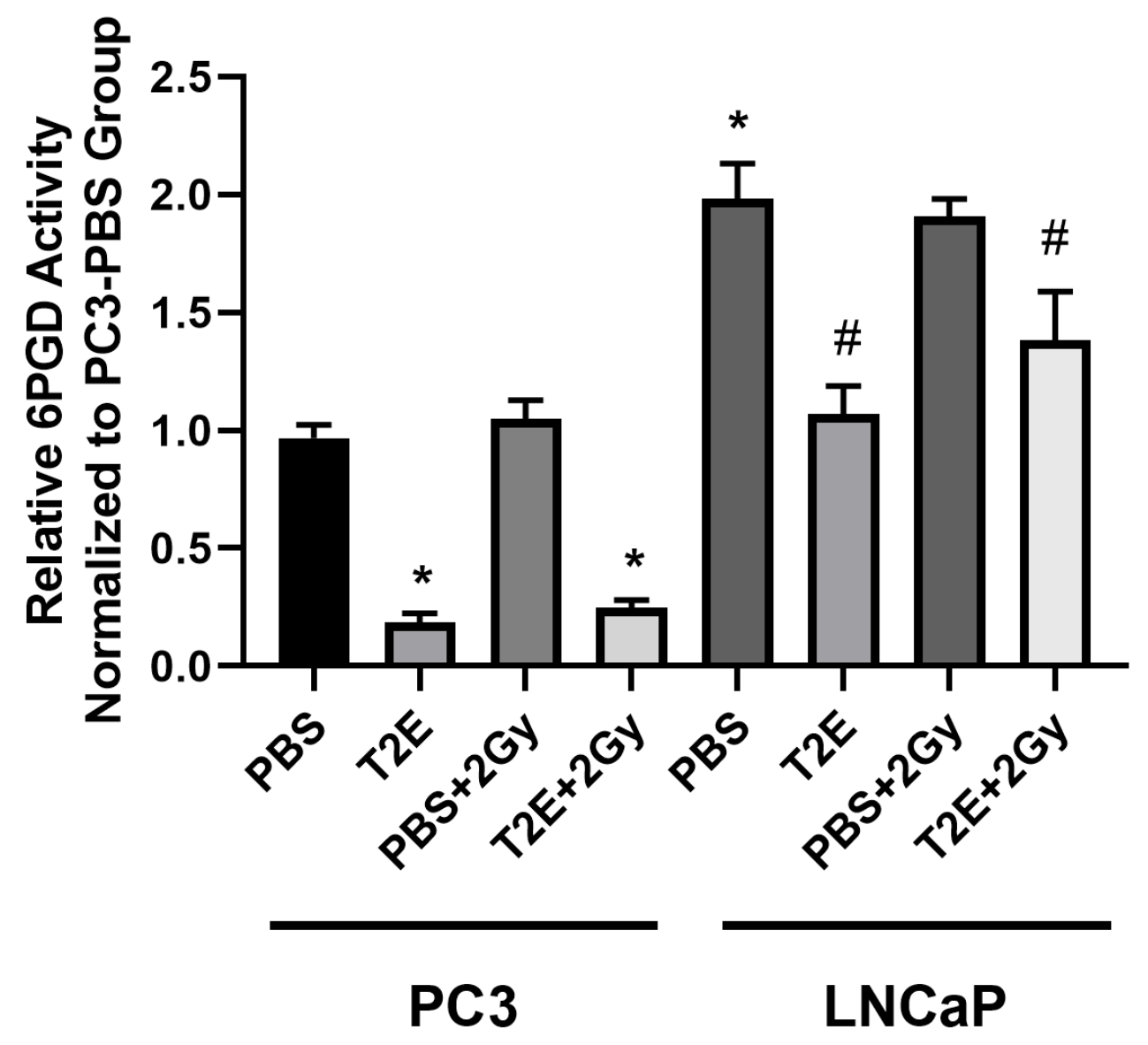




\subsection{The effects of MnTE-2-PyP on mitochondrial $\mathrm{H}_{2} \mathrm{O}_{2}$ and $\mathrm{ROS}$ levels in prostate cancer cells}

We have shown that MnTE-2-PyP significantly altered NAD+/NADH and $\mathrm{NADP}+/ \mathrm{NADPH}$ ratios in prostate cancer cells, which can affect both energy metabolism and redox balance. To evaluate the redox states in mitochondria, we measured mitochondrial $\mathrm{H}_{2} \mathrm{O}_{2}$ and superoxide levels in PC3 and LNCaP cells. Unexpectedly, we found that radiation, rather than MnTE-2-PyP, was able to increase mitochondrial $\mathrm{H}_{2} \mathrm{O}_{2}$ levels in both prostate cancer cell lines (Figure 41A). Moreover, PC3 cells have very low basal level mitochondrial $\mathrm{H}_{2} \mathrm{O}_{2}$, indicating mitochondrial $\mathrm{H}_{2} \mathrm{O}_{2}$ levels change may not play an important role in MnTE-2-PyP-induced cell growth inhibition. In addition, overall mitochondrial ROS levels were suppressed by MnTE-2-PyP in PC3 cells, but enhanced by radiation in LNCaP cells (Figure 41B). Based on the inconsistent trends of mitochondrial $\mathrm{H}_{2} \mathrm{O}_{2}$ and ROS levels in prostate cancer cells, we believed that these changes could be due to a response of PC3 and LNCaP cells to MnTE-2-PyP treatment, instead of being major contributors to MnTE-2-PyP-induced cell growth inhibition. 
Figure 41. MnTE-2-PyP and radiation affect mitochondrial $\mathrm{H}_{2} \mathrm{O}_{2}$ and overall ROS levels in prostate cancer cells. PC3 and LNCaP cells were seeded in media containing PBS or $30 \mu \mathrm{M}$ MnTE-2-PyP. After 24 hours, some groups of cells were exposed to 2 Gy radiation. After another 72 hours, (A) mitochondrial $\mathrm{H}_{2} \mathrm{O}_{2}$ and (B) mitochondrial overall ROS levels were determined by MitoPY-1 and MitoSOX by FACS, respectively. All data represent mean \pm SD from at least three independent experiments. ${ }^{*} p<0.05$ compared to PBS treatment; \#p<0.05 compared to PBS + 2Gy treatment. 
A

Mitochondrial $\mathrm{H}_{2} \mathrm{O}_{2}$
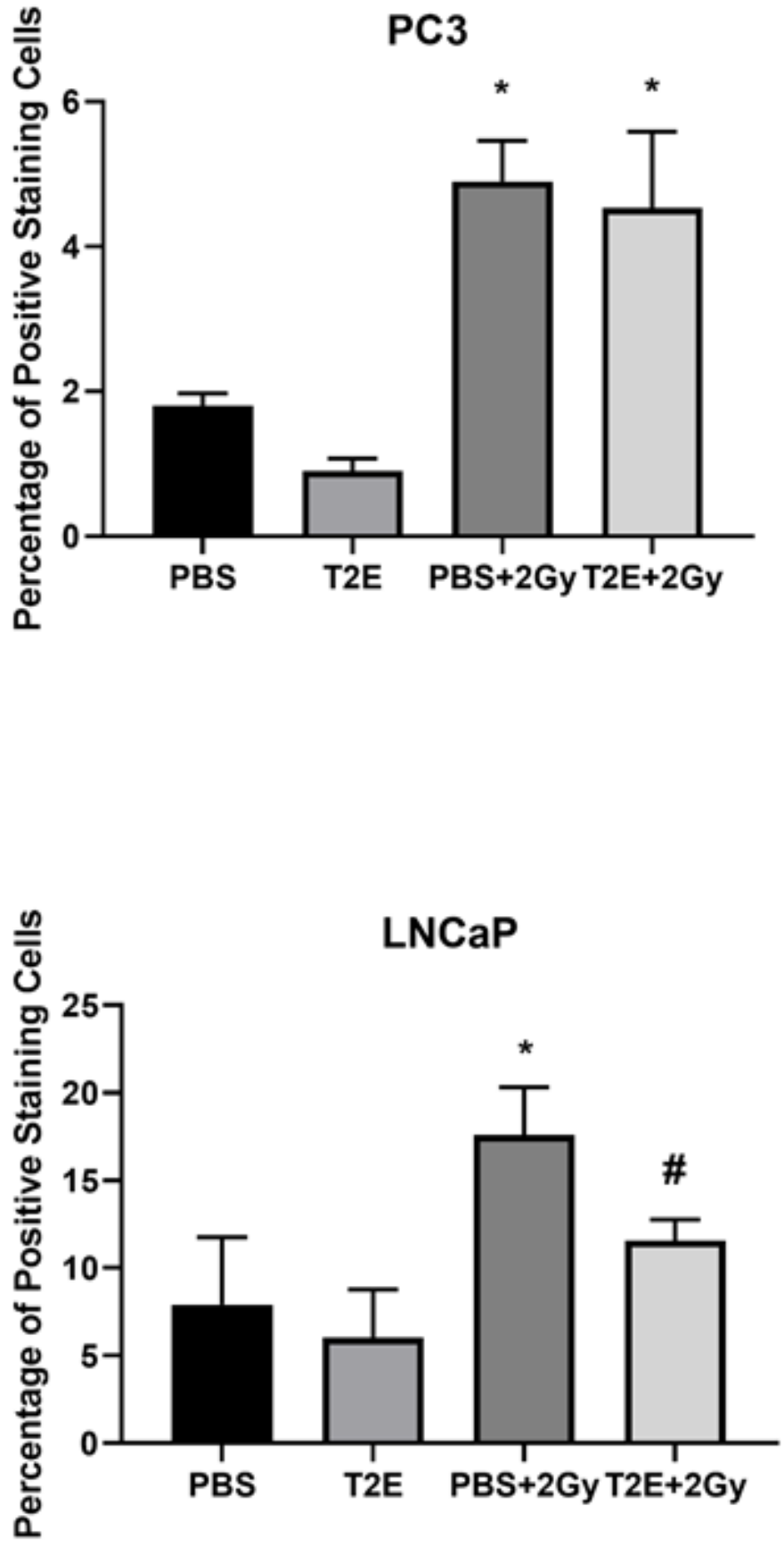
B

Mitochondrial ROS
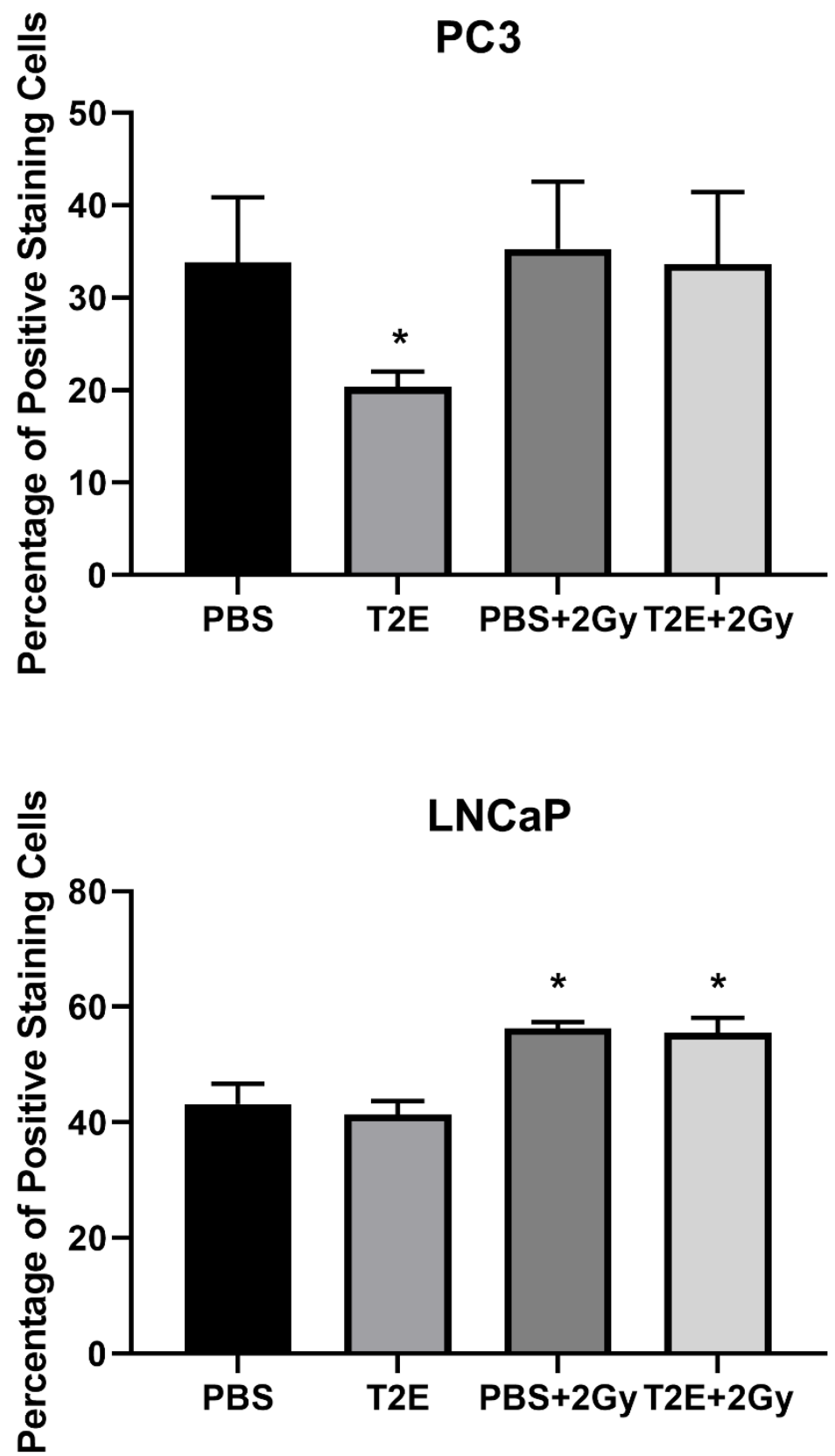


\subsection{The effects of MnTE-2-PyP on GSH and GSSG levels in prostate cancer cells}

GSH and GSSG are a pair of small molecules that plays a crucial role in the cellular antioxidant defense system (Figure 2). The NADPH depletion by MnTE-2-PyP treatment could potentially affect cellular GSH/GSSG ratios and disturb redox balance. Therefore, we measured GSH and GSSG levels in both PC3 and LNCaP cells with MnTE-2-PyP and radiation treatment. We found that MnTE-2-PyP significantly reduced GSH/GSSG ratios in both PC3 and LNCaP cells (Figure 42A); however, the cause of these changes were different in PC3 and LNCaP cells. In PC3 cells, there was a decrease of GSH levels in MnTE-2-PyP-treated cells, but GSSG levels were not affected by either MnTE-2-PyP or radiation treatment (Figure 42B). In LNCaP cells, MnTE-2-PyP significantly enhanced GSSG levels, while GSH levels were not affected by either MnTE-2-PyP or radiation treatment (Figure 42C).

We also confirmed the GSH/GSSG levels data by the HPLC and mass spectrometry analysis (Figure 43). Consistently, GSH/GSSG ratios were decreased in both cell lines with MnTE-2-PyP treatment. In addition, the GSSG and total GSH+GSSG levels were also enhanced by the MnTE-2-PyP treatment, which indicates the de novo synthesis of GSH might be increased. Based on both glutathione assay kit and mass spectrometry analysis results, we found that MnTE-2-PyP treatment significantly decreased GSH/GSSG ratios in prostate cancer cells. 
Figure 42. MnTE-2-PyP and radiation affect GSH and GSSG levels in prostate cancer cells as measured by the glutathione assay kit. PC3 and LNCaP cells were seeded in media containing PBS or $30 \mu \mathrm{M}$ MnTE-2-PyP. After 24 hours, some groups of cells were exposed to 2 Gy radiation. After another 72 hours, cellular NADPH and NADP+ levels were determine by NADP+/NADPH-Glo assay. (A) GSH/GSSG ratios in PC3 and LNCaP cells with different treatments. (B) GSH and GSSG levels in PC3 cells with different treatments. (C) GSH and GSSG levels in LNCaP cells with different treatments. All data represent mean \pm SD from at least three independent experiments. ${ }^{*} p<0.05$ compared to PBS treatment. 
A

PC3-GSH/GSSG ratio

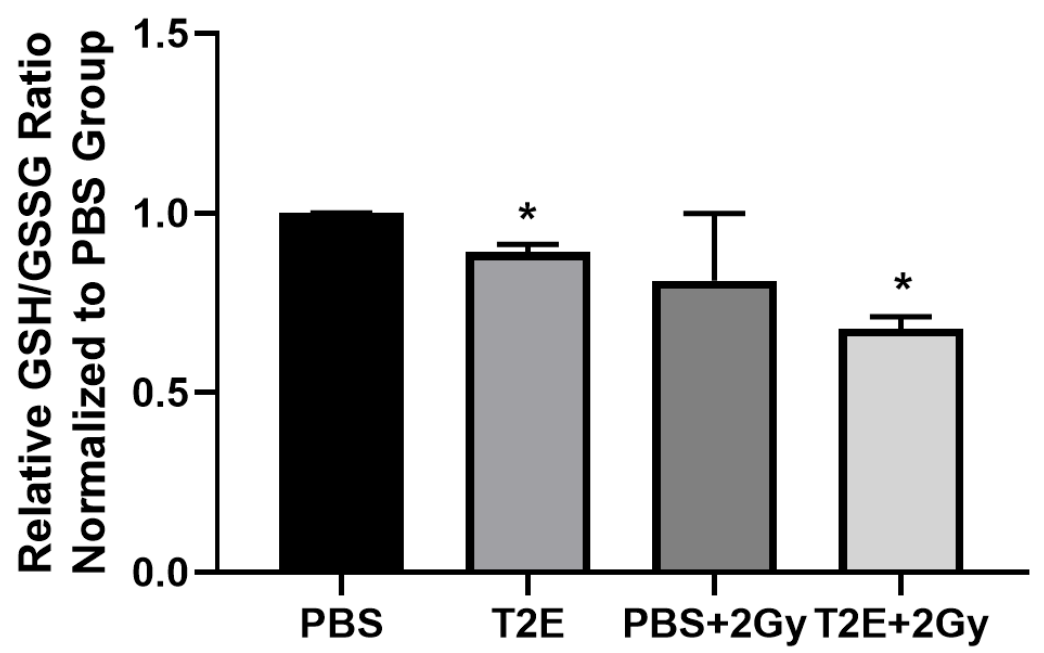

LNCaP-GSH/GSSG ratio

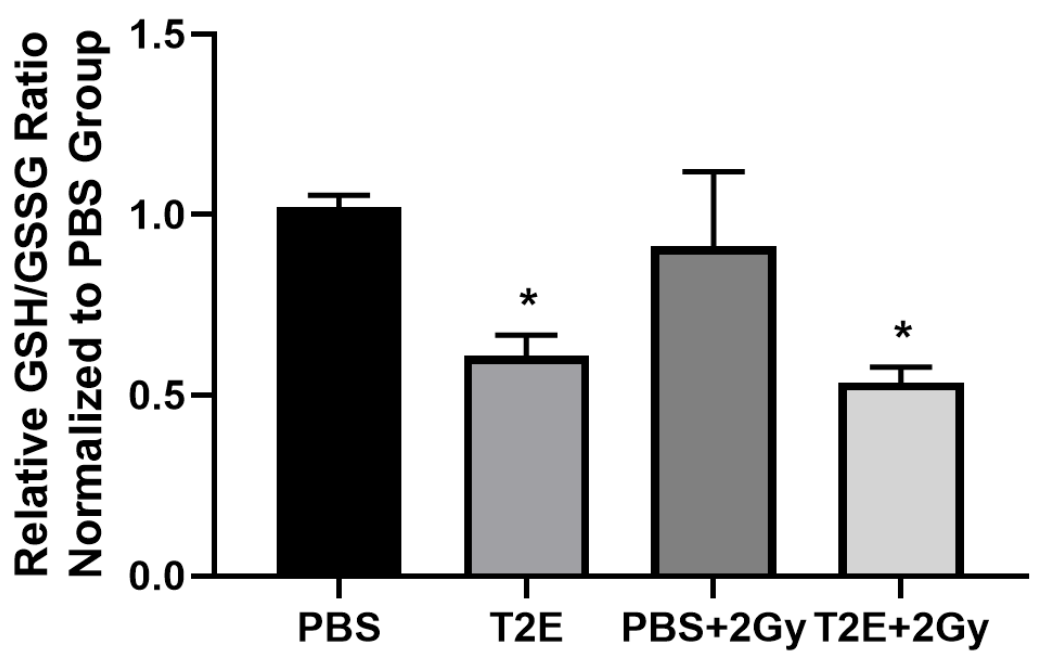


B

PC3-GSH

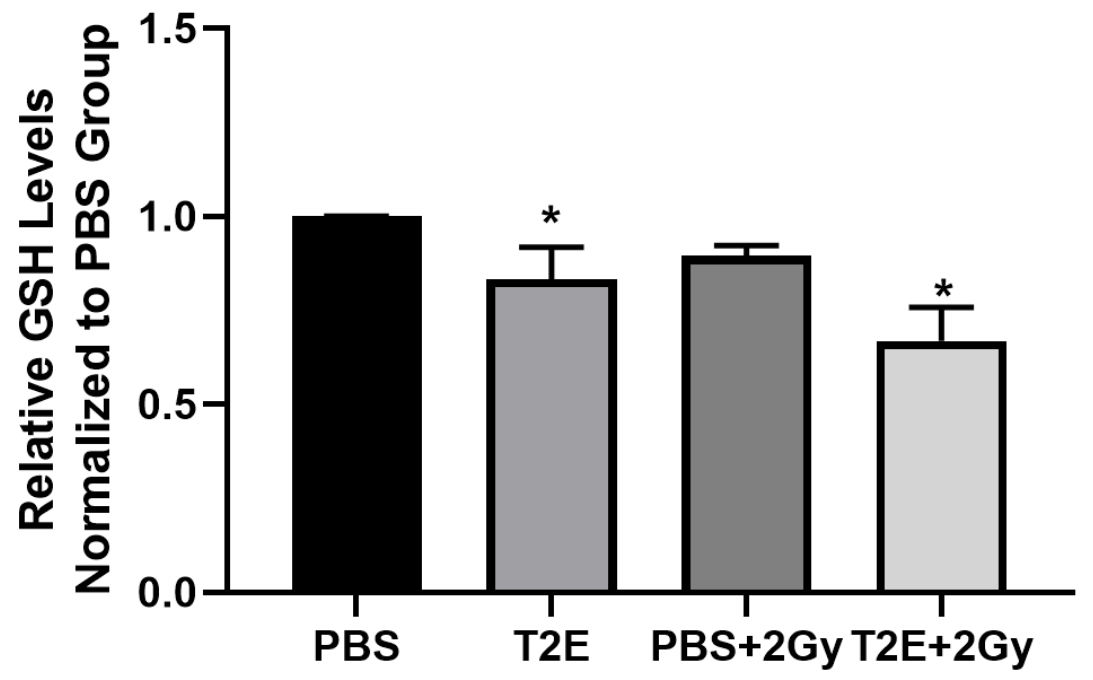

PC3-GSSG

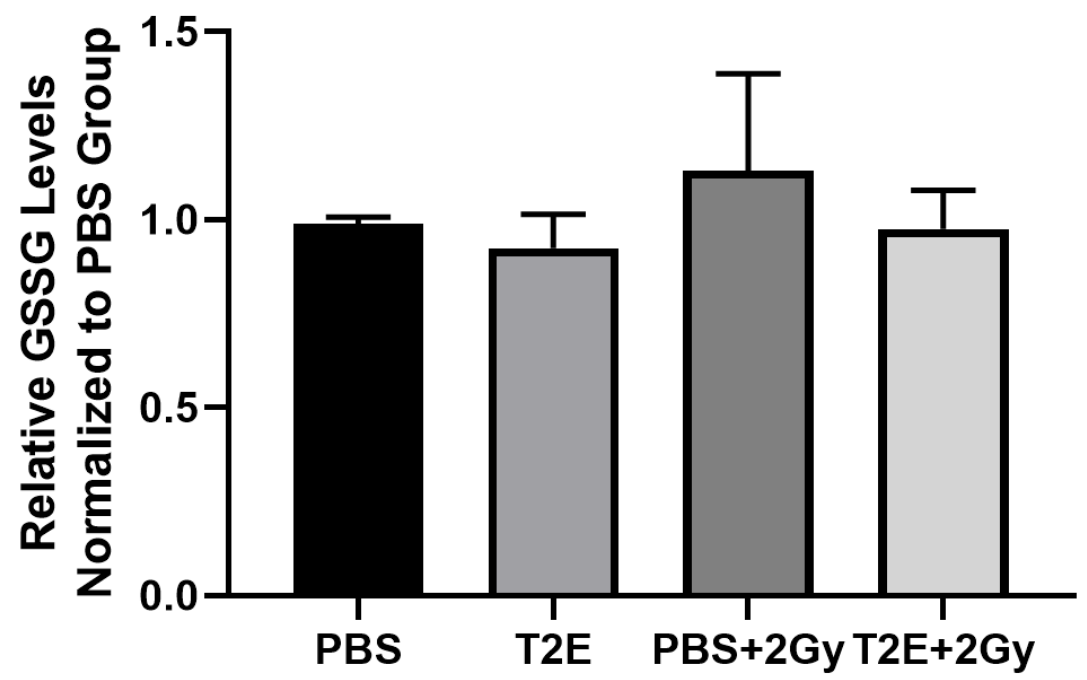


C

LNCaP-GSH

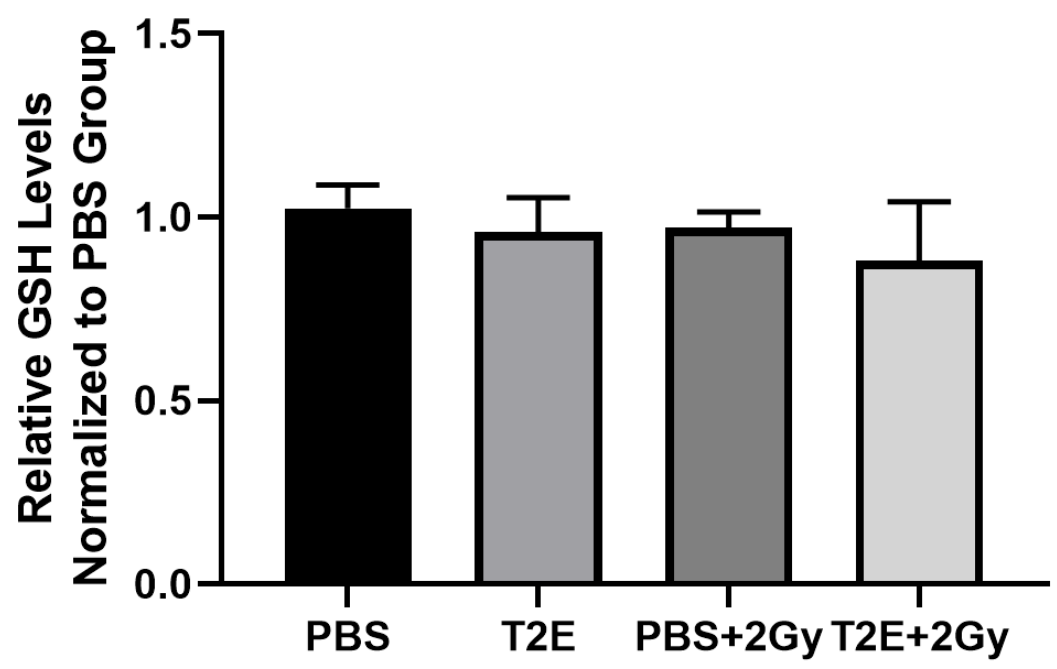

LNCaP-GSSG

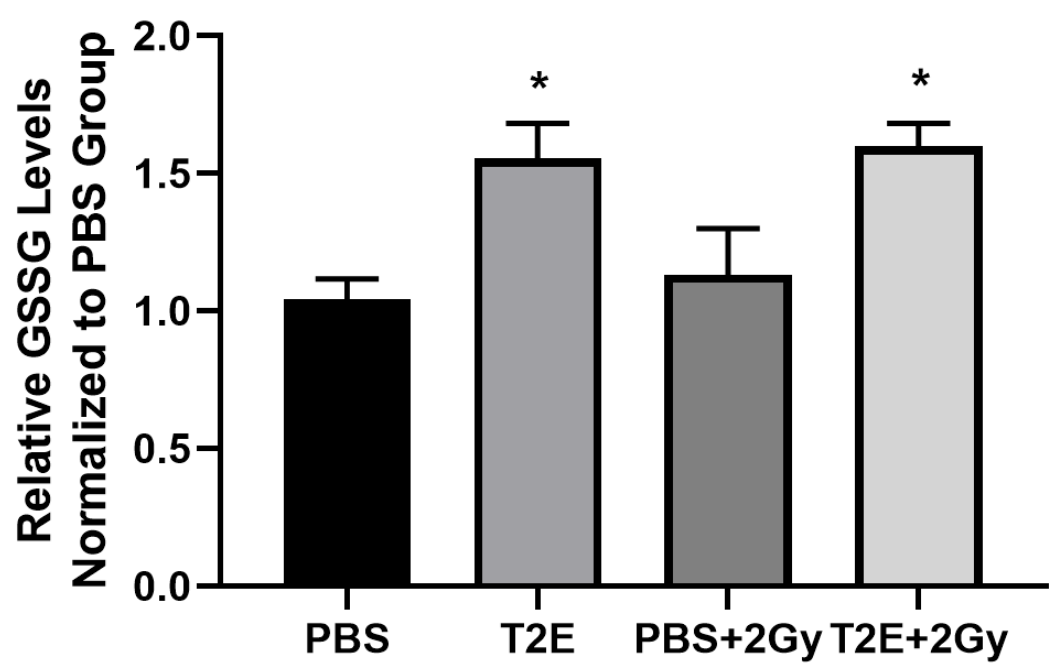


Figure 43. MnTE-2-PyP and radiation affect GSH and GSSG levels in prostate cancer cells identified by HPLC and mass spectrometry analysis. PC3 and LNCaP cells were seeded in media containing PBS or $30 \mu \mathrm{M}$ MnTE-2-PyP. After 24 hours, some groups of cells were exposed to 2 Gy radiation. After another 72 hours, cellular NADPH and NADP+ levels were determine by HPLC and mass spectrometry analysis. (A) GSH/GSSG ratios in PC3 and LNCaP cells with different treatments. (B) GSH and GSSG levels in PC3 cells with different treatments. (C) GSH and GSSG levels in LNCaP cells with different treatments. All data represent mean \pm SD from at least three independent experiments. ${ }^{*} p<0.05$ compared to PBS treatment. 
A

\section{PC3-GSH/GSSG Ratios}

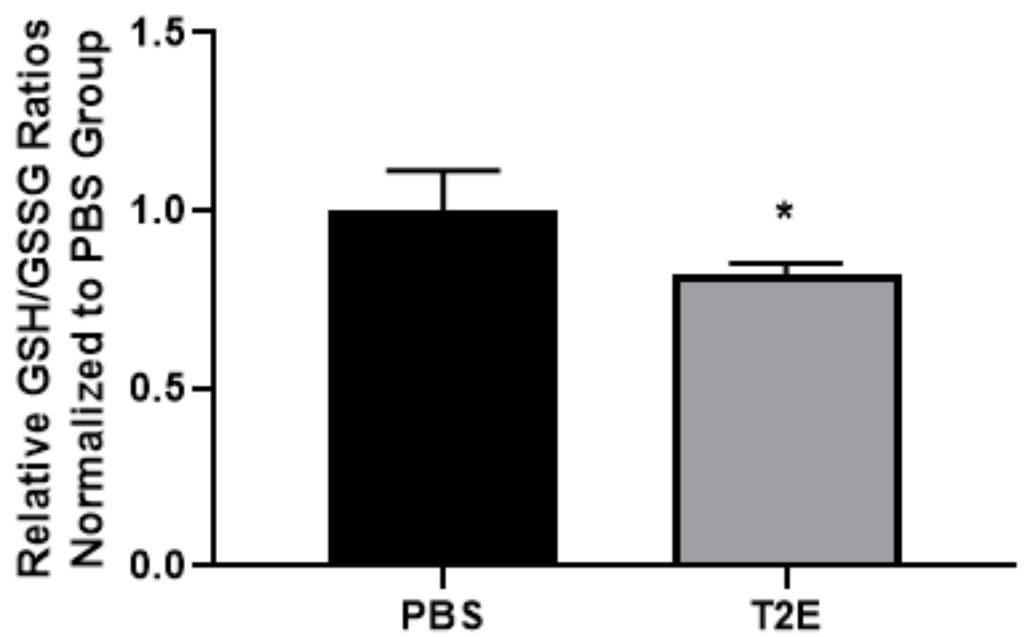

LNCaP-GSH/GSSG Ratios

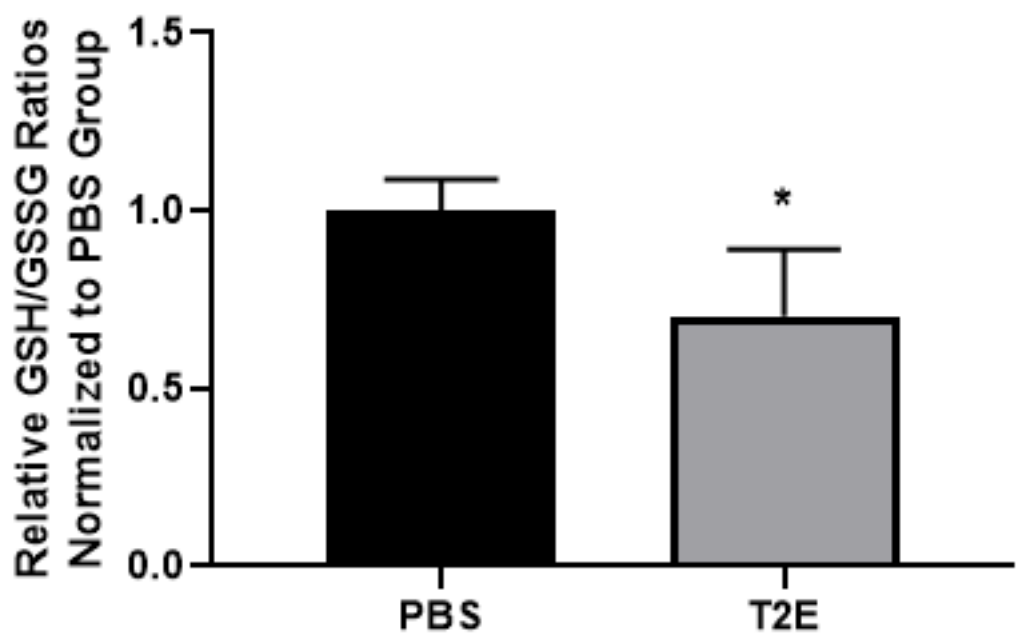


B

PC3-GSH Levels

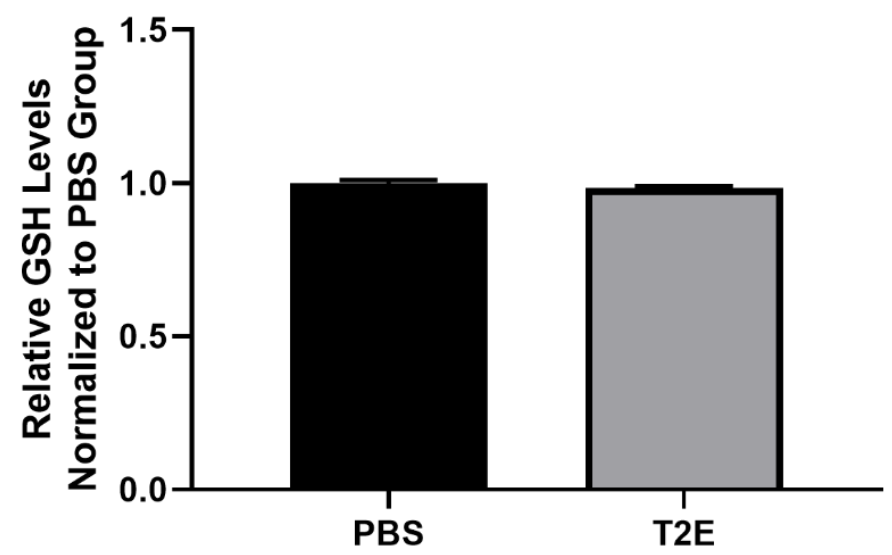

PC3-GSSG Levels

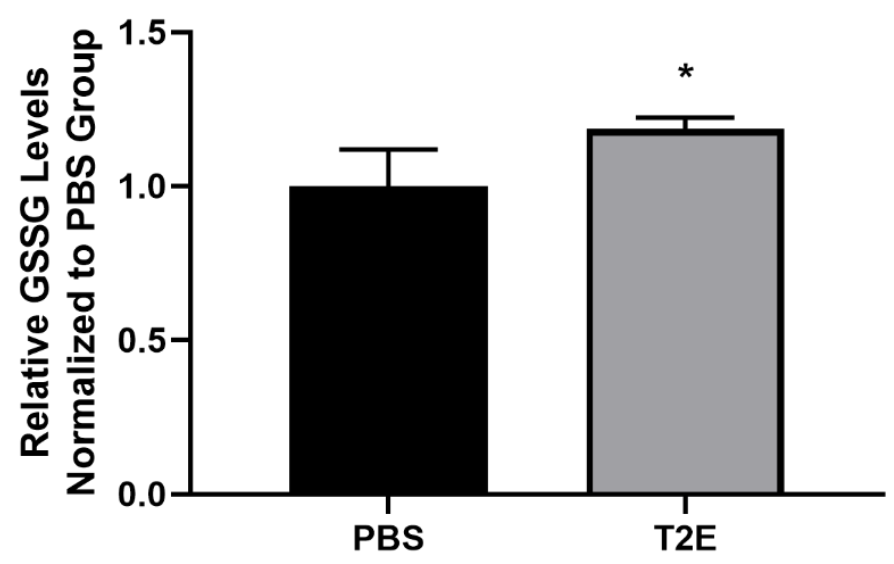


C

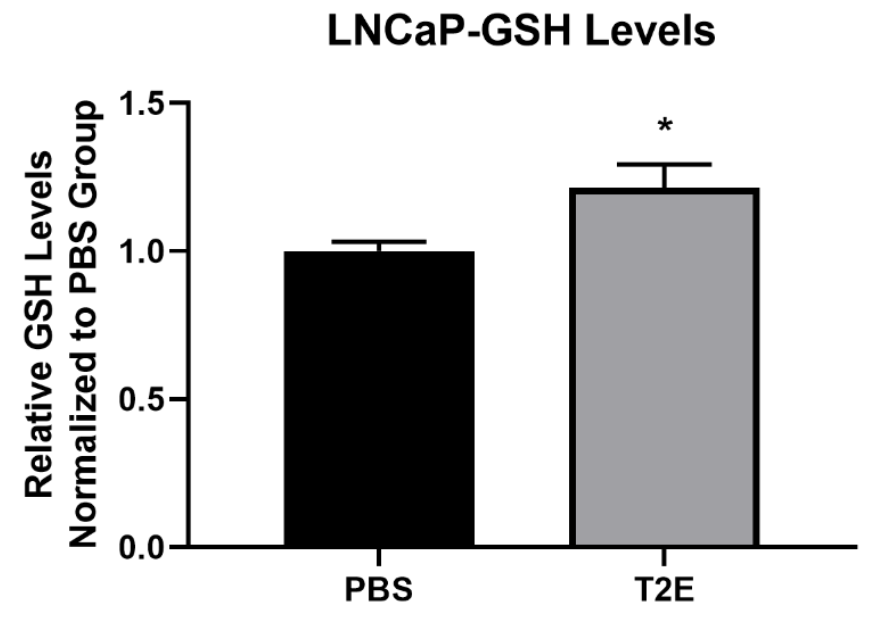

LNCaP-GSSG Levels

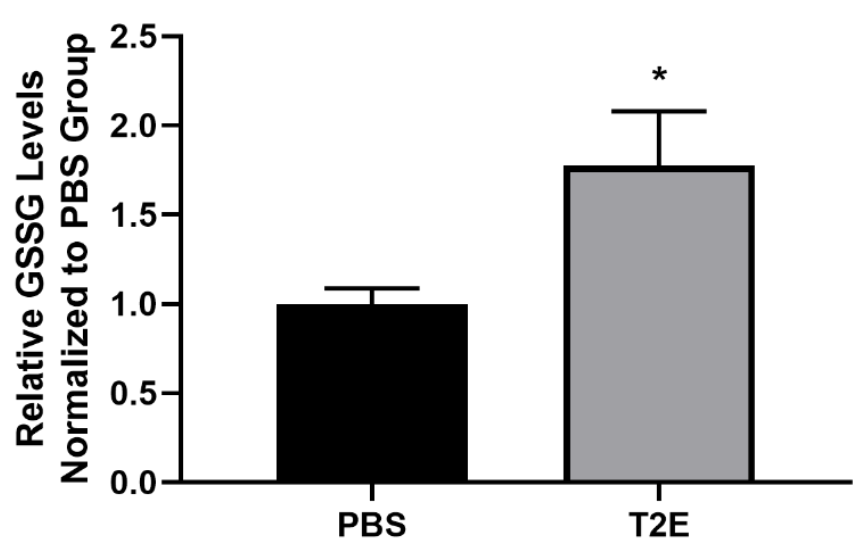




\subsection{MnTE-2-PyP inhibits GR activity by depleting NADPH pool}

We have observed a significant decrease of GSH/GSSG ratios in MnTE-2-PyPtreated PC3 and LNCaP cells, which may result from the loss of GR activity. To determine whether GR activity was altered in PC3 and LNCaP cells due to MnTE-2-PyP treatment, we first measured the GR activity by supplementing with sufficient NADPH. We found that LNCaP cells has much higher GR activity per cell than PC3 cells, and MnTE-2-PyP did not significantly altered GR activity in either cells lines (Figure 44A). To further investigate whether cellular NADPH levels contribute to the GSH/GSSG ratio change, we removed the NADPH addition step and only used cellular NADPH for GSSG reduction. We were unable to measure the GR activity in PC3 cells due to the extremely low basal activity without additional NADPH supplement. In LNCaP cells, we found that PBS condition showed significantly higher GR activity than other conditions, including the cells treated with MnTE-2-PyP alone or combined with radiation (Figure 44B). Therefore, the GSH/GSSG ratios alterations may be due to the decrease of NADPH pool in MnTE-2PyP-treated prostate cancer cells, instead of a direct decrease of GR enzyme activity. In addition, the huge difference of basal GR activity between PC3 and LNCaP cells may partially explain the huge difference of basal $\mathrm{H}_{2} \mathrm{O}_{2}$ levels as measured by ROS-Glo kit assays. 
Figure 44. MnTE-2-PyP may decrease GSH/GSSG ratios in prostate cancer cells by reducing cellular NADPH levels. PC3 and LNCaP cells were seeded in media containing PBS or $30 \mu \mathrm{M}$ MnTE-2-PyP. After 24 hours, some groups of cells were exposed to 2 Gy radiation. After another 72 hours, cellular GR activity was determined by the glutathione reductase activity assay. (A) Additional NADPH was provided for GR acitivity measurement in PC3 and LNCaP cells, MnTE-2-PyP did not significantly reduced NADPH levels in this experiment. (B) No NADPH was supplemented in this experiment, only cellular NADPH of LNCaP cells was available for GSSG reduction by GR. All data represent mean $\pm S D$ from at least three independent experiments. ${ }^{*} p<0.05$ compared to PBS treatment. 
A

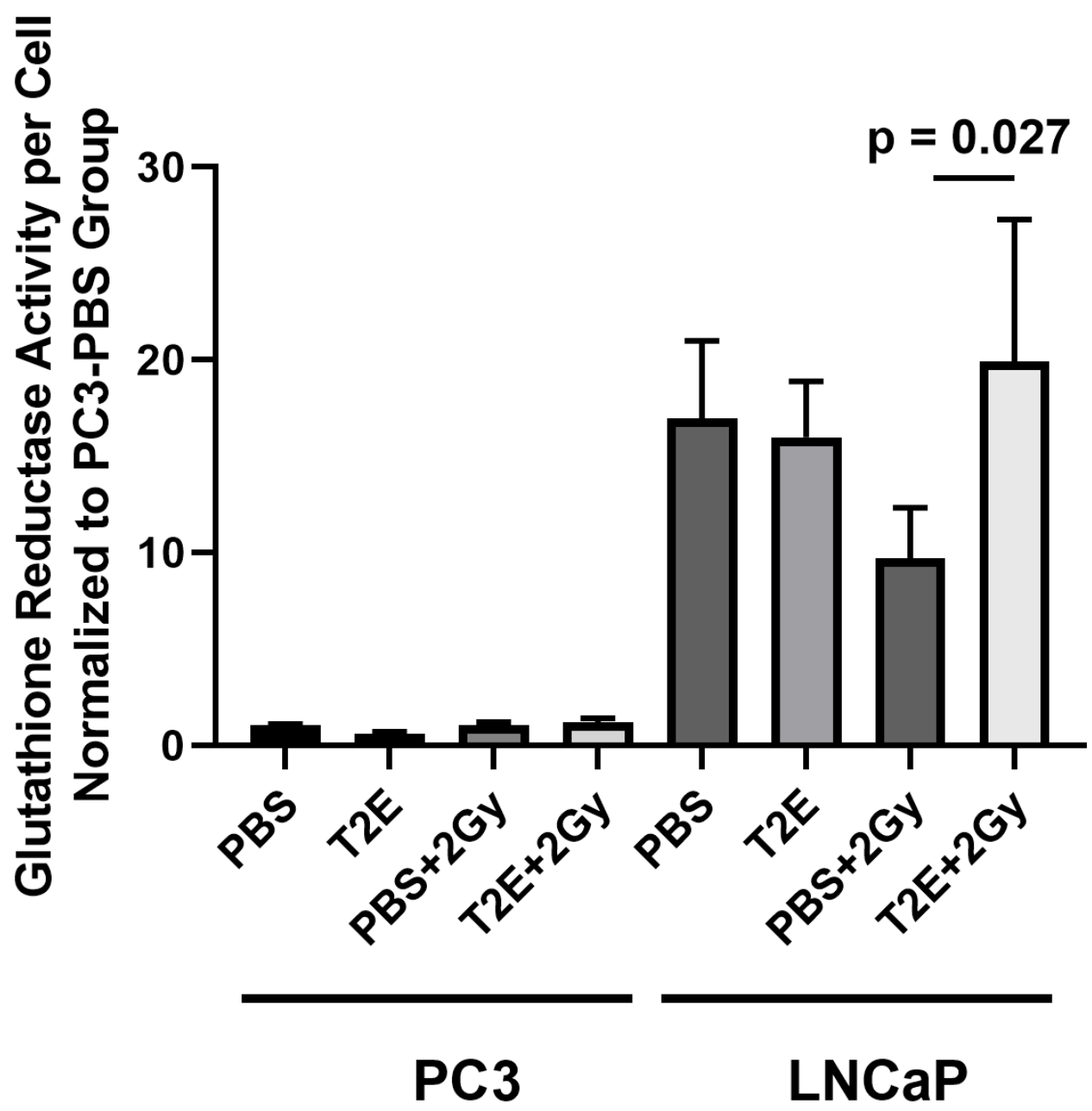


B

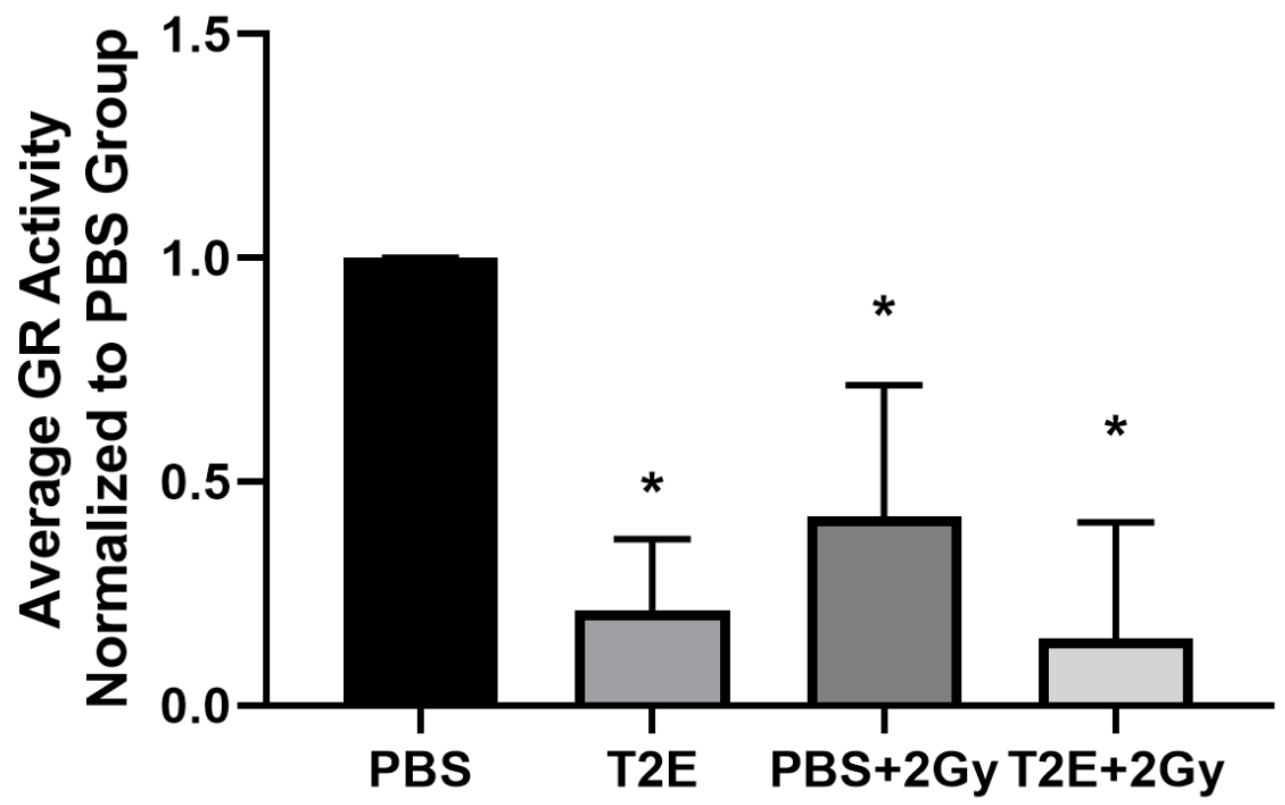




\section{Chapter 4. Discussion and Conclusion}

\section{The mechanisms by which MnTE-2-PyP suppresses prostate cancer growth}

Previous studies, and work from our lab, have shown that MnTE-2-PyP and MnTnBuOE-2-PyP protect normal tissues from radiation damage for prostate cancer treatment during radiotherapy [95-97, 173-175]. Interestingly, our recent work identified that MnTE-2-PyP can further suppress prostate cancer growth and improve radiotherapy both in vivo and in vitro; however, the central mechanism of the inhibitory effects of MnTE2-PyP on prostate cancer growth remains unclear [81, 95].

Mn porphyrins, including MnTE-2-PyP and MnTnBuOE-2-PyP, are designed as SOD mimics that can scavenge superoxide in to $\mathrm{H}_{2} \mathrm{O}_{2}$ [82]. In our studies, we found that MnTE-2-PyP increases cellular $\mathrm{H}_{2} \mathrm{O}_{2}$ levels in multiple prostate cancer cell lines (Figure 6) and inhibited prostate cancer cells growth by $20 \sim 40 \%$, which could be further enhanced when combined with radiation [142]. Many studies have shown that high levels of cellular $\mathrm{H}_{2} \mathrm{O}_{2}$ can result in cell growth arrest or even cell death [176-179]. To determine the role of MnTE-2-PyP-induced $\mathrm{H}_{2} \mathrm{O}_{2}$ in prostate cancer cells, we performed catalase adenovirus transduction in PC3 and LNCaP cells. We found that catalase transduction was able to neutralize the growth inhibitory properties of MnTE-2-PyP in both PC3 and LNCaP cells in a dose-dependent manner (Figure 11, 12); however, transduction of catalase adenovirus did not completely rescue the MnTE-2-PyP-induced cell growth inhibition, even though the cellular $\mathrm{H}_{2} \mathrm{O}_{2}$ levels were recovered back to PBS control levels. In addition, as compared to empty or non-treated cells, catalase transduction alone significantly suppressed the cell proliferation. These results indicate that the $\mathrm{H}_{2} \mathrm{O}_{2}$ levels increase is highly involved in the MnTE-2-PyP-induced prostate cancer cell growth inhibition, but it is not the only mechanism and the cellular redox balance is also important 
for prostate cancer proliferation. Moreover, by using the PEG-catalase compound that only scavenges extracellular $\mathrm{H}_{2} \mathrm{O}_{2}$, we demonstrated that intracellular $\mathrm{H}_{2} \mathrm{O}_{2}$ is the essential factor of prostate cancer cell growth inhibition effects. PEG-catalase is commonly used as a way to scavenge intracellular and extracellular $\mathrm{H}_{2} \mathrm{O}_{2}$ and its effects on cell growth have been widely studied [180-182]; however, in prostate cancer cells PEG-catalase $(1000 \mathrm{U} / \mathrm{ml}, 24$ hours treatment) did not efficiently accumulate inside the cell or exert any intracellular effects on $\mathrm{H}_{2} \mathrm{O}_{2}$ levels (Figure 11G-H). Based on these results, the intracellular $\mathrm{H}_{2} \mathrm{O}_{2}$ induced by MnTE-2-PyP is responsible for the growth inhibitory effects in prostate cancer cells.

Many proteins contain cysteine thiol groups that are sensitive to $\mathrm{H}_{2} \mathrm{O}_{2}$ levels. $\mathrm{H}_{2} \mathrm{O}_{2}$ is able to perform reversible and irreversible modifications on these thiols, which affects protein structures, functions, and stability $[183,184]$. Based on the BIAM and lodoTMTswitch results, we identified that MnTE-2-PyP alone is able to induce cysteine modifications on diverse protein targets that are mainly involved in energy metabolism and protein synthesis.

To determine how MnTE-2-PyP-induced $\mathrm{H}_{2} \mathrm{O}_{2}$ levels contribute to prostate cancer cell growth, we first checked the activity of oxidized protein targets. The lodoTMT-switch assay showed that multiple proteins related to energy metabolism had reversible thiol modifications under MnTE-2-PyP treatment, including ADP/ATP translocase, pyruvate kinase, cytochrome c oxidase subunits, and voltage-dependent anion selective channel (Table 2). Undoubtedly, thiol oxidation could potentially affect the activity of above enzymes or other unidentified targets related to various energy metabolism pathways, which can lead to cell growth inhibition. Moreover, previous studies determined that oxidation of specific cysteine residues, which we identified in the lodoTMT-switch assay, may lead to enzymatic activity decrease, e.g. Cys424 oxidation in pyruvate kinase and 
Cys257 oxidation in ADP/ATP translocase [185, 186]. Unexpectedly, functional analysis of PKM showed that PKM activity did not decrease in PC3 cells with MnTE-2-PyP treatment (Figure 25) and the Seahorse experiments showed that ATP production was not reduced (Figure 28). We also measured the mitochondrial membrane potential because previously we have observed significant lipid peroxidation in prostate cancer cells treated with MnTE-2-PyP and radiation [95]. It is possible that MnTE-2-PyP leads to mitochondrial membrane damage and affects its function, which is commonly reported in cells with mitochondrial dysfunction [187-189]. However, only radiation significantly increased mitochondrial membrane potential in LNCaP cells, and MnTE-2-PyP did not affect mitochondrial membrane potential in PC3 or LNCaP cells (Figure 30B). In addition, increased OXPHOS rates and proton leak was identified in MnTE-2-PyP-treated PC3 and LNCaP cells (Figure 28, 29). These results indicate that MnTE-2-PyP induced a hyperactive and abnormal functioning of mitochondria in prostate cancer cells, but did not substantially damage the ability of mitochondria to produce ATP. We also specifically measured the mitochondrial $\mathrm{H}_{2} \mathrm{O}_{2}$ and ROS levels (Figure 41), but MnTE-2-PyP treatment did not alter these levels, which excludes the possibility that severe mitochondrial membrane damage occurs in MnTE-2-PyP-treated prostate cancer cells and leads to cell growth inhibition. Currently, we do not have direct evidence to explain why MnTE-2-PyP enhanced mitochondrial activity in prostate cancer cells; however, based on the result that cellular ATP levels were not increased in MnTE-2-PyP-treated prostate cancer cells even as OXPHOS rates were enhanced, we assume that the MnTE-2-PyP-induced $\mathrm{H}_{2} \mathrm{O}_{2}$ may trigger a response in prostate cancer cells that consumes large amounts of ATP.

Based on the BIAM assay results, we identified that PP1CB was oxidized and its activity was decreased by 20\% in MnTE-2-PyP-treated PC3 and LNCaP cells (Figure 14 , 15), but its direct downstream target cyclin D1 had opposite phosphorylation states in the 
presence of MnTE-2-PyP in PC3 and LNCaP cells (Figure 17). Interestingly, in both MnTE-2-PyP-treated PC3 and LNCaP cells, we observed dephosphorylation of pRB (Figure 17), a key protein involved in cell cycle progression and has been reported to be under cyclin D1 and protein phosphatase regulation [158-160]. Although pRB hypophosphorylation can lead to cell growth inhibition, we did not observe cell cycle inhibition in PC3 cells in the first 48 hours (Figure 19). It is possible that pRB hypophosphorylation is contributing to prostate cancer cell growth inhibition by affecting a minimal portion of the cellular population, which make them difficult to detect in population analysis.

In addition to protein oxidation, we observed DNA damage in both PC3 and LNCaP cells with MnTE-2-PyP treatment; however, MnTE-2-PyP triggered different responses in these two cell lines. In PC3 cells, MnTE-2-PyP or radiation alone induced nuclear fragmentation, and MnTE-2-PyP combined with radiation showed a maximal nuclear fragmentation. In LNCaP cells, MnTE-2-PyP induced bi-nucleation, but radiation led to nuclear fragmentation and the combination of both treatments did not significantly enhance either type of abnormality (Figure 22B). Interestingly, the catalase transduction did not rescue the nuclear abnormalities in LNCaP cells but mitigated the nuclear fragmentation in PC3 cells (Figure 22C). These results indicate that MnTE-2-PyP-induced $\mathrm{H}_{2} \mathrm{O}_{2}$ levels increase was responsible for enhanced nuclear abnormalities in PC3 cells. And for LNCaP cells, currently we are unable to fully explain the role of $\mathrm{H}_{2} \mathrm{O}_{2}$ levels in nuclear abnormalities due to the fact that catalase transduction alone can scavenge the $\mathrm{H}_{2} \mathrm{O}_{2}$ in LNCaP cells, but also induced cell growth inhibition and bi-nucleation. It is possible that disturbing redox balance by either increasing or decreasing $\mathrm{H}_{2} \mathrm{O}_{2}$ levels in $\mathrm{LNCaP}$ cells could lead to bi-nucleation. 
Moreover, in vivo PC3 tumors treated with 2 Gy/day for 5 days with MnTE-2-PyP displayed enhanced DNA damage as compared to radiotherapy alone as evidenced by YH2AX staining. The nuclear morphology analyses confirmed that MnTE-2-PyP combined with radiation induced more nuclear abnormalities, including multi-nucleation and nuclear fragmentation, but the bi-nucleation was decreased (Figure 23E). This suggests that MnTE-2-PyP may convert bi-nucleation into more genomically unstable states in PC3 tumors during radiotherapy, meanwhile, the different nuclear morphologies in MnTE-2PyP-treated PC3 and LNCaP cell indicate that PC3 cells suffered higher oxidative damage than LNCaP cells. It is possible that the difference between PC3 and LNCaP cells is due to the differences of basal $\mathrm{H}_{2} \mathrm{O}_{2}$ levels or antioxidant defense system capacity. LNCaP cells have higher catalase and GR activity than PC3 cells (Figure 10, 44), and higher $\mathrm{H}_{2} \mathrm{O}_{2}$ levels were more likely to exceed the tolerance of redox balance in PC3 cells and result in synergistic effects with additional ROS produced by radiation exposure.

As we mentioned before, the cell cycle distributions were almost identical in the first 48 hours in PC3 cells treated with or without MnTE-2-PyP, however, we observed a significant increase of Ki67-negative population in both MnTE-2-PyP-treated PC3 and LNCaP cells. As a general proliferation marker and cell quiescence indicator, the increased Ki67-negative population in MnTE-2-PyP-treated prostate cancer cells imply suppressed prostate cancer growth [190, 191]. Consistent with the Ki67 staining results, our previous study showed that there was a significant reduction of PCNA levels in MnTE2-PyP-treated prostate cancer cells with or without radiation [95]. PCNA is a key component in cell cycle progression, especially in S phase for precise DNA replication and DNA damage repair [192-195]. Both Ki67 and PCNA have been used as reliable proliferation markers in immunostaining. Combining the data of nuclear abnormalities, it is possible that the decrease of PCNA expression in prostate cancer cells partially led to 
DNA damage and corresponding nuclear morphology changes, which triggered an increase in Ki67-negative population of cells. Although we have not investigated other proteins that are responsible for DNA repair and cell cycle progression, there was a positive correlation among nuclear abnormalities, Ki67-negative population, and PCNA levels reduction, all of which could ultimately contribute to prostate cancer cell growth inhibition. It is worth mentioning that the total cell growth inhibition was $20 \sim 50 \%$ in MnTE2-PyP-treated PC3 and LNCaP cells as compared to PBS-treated cells, while the total nuclear abnormalities are $3 \sim 12 \%$ in each treated sample. This may explain why we did not observe significant cell cycle alterations in PC3 cells with MnTE-2-PyP treatment, as the relatively small change was not easy to detect statistically. However, this also suggests that the nuclear abnormalities were not the sole mechanism for cell growth inhibition effects in prostate cancer cells.

As discussed above, we found that MnTE-2-PyP resulted in abnormally functioning mitochondria in $\mathrm{PC} 3$ and $\mathrm{LNCaP}$ cells. It is probable that the alterations of $\mathrm{OXPHOS}$ might be linked to the prostate cancer cell growth inhibition. We measured the $N A D(P)+$ and $\mathrm{NAD}(\mathrm{P}) \mathrm{H}$ levels in PC3 and LNCaP cells, because NADH is a central molecule involved in cellular energy metabolism, especially in OXPHOS; while NADPH has a close relation to NADH and plays an important role in cellular redox defense system. We found that both $\mathrm{NAD+/NADH}$ and NADP+/NADPH ratios were significantly enhanced in MnTE-2-PyPtreated PC3 and LNCaP cells, which mainly resulted from decreased levels of NAD(P)H (Figure 32, 33). Currently, we propose two major explanations for $\mathrm{NAD}(\mathrm{P})+$ and $\mathrm{NAD}(\mathrm{P}) \mathrm{H}$ levels changes in MnTE-2-PyP-treated prostate cancer cells.

First of all, the decreased NADPH levels may come from the inhibition of pentose phosphate pathway, based on the results that G6PD and 6PGD activity were both significantly reduced in MnTE-2-PyP-treated prostate cancer cells. And correspondingly, 
the huge reduction of NADPH pool could lead to NADH levels decrease in prostate cancer cells. Previous studies have shown that both enzymes are regulated by acetylation: acetylation on K403 of G6PD will prevent the formation of the functional dimer and reduces G6PD activity, but acetylation on K76 and K294 of 6PGD will increase 6PGD activity [196199]. In normal prostate fibroblast cells, we found that MnTE-2-PyP increased sirtuin proteins activity and affected the MnSOD acetylation states [175]. It is possible that MnTE2-PyP also had similar effects in prostate cancer cells, because we identified increased $\mathrm{NAD}+$ levels in both prostate cancer cells and normal prostate fibroblasts, which can activate sirtuin proteins. However, it is unclear how these enzymes had opposite acetylation state if only sirtuin proteins were activated by MnTE-2-PyP. More acetylatase/deacetylase might be also activated by MnTE-2-PyP that we have not yet identified.

Secondly, the central mechanism of MnTE-2-PyP growth inhibitory effect on prostate cancer cells growth may result from its redox modulator features. Mn porphyrins are called antioxidant SOD mimics as they have catalytic activity of superoxide dismutation reaction and can protect normal tissues from oxidative damage [200-202]. To display its SOD activity, the $\mathrm{Mn}(\mathrm{III})$ center needs to be reduced to the $\mathrm{Mn}$ (II) state by the first superoxide molecule and then be oxidized back to $\mathrm{Mn}(\mathrm{III})$ by the second superoxide molecule to produce $\mathrm{H}_{2} \mathrm{O}_{2}$. The reduction potential of Mn(III)TE-2-PyP/ Mn(II)TE-2-PyP is around $+230 \mathrm{mV}$, which is appropriately in the middle range of the first half reaction $(-160$ $\mathrm{mV}$ vs. $\mathrm{NHE}$ ) and the second half (+890 $\mathrm{mV}$ vs. $\mathrm{NHE})$; thus, performing its SOD activity [203]. Although we showed that MnTE-2-PyP significantly increased $\mathrm{H}_{2} \mathrm{O}_{2}$ levels in multiple prostate cancer cells, due to its relatively low specificity as compared to real SOD proteins, MnTE-2-PyP can potentially react with other intracellular redox-active molecules and induce more effects besides superoxide dismutation. We found that in a PBS system, 
MnTE-2-PyP strongly reacted with the $\mathrm{CMH}$ probe, a probe that forms $\mathrm{CMH}$ radicals with superoxide. This experiment indicates that MnTE-2-PyP can react with other targets besides superoxide. Moreover, early-designed Mn porphyrin compounds have the ability to oxidize common metabolites, including NADH, NADPH, and succinate, in the presence of corresponding enzymes, e.g. complex I, NOS, and complex II [204-206]. In an extreme condition, Mn(III)-TMPyP could efficiently oxidize NADPH when thioredoxin reductase was supplied [207]. Although many of these early experiments were performed in a test tube with excessive addition of Mn porphyrins, and a high concentration of reductants, it is possible that MnTE-2-PyP can directly react with these cellular reductants. The reduction potential of NAD+/NADH and NADP+/NADPH are around $-320 \mathrm{mV}$ and -320 $380 \mathrm{mV}$, respectively, and other major components involved in ETC like CoQ10 ox/ CoQ10 red is around $+100 \mathrm{mV}, \mathrm{Fe}(\mathrm{III})-\mathrm{S} / \mathrm{Fe}(\mathrm{II})-\mathrm{S}$ cluster is around $-250 \mathrm{mV}$, fumarate/succinate is around $+30 \mathrm{mV}$ and $\mathrm{FAD} / \mathrm{FADH}_{2}$ is around $-200 \mathrm{mV}$ [208-213]. We believe that the relatively low specificity and relatively high reduction potential grant MnTE2-PyP the ability to take electrons from original substrates to itself to be reduced to the $\mathrm{Mn}(\mathrm{II})$ state; thus, facilitating the reduction of superoxide to oxygen [211]. In some cases, MnTE-2-PyP may also scavenge $\mathrm{CO}_{3}{ }^{--}$and $\mathrm{ONOO}^{-}$at the cost of $\mathrm{NAD}(\mathrm{P}) \mathrm{H}$ or other major reductants [214]. Currently, we have not proven this occurs in a cellular system, but this could happen.

The second explanation also indicates a new impact of MnTE-2-PyP on prostate cancer cells in addition to protein oxidation, nuclear abnormalities, and enhanced lipid peroxidation with radiation: the depletion of cellular reductants. Importantly, the cellular reductants are not only involved in energy metabolism and redox defense system, but also provides reducing power for anabolic reactions in macromolecule synthesis. For example, NADPH participates in the de novo amino acid synthesis, decrease of NADPH pool may 
affect carbon metabolism in prostate cancer cells and limit the macromolecules production for proper cell division $[135,215]$. Other common reducing metabolites like $\mathrm{FADH}_{2}$ and succinate, will also be consumed by MnTE-2-PyP, and this may explain why prostate cancer cells have increased glucose uptake immediately after MnTE-2-PyP treatment (Figure 31). Based on HPLC results, we did not detected a significant decrease of FADH 2 or succinate pool in MnTE-2-PyP-treated PC3 or LNCaP cells. This could be due to the relatively more positive redox potential of fumarate/succinate $(+30 \mathrm{mV})$ and FAD/FADH $2(-$ $200 \mathrm{mV})$ as compared to $\mathrm{NAD}(\mathrm{P})+/ \mathrm{NAD}(\mathrm{P}) \mathrm{H}(-320 \sim-380 \mathrm{mV})$, thus, MnTE-2-PyP may prefer to react with $\mathrm{NAD}(\mathrm{P}) \mathrm{H}$ first.

Based on above two major explanations, the increased MnTE-2-PyP-induced $\mathrm{H}_{2} \mathrm{O}_{2}$ levels in prostate cancer cells can have two major sources: one from the direct dismutation of superoxide, and the other from the depletion of cellular reducing agents, resulting in accumulation of multiple types of ROS including $\mathrm{H}_{2} \mathrm{O}_{2}$. We believe that the latter is the dominant source because MitoSOX and MitoPY-1 assay results did not show significant alterations of mitochondrial ROS levels or $\mathrm{H}_{2} \mathrm{O}_{2}$ levels.

There are also several other factors that can potentially contribute to the alterations of $\mathrm{NAD}(\mathrm{P})+/ \mathrm{NAD}(\mathrm{P}) \mathrm{H}$ ratios in MnTE-2-PyP-treated prostate cancer cells. First, the decrease of $\mathrm{NADH}$ may result from the increase of OXPHOS in prostate cancer cells, which consumes large amount of NADH and might affect NADPH pools simultaneously [111]; However, based on OXPHOS analysis, the radiation treatment also increased OXPHOS rate (Figure 28, 29), yet we did not observe $\mathrm{NAD}(\mathrm{P}) \mathrm{H}$ alterations in either PC3 or LNCaP cells as compared to PBS control cells. Therefore, this explanation can only partially account for the alterations of $\mathrm{NAD}(\mathrm{P})+$ and $\mathrm{NAD}(\mathrm{P}) \mathrm{H}$ levels. Besides, we also investigated whether NOX enzymes, which consume NADPH to produce $\mathrm{H}_{2} \mathrm{O}_{2}$ and superoxide, contribute to the NADPH levels reduction in MnTE-2-PyP-treated prostate 
cancer cells. We found that the NOX inhibitor, DPI, did not rescue the NADPH pools depletion caused by MnTE-2-PyP treatment. Therefore, NOX enzymes do not deplete the NADPH pool. Moreover, our lab previously reported that MnTE-2-PyP significantly increased the $\mathrm{NAD}(\mathrm{P}) \mathrm{H}$ :quinone acceptor oxidoreductases 1 (NQO1) levels in normal human and mouse prostate fibroblast cells [97]. NQO1 is one of the two major quinone reductases that mediates reduction of quinones to hydroquinones by converting $N A D(P) H$ to $N A D(P)+[97,216]$. We found that increase of NQO1 levels correlated with the activation of NRF2 pathway and mitigated activation of irradiated prostate fibroblast cells in a $\mathrm{H}_{2} \mathrm{O}_{2}$ dependent manner $[97,175]$. it is possible that increased NQO1 levels in PC3 and LNCaP cells could simultaneously decrease the levels of $N A D(P) H$ and increase $N A D(P)+$. While we have detected a significant increase of NQO1 expression levels in LNCaP cell after 48 hours treatment of MnTE-2-PyP, we did not observe a significant increase in NQO1 levels in PC3 cells (data now shown). In addition, the NAD(P)H levels decreased within 6 hours of MnTE-2-PyP treatment but NQO1 levels were not significantly enhanced until 48 hours. Therefore, this is not a likely explanation for why MnTE-2-PyP decreases NADPH levels.

In conclusion, MnTE-2-PyP can increase cellular $\mathrm{H}_{2} \mathrm{O}_{2}$ levels by two different ways: one is the direct dismutation reaction of superoxide molecules, and the other is the cycling reaction initiated with cellular reductants and leading to accumulation of $\mathrm{H}_{2} \mathrm{O}_{2}$ (Figure 45A). The increased $\mathrm{H}_{2} \mathrm{O}_{2}$ levels lead to protein oxidation, DNA damage, and cellular energy metabolism alterations. The increased energy metabolism further accelerates the overall cellular superoxide production and the cycling of MnTE-2-PyP, which induces more protein oxidation and DNA damage. Eventually, a combination of these effects leads to prostate cancer cells growth inhibition (Figure 45B).

In addition, our study also revealed a unique stage of oxidative stress induced by MnTE-2-PyP in prostate cancer cells featured by intracellular ROS levels increase $\left(\mathrm{H}_{2} \mathrm{O}_{2}\right)$, 
protein oxidation, and cell growth inhibition; however, no cell death or mitochondria function compromise was observed. The majority of previous studies have reported severe mitochondrial damage and cell death, such as, apoptosis and necrosis caused by increased intracellular $\mathrm{H}_{2} \mathrm{O}_{2}$ levels [217-220], which result in cell growth inhibition. However, based on our results, we identified a rarely reported condition that mild oxidative stress was induced by MnTE-2-PyP treatment and inhibited prostate cancer cell growth through multiple cellular process including, DNA damage, NAD $(P) H$ depletion, and cell quiescence. Therefore, the stress-induced cell growth inhibition and damage-induced cell growth inhibition may share similarities, such as, protein oxidation and DNA damage, yet the severity may correspond to cellular ROS levels and the cell growth inhibition mechanism may progress from cell quiescence and slowed proliferation to apoptosis and necrosis. 


\section{The combinatorial effects of MnTE-2-PyP and radiation on prostate}

\section{cancer cells.}

MnTE-2-PyP and other similar porphyrin compounds have been tested in radiotherapy as radioprotectors. MnTE-2-PyP protects normal tissues from radiation damage while enhancing radiation-induced tumor growth suppression [81, 95]. Our lab has published several articles addressing the protective effects of MnTE-2-PyP and MnTnBuOE-2-PyP on normal tissue [95, 96, 175]; however, it is still unclear how MnTE2-PyP enhances radiotherapy efficacy on prostate cancer cells.

We found that low dose radiation (2 Gy) did not significantly increase $\mathrm{H}_{2} \mathrm{O}_{2}$ levels in MnTE-2-PyP-treated prostate cancer cells (Figure 6), but when combined with a high dose (20 Gy), $\mathrm{H}_{2} \mathrm{O}_{2}$ levels were increased [142]. In addition, $2 \mathrm{~Gy}$ of radiation induced nuclear fragmentation in both PC3 and LNCaP cells, but MnTE-2-PyP led to nuclear fragmentation in PC3 cells and bi-nucleation in LNCaP cells, the latter which could not be enhanced when combined with radiation. Our early studies also showed that in the presence of radiation, Mn porphyrins (MnTE-2-PyP and MnTnBuOE-2-PyP) increased lipid peroxidation in prostate tumor sections [95]. Radiation provides more radicals that can potentially be scavenged by MnTE-2-PyP to produce more $\mathrm{H}_{2} \mathrm{O}_{2}$ [80]. We believe that the damaging effects of MnTE-2-PyP combined with radiation are dependent on the tolerance of individual cell types and the sensitivity of macromolecules to oxidants. As mentioned before, LNCaP cells have lower basal $\mathrm{H}_{2} \mathrm{O}_{2}$ levels and more antioxidant enzymes/higher activity (e.g. catalase and GR) than PC3 cells, which may relieve the DNA damage and only leads to bi-nucleation; however, in the case of excessive ROS levels, MnTE-2-PyP combined with radiation can indiscriminately lead to nuclear fragmentation and lipid peroxidation in both prostate cancer cells. 
In addition, under low dose radiation (2 Gy), MnTE-2-PyP with radiation did not decrease ATP production. The TMRM data showed that mitochondrial membrane potential were still maintained at control levels in either cell type under radiation and MnTE-2-PyP treatment (Figure 30B). It seems that despite cell growth inhibition effects, MnTE-2-PyP with radiation does not cause massive cell death [221]. The inconsistency and opposite results of some other data, e.g. RNA levels and cellular ATP levels in PC3 and LNCaP cells under MnTE-2-PyP with radiation treatment, indicate that PC3 and LNCaP cells had different responses to the oxidative stress.

Although the effects of MnTE-2-PyP on normal or cancer cells have been studied for years, the reason why it affects normal and cancer cells differently is not completely known. Our lab has identified that MnTE-2-PyP increased the $\mathrm{H}_{2} \mathrm{O}_{2}$ levels in human and mouse prostate fibroblasts, which activates the Keap1-NRF2 and NQO1 signaling pathways. An increase of NAD+ levels were also observed in these cells and this enhanced sirtuin function and enhanced SOD2 activity for detoxification of superoxide during radiation [175]. The activation of the above pathways contribute to the protective effects of MnTE-2-PyP on normal prostate fibroblasts under radiation, which includes a reduction of senescence, alpha-SMA expression, and percentage of abnormal size of cells $[97,175,222]$

It is worth mentioning that $30 \mu \mathrm{M}$ MnTE-2-PyP treatment also led to moderate cell growth inhibition in P3158 cells and a decreasing but statistically insignificant trend in HPrF cells (Figure 5). This seems to be contradictory to the protection effects of MnTE-2PyP on normal cells during radiotherapy; however, currently, the only identified side effect of MnTE-2-PyP in clinical trials is significant blood pressure drop in mice experiments if given at high doses [82]. And we did not observe cell growth inhibition effects of MnTE-2PyP on mouse primary fibroblasts when we isolated from mouse prostates and cultured 
in vitro. We believe that the growth inhibition effects of MnTE-2-PyP on part of the normal cells, like P3158, could be due to the fact that they are immortalized. One explanation for different effects of MnTE-2-PyP on normal and cancer cells is the accumulation of drug inside cells. We found that MnPs accumulated at 2 3 fold higher levels in tumors than normal tissues. Dr. Batinic-Haberle's groups also reported that the MnTE-2-PyP and MnTnBuOE-2-PyP concentration was 10 fold higher in the tumor implanted in the mouse leg as compared to the leg muscle tissue [211]. Another explanation, based on the chemistry of MnTE-2-PyP, is that the slow proliferating cells do not produce high amount of superoxide for fast cycling reactions catalyzed by MnTE-2-PyP, which is the rate-limiting step. This seems to be supported by the doubling time of the cells we used: the PC3 and LNCaP cells have around 24 hours doubling time, P3158 cells are 30 36 hours, HPrF cells are around 60 hours, and PMF cells are around 72 hours. Correspondingly, the PMF cells showed no growth inhibition response to MnTE-2-PyP, and HPrF, P3158, and prostate cancer cells showed increasing growth inhibition response. In addition, the difference of antioxidant defense system and energy metabolism between prostate cancer cells may also contribute to the discrepancy of growth inhibition effects by Mn porphyrins. In normal cells, there is an increase in NAD+ and NADH levels after MnTE-2-PyP treatment [175]. And the overall NAD+/NADH ratios are not altered by MnTE-2-PyP. In cancer cells, the NAD+ levels are enhanced, but NADH levels are greatly reduced, so the $\mathrm{NAD+} / \mathrm{NADH}$ ratios are significantly increased after MnTE-2-PyP treatment. Thus, $\mathrm{NAD+/NADH}$ ratios are affected differently in normal cells vs. cancer cells and this could be why MnTE-2-PyP behaves differently in normal cells as compared to cancer cells. 


\section{Figure 45: The mechanisms by which MnTE-2-PyP suppresses prostate} cancer growth. (A) MnTE-2-PyP can enhance cellular $\mathrm{H}_{2} \mathrm{O}_{2}$ levels through (1) As a SOD mimic, MnTE-2-PyP can directly scavenge superoxide into $\mathrm{H}_{2} \mathrm{O}_{2}$ and thus increase cellular $\mathrm{H}_{2} \mathrm{O}_{2}$ levels (2) MnTE-2-PyP can inhibit pentose phosphate pathway and directly reduce cellular reductants levels, which leads to the NADPH depletion. The depletion of NADPH pool causes dysfunction of cellular antioxidant enzymes, e.g. GR depends on the reducing power of $\mathrm{NADPH}$ to generate $\mathrm{GSH}$ to scavenge $\mathrm{H}_{2} \mathrm{O}_{2}$. The shortage of reducing power for antioxidant defense system results in $\mathrm{H}_{2} \mathrm{O}_{2}$ accumulation. (B) $\mathrm{H}_{2} \mathrm{O}_{2}$ levels increase leads to multiple secondary effects including protein oxidation, DNA damage, and cell quiescence/cell death. Cellular energy metabolism are also altered and may contribute to higher ROS (mainly $\mathrm{H}_{2} \mathrm{O}_{2}$ ) accumulation. These cellular events ultimately lead to prostate cancer cells growth inhibition. In addition, MnTE-2-PyP with radiation can further enhance the strength of above effects and significantly suppress prostate cancer proliferation. 
A

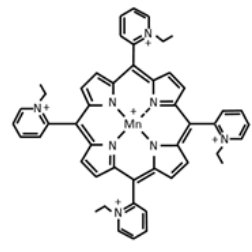

$\mathrm{Mn}$ (III)TE-2-PyP5+

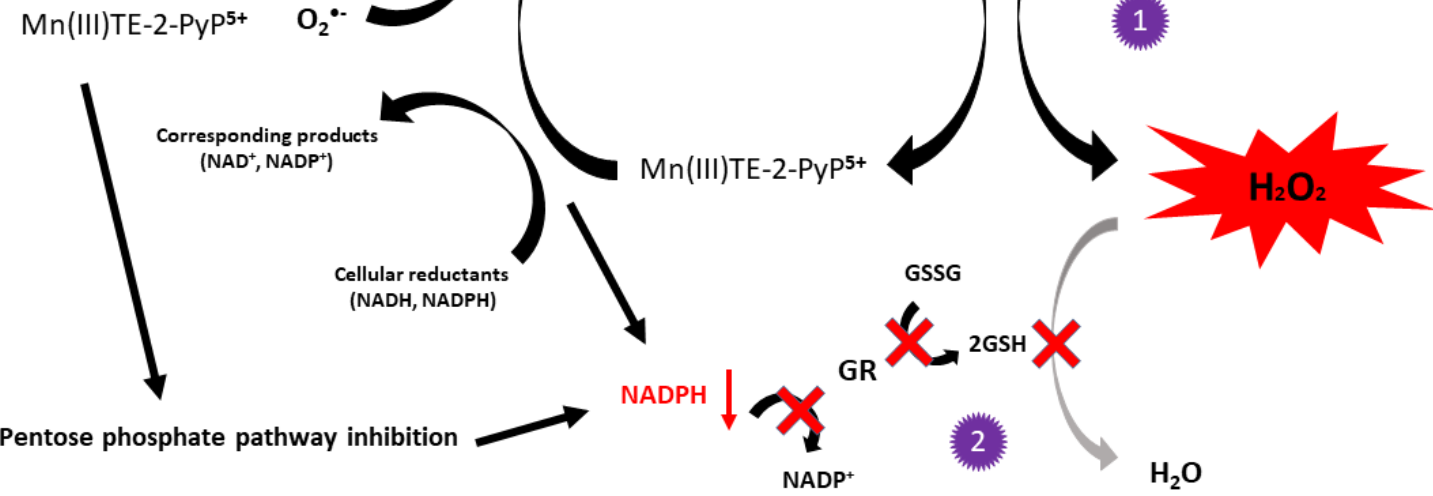

B

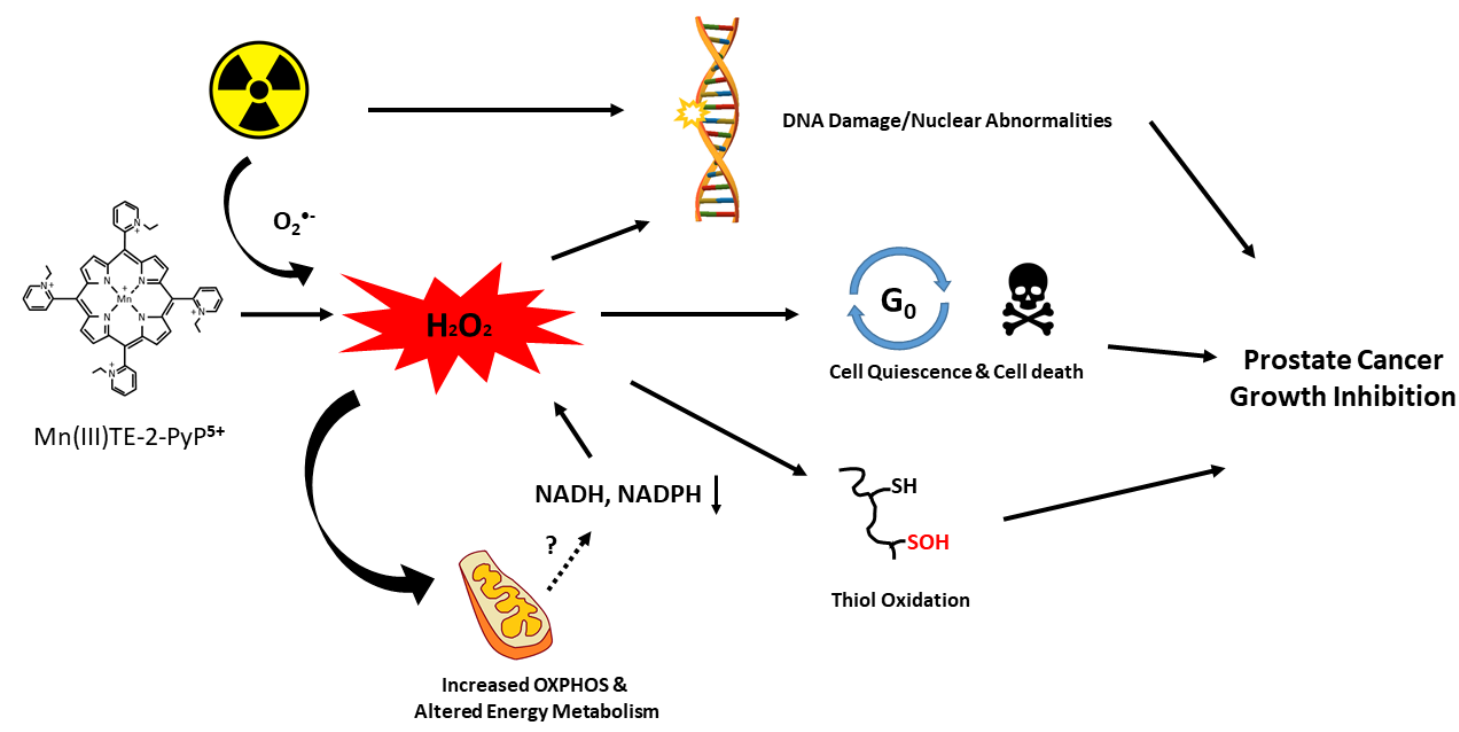




\section{Future directions}

We have shown that MnTE-2-PyP depletes cellular reductants and increases intracellular $\mathrm{H}_{2} \mathrm{O}_{2}$ levels. In addition, we observed protein oxidation and DNA damage in MnTE-2-PyP-treated prostate cancer cells. One potential issue is that we used $30 \mu \mathrm{M}$ MnTE-2-PyP in our experiments, which is not achievable in vivo. Previous studies showed that MnTnBuOE-2-PyP, which is more lipophilic than MnTE-2-PyP and more efficient in radioprotection, had micro molar levels in different tissue types including liver $(\sim 2 \mu \mathrm{M})$, kidney $(\sim 2.5 \mu \mathrm{M})$, peripheral lymph nodes $(4 \sim 6 \mu \mathrm{M})$ based on dog models $[81,223]$. According to another study reported by Dr. Deegan, MnTE-2-PyP administration through i.p. showed around $1 \mu \mathrm{M}$ accumulation in rats tissues, including bowel, prostate, and bladder [173]. As discussed above, we and Dr. Batinic-Haberle found that Mn porphyrins had higher accumulation in tumor cells than normal tissues. We have already shown that lower doses of MnTE-2-PyP $(1 \sim 10 \mu \mathrm{M})$, which is physiologically relevant, can still increase $\mathrm{H}_{2} \mathrm{O}_{2}$ levels and decrease NADPH levels in prostate cancer cells. And based on these dose-dependent effects of MnTnBuOE-2-PyP and MnTE-2-PyP on cellular responses, it is necessary to determine their accumulated concentration in tumor and normal tissues. We are currently trying to use a ${ }^{3} \mathrm{H}$-labeled MnTnBuOE-2-PyP to investigate the distribution of MnTnBuOE-2-PyP in various organs. And future in vitro work should be performed using the corresponding concentrations.

We have found that MnTE-2-PyP treatment significantly altered the cellular redox balance and energy metabolism in prostate cancer cells; however, it is unknown whether MnTE-2-PyP has the same effects on normal prostate cells. Although previous studies have shown that MnTE-2-PyP protects normal tissues but further suppressed prostate tumor growth during radiation. As compared to cancer cells, normal cells usually have lower basal ROS levels and higher antioxidant capacity. The metabolism of normal cells 
are also more regulated than cancer cells, which limits the production of ROS. Therefore, the protein oxidation, DNA damage, and energy metabolism alterations could be secondary effects caused by MnTE-2-PyP treatment and completely dependent on the original redox defense system and metabolic profile of each individual cell type. Since a major application of $\mathrm{Mn}$ porphyrins is radioprotection and $\mathrm{Mn}$ porphyrins will accumulate in both normal tissues and tumors, investigating effects of $\mathrm{Mn}$ porphyrins on normal tissues will promote its application in clinical trials. Currently, we have measured the $\mathrm{H}_{2} \mathrm{O}_{2}$ levels and catalase activity in normal cells, but more work is required on multiple normal cell types to determine how Mn porphyrins behaves differently in normal cells vs. cancer cells during radiotherapy. The NAD(P)+/NAD(P)H levels, GSH/GSSG levels, and GR activity should be measured in P3158 cells, HPrF cells, primary mouse prostate fibroblasts, and prostate epithelial cells with or without MnTE-2-PyP and MnTnBuOE-2PyP.

In addition to $\mathrm{NAD}(\mathrm{P})+$ and $\mathrm{NAD}(\mathrm{P}) \mathrm{H}$ alterations, MnTE-2-PyP may potentially react with other cellular reductants. Since we have observed significant changes of prostate cancer cell energy metabolism, which may result from the depletion of these cellular reductants, we believe that it is necessary to investigate the overall metabolite profile in Mn porphyrin-treated cells. Measuring metabolite changes can provide more information on how MnTE-2-PyP affects cell metabolism globally. We believe that if one or several key metabolites showed significant changes under Mn porphyrins treatment and these changes are unique in prostate cancer as compared to normal tissues, then drugs/therapies targeting that metabolite(s) may improve growth inhibition effect of $\mathrm{Mn}$ porphyrins on prostate cancer cells without increasing radiation or Mn porphyrins doses, which will reduce therapy toxicity. 
One potential application is the combination of Mn porphyrins with chemotherapy. Chemotherapeutic reagent generates high levels of ROS that can induce cell death, and in some cases oxidative damage acts as the primary mechanism for tumor growth suppression effects $[224,225]$. This feature may be utilized by Mn porphyrins as Mn porphyrins can increase $\mathrm{H}_{2} \mathrm{O}_{2}$ levels in cancer cells and, thus, enhance the chemotherapeutic reagent efficacy. We are currently testing the effects of MnTnBuOE-2PyP on prostate cancer cell proliferation in combination with docetaxel. Docetaxel inhibits cell division by preventing microtubule depolymerisation and interfere with chromosome movement [226, 227]. It has been reported that docetaxel can also cause BCL2 phosphorylation and lead to apoptosis in prostate cancer cells [228]. Our preliminary data showed that MnTnBuOE-2-PyP enhanced the growth inhibition effects of docetaxel in PC3 and LNCaP cells. It will be a promising direction as combination of Mn porphyrins can not only increase chemotherapeutic reagents efficacy but may also decrease their toxicity if less reagents are used, or the addition of Mn porphyrins may protect from toxic side effects associated with chemotherapy in normal tissues.

As mentioned in the Introduction, normal prostate epithelial cells have a unique energy metabolism pattern as compared to prostate cancer cells due to the inhibition of TCA cycle by inhibiting mitochondrial aconitase activity and accumulating citrate. Therefore, it is possible to target the TCA cycle or energy production in prostate cancer cells based on another fact that prostate cancer cells require more energy for proliferation. Moreover, combining MnP with a TCA cycle inhibitor or general energy metabolism enzyme may improve therapeutic efficacy. Reagents like 2-DG and CPI-613 have been used in clinical trials and achieved promising results in different tumors [229, 230]. Adding MnTE-2-PyP with these inhibitors may produce better prostate cancer tumor control. 


\section{Appendix}

Appendix A: The application of Mn porphyrins in combination with immunotherapy and radiotherapy for treatment of prostate cancer 


\section{Introduction}

In the last few years, the addition of immunotherapy has been explored as a prostate cancer treatment. The concept of immunotherapy has evolved during the past years since the FDA approval of sipuleucel-T (Provenge), the first immunotherapy agent for hormone-refractory prostate cancer [231]. The development of sipuleucel-T vaccine was based on the action of antigen-presenting cells (APCs), which stimulates T-cell immune response-targeted prostatic acid phosphatase, an antigen that is highly expressed in most prostate cancer cells [231, 232]. Recently, more drugs/therapies have been developed, targeting different immune checkpoints, e.g. CTLA-4-based immunotherapy $[233,234]$ and PD/PDL-based immunotherapy [235-237].

Despite recent progress in immunotherapy, it is unlikely that immunotherapy alone can cure prostate cancer. In one phase-3 trial where 512 metastatic castration-resistant prostate cancer (mCRPC) were involved, the sipuleucel-T treatment only enhanced 4 months of life expectancy in the treated group (25.8 months) as compared to the placebo group (21.7 months). In addition, the cancer progression was not significantly different between two groups of patients [238]. Other types of immunotherapeutic drugs, e.g. Ipilimumab and nivolumab, have been evaluated in clinical trials of mCRPC, but no objective responses were observed [239, 240].

Due to the limited and inconsistent efficacy of mono immunotherapy in prostate cancer treatment, recent studies have focused on combining immunotherapy with other established therapies. Several groups of researches have combined radiotherapy and immunotherapy for prostate cancer patients treatment and achieved promising results [240-242]. The combination of radiotherapy and immunotherapy provides two advantages as compared to mono immunotherapy: 1) radiation-induced cancer cell death can provide the very antigen required for immune response, which enhances tumor-antigenicity and 
prevents antigen tolerance [243]; 2) radiotherapy eliminates a large number of cancer cells acutely, which greatly enhance the treatment efficacy and provides the opportunity for overcoming the complexity of solid tumor microenvironment that has led to failures of traditional immunotherapeutic reagents [244-246]. However, there are still problems to be solved to improve combinatorial therapies. First of all, the radiotherapy has been considered immunosuppressive due to the fact that radiation inevitably damages normal cells including infiltrating immune cells. Although, recent studies showed that radiotherapies might also be immunostimulatory [247-250]. Secondly, adverse effects are commonly reported in immunotherapy in different tumor types, and recently Dr. David Oh's group showed that $\mathrm{mCRPC}$ treated with ipilimumab led to a greater diversification of the T cell repertoire that are highly associated with immunotherapy-related adverse effects $[240,251,252]$. The introduction of radiation may also further increase adverse effects as irrelevant antigens can be overly exposed.

Mn porphyrins may solve above the problems when combined with immunotherapy and radiotherapy successfully. The role of Mn porphyrins on normal tissue protection and tumor suppression during radiotherapy has been extensively evaluated [92-97]. Previous studies show that immune cell activation require ROS signals, indicating that redox signaling pathways are involved in immune response [253]. As a redox modulator, Mn porphyrins may benefit the antitumor response of immune system by activating oxidative stress-mediated signaling pathways. An early study reported that MnTE-2-PyP can affect splenocyte energy metabolism in a type I diabetic model [254]. Moreover, in the context of radiotherapy, MnTE-2-PyP was able to elevate multiple types of immune cells as compared to radiotherapy in the mouse model $[99,100]$. Our recent work also showed that MnTE-2-PyP enhances the Th1 cell population in both spleen and lymph nodes during radiotherapy (data not shown). We also found that MnTE-2-PyP could down-regulated 
Th2 population. We believe that Mn porphyrins can potentially lead to tumor killing and enhance tumor antigenicity during radiotherapy, while, immune cells could be protected from radiation damage. However, more information is required to support this assumption and to better understand the relation of MnTE-2-PyP and both immunotherapy and radiotherapy. 


\section{Materials and methods}

\section{A1.1. Cell culture of PC3-Luc and RM1 tumor cells}

The constitutive luciferase expressing PC3 cells (PC3-Luc) were purchased from Applied Biological Materials Inc. (Richmond, BC, Canada). For in vitro cell culture, cells were maintained in RPMI-1640 medium with the addition of $10 \%$ fetal bovine serum (FBS) and $1 \%$ penicillin/streptomycin.

\section{A1.2. Animal husbandry}

Male C57BI/6 mice (Charles River Laboratories, Wilmington, MA, USA and Taconic, Rensselaer, NY, USA) and athymic nude mice (Charles River Laboratories) were used for all experiments. Mice were exposed to a 12 hours light/12 hours dark cycle and fed and watered ad libitum at the University of Nebraska Medical Center (UNMC, Omaha, NE, USA). All experimental protocols were reviewed and approved by the UNMC Institutional Animal Care and Use Committee (20-019-03FC).

\section{A1.3. Orthotopic implantation of PC3 and RM1 tumor cells}

Sources of the experimental instrumental have been listed in Chapter 2. Materials and methods-2.3. Orthotopic implantation of PC3 and RM1 tumor cells section. For PC3Luc athymic nude mice were used and for RM1 tumor implantation, C57BI/6 mice were used. Mice were anesthetized by continuous flow of $2.5 \%$ isoflurane with oxygen using a mouse anesthesia machine. A mixture of PC3-Luc cells or RM1 cells and Matrigel was injected into the dorsal prostatic lobe $(50 \mu \mathrm{L}$ mixture containing 2 million PC3-Luc or 1 million RM1 cells). The peritonium was closed with absorbable catgut sutures and the skin was closed with wound clips. Then, 0, 6, 24, and 48 hours after surgery, buprenorphine $(0.1 \mathrm{mg} / \mathrm{kg})$ was administrated intraperitoneally. The health condition of all mice was monitored daily, and 10 days after surgery, the wound clips were removed. 


\section{A1.4. Radiotherapy protocol for mice}

The procedure for animal radiotherapy has been previously described in Chapter 2. Materials and methods-2.4. Radiotherapy protocol for mice section.

PC3 tumor size was measured every week after the 5th week of orthotopic implantation by intraperitoneal D-Luciferin injection (100 mg/kg) and Xenogen IVIS Spectrum bioluminescence imaging system. Tumor sizes of each mouse were compared by calculating the regions of interest (ROI) based on luminescence intensity.

RM1 tumors were CT imaged to verify size (tumor size $=$ width $\times$ length $\times$ height) and location, then irradiated with image-guided X-rays using the Small Animal Radiation Research Platform.

For both PC3 and RM1 tumor-bearing mice, mice with extremely small or large tumors were excluded from the experiment. For PC3 (five weeks post-surgery) and RM1 tumors (three weeks post-surgery), mice were divided into 2 groups: PBS or MnTE-2-PyP with radiation (2 Gy per day, for five sequential days). PBS or MnTE-2-PyP ( $5 \mathrm{mg} / \mathrm{kg}$ ) was administrated intraperitoneally 24 hours before radiation and three times every week until mice were sacrificed.

\section{A1.5. Tumor harvesting and tumor size measurement}

The procedure for tumor harvesting has been previously described in Chapter 2. Materials and methods-2.5. Tumor harvesting and tumor size measurement section. In brief, animals were sacrificed 2 weeks post-radiation. The width and length of the excised tumor were measured with calipers and the volume was estimated according to the formula: $\left[(\text { width })^{2} \times\right.$ length/2]. The tumor was fixed in $4 \%$ formalin followed by $70 \%$ ethanol, and these tissues were paraffin embedded. Sections were cut and placed on slides for immunostaining. 


\section{A1.6. Immunohistochemical staining for in vivo samples}

Tissue sections were deparaffinized in histoclear and rehydrated in graded alcohols $(100 \%, 95 \%, 70 \%)$, then rinsed in running deionized water. For antigen retrieval, $10 \mathrm{mM} \mathrm{Na}$-Citrate ( $\mathrm{pH}$ 6.0, for CD68, CD3)/EDTA Tris ( $\mathrm{pH}=9$, for CD4, CD8) was boiled on a hotplate, and slides containing tumor sections were boiled in corresponding buffer for $20 \mathrm{~min}$. The slides were then cooled for $30 \mathrm{~min}$ at room temperature and washed twice with PBS. In some conditions, endogenous peroxidases were quenched by $3 \% \mathrm{H}_{2} \mathrm{O}_{2}$ solution for $5 \mathrm{~min}$.

The PK6100 standard ABC kit (Vector, Burlingame, CA, USA) was used for blocking and probing. In brief, sections were blocked by $2 \%$ BSA (For CD4) or $10 \%$ goat serum (For CD8, CD68) and incubated in humidified chamber for $60 \mathrm{~min}$ at room temperature. The primary antibody treatment (CD4-1:1000, CD8-1:2000, CD68-1:100) was applied for 16 hours at $4{ }^{\circ} \mathrm{C}$. Then the biotinylated secondary antibody (from ABC kit, $5 \%$ in corresponding blocking buffer) was used for $30 \mathrm{~min}$ at room temperature. After washing, the Vectastain $A B C$ Reagent was used for $30 \mathrm{~min}$ at room temperature.

The DAB substrate (Vector, Burlingame, CA, USA) was used for peroxidase detection. Sections were covered in DAB substrate for 2 5 min until desired staining was achieved. The reaction was then stopped by washing with PBS three times. For counterstaining, sections were stained with hematoxylin for 10 seconds and washed with $\mathrm{ddH}_{2} \mathrm{O}$. Slides were dehydrated through graded alcohols $(95 \%, 100 \%)$, cleared in histoclear and mounted with Cytoseal 60 and sealed with cover slips. The sections were observed under LEICA DM4000 B LED fluorescent microscope as described above. 


\section{Results}

\section{A2.1. Radiation increases macrophage infiltration in RM1 tumor samples}

RM1 are an aggressive mouse prostate cancer cell that form tumors and metastasize in C57BI/6 mice. We have shown that MnTE-2-PyP increased $\mathrm{H}_{2} \mathrm{O}_{2}$ levels in aggressive prostate cancer cells lines, including RM1 cells (Figure 6). To determine the role of MnPs in immune system activation, we investigated the role of MnTnBuOE-2-PyP in RM1-tumor implanted C57BI/6 mice during radiotherapy (10 Gy total). We measured the area of tumor samples that were infiltrated with macrophages, as indicated by CD68 positive staining. We found that radiotherapy significantly enhanced macrophage infiltration in tumor samples. However, MnTnBuOE-2-PyP treatment did not further increase macrophage infiltration area as compared to PBS with radiation treatment (Figure 46). 
Figure 46: Radiation increases macrophage infiltration in RM1 tumor samples. Tumor sections were stained with CD68 to indicate the macrophage infiltration region. For quantification, a total of 10 images were taken from each sample and the infiltrated area was measured by ImageJ. The macrophage infiltration area were calculated and compared among different conditions. $n=2$ for PBS alone, $n=5$ for PBS + radiation, $n=6$ for MnTnBuOE-2-PyP + radiation. * $p<0.05$ compared to PBS treatment. 


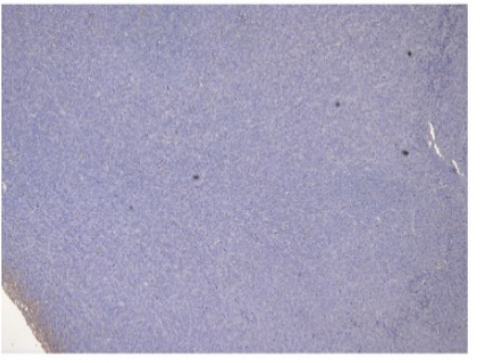

PBS

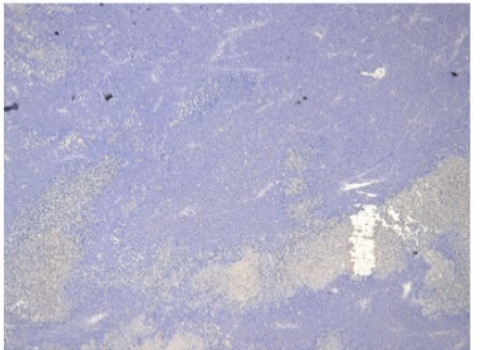

PBS + 10Gy

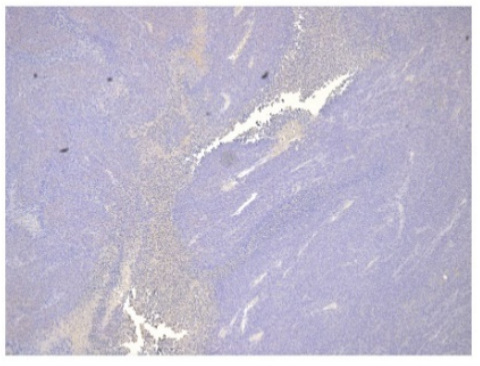

BuOE + 10Gy

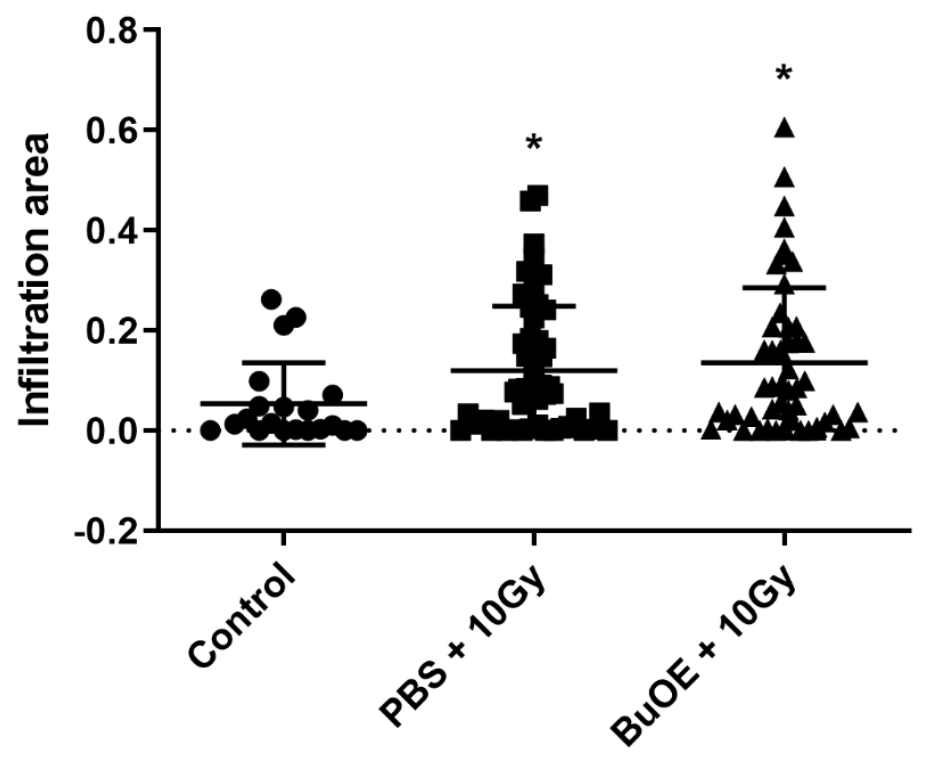




\section{A2.2. MnTnBuOE-2-PyP reduces immune cells in RM1 tumor after radiotherapy}

To investigate the potential of combining immunotherapy with $\mathrm{MnPs}$ and radiotherapy, the C57BL/6 mice were implanted orthotopically with RM1 mouse prostate tumors and then underwent $8 \mathrm{~Gy}$ irradiation treatment. We found that 2 weeks after radiotherapy, there was a decreasing trend of CD4, CD8, CD68 cells in MnTnBuOE-2PyP + radiation samples as compared to PBS + radiation. However, the necrotic area in the tumors was increased with MnTnBuOE-2-PyP + radiation treatment, but this trend was not significantly different (Figure 47).

Although we did not observe effects in the size of primary tumor sites, we found that there was a significant decrease of metastasis in MnTnBuOE-2-PyP and radiation group as compared to radiation alone group (Figure 48). Therefore, it is possible that MnTnBuOE-2-PyP increased the radiotherapy efficacy and reduced the RM1 aggressiveness, which is consistent with the increase of tumor necrosis; however, the sample sizes and timing of mice sacrifice might needed to be adjusted to better

understand the reason why immune cells population decreased. In addition, the increase of tumor necrosis may provide more antigen presentation for immunotherapy. 
Figure 47: MnTnBuOE-2-PyP reduces immune cell population in RM1 tumor after radiotherapy. Tumor sections were stained for CD4 (T helper cell), CD8 (cytotoxic T cell), and CD68 (macrophage). A total of 10 images were taken from each sample and the average number of staining positive cells were calculated. The necrosis was identified in each image of CD4, CD8, and CD68 staining based on the criteria that significant RM1 cell death and missing tumor area are observed. $n=4$ for PBS + radiation, $n=3$ for MnTnBuOE-2-PyP + radiation. 
A

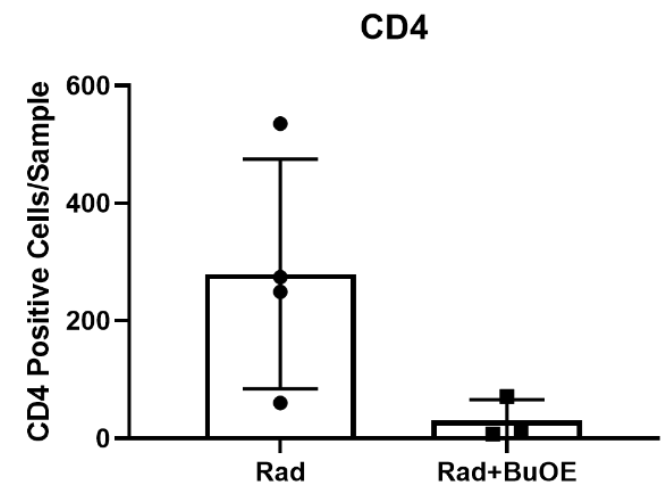

C

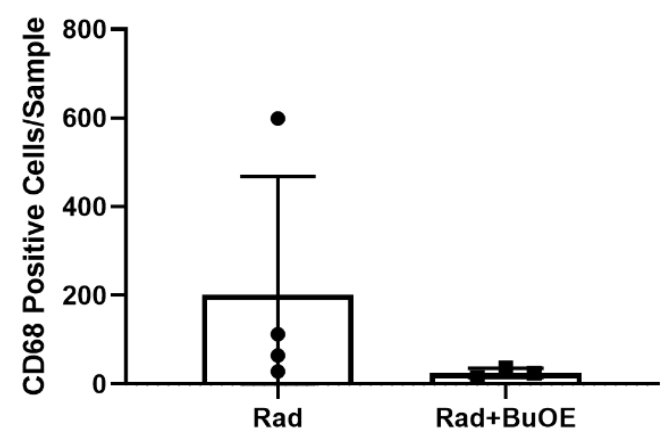

B

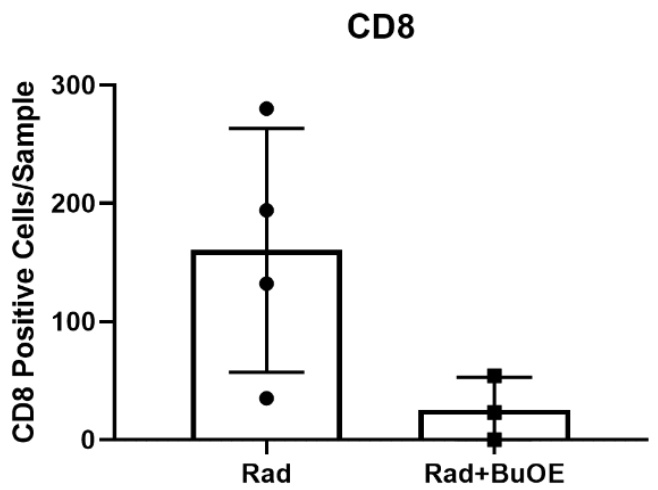

D

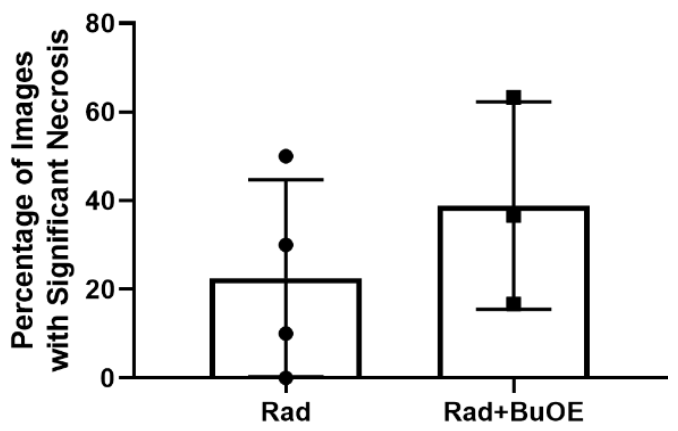


Figure 48: MnTnBuOE-2-PyP greatly reduces metastasis in RM1 tumor after radiotherapy. Tumor volume, weight, growth rate, and metastasis sites were compared between PBS + radiation and MnTnBuOE-2-PyP + radiation RM1 tumor models. $n=18$ for PBS + radiation, $\mathrm{n}=19$ for MnTnBuOE-2-PyP + radiation. 


\section{RM1-Tumor Volume}

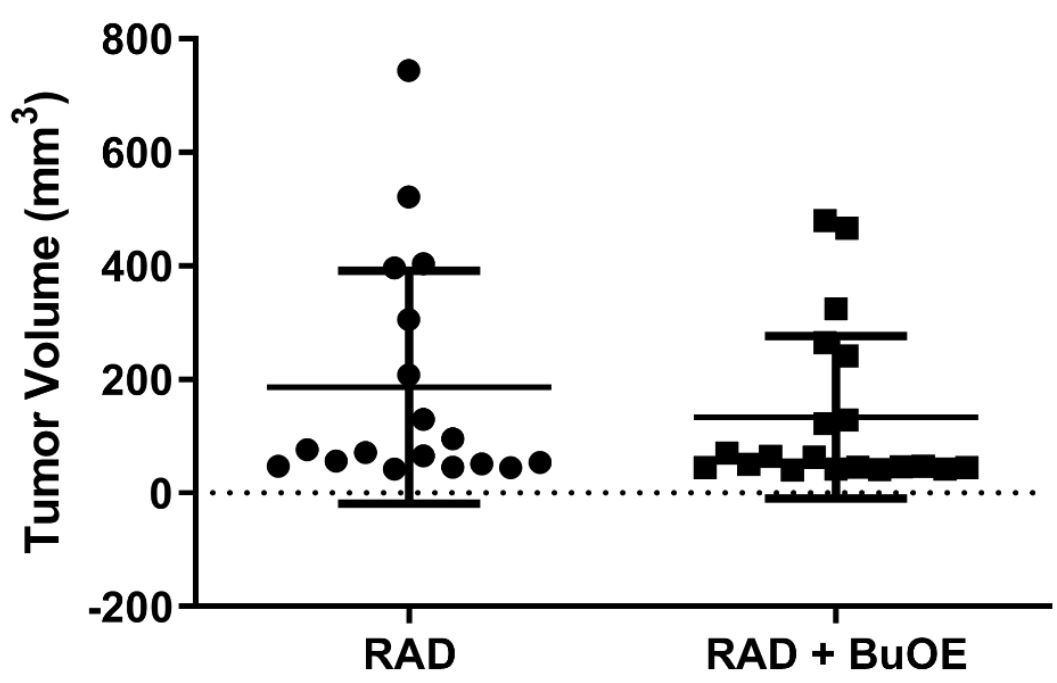

RM1-Metastasis

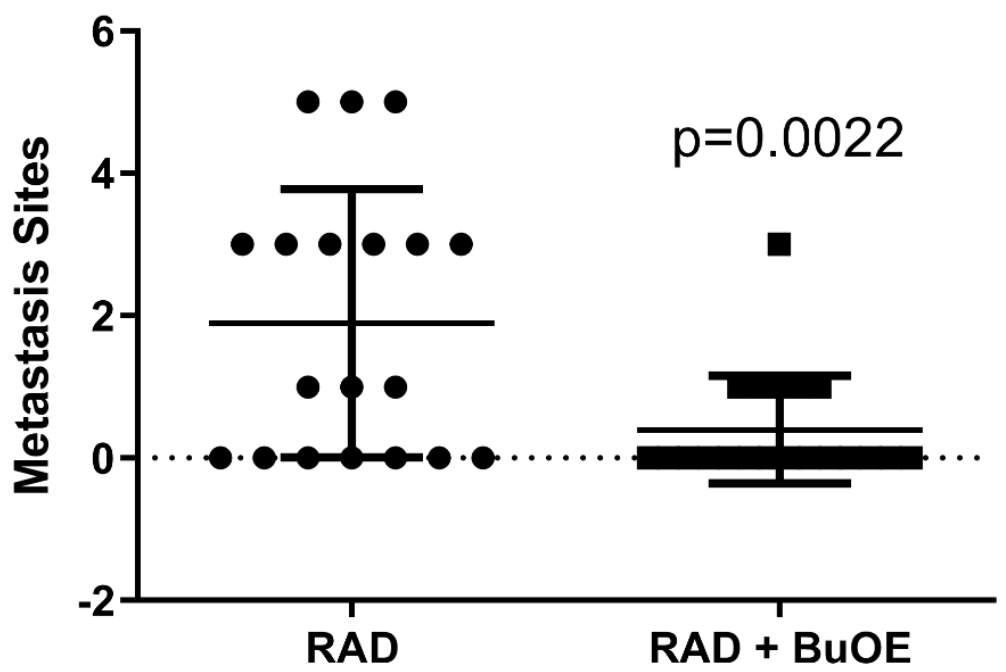




\section{A2.3. MnTE-2-PyP increases macrophage infiltration in PC3 tumors}

We also investigated the effects of MnTE-2-PyP in PC3-tumor implanted mice. We measured the average numbers of macrophages that infiltrated into the PC3 tumors, as indicated by CD68 positive staining. We found that MnTE-2-PyP combined with radiation significantly enhanced the macrophage infiltration in PC3 tumor samples as compared to radiation alone (Figure 49). As compared to the A2.1 results, we performed this experiment in nude mice which does not have intact immune system and the $\mathrm{Mn}$ porphyrins were different, both of which may contribute to the different results between two models. We have observed longer lifespan and more reduction of tumor volumes in the presence of MnTE-2-PyP as compared to radiation alone, and it is possible that MnTE2-PyP enhanced radiotherapy efficacy in PC3 tumor models, while the samples were at the stage when immune cells were destroying PC3 tumor cells, thus, we observed increased macrophage staining. 
Figure 49: MnTE-2-PyP increases macrophage infiltration in PC3 tumors. Tumor sections were stained with CD68 (macrophage). A total of 10 images were taken from each sample and the average number of staining positive cells were calculated. $n=4$ for $\mathrm{PBS}+$ radiation, $\mathrm{n}=4$ for MnTE-2-PyP + radiation 

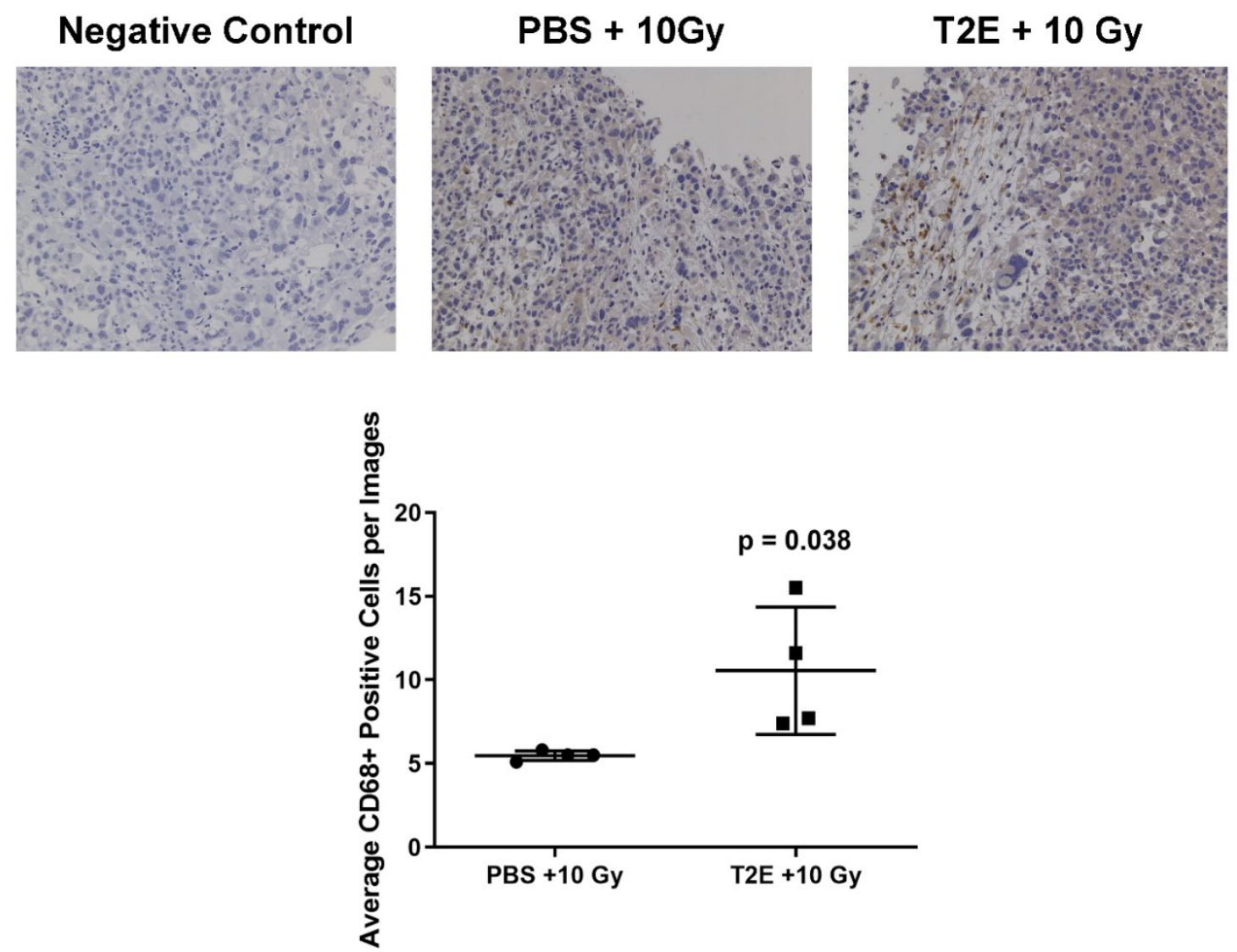


\section{Discussion and Conclusion}

Immunotherapies have thrived as novel treatments due to their success in improving survival rates of patients with certain cancers [255]; however, immunotherapy relies on the effective recognition of cancer cells, which is a limitation of many solid tumors, including prostate cancer [256].

Based on the PC3 and RM1 tumor models, we found that MnTE-2-PyP or radiation significantly increased the immune cell infiltration in the tumor region, which is desired for the combination of MnPs, immunotherapy, and radiotherapy. Although MnTnBuOE-2-PyP with radiation ( $8 \mathrm{~Gy}$ ) showed less infiltration after 2 weeks treatment time points as compared to PBS with radiation, the necrosis area was enhanced and metastasis was suppressed. Due to the fact that only large sized tumors were accessible for immunostaining, it is possible that the decreased immune cells infiltration did not truly reflect the majority of other samples. Therefore, we believe that MnTnBuOE-2-PyP treatment actually improved radiation effects and accelerated the immune system clearance of RM1 tumor cells. The similar experiment performed using a PC3 tumor model, with MnTE-2-PyP treatment, showed that MnTE-2-PyP significantly increased macrophage infiltration at 2-weeks time post-radiation. It is possible that MnTE-2-PyP is less lipophilic and potent than MnTnBuOE-2-PyP, which takes longer to improve immune system capacity. In addition, the difference of immune system between the animal models (PC3: athymic nude mice; RM1: C57BL/6J mice) may contribute to this discrepancy.

The increase of necrosis by MnTnBuOE-2-PyP in RM1 model can be beneficial for immunotherapy, since radiation-induced cancer cell death can provide the antigen required for immune response. This concept has been supported by many investigators who have combined immunotherapy and radiotherapy, which could be further enhanced by MnPs treatment [257-259]. 
In addition to the above promising effects, our lab also found that MnTE-2-PyP treatment enhanced the Type 1 helper T cells: Type 2 helper $T$ (Th1:Th2) ratio in splenocytes, especially during radiation, and in lymphocytes, radiation enhanced the cells Th1:Th2 ratio and this was further elevated in combination with MnTE-2-PyP (data not shown). Helper T (Th) cells are critical in anti-tumor response by providing signals required for CD8+ cells-mediated cytotoxic effects [260, 261], and the Th1:Th2 balance has been used to predict/illustrate the progress for immune response patterns [262]. Similar results were reported by Dr. Paiboon Jungsuwadee who identified that MnTE-2-PyP suppressed Th2 cell proliferation in vitro co-culturing system with dendritic cells, which was probably through ROS-related pathways [263].

The fact that MnTE-2-PyP could alter Th1 and Th2 cells population in the nontumor animal models indicate that MnPs can affect immune system independently. Therefore, MnPs may act as a bridge between immunotherapy and radiotherapy. This will be the focus of future studies. 


\section{Reference}

1. Rebbeck, T.R., Prostate Cancer Genetics: Variation by Race, Ethnicity, and Geography. Semin Radiat Oncol, 2017. 27(1): p. 3-10.

2. Siegel, R.L., K.D. Miller, and A. Jemal, Cancer statistics, 2019. CA Cancer J Clin, 2019. 69(1): p. 7-34.

3. Grozescu, T. and F. Popa, Prostate cancer between prognosis and adequate/proper therapy. J Med Life, 2017. 10(1): p. 5-12.

4. Taplin, M.E., et al., Mutation of the androgen-receptor gene in metastatic androgen-independent prostate cancer. N Engl J Med, 1995. 332(21): p. 1393-8.

5. Beltran, H., et al., Emerging Molecular Biomarkers in Advanced Prostate Cancer: Translation to the Clinic. Am Soc Clin Oncol Educ Book, 2016. 35: p. 131-41.

6. Fraser, M., et al., Genomic, pathological, and clinical heterogeneity as drivers of personalized medicine in prostate cancer. Urol Oncol, 2015. 33(2): p. 85-94.

7. Mateo, J., et al., DNA-Repair Defects and Olaparib in Metastatic Prostate Cancer. N Engl J Med, 2015. 373(18): p. 1697-708.

8. Bose, R., et al., ERF mutations reveal a balance of ETS factors controlling prostate oncogenesis. Nature, 2017. 546(7660): p. 671-675.

9. $\mathrm{Mu}, \mathrm{P}$. , et al., SOX2 promotes lineage plasticity and antiandrogen resistance in TP53- and RB1-deficient prostate cancer. Science, 2017. 355(6320): p. 84-88.

10. Jamaspishvili, T., et al., Clinical implications of PTEN loss in prostate cancer. Nat Rev Urol, 2018. 15(4): p. 222-234.

11. Vlietstra, R.J., et al., Frequent inactivation of PTEN in prostate cancer cell lines and xenografts. Cancer Res, 1998. 58(13): p. 2720-3.

12. Carm, K.T., et al., Interfocal heterogeneity challenges the clinical usefulness of molecular classification of primary prostate cancer. Sci Rep, 2019. 9(1): p. 13579.

13. Wang, G., et al., Genetics and biology of prostate cancer. Genes Dev, 2018. 32(17-18): p. 1105-1140.

14. Powell, I.J., et al., Should African-American men be tested for prostate carcinoma at an earlier age than white men? Cancer, 1999. 85(2): p. 472-7.

15. Henley, S.J., et al., Annual report to the nation on the status of cancer, part I: National cancer statistics. Cancer, 2020. 126(10): p. 2225-2249.

16. Dobbs, R.W., et al., Prostate cancer disparities in Hispanics by country of origin: a nationwide population-based analysis. Prostate Cancer Prostatic Dis, 2019. 22(1): p. 159-167.

17. Evans, A.J., Treatment effects in prostate cancer. Mod Pathol, 2018. 31(S1): p. S110-121.

18. Ritch, C. and M. Cookson, Recent trends in the management of advanced prostate cancer. F1000Res, 2018. 7.

19. Karantanos, T., et al., Understanding the mechanisms of androgen deprivation resistance in prostate cancer at the molecular level. Eur Urol, 2015. 67(3): p. 4709.

20. Dal Pra, A., et al., Contemporary role of postoperative radiotherapy for prostate cancer. Transl Androl Urol, 2018. 7(3): p. 399-413.

21. Santivasi, W.L. and F. Xia, lonizing radiation-induced DNA damage, response, and repair. Antioxid Redox Signal, 2014. 21(2): p. 251-9.

22. Labbe, M., et al., microRNAs identified in prostate cancer: Correlative studies on response to ionizing radiation. Mol Cancer, 2020. 19(1): p. 63. 
23. Kim, J., J.S. Park, and W.S. Ham, The role of metastasis-directed therapy and local therapy of the primary tumor in the management of oligometastatic prostate cancer. Investig Clin Urol, 2017. 58(5): p. 307-316.

24. Artibani, W., et al., Management of Biochemical Recurrence after Primary Curative Treatment for Prostate Cancer: A Review. Urol Int, 2018. 100(3): p. 251-262.

25. Chaiswing, L., et al., Profiles of Radioresistance Mechanisms in Prostate Cancer. Crit Rev Oncog, 2018. 23(1-2): p. 39-67.

26. Mun, G.I., et al., Pharmacology of natural radioprotectors. Arch Pharm Res, 2018. 41(11): p. 1033-1050.

27. Yartsev, S. and G. Bauman, Target margins in radiotherapy of prostate cancer. $\mathrm{Br}$ J Radiol, 2016. 89(1067): p. 20160312.

28. Bohmer, D., et al., Radiotherapy and Hormone Treatment in Prostate Cancer. Dtsch Arztebl Int, 2016. 113(14): p. 235-41.

29. Bilusic, M., R.A. Madan, and J.L. Gulley, Immunotherapy of Prostate Cancer: Facts and Hopes. Clin Cancer Res, 2017. 23(22): p. 6764-6770.

30. Sies, H. and E. Cadenas, Oxidative stress: damage to intact cells and organs. Philos Trans R Soc Lond B Biol Sci, 1985. 311(1152): p. 617-31.

31. Cadenas, E. and H. Sies, Oxidative stress: excited oxygen species and enzyme activity. Adv Enzyme Regul, 1985. 23: p. 217-37.

32. Pizzino, G., et al., Oxidative Stress: Harms and Benefits for Human Health. Oxid Med Cell Longev, 2017. 2017: p. 8416763.

33. Pham-Huy, L.A., H. He, and C. Pham-Huy, Free radicals, antioxidants in disease and health. Int J Biomed Sci, 2008. 4(2): p. 89-96.

34. Genestra, M., Oxyl radicals, redox-sensitive signalling cascades and antioxidants. Cell Signal, 2007. 19(9): p. 1807-19.

35. Droge, W., Free radicals in the physiological control of cell function. Physiol Rev, 2002. 82(1): p. 47-95.

36. Conrad, M., et al., Regulation of lipid peroxidation and ferroptosis in diverse species. Genes Dev, 2018. 32(9-10): p. 602-619.

37. Forman, H.J., et al., Protein cysteine oxidation in redox signaling: Caveats on sulfenic acid detection and quantification. Arch Biochem Biophys, 2017. 617: p. 26-37.

38. Nishida, N., et al., Reactive oxygen species induce epigenetic instability through the formation of 8-hydroxydeoxyguanosine in human hepatocarcinogenesis. Dig Dis, 2013. 31(5-6): p. 459-66.

39. Tonnies, E. and E. Trushina, Oxidative Stress, Synaptic Dysfunction, and Alzheimer's Disease. J Alzheimers Dis, 2017. 57(4): p. 1105-1121.

40. Pignatelli, P., et al., Oxidative stress and cardiovascular disease: new insights. Kardiol Pol, 2018. 76(4): p. 713-722.

41. Oh, B., et al., Oxidative stress in prostate cancer patients: A systematic review of case control studies. Prostate Int, 2016. 4(3): p. 71-87.

42. Case, A.J., On the Origin of Superoxide Dismutase: An Evolutionary Perspective of Superoxide-Mediated Redox Signaling. Antioxidants (Basel), 2017. 6(4).

43. Wang, Y., et al., Superoxide dismutases: Dual roles in controlling ROS damage and regulating ROS signaling. J Cell Biol, 2018. 217(6): p. 1915-1928.

44. Miao, L. and D.K. St Clair, Regulation of superoxide dismutase genes: implications in disease. Free Radic Biol Med, 2009. 47(4): p. 344-56.

45. Okado-Matsumoto, A. and I. Fridovich, Subcellular distribution of superoxide dismutases (SOD) in rat liver: Cu,Zn-SOD in mitochondria. J Biol Chem, 2001. 276(42): p. 38388-93. 
46. Fukai, T., et al., Extracellular superoxide dismutase and cardiovascular disease. Cardiovasc Res, 2002. 55(2): p. 239-49.

47. Marklund, S.L., Extracellular superoxide dismutase and other superoxide dismutase isoenzymes in tissues from nine mammalian species. Biochem J, 1984. 222(3): p. 649-55.

48. Karnati, S., et al., Mammalian SOD2 is exclusively located in mitochondria and not present in peroxisomes. Histochem Cell Biol, 2013. 140(2): p. 105-17.

49. Surapaneni, K.M. and G.R. Venkata, Lipid peroxidation and antioxidant status in patients with carcinoma of prostate. Indian J Physiol Pharmacol, 2006. 50(4): p. 350-4.

50. Aydin, A., et al., Oxidative stress and antioxidant status in non-metastatic prostate cancer and benign prostatic hyperplasia. Clin Biochem, 2006. 39(2): p. 176-9.

51. Plymate, S.R., et al., Increased manganese superoxide dismutase (SOD-2) is part of the mechanism for prostate tumor suppression by Mac25/insulin-like growth factor binding-protein-related protein-1. Oncogene, 2003. 22(7): p. 1024-34.

52. Zhong, W., et al., Suppression of the malignant phenotype of human glioma cells by overexpression of manganese superoxide dismutase. Oncogene, 1997. 14(4): p. 481-90.

53. Kim, J., et al., SOD3 acts as a tumor suppressor in PC-3 prostate cancer cells via hydrogen peroxide accumulation. Anticancer Res, 2014. 34(6): p. 2821-31.

54. von Ossowski, I., G. Hausner, and P.C. Loewen, Molecular evolutionary analysis based on the amino acid sequence of catalase. J Mol Evol, 1993. 37(1): p. 71-6.

55. Nandi, A., et al., Role of Catalase in Oxidative Stress- and Age-Associated Degenerative Diseases. Oxid Med Cell Longev, 2019. 2019: p. 9613090.

56. Iliukha, V.A., [Superoxide dismutase and catalase in organs of mammalians with various ecological genesis]. Zh Evol Biokhim Fiziol, 2001. 37(3): p. 183-6.

57. Bai, J. and A.I. Cederbaum, Mitochondrial catalase and oxidative injury. Biol Signals Recept, 2001. 10(3-4): p. 189-99.

58. Heck, D.E., et al., Mechanisms of oxidant generation by catalase. Ann N Y Acad Sci, 2010. 1203: p. 120-5.

59. Makino, N., et al., Kinetic studies on the removal of extracellular hydrogen peroxide by cultured fibroblasts. J Biol Chem, 1994. 269(2): p. 1020-5.

60. Battisti, V., et al., Oxidative stress and antioxidant status in prostate cancer patients: relation to Gleason score, treatment and bone metastasis. Biomed Pharmacother, 2011. 65(7): p. 516-24.

61. Ng, C.F., et al., The rate of cellular hydrogen peroxide removal shows dependency on GSH: mathematical insight into in vivo $\mathrm{H} 2 \mathrm{O} 2$ and GPx concentrations. Free Radic Res, 2007. 41(11): p. 1201-11.

62. Lu, J. and A. Holmgren, The thioredoxin antioxidant system. Free Radic Biol Med, 2014. 66: p. 75-87.

63. Iynem, A.H., et al., The effect of prostate cancer and antiandrogenic therapy on lipid peroxidation and antioxidant systems. Int Urol Nephrol, 2004. 36(1): p. 57-62.

64. Srivastava, D.S. and R.D. Mittal, Free radical injury and antioxidant status in patients with benign prostate hyperplasia and prostate cancer. Indian J Clin Biochem, 2005. 20(2): p. 162-5.

65. Sikka, S.C., Role of oxidative stress response elements and antioxidants in prostate cancer pathobiology and chemoprevention--a mechanistic approach. Curr Med Chem, 2003. 10(24): p. 2679-92.

66. Sies, H., Hydrogen peroxide as a central redox signaling molecule in physiological oxidative stress: Oxidative eustress. Redox Biol, 2017. 11: p. 613-619. 
67. Sies, $\mathrm{H}$., Role of metabolic H2O2 generation: redox signaling and oxidative stress. J Biol Chem, 2014. 289(13): p. 8735-41.

68. Go, Y.M., J.D. Chandler, and D.P. Jones, The cysteine proteome. Free Radic Biol Med, 2015. 84: p. 227-245.

69. Gielis, J.F., et al., Oxidative and nitrosative stress during pulmonary ischemiareperfusion injury: from the lab to the OR. Ann Transl Med, 2017. 5(6): p. 131.

70. Bienert, G.P. and F. Chaumont, Aquaporin-facilitated transmembrane diffusion of hydrogen peroxide. Biochim Biophys Acta, 2014. 1840(5): p. 1596-604.

71. Reczek, C.R. and N.S. Chandel, ROS-dependent signal transduction. Curr Opin Cell Biol, 2015. 33: p. 8-13.

72. Netto, L.E. and F. Antunes, The Roles of Peroxiredoxin and Thioredoxin in Hydrogen Peroxide Sensing and in Signal Transduction. Mol Cells, 2016. 39(1): p. 65-71.

73. Hanschmann, E.M., et al., Thioredoxins, glutaredoxins, and peroxiredoxins-molecular mechanisms and health significance: from cofactors to antioxidants to redox signaling. Antioxid Redox Signal, 2013. 19(13): p. 1539-605.

74. Soukhova-O'Hare, G.K., et al., Postnatal intermittent hypoxia and developmental programming of hypertension in spontaneously hypertensive rats: the role of reactive oxygen species and L-Ca2+ channels. Hypertension, 2008. 52(1): p. 15662.

75. Yan, G.P., D. Bischa, and S.E. Bottle, Synthesis and properties of novel porphyrin spin probes containing isoindoline nitroxides. Free Radic Biol Med, 2007. 43(1): p. 111-6.

76. Lin, Y.T., et al., Manganous ion supplementation accelerates wild type development, enhances stress resistance, and rescues the life span of a shortlived Caenorhabditis elegans mutant. Free Radic Biol Med, 2006. 40(7): p. 118593.

77. Sessler, J.L. and R.A. Miller, Texaphyrins: new drugs with diverse clinical applications in radiation and photodynamic therapy. Biochem Pharmacol, 2000. 59(7): p. 733-9.

78. Chen, X., et al., Repair effects of exogenous SOD on Bacillus subtilis against gamma radiation exposure. J Environ Radioact, 2013. 126: p. 259-63.

79. Pan, J., et al., GST-TAT-SOD: Cell Permeable Bifunctional Antioxidant Enzyme-A Potential Selective Radioprotector. Oxid Med Cell Longev, 2016. 2016: p. 5935080.

80. Batinic-Haberle, I., et al., Design of Mn porphyrins for treating oxidative stress injuries and their redox-based regulation of cellular transcriptional activities. Amino Acids, 2012. 42(1): p. 95-113.

81. Batinic-Haberle, I., A. Tovmasyan, and I. Spasojevic, Mn Porphyrin-Based RedoxActive Drugs: Differential Effects as Cancer Therapeutics and Protectors of Normal Tissue Against Oxidative Injury. Antioxid Redox Signal, 2018. 29(16): p. 16911724.

82. Batinic-Haberle, I., et al., Diverse functions of cationic Mn(III) N-substituted pyridylporphyrins, recognized as SOD mimics. Free Radic Biol Med, 2011. 51(5): p. 1035-53.

83. Batinic-Haberle, I., A. Tovmasyan, and I. Spasojevic, An educational overview of the chemistry, biochemistry and therapeutic aspects of Mn porphyrins--From superoxide dismutation to H2O2-driven pathways. Redox Biol, 2015. 5: p. 43-65.

84. Batinic-Haberle, I., J.S. Reboucas, and I. Spasojevic, Superoxide dismutase mimics: chemistry, pharmacology, and therapeutic potential. Antioxid Redox Signal, 2010. 13(6): p. 877-918. 
85. Miriyala, S., et al., Manganese superoxide dismutase, MnSOD and its mimics. Biochim Biophys Acta, 2012. 1822(5): p. 794-814.

86. Ferrer-Sueta, G., et al., Reactions of manganese porphyrins and manganesesuperoxide dismutase with peroxynitrite. Methods Enzymol, 2002. 349: p. 23-37.

87. Spasojevic, I., et al., Mn porphyrin-based superoxide dismutase (SOD) mimic, MnIIITE-2-PyP5+, targets mouse heart mitochondria. Free Radic Biol Med, 2007. 42(8): p. 1193-200.

88. Kos, I., et al., Lipophilicity of potent porphyrin-based antioxidants: comparison of ortho and meta isomers of Mn(III) N-alkylpyridylporphyrins. Free Radic Biol Med, 2009. 47(1): p. 72-8.

89. Rabbani, Z.N., et al., Antiangiogenic action of redox-modulating Mn(III) mesotetrakis(N-ethylpyridinium-2-yl)porphyrin, MnTE-2-PyP(5+), via suppression of oxidative stress in a mouse model of breast tumor. Free Radic Biol Med, 2009. 47(7): p. 992-1004.

90. Tovmasyan, A., et al., Anticancer therapeutic potential of Mn porphyrin/ascorbate system. Free Radic Biol Med, 2015. 89: p. 1231-47.

91. Ye, X., et al., Cytotoxic effects of Mn(III) N-alkylpyridylporphyrins in the presence of cellular reductant, ascorbate. Free Radic Res, 2011. 45(11-12): p. 1289-306.

92. Weitzel, D.H., et al., Neurobehavioral radiation mitigation to standard brain cancer therapy regimens by $\mathrm{Mn}$ (III) n-butoxyethylpyridylporphyrin-based redox modifier. Environ Mol Mutagen, 2016. 57(5): p. 372-81.

93. Li, H., et al., Mn(III) meso-tetrakis-(N-ethylpyridinium-2-yl) porphyrin mitigates total body irradiation-induced long-term bone marrow suppression. Free Radic Biol Med, 2011. 51(1): p. 30-7.

94. Gauter-Fleckenstein, B., et al., Robust rat pulmonary radioprotection by a lipophilic Mn N-alkylpyridylporphyrin, MnTnHex-2-PyP(5+). Redox Biol, 2014. 2: p. 400-10.

95. Chatterjee, A., et al., The Addition of Manganese Porphyrins during Radiation Inhibits Prostate Cancer Growth and Simultaneously Protects Normal Prostate Tissue from Radiation Damage. Antioxidants (Basel), 2018. 7(1).

96. Chatterjee, A., E.A. Kosmacek, and R.E. Oberley-Deegan, MnTE-2-PyP Treatment, or NOX4 Inhibition, Protects against Radiation-Induced Damage in Mouse Primary Prostate Fibroblasts by Inhibiting the TGF-Beta 1 Signaling Pathway. Radiat Res, 2017. 187(3): p. 367-381.

97. Shrishrimal, S., et al., The SOD Mimic, MnTE-2-PyP, Protects from Chronic Fibrosis and Inflammation in Irradiated Normal Pelvic Tissues. Antioxidants (Basel), 2017. 6(4).

98. Jaramillo, M.C., et al., Manganese (III) meso-tetrakis N-ethylpyridinium-2-yl porphyrin acts as a pro-oxidant to inhibit electron transport chain proteins, modulate bioenergetics, and enhance the response to chemotherapy in lymphoma cells. Free Radic Biol Med, 2015. 83: p. 89-100.

99. Makinde, A.Y., et al., Effect of a metalloporphyrin antioxidant (MnTE-2-PyP) on the response of a mouse prostate cancer model to radiation. Anticancer Res, 2009. 29(1): p. 107-18.

100. Mehrotra, S., et al., Analysis of a metalloporphyrin antioxidant mimetic (MnTE-2$P y P)$ as a radiomitigator: prostate tumor and immune status. Technol Cancer Res Treat, 2012. 11(5): p. 447-57.

101. Cutruzzola, F., et al., Glucose Metabolism in the Progression of Prostate Cancer. Front Physiol, 2017. 8: p. 97.

102. Costello, L.C. and R.B. Franklin, The intermediary metabolism of the prostate: a key to understanding the pathogenesis and progression of prostate malignancy. Oncology, 2000. 59(4): p. 269-82. 
103. Tsui, K.H., P.L. Chang, and H.H. Juang, Zinc blocks gene expression of mitochondrial aconitase in human prostatic carcinoma cells. Int J Cancer, 2006. 118(3): p. 609-15.

104. Parlo, R.A. and P.S. Coleman, Enhanced rate of citrate export from cholesterolrich hepatoma mitochondria. The truncated Krebs cycle and other metabolic ramifications of mitochondrial membrane cholesterol. J Biol Chem, 1984. 259(16): p. 9997-10003.

105. Hammoudi, N., et al., Metabolic alterations in cancer cells and therapeutic implications. Chin J Cancer, 2011. 30(8): p. 508-25.

106. Cooper, J.F. and H. Imfeld, The role of citric acid in the physiology of the prostate: a preliminary report. J Urol, 1959. 81(1): p. 157-64.

107. Costello, L.C. and R.B. Franklin, The clinical relevance of the metabolism of prostate cancer; zinc and tumor suppression: connecting the dots. Mol Cancer, 2006. 5: p. 17.

108. Eidelman, E., et al., The Metabolic Phenotype of Prostate Cancer. Front Oncol, 2017. 7: p. 131.

109. Tennakoon, J.B., et al., Androgens regulate prostate cancer cell growth via an AMPK-PGC-1alpha-mediated metabolic switch. Oncogene, 2014. 33(45): p. 525161.

110. Shi, F., et al., Molecular properties, functions, and potential applications of NAD kinases. Acta Biochim Biophys Sin (Shanghai), 2009. 41(5): p. 352-61.

111. Xiao, W., et al., $N A D(H)$ and $N A D P(H)$ Redox Couples and Cellular Energy Metabolism. Antioxid Redox Signal, 2018. 28(3): p. 251-272.

112. Yang, Y. and A.A. Sauve, $N A D(+)$ metabolism: Bioenergetics, signaling and manipulation for therapy. Biochim Biophys Acta, 2016. 1864(12): p. 1787-1800.

113. Alano, C.C., et al., Differences among cell types in $N A D(+)$ compartmentalization: a comparison of neurons, astrocytes, and cardiac myocytes. J Neurosci Res, 2007. 85(15): p. 3378-85.

114. Di Lisa, F., et al., Opening of the mitochondrial permeability transition pore causes depletion of mitochondrial and cytosolic NAD+ and is a causative event in the death of myocytes in postischemic reperfusion of the heart. J Biol Chem, 2001. 276(4): p. 2571-5.

115. Sauve, A.A., NAD+ and vitamin B3: from metabolism to therapies. J Pharmacol Exp Ther, 2008. 324(3): p. 883-93.

116. Williamson, D.H., P. Lund, and H.A. Krebs, The redox state of free nicotinamideadenine dinucleotide in the cytoplasm and mitochondria of rat liver. Biochem $\mathrm{J}$, 1967. 103(2): p. 514-27.

117. Canto, C., K.J. Menzies, and J. Auwerx, NAD(+) Metabolism and the Control of Energy Homeostasis: A Balancing Act between Mitochondria and the Nucleus. Cell Metab, 2015. 22(1): p. 31-53.

118. Chmielewski, J.P., et al., CD38 Inhibits Prostate Cancer Metabolism and Proliferation by Reducing Cellular NAD(+) Pools. Mol Cancer Res, 2018. 16(11): p. 1687-1700.

119. Li, R., Y. Quan, and W. Xia, SIRT3 inhibits prostate cancer metastasis through regulation of FOXO3A by suppressing Wnt/beta-catenin pathway. Exp Cell Res, 2018. 364(2): p. 143-151.

120. Lee, J.H., et al., Identifying Dysregulated Epigenetic Enzyme Activity in CastrateResistant Prostate Cancer Development. ACS Chem Biol, 2017. 12(11): p. 28042814.

121. Alves-Fernandes, D.K. and M.G. Jasiulionis, The Role of SIRT1 on DNA Damage Response and Epigenetic Alterations in Cancer. Int J Mol Sci, 2019. 20(13). 
122. Wang, B., et al., NAMPT overexpression in prostate cancer and its contribution to tumor cell survival and stress response. Oncogene, 2011. 30(8): p. 907-21.

123. Sampath, D., et al., Inhibition of nicotinamide phosphoribosyltransferase (NAMPT) as a therapeutic strategy in cancer. Pharmacol Ther, 2015. 151: p. 16-31.

124. Zerp, S.F., et al., $N A D(+)$ depletion by APO866 in combination with radiation in a prostate cancer model, results from an in vitro and in vivo study. Radiother Oncol, 2014. 110(2): p. 348-54.

125. Blacker, T.S. and M.R. Duchen, Investigating mitochondrial redox state using NADH and NADPH autofluorescence. Free Radic Biol Med, 2016. 100: p. 53-65.

126. Goodman, R.P., S.E. Calvo, and V.K. Mootha, Spatiotemporal compartmentalization of hepatic NADH and NADPH metabolism. J Biol Chem, 2018. 293(20): p. 7508-7516.

127. Ren, J.G., et al., Induction of erythroid differentiation in human erythroleukemia cells by depletion of malic enzyme 2. PLoS One, 2010. 5(9).

128. Rydstrom, J., Mitochondrial NADPH, transhydrogenase and disease. Biochim Biophys Acta, 2006. 1757(5-6): p. 721-6.

129. Lunt, S.Y. and M.G. Vander Heiden, Aerobic glycolysis: meeting the metabolic requirements of cell proliferation. Annu Rev Cell Dev Biol, 2011. 27: p. 441-64.

130. Liu, L., et al., Malic enzyme tracers reveal hypoxia-induced switch in adipocyte NADPH pathway usage. Nat Chem Biol, 2016. 12(5): p. 345-52.

131. Lim, S.D., et al., Increased Nox1 and hydrogen peroxide in prostate cancer. Prostate, 2005. 62(2): p. 200-7.

132. Brar, S.S., et al., NOX5 NAD(P)H oxidase regulates growth and apoptosis in DU 145 prostate cancer cells. Am J Physiol Cell Physiol, 2003. 285(2): p. C353-69.

133. Arbiser, J.L., et al., Reactive oxygen generated by Nox1 triggers the angiogenic switch. Proc Natl Acad Sci U S A, 2002. 99(2): p. 715-20.

134. Agledal, L., M. Niere, and M. Ziegler, The phosphate makes a difference: cellular functions of NADP. Redox Rep, 2010. 15(1): p. 2-10.

135. Xu, J.Z., H.K. Yang, and W.G. Zhang, NADPH metabolism: a survey of its theoretical characteristics and manipulation strategies in amino acid biosynthesis. Crit Rev Biotechnol, 2018. 38(7): p. 1061-1076.

136. Qu, Q., et al., Fatty acid oxidation and carnitine palmitoyltransferase l: emerging therapeutic targets in cancer. Cell Death Dis, 2016. 7: p. e2226.

137. Goto, T., et al., The expression profile of phosphatidylinositol in high spatial resolution imaging mass spectrometry as a potential biomarker for prostate cancer. PLoS One, 2014. 9(2): p. e90242.

138. Louie, S.M., et al., Cancer cells incorporate and remodel exogenous palmitate into structural and oncogenic signaling lipids. Biochim Biophys Acta, 2013. 1831(10): p. 1566-72.

139. Fan, J., et al., Quantitative flux analysis reveals folate-dependent NADPH production. Nature, 2014. 510(7504): p. 298-302.

140. Ducker, G.S. and J.D. Rabinowitz, One-Carbon Metabolism in Health and Disease. Cell Metab, 2017. 25(1): p. 27-42.

141. Vettore, L., R.L. Westbrook, and D.A. Tennant, New aspects of amino acid metabolism in cancer. Br J Cancer, 2020. 122(2): p. 150-156.

142. Tong, Q., et al., MnTE-2-PyP modulates thiol oxidation in a hydrogen peroxidemediated manner in a human prostate cancer cell. Free Radic Biol Med, 2016. 101: p. 32-43.

143. Behring, J.B., et al., Does reversible cysteine oxidation link the Western diet to cardiac dysfunction? FASEB J, 2014. 28(5): p. 1975-87. 
144. Swingle, M., L. Ni, and R.E. Honkanen, Small-molecule inhibitors of ser/thr protein phosphatases: specificity, use and common forms of abuse. Methods Mol Biol, 2007. 365: p. 23-38.

145. Yang, N.C., et al., A convenient one-step extraction of cellular ATP using boiling water for the luciferin-Iuciferase assay of ATP. Anal Biochem, 2002. 306(2): p. 3237.

146. Gunda, V., F. Yu, and P.K. Singh, Validation of Metabolic Alterations in Microscale Cell Culture Lysates Using Hydrophilic Interaction Liquid Chromatography (HILIC)Tandem Mass Spectrometry-Based Metabolomics. PLoS One, 2016. 11(4): p. e0154416.

147. Berg, K., et al., A high precision method for quantitative measurements of reactive oxygen species in frozen biopsies. PLoS One, 2014. 9(3): p. e90964.

148. Nazarewicz, R.R., A. Bikineyeva, and S.I. Dikalov, Rapid and specific measurements of superoxide using fluorescence spectroscopy. J Biomol Screen, 2013. 18(4): p. 498-503.

149. Arnold, R.S., et al., Hydrogen peroxide mediates the cell growth and transformation caused by the mitogenic oxidase Nox1. Proc Natl Acad Sci U S A, 2001. 98(10): p. 5550-5.

150. Preston, T.J., W.J. Muller, and G. Singh, Scavenging of extracellular H2O2 by catalase inhibits the proliferation of HER-2/Neu-transformed rat-1 fibroblasts through the induction of a stress response. J Biol Chem, 2001. 276(12): p. 955864.

151. Smit-de Vries, M.P., et al., Resistance of quiescent and proliferating airway epithelial cells to H2O2 challenge. Eur Respir J, 2007. 29(4): p. 633-42.

152. Santos, C.X., et al., Targeted redox inhibition of protein phosphatase 1 by Nox 4 regulates elF2alpha-mediated stress signaling. EMBO J, 2016. 35(3): p. 319-34.

153. Zhang, H., et al., Theoretical studies on the reaction mechanism of PP1 and the effects of different oxidation states of the Mn-Mn center on the mechanism. J Biol Inorg Chem, 2013. 18(4): p. 451-9.

154. Virshup, D.M. and S. Shenolikar, From promiscuity to precision: protein phosphatases get a makeover. Mol Cell, 2009. 33(5): p. 537-45.

155. Kolupaeva, V. and V. Janssens, PP1 and PP2A phosphatases--cooperating partners in modulating retinoblastoma protein activation. FEBS J, 2013. 280(2): p. 627-43.

156. Cohen, P.T., Protein phosphatase 1--targeted in many directions. J Cell Sci, 2002. 115(Pt 2): p. 241-56.

157. Kim, H.S., G. Fernandes, and C.W. Lee, Protein Phosphatases Involved in Regulating Mitosis: Facts and Hypotheses. Mol Cells, 2016. 39(9): p. 654-62.

158. Hsu, L.C., et al., Gene amplification and overexpression of protein phosphatase 1 alpha in oral squamous cell carcinoma cell lines. Oncogene, 2006. 25(40): p. 5517-26.

159. Edelson, J.R. and D.L. Brautigan, The Discodermia calyx toxin calyculin a enhances cyclin D1 phosphorylation and degradation, and arrests cell cycle progression in human breast cancer cells. Toxins (Basel), 2011. 3(1): p. 105-19.

160. Cicchillitti, L., et al., Oxidative stress induces protein phosphatase 2A-dependent dephosphorylation of the pocket proteins pRb, p107, and p130. J Biol Chem, 2003. 278(21): p. 19509-17.

161. Junttila, M.R., et al., CIP2A inhibits PP2A in human malignancies. Cell, 2007. 130(1): p. 51-62.

162. Remmerie, M. and V. Janssens, PP2A: A Promising Biomarker and Therapeutic Target in Endometrial Cancer. Front Oncol, 2019. 9: p. 462. 
163. Alt, J.R., et al., Phosphorylation-dependent regulation of cyclin D1 nuclear export and cyclin D1-dependent cellular transformation. Genes Dev, 2000. 14(24): p. 3102-14.

164. Guo, Y., et al., Phosphorylation of cyclin D1 at Thr 286 during S phase leads to its proteasomal degradation and allows efficient DNA synthesis. Oncogene, 2005. 24(16): p. 2599-612.

165. Kusume, T., et al., The $p 16$-cyclin D1/CDK4-pRb pathway and clinical outcome in epithelial ovarian cancer. Clin Cancer Res, 1999. 5(12): p. 4152-7.

166. Dai, M., et al., Cyclin D1 cooperates with p21 to regulate TGFbeta-mediated breast cancer cell migration and tumor local invasion. Breast Cancer Res, 2013. 15(3): p. R49.

167. Stein, G.H., et al., Differential roles for cyclin-dependent kinase inhibitors $p 21$ and $p 16$ in the mechanisms of senescence and differentiation in human fibroblasts. Mol Cell Biol, 1999. 19(3): p. 2109-17.

168. Roche, B., B. Arcangioli, and R. Martienssen, Transcriptional reprogramming in cellular quiescence. RNA Biol, 2017. 14(7): p. 843-853.

169. Jaramillo, M.C., et al., Manganese porphyrin, MnTE-2-PyP5+, Acts as a prooxidant to potentiate glucocorticoid-induced apoptosis in lymphoma cells. Free Radic Biol Med, 2012. 52(8): p. 1272-84.

170. Gupta, V. and R.N. Bamezai, Human pyruvate kinase M2: a multifunctional protein. Protein Sci, 2010. 19(11): p. 2031-44.

171. Anastasiou, D., et al., Inhibition of pyruvate kinase $M 2$ by reactive oxygen species contributes to cellular antioxidant responses. Science, 2011. 334(6060): p. 127883.

172. Lambeth, J.D., NOX enzymes and the biology of reactive oxygen. Nat Rev Immunol, 2004. 4(3): p. 181-9.

173. Oberley-Deegan, R.E., et al., The antioxidant, MnTE-2-PyP, prevents side-effects incurred by prostate cancer irradiation. PLoS One, 2012. 7(9): p. e44178.

174. Batinic-Haberle, I., et al., Pure MnTBAP selectively scavenges peroxynitrite over superoxide: comparison of pure and commercial MnTBAP samples to MnTE-2PyP in two models of oxidative stress injury, an SOD-specific Escherichia coli model and carrageenan-induced pleurisy. Free Radic Biol Med, 2009. 46(2): p. 192-201.

175. Shrishrimal, S., et al., Manganese porphyrin, MnTE-2-PyP, treatment protects the prostate from radiation-induced fibrosis (RIF) by activating the NRF2 signaling pathway and enhancing SOD2 and sirtuin activity. Free Radic Biol Med, 2020. 152: p. 255-270.

176. Wu, C.W., et al., Combined treatment with vitamin $C$ and methotrexate inhibits triple-negative breast cancer cell growth by increasing $\mathrm{H} 2 \mathrm{O} 2$ accumulation and activating caspase-3 and p38 pathways. Oncol Rep, 2017. 37(4): p. 2177-2184.

177. Freitas, M., et al., Oxidative stress adaptation in aggressive prostate cancer may be counteracted by the reduction of glutathione reductase. FEBS Open Bio, 2012. 2: p. 119-28.

178. Bae, J.Y., et al., Peroxiredoxin I and II inhibit H2O2-induced cell death in MCF-7 cell lines. J Cell Biochem, 2007. 101(4): p. 1038-45.

179. Deshpande, N.N., et al., Mechanism of hydrogen peroxide-induced cell cycle arrest in vascular smooth muscle. Antioxid Redox Signal, 2002. 4(5): p. 845-54.

180. Wegman-Points, L.J., et al., Retroviral-infection increases tumorigenic potential of MDA-MB-231 breast carcinoma cells by expanding an aldehyde dehydrogenase (ALDH1) positive stem-cell like population. Redox Biol, 2014. 2: p. 847-54. 
181. Hahm, E.R., K. Sakao, and S.V. Singh, Honokiol activates reactive oxygen species-mediated cytoprotective autophagy in human prostate cancer cells. Prostate, 2014. 74(12): p. 1209-21.

182. Hyoudou, K., et al., Inhibition of metastatic tumor growth in mouse lung by repeated administration of polyethylene glycol-conjugated catalase: quantitative analysis with firefly luciferase-expressing melanoma cells. Clin Cancer Res, 2004. 10(22): p. 7685-91.

183. Zmijewski, J.W., et al., Exposure to hydrogen peroxide induces oxidation and activation of AMP-activated protein kinase. J Biol Chem, 2010. 285(43): p. 3315464.

184. Veal, E.A., A.M. Day, and B.A. Morgan, Hydrogen peroxide sensing and signaling. Mol Cell, 2007. 26(1): p. 1-14.

185. McStay, G.P., S.J. Clarke, and A.P. Halestrap, Role of critical thiol groups on the matrix surface of the adenine nucleotide translocase in the mechanism of the mitochondrial permeability transition pore. Biochem J, 2002. 367(Pt 2): p. 541-8.

186. Mitchell, A.R., et al., Redox regulation of pyruvate kinase M2 by cysteine oxidation and S-nitrosation. Biochem J, 2018. 475(20): p. 3275-3291.

187. Perry, S.W., et al., Mitochondrial membrane potential probes and the proton gradient: a practical usage guide. Biotechniques, 2011. 50(2): p. 98-115.

188. Ademowo, O.S., et al., Lipid (per) oxidation in mitochondria: an emerging target in the ageing process? Biogerontology, 2017. 18(6): p. 859-879.

189. Anderson, E.J., L.A. Katunga, and M.S. Willis, Mitochondria as a source and target of lipid peroxidation products in healthy and diseased heart. Clin Exp Pharmacol Physiol, 2012. 39(2): p. 179-93.

190. Cowen, D., et al., Ki-67 staining is an independent correlate of biochemical failure in prostate cancer treated with radiotherapy. Clin Cancer Res, 2002. 8(5): p. 114854.

191. Li, H., et al., Ki67 as a predictor of poor prognosis in patients with triple-negative breast cancer. Oncol Lett, 2015. 9(1): p. 149-152.

192. Gonzalez-Magana, A. and F.J. Blanco, Human PCNA Structure, Function and Interactions. Biomolecules, 2020. 10(4).

193. Bologna-Molina, R., et al., Comparison of the value of PCNA and Ki-67 as markers of cell proliferation in ameloblastic tumors. Med Oral Patol Oral Cir Bucal, 2013. 18(2): p. e174-9.

194. Thakar, T., et al., Ubiquitinated-PCNA protects replication forks from DNA2mediated degradation by regulating Okazaki fragment maturation and chromatin assembly. Nat Commun, 2020. 11(1): p. 2147.

195. Shivji, K.K., M.K. Kenny, and R.D. Wood, Proliferating cell nuclear antigen is required for DNA excision repair. Cell, 1992. 69(2): p. 367-74.

196. Wang, Y.P., et al., Regulation of G6PD acetylation by SIRT2 and KAT9 modulates NADPH homeostasis and cell survival during oxidative stress. EMBO J, 2014. 33(12): p. 1304-20.

197. Xu, S.N., et al., SIRT2 activates G6PD to enhance NADPH production and promote leukaemia cell proliferation. Sci Rep, 2016. 6: p. 32734.

198. Liu, R., et al., Tyrosine phosphorylation activates 6-phosphogluconate dehydrogenase and promotes tumor growth and radiation resistance. Nat Commun, 2019. 10(1): p. 991.

199. Shan, C., et al., Lysine acetylation activates 6-phosphogluconate dehydrogenase to promote tumor growth. Mol Cell, 2014. 55(4): p. 552-65. 
200. Kachadourian, R., et al., Synthesis and in vitro antioxidant properties of manganese(III) beta-octabromo-meso-tetrakis(4-carboxyphenyl)porphyrin. J Inorg Biochem, 2003. 95(4): p. 240-8.

201. Sheng, $\mathrm{H}_{\text {., }}$ et al., Mouse spinal cord compression injury is ameliorated by intrathecal cationic manganese(III) porphyrin catalytic antioxidant therapy. Neurosci Lett, 2004. 366(2): p. 220-5.

202. Vujaskovic, Z., et al., A small molecular weight catalytic metalloporphyrin antioxidant with superoxide dismutase (SOD) mimetic properties protects lungs from radiation-induced injury. Free Radic Biol Med, 2002. 33(6): p. 857-63.

203. Batinic-Haberle, I., Z. Rajic, and L. Benov, A combination of two antioxidants (an SOD mimic and ascorbate) produces a pro-oxidative effect forcing Escherichia coli to adapt via induction of oxyR regulon. Anticancer Agents Med Chem, 2011. 11(4): p. 329-40.

204. Ferrer-Sueta, G., et al., Reduction of manganese porphyrins by flavoenzymes and submitochondrial particles: a catalytic cycle for the reduction of peroxynitrite. Free Radic Biol Med, 2006. 41(3): p. 503-12.

205. Carballal, S., et al., Manganese porphyrin redox state in endothelial cells: Resonance Raman studies and implications for antioxidant protection towards peroxynitrite. Free Radic Biol Med, 2018. 126: p. 379-392.

206. Kachadourian, R., et al., Flavin-dependent antioxidant properties of a new series of meso-N,N'-dialkyl-imidazolium substituted manganese(III) porphyrins. Biochem Pharmacol, 2004. 67(1): p. 77-85.

207. Faulkner, K.M., S.I. Liochev, and I. Fridovich, Stable Mn(III) porphyrins mimic superoxide dismutase in vitro and substitute for it in vivo. J Biol Chem, 1994. 269(38): p. 23471-6.

208. Tovmasyan, A., et al., Design, mechanism of action, bioavailability and therapeutic effects of mn porphyrin-based redox modulators. Med Princ Pract, 2013. 22(2): p. 103-30.

209. Vasdev, S., V.D. Gill, and P.K. Singal, Modulation of oxidative stress-induced changes in hypertension and atherosclerosis by antioxidants. Exp Clin Cardiol, 2006. 11(3): p. 206-16.

210. Treberg, J.R. and M.D. Brand, A model of the proton translocation mechanism of complex I. J Biol Chem, 2011. 286(20): p. 17579-84.

211. Batinic-Haberle, I. and M.E. Tome, Thiol regulation by Mn porphyrins, commonly known as SOD mimics. Redox Biol, 2019. 25: p. 101139.

212. Buckel, W. and R.K. Thauer, Energy conservation via electron bifurcating ferredoxin reduction and proton/Na(+) translocating ferredoxin oxidation. Biochim Biophys Acta, 2013. 1827(2): p. 94-113.

213. Rutter, J., D.R. Winge, and J.D. Schiffman, Succinate dehydrogenase - Assembly, regulation and role in human disease. Mitochondrion, 2010. 10(4): p. 393-401.

214. Ferrer-Sueta, G., et al., Catalytic scavenging of peroxynitrite by isomeric Mn(III) Nmethylpyridylporphyrins in the presence of reductants. Chem Res Toxicol, 1999. 12(5): p. 442-9.

215. Shimizu, M., et al., Mechanism of de novo branched-chain amino acid synthesis as an alternative electron sink in hypoxic Aspergillus nidulans cells. Appl Environ Microbiol, 2010. 76(5): p. 1507-15.

216. Ross, D. and D. Siegel, Functions of NQO1 in Cellular Protection and CoQ10 Metabolism and its Potential Role as a Redox Sensitive Molecular Switch. Front Physiol, 2017. 8: p. 595. 
217. Teramoto, S., et al., Hydrogen peroxide-induced apoptosis and necrosis in human lung fibroblasts: protective roles of glutathione. Jpn J Pharmacol, 1999. 79(1): p. 33-40.

218. Saito, Y., et al., Turning point in apoptosis/necrosis induced by hydrogen peroxide. Free Radic Res, 2006. 40(6): p. 619-30.

219. Park, W.H., Hydrogen peroxide inhibits the growth of lung cancer cells via the induction of cell death and G1phase arrest. Oncol Rep, 2018. 40(3): p. 1787-1794.

220. Bas, E., M. Naziroglu, and L. Pecze, ADP-Ribose and oxidative stress activate TRPM8 channel in prostate cancer and kidney cells. Sci Rep, 2019. 9(1): p. 4100.

221. Dreher, D. and A.F. Junod, Role of oxygen free radicals in cancer development. Eur J Cancer, 1996. 32A(1): p. 30-8.

222. Chatterjee, A., et al., MnTE-2-PyP, a manganese porphyrin, reduces cytotoxicity caused by irradiation in a diabetic environment through the induction of endogenous antioxidant defenses. Redox Biol, 2020. 34: p. 101542.

223. Boss, M.K., et al., Potential for a novel manganese porphyrin compound as adjuvant canine lymphoma therapy. Cancer Chemother Pharmacol, 2017. 80(2): p. 421-431.

224. Bagnyukova, T.V., et al., Chemotherapy and signaling: How can targeted therapies supercharge cytotoxic agents? Cancer Biol Ther, 2010. 10(9): p. 839-53.

225. Yang, $\mathrm{H}_{\text {., }}$ et al., The role of cellular reactive oxygen species in cancer chemotherapy. J Exp Clin Cancer Res, 2018. 37(1): p. 266.

226. Mackler, N.J. and K.J. Pienta, Drug insight: Use of docetaxel in prostate and urothelial cancers. Nat Clin Pract Urol, 2005. 2(2): p. 92-100; quiz 1 p following 112.

227. Lyseng-Williamson, K.A. and C. Fenton, Docetaxel: a review of its use in metastatic breast cancer. Drugs, 2005. 65(17): p. 2513-31.

228. Haldar, S., J. Chintapalli, and C.M. Croce, Taxol induces bcl-2 phosphorylation and death of prostate cancer cells. Cancer Res, 1996. 56(6): p. 1253-5.

229. Sborov, D.W., B.M. Haverkos, and P.J. Harris, Investigational cancer drugs targeting cell metabolism in clinical development. Expert Opin Investig Drugs, 2015. 24(1): p. 79-94.

230. Stein, M., et al., Targeting tumor metabolism with 2-deoxyglucose in patients with castrate-resistant prostate cancer and advanced malignancies. Prostate, 2010. 70(13): p. 1388-94.

231. Anassi, E. and U.A. Ndefo, Sipuleucel-T (provenge) injection: the first immunotherapy agent (vaccine) for hormone-refractory prostate cancer. P T, 2011. 36(4): p. 197-202.

232. Antonarakis, E.S. and C.G. Drake, Current status of immunological therapies for prostate cancer. Curr Opin Urol, 2010. 20(3): p. 241-6.

233. Hodi, F.S., et al., Improved survival with ipilimumab in patients with metastatic melanoma. N Engl J Med, 2010. 363(8): p. 711-23.

234. Hannani, D., et al., Anticancer immunotherapy by CTLA-4 blockade: obligatory contribution of IL-2 receptors and negative prognostic impact of soluble CD25. Cell Res, 2015. 25(2): p. 208-24.

235. Okazaki, T., et al., PD-1 immunoreceptor inhibits $B$ cell receptor-mediated signaling by recruiting src homology 2-domain-containing tyrosine phosphatase 2 to phosphotyrosine. Proc Natl Acad Sci U S A, 2001. 98(24): p. 13866-71.

236. Fife, B.T., et al., Interactions between $P D-1$ and $P D-L 1$ promote tolerance by blocking the TCR-induced stop signal. Nat Immunol, 2009. 10(11): p. 1185-92. 
237. Hodi, F.S., et al., Nivolumab plus ipilimumab or nivolumab alone versus ipilimumab alone in advanced melanoma (CheckMate 067): 4-year outcomes of a multicentre, randomised, phase 3 trial. Lancet Oncol, 2018. 19(11): p. 1480-1492.

238. Kantoff, P.W., et al., Sipuleucel-T immunotherapy for castration-resistant prostate cancer. N Engl J Med, 2010. 363(5): p. 411-22.

239. Topalian, S.L., et al., Safety, activity, and immune correlates of anti-PD-1 antibody in cancer. N Engl J Med, 2012. 366(26): p. 2443-54.

240. Kwon, E.D., et al., Ipilimumab versus placebo after radiotherapy in patients with metastatic castration-resistant prostate cancer that had progressed after docetaxel chemotherapy (CA184-043): a multicentre, randomised, double-blind, phase 3 trial. Lancet Oncol, 2014. 15(7): p. 700-12.

241. Finkelstein, S.E., et al., Serial assessment of lymphocytes and apoptosis in the prostate during coordinated intraprostatic dendritic cell injection and radiotherapy. Immunotherapy, 2012. 4(4): p. 373-82.

242. Slovin, S.F., et al., Ipilimumab alone or in combination with radiotherapy in metastatic castration-resistant prostate cancer: results from an open-label, multicenter phase I/II study. Ann Oncol, 2013. 24(7): p. 1813-1821.

243. Finkelstein, S.E., et al., Combining immunotherapy and radiation for prostate cancer. Clin Genitourin Cancer, 2015. 13(1): p. 1-9.

244. Tang, C., et al., Combining radiation and immunotherapy: a new systemic therapy for solid tumors? Cancer Immunol Res, 2014. 2(9): p. 831-8.

245. $\mathrm{Li}, \mathrm{X}$., et al., Lessons learned from the blockade of immune checkpoints in cancer immunotherapy. J Hematol Oncol, 2018. 11(1): p. 31.

246. $\mathrm{Li}$, X., et al., Harnessing tumor-associated macrophages as aids for cancer immunotherapy. Mol Cancer, 2019. 18(1): p. 177.

247. Finkelstein, S.E. and M. Fishman, Clinical opportunities in combining immunotherapy with radiation therapy. Front Oncol, 2012. 2: p. 169.

248. Vanneman, M. and G. Dranoff, Combining immunotherapy and targeted therapies in cancer treatment. Nat Rev Cancer, 2012. 12(4): p. 237-51.

249. Johnke, R.M., et al., Response of T lymphocyte populations in prostate cancer patients undergoing radiotherapy: influence of neoajuvant total androgen suppression. Anticancer Res, 2005. 25(4): p. 3159-66.

250. Carvalho, H.A. and R.C. Villar, Radiotherapy and immune response: the systemic effects of a local treatment. Clinics (Sao Paulo), 2018. 73(suppl 1): p. e557s.

251. Oh, D.Y., et al., Immune Toxicities Elicted by CTLA-4 Blockade in Cancer Patients Are Associated with Early Diversification of the T-cell Repertoire. Cancer Res, 2017. 77(6): p. 1322-1330.

252. Jindal, V., Immunotherapy: a glimmer of hope for metastatic prostate cancer. Chin Clin Oncol, 2018. 7(6): p. 61.

253. Kesarwani, P., et al., Redox regulation of T-cell function: from molecular mechanisms to significance in human health and disease. Antioxid Redox Signal, 2013. 18(12): p. 1497-534.

254. Delmastro-Greenwood, M.M., et al., Mn porphyrin regulation of aerobic glycolysis: implications on the activation of diabetogenic immune cells. Antioxid Redox Signal, 2013. 19(16): p. 1902-15.

255. Raedler, L.A., Keytruda (Pembrolizumab): First PD-1 Inhibitor Approved for Previously Treated Unresectable or Metastatic Melanoma. Am Health Drug Benefits, 2015. 8(Spec Feature): p. 96-100.

256. Teng, F., et al., Radiotherapy combined with immune checkpoint blockade immunotherapy: Achievements and challenges. Cancer Lett, 2015. 365(1): p. 239. 
257. Badiyan, S.N., et al., Combining immunotherapy with radiation therapy in thoracic oncology. J Thorac Dis, 2018. 10(Suppl 21): p. S2492-S2507.

258. Cushman, T.R., et al., Combining radiation plus immunotherapy to improve systemic immune response. J Thorac Dis, 2018. 10(Suppl 3): p. S468-S479.

259. Harris, T.J., et al., Radiotherapy augments the immune response to prostate cancer in a time-dependent manner. Prostate, 2008. 68(12): p. 1319-29.

260. Xu, H.M., Th1 cytokine-based immunotherapy for cancer. Hepatobiliary Pancreat Dis Int, 2014. 13(5): p. 482-94.

261. Frossi, B., et al., Oxidative microenvironment exerts an opposite regulatory effect on cytokine production by Th1 and Th2 cells. Mol Immunol, 2008. 45(1): p. 58-64.

262. Kidd, P., Th1/Th2 balance: the hypothesis, its limitations, and implications for health and disease. Altern Med Rev, 2003. 8(3): p. 223-46.

263. Jungsuwadee, P., et al., The metalloporphyrin antioxidant, MnTE-2-PyP, inhibits Th2 cell immune responses in an asthma model. Int J Mol Sci, 2012. 13(8): p. 9785-97. 